\title{
Protein-selective adsorbers by molecular imprinting via a novel two-step surface grafting method
}

\author{
by \\ Dongxu Yin \\ Anhui, China
}

Thesis submitted to the Department of Chemistry of Universität Duisburg-Essen, in partial fulfillment of the requirements of the

$$
\begin{aligned}
& \text { degree of } \\
& \text { Dr. rer. nat. }
\end{aligned}
$$

Approved by the examining committee on October 16, 2013:

Chair : Prof. Dr. Bettina Siebers

Advisor : Prof. Dr. Mathias Ulbricht

Reviewer : Prof. Dr. Jochen S. Gutmann 


\section{Abstract}

Molecularly imprinted polymers (MIP) offer in principle a robust, cost-efficient alternative to antibodies, but it is still a challenge to develop such materials for protein recognition. Here we report the molecular imprinting of a functional polymeric hydrogel layer with whole protein lysozyme as template in two-step grafting procedure by a novel initiation approach on track-etched (TE) polyethylene terephthalate (PET) and cellulose membrane surfaces.

This two-step grafting strategy is based on surface functionalization with aliphatic $\mathrm{C}-\mathrm{Br}$ groups which can be used as initiator for surface-initiated atom transfer radical polymerization (SI-ATRP) and photo-initiated copolymerization. At first, the scaffold poly(methacrylic acid) (PMAA) was obtained through SI-ATRP of poly(tert.-butyl methacrylate) (PtBMA) and subsequent hydrolysis. Thereafter, it was assembled with the template to form a stable PMAA/protein complex. In the second step, a polyacrylamide (PAAm) hydrogel was synthesized via surface UV-initiated grafting/crosslinking copolymerization around the scaffold/protein complex. Finally, the template was eluted to yield the grafted hydrogel layer with binding sites having complementary size, shape and appropriate arrangement of the functional groups to rebind the target protein. The selectivity of lysozyme recognition, relative to cytochrome $\mathrm{C}$ with similar size and isoelectric point, was increased by optimizations of scaffold chain length, UV grafting/crosslinking time and chemical crosslinking degree of the PAAm-based hydrogel on TE PET membrane. The feasibility for the development of protein MIP in a straightforward way by independent optimization of crucial parameters (structures of scaffold with functional groups and of crosslinked hydrogel matrix) has been demonstrated.

The novel strategy of two-step grafting was further tested to develop immunoglobulin G imprinting on PET membrane. Immunoglobulin $\mathrm{G}$ has much bigger size than that of lysozyme. Through optimizations of scaffold and crosslinking degree, a satisfactory property of immunoglobulin $\mathrm{G}$ imprinted membrane was obtained and evaluated via comparison of binding properties for immunoglobulin $G$ and human serum albumin. Mixture of immunoglobulin $G$ and human serum albumin is an important biological background for protein separation and production. Thereafter, cellulose membrane with much more specific surface area and higher porosity than PET membrane was chosen as the new substrate for 
lysozyme imprinting. The imprinted structure was successfully synthesized on cellulose membrane via improvement of the UV-irradiation intensity and monomer concentration by UV-grafting/crosslinking. The property of the lysozyme imprinted cellulose membrane was improved via the similar optimization what has done during imprinting lysozyme on PET membrane.

In order to improve the control of imprinted polymer structure, living polymerization method reversible addition fragmentation chain transfer polymerization (RAFT) was introduced to combine with ATRP instead of the conventional UV-irradiation of C-Br groups. ATRP initiator benzyl chloride and UV-RAFT initiator dithiocarbamate were immobilized on PET membrane surface and successfully characterized via their reactivity in surface-initiated ATRP and RAFT, respectively. The independency of the initiation of these two initiators was achieved on the surface covered with $50 \%$ benzyl chloride and $50 \%$ dithiocarbamate, this showed a good potential to be applied for the protein imprinting. 
This work was performed during the period from January 2010 to January 2013 at the Institute of Technical Chemistry (Lehrstuhl für Technische Chemie II), Department of Chemistry, Universität Duisburg Essen, under the supervision of Prof. Mathias Ulbricht.

I declare that this dissertation represents my own work, except where due acknowledgement is made.

Dongxu Yin 


\section{Acknowledgments}

At first, I want to express my deepest gratitude to Prof. Dr. Mathias Ulbricht, who enables me to work at Technishe Chemie II group, Universität Duisburg-Essen. He gave me constructive guidance and offered active discussion with his profound knowledge and rich research experiences throughout my research work. Additionally, his kindness and patience to students impressed me a lot.

I am very grateful to reviewer Prof. Dr. Jochen S. Gutmann and chair Prof. Bettina Siebers for this thesis.

I would like to thank my students Qian (Gong), Christopher (Schartner) and Thorsten (van den Berg), who contributed on some experiments of this thesis.

I am very happy with the friendly working atmosphere in our research group at Lehrstuhl für Technische Chemie II of Universität Duisburg-Essen, special thanks go to Qian (Yang), Inge, Claudia, Haofei, Jun, Jing, Jürgen, Mathias, Polina, Eva, Falk, Anne, Oxana, Xi, Alele, Bintasan, Thomas, Jan, David, Robert, Dana, Thorsten (Pieper), Alexander, Sven (Frost), Sven (Behnke), Christian (Kaul), Christian (Kuhn), Christian (Erdmann), Alexandra (Wittmar), Alexandra (Gajda), Tobias (Hennecke), Tobias (Kallweit), Macal (Casas-Cartagena), Marcel (Gawenda), Nico, Miao, Jackelyn, Martyna, Hana, Kevin, Dominic, and Frau Nordmann.

I am grateful to Juncheng and Qianqian from organic chemistry group Schmuck of Universität Duisburg-Essen for their active discussion during my work. Moreover, I am pleased to thank Heath and Ranil for the support of XPS measurement.

Last but no least, I would love to pay my special gratitude to my grandparents, my parents, my wife, my brother and my whole family for their endless love, support and understanding. Specially, I would like express my best gratitude to my uncle Chunma of blessed memory, for his endless love and stimulation. 
This thesis is dedicated to

$$
\text { my parents and my wife }
$$

for their endless love and encouragement 


\section{Contents}

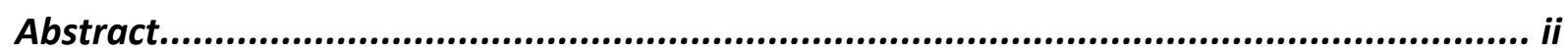

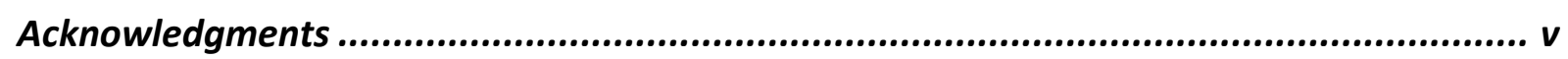

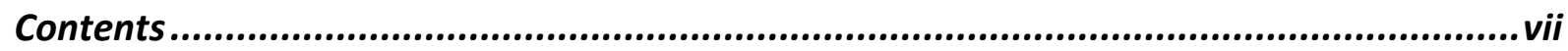

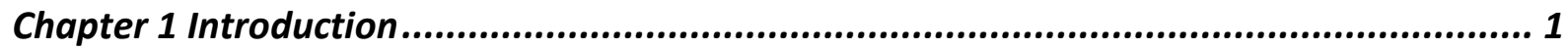

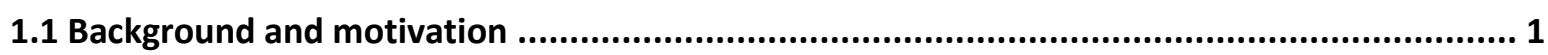

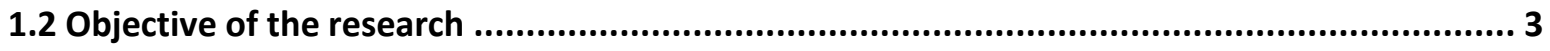

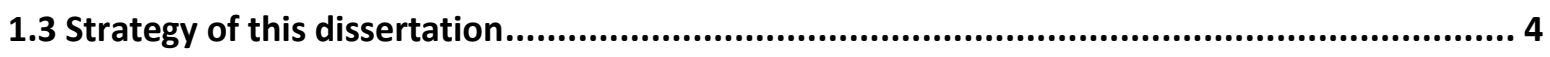

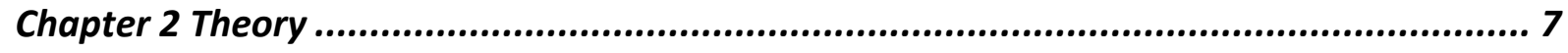

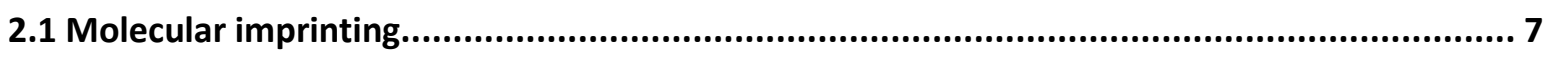

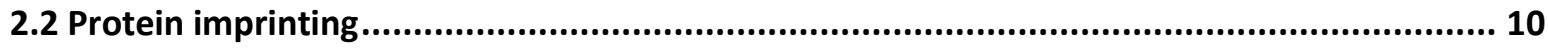

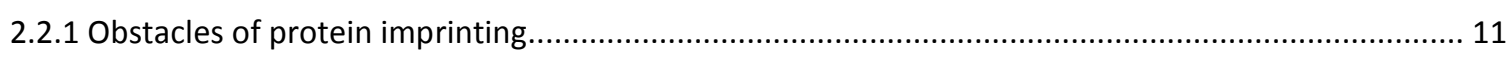

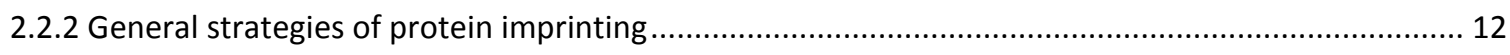

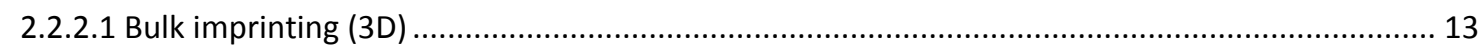

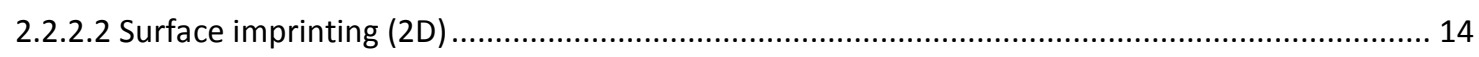

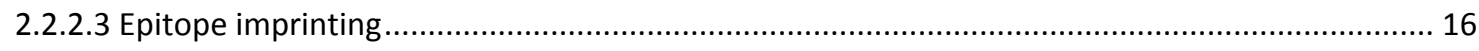

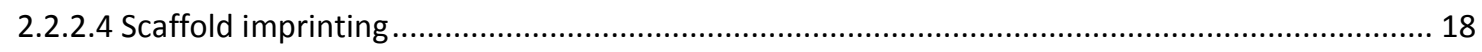

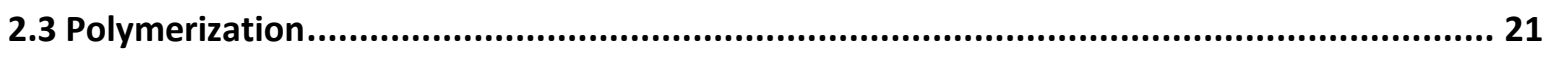

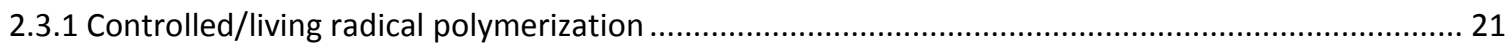

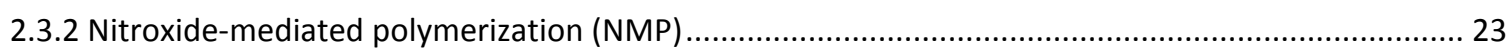

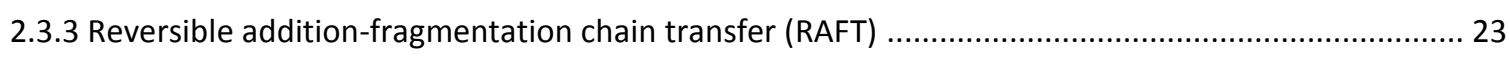

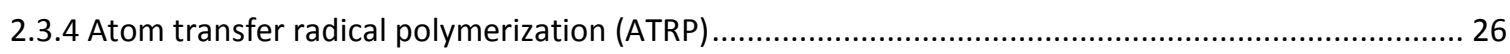

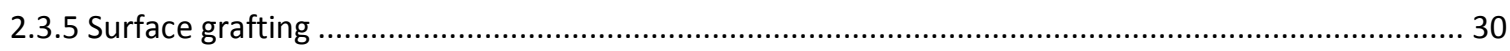

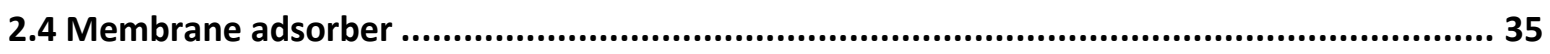

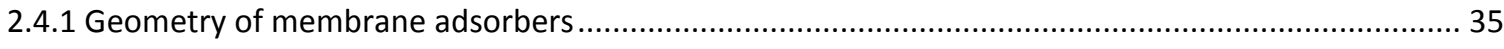

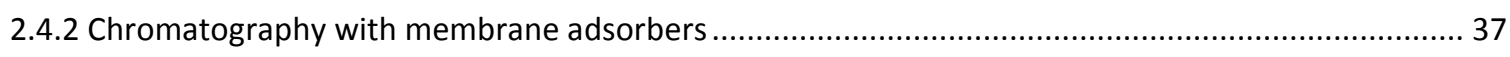

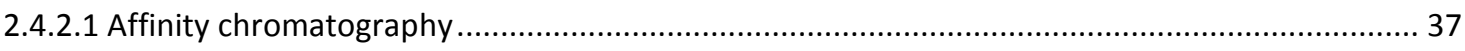

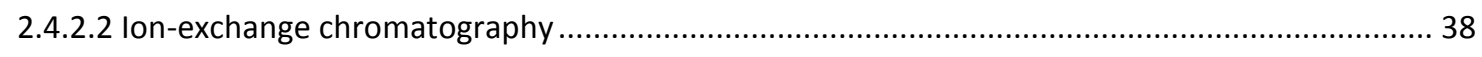

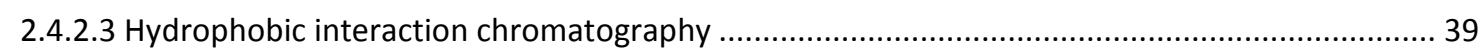

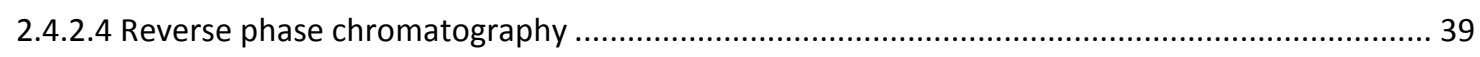

2.4.3 Molecular imprinted composite membrane adsorbers ........................................................ 40

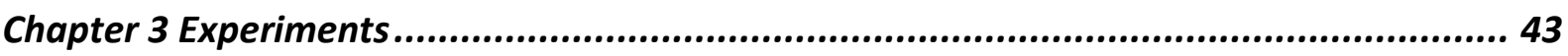

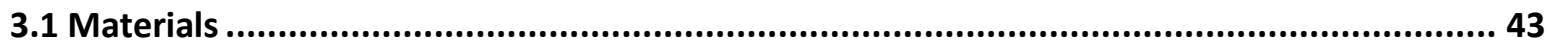

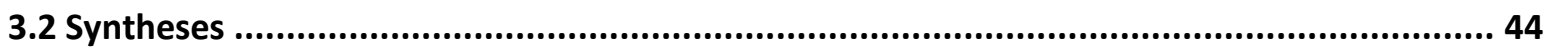

3.2.1 ATRP initiator immobilization on polyethylene terephthalate (PET) membrane ............................ 44

3.2.2 Dithiocarbamate immobilization on PET membrane ............................................................... 45

3.2.3 Surface-initiated ATRP of tert.-butly methacrylate on PET membrane ......................................... 45 
3.2.4 Hydrolysis of grafted poly(tert.-butly methacrylate) to poly(methacrylic acid)

3.2.5 Preparation of lysozyme/immunoglobin G imprinted membraned via UV-initiated grafting/-

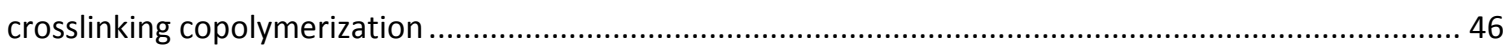

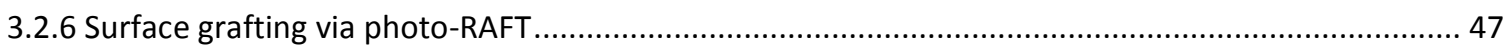

3.3 Characterizations .................................................................................... 47

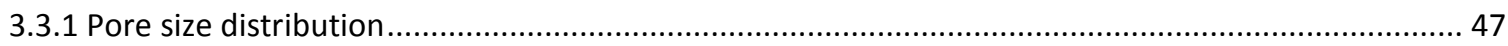

3.3.2 Liquid permeability measurement and subsequent analyses .................................................. 48

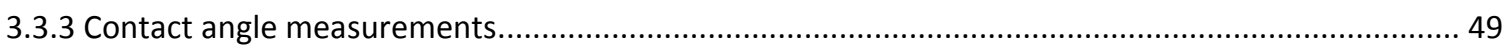

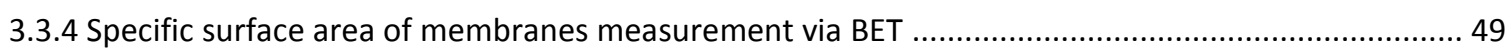

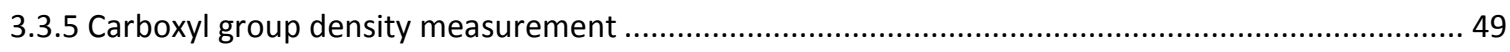

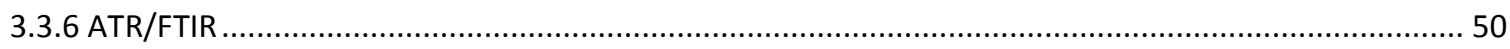

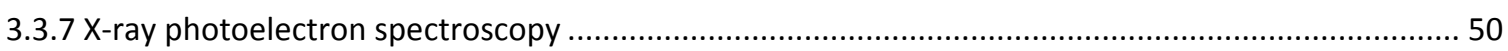

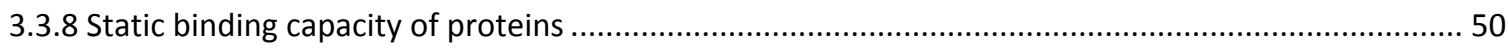

Chapter 4 Results and discussion ..................................................................... 52

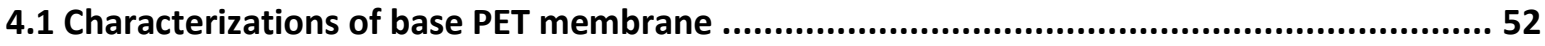

4.2 ATRP initiator immobilization on PET membrane ................................................... 54

4.3 Surface initiated ATRP on PET membrane ........................................................ 58

4.4 Hydrolysis of poly(tert.-butyl methacrylate) (PtBMA) to poly-(methacrylic acid) (PMAA) on PET membrane surface .......................................................................... 65

4.5 Optimization of UV-grafting/crosslinking on PET-g-PMAA membrane.............................. 75

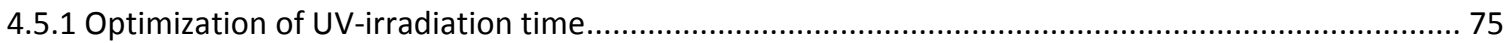

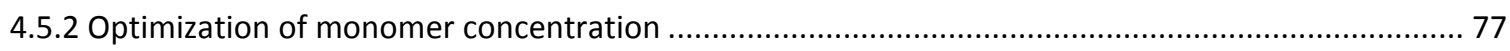

4.5.3 Optimization of UV-irradiation time with $0.3 \mathrm{~mol} / \mathrm{L}$ monomer concentration ................................ 78

4.6 Lysozyme imprinting on PET membrane....................................................... 80

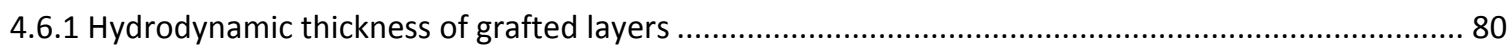

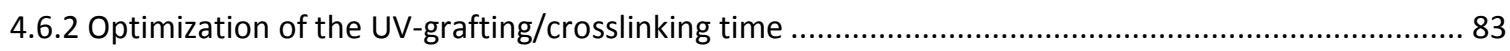

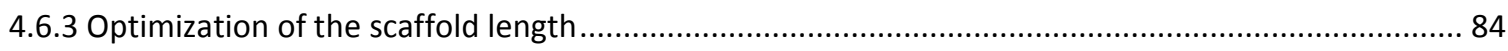

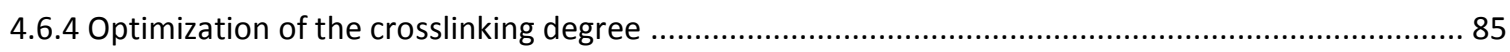

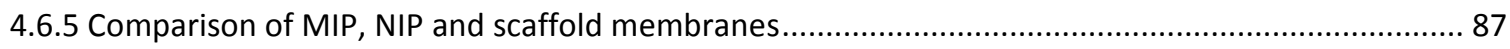

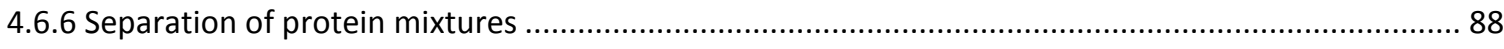

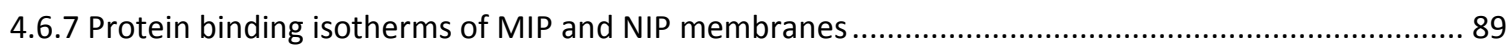

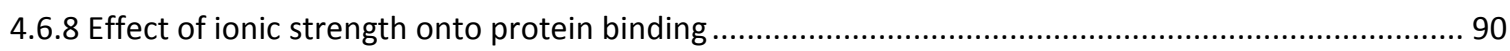

4.6.9 Introduction of more additive monomers during UV-grafting/crosslinking .................................. 92

4.7 Immunoglobin G imprinting on PET membrane ................................................95

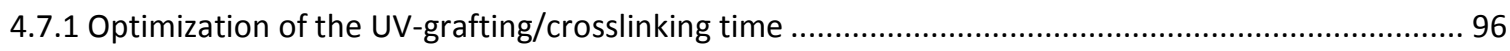

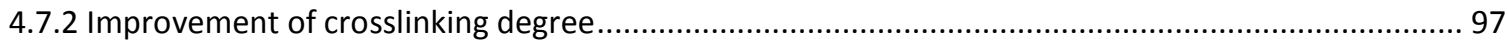

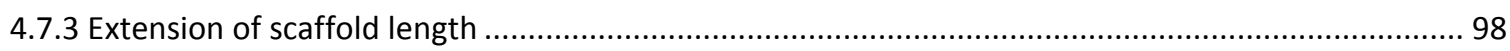

4.8 SI-ATRP and subsequent hydrolysis on cellulose membrane ....................................101

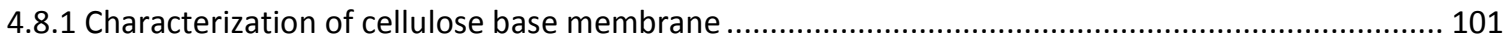

4.8.2 Surface functionalization via initiator immobilization and subsequent ATRP ............................... 103

4.8.3 Hydrolysis of PtBMA to PMAA on cellulose membrane........................................................... 105 


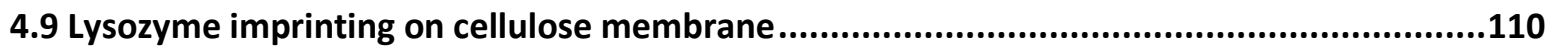

4.9.1 Characterizations of UV reactivity of PMAA-g-cellulose membrane............................................ 110

4.9.2 Optimization of UV-irradiation time with $0.3 \mathrm{~mol} / \mathrm{L}$ monomer concentration ............................... 111

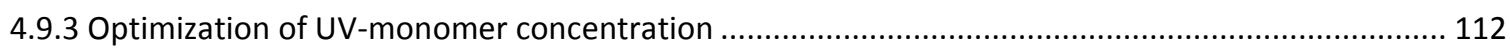

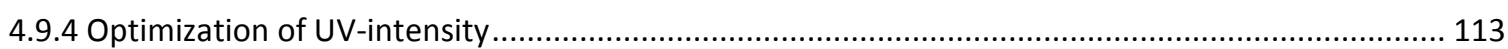

4.9.5 Optimization of UV-irradiation time under highest UV-intensity .............................................. 114

4.9.6 Optimization of crosslinking degree under highest UV-intensity.................................................. 116

4.9.7 Optimization of UV-irradiation under highest UV-intensity with 15\% degree of crosslinking .......... 118

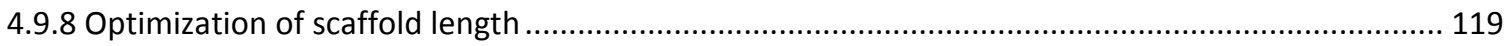

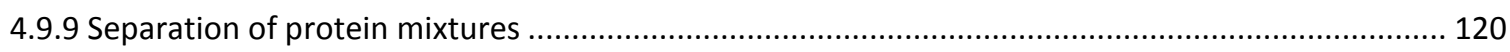

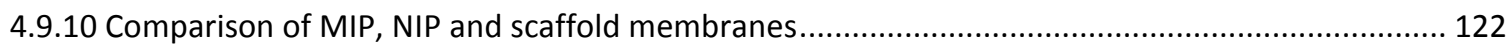

4.9.11 Protein binding isotherms of MIP and NIP membranes .................................................... 123

4.10 TE PET membrane immobilized with two "orthogonal" initiators..................................126

4.10.1 Immobilization of benzyl chloride group and subsequent characterization via ATRP .................. 127

4.10.2 Conversion of the benzyl chloride group to dithiocarbamate group ........................................ 128

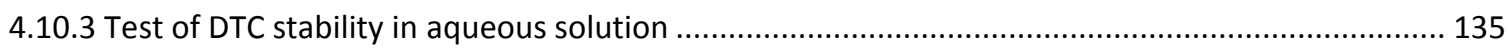

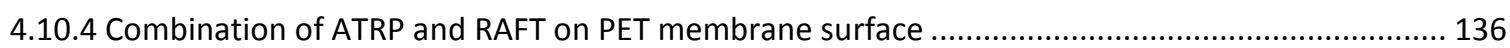

Chapter 5 Conclusions and outlook...............................................................141

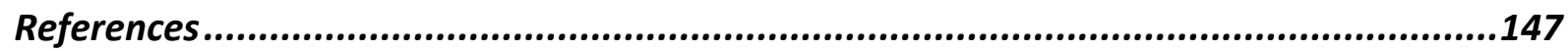

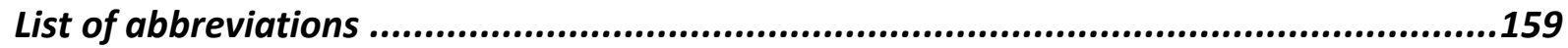

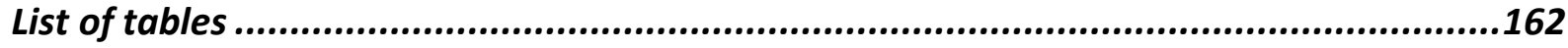

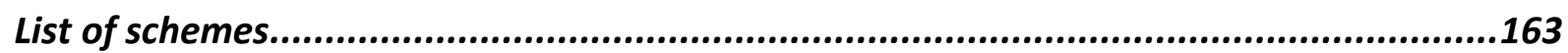

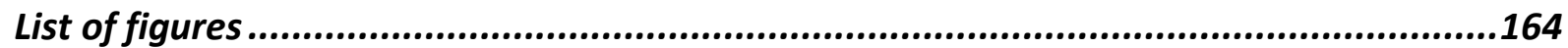

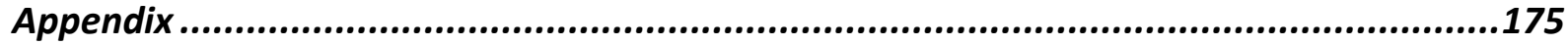

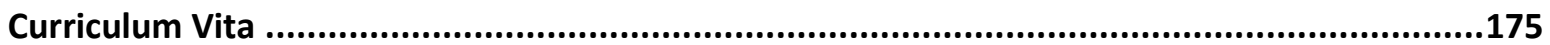

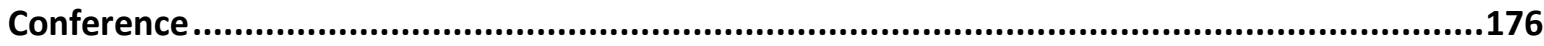

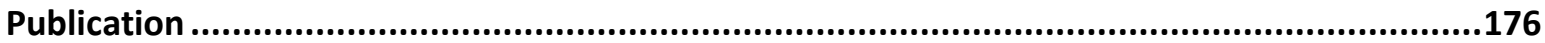




\section{Chapter 1 Introduction}

\subsection{Background and motivation}

Molecular imprinting is a generic, robust and cost-efficient technique to produce materials with specific recognition properties to target molecules or particles. This approach involves fixation of a complex between functional monomers or polymers and a template for the target in a polymer network via crosslinking or solidification. The template is then removed from the polymer and leaves behind the nanocavities possessing size, shape, and arrangement of functional groups which are complementary to the template. ${ }^{[1-3]}$ The molecular imprinting concept has been studied extensively, but mainly small molecule imprinting has been applied and developed successfully even to industrial scale. Since there is a strong need in areas such as diagnosis, separation, biosensor, and biomedicine to synthesize materials that can specifically recognize also biomacromolecules, protein imprinting has received considerable attention and has also shown extremely rewarding potential. Nevertheless, it is very challenging to obtain a molecularly imprinted polymer (MIP) for a protein; some obstacles are poor mass transfer or permanent template entrapment within the polymer, or inefficient imprinting due to conformational flexibility and complexity of the protein. Molecular imprinting can conventionally be classified by the strategies, with respect to the format of the MIP into bulk imprinting (3D) or surface imprinting (2D), and with respect to the (protein) template into full protein or epitope imprinting. ${ }^{[4]}$

The most common and successful protein imprinting strategy is surface imprinting, by which the imprinted binding sites are located at or near the surface of a base material. Surface imprinted materials on substrates demonstrated more potential for integration within different platforms, for instance as a sensor. The heterogeneity of imprinted binding sites is relatively high when only a fraction of the protein surface was imprinted, whereas this method facilitates the diffusion of the large protein in and out the polymer network, which is crucial for the material's functionality. ${ }^{[4-6]}$

Functional groups play a crucial role in the imprinting process since they contribute to binding to the target molecule before the actual imprinting (fixation) step. In conventional MIP procedures, functional monomers are used, and complex formation with a large 
template can lead to a manifold of different structures. Scaffold imprinting is a more specialized approach where instead of several functional monomer molecules in solution, several functional groups immobilized on a macromolecular chain or on a surface (i.e., on a "scaffold") are interacting with the template. ${ }^{[7]}$ The advantage of such pre-organization of potential recognition groups before the fixation step had been demonstrated in several cases, both for bulk and surface imprinting and including the synthesis of protein MIP either with protein ${ }^{[8]}$ or protein epitope ${ }^{[9]}$ templates.

In response to the still existing limitations of protein imprinting using established methods, a new strategy was addressed in this work by combining surface imprinting and scaffold imprinting via a novel two-step surface grafting method on commercial membranes. The aim was to use a surface-immobilized initiator which can be activated by two independent ("orthogonal") ways. In the first step, surface-initiated atom transfer radical polymerization (SI-ATRP) should lead to a layer comprising polymer chains of controlled lengths presenting functional groups for reversible protein binding (i.e., a grafted scaffold). After preorganization with a protein template, the second grafting step (UV-grafting/crosslinking) was intended to yield a cross-linked polymer layer interpenetrating with the scaffold chains and thus forming the imprinted sites (cf. Figure 1.1). The property of the imprinted structure can be optimized during the two-step surface grafting independently and respectively to divide one complex problem into two simpler problems.
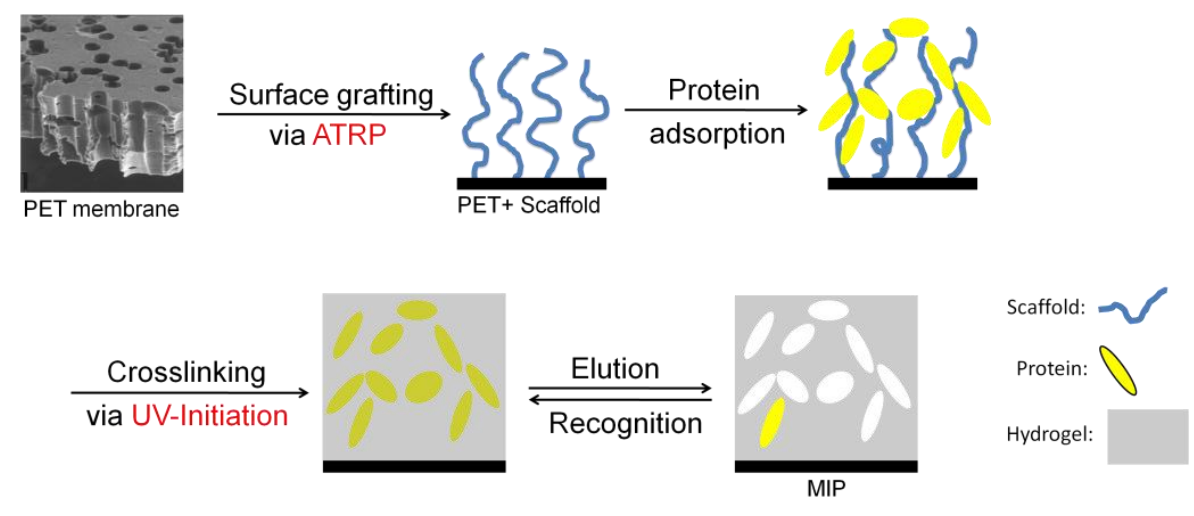

Figure 1.1 Protein imprinting via two step grafting using SI-ATRP and UV-initiated grafting/ crosslinking copolymerization on track-etched PET membrane surface. 


\subsection{Objective of the research}

The objective of this project is to produce protein imprinted composite membrane via a novel two-step grafting strategy in combination of ATRP and UV-grafting/crosslinking technology. To improve the performance of the protein imprinted composite membrane with high protein binding capacity and recognition property, at first the scaffold is grafted on the membrane surface with well defined architecture via SI-ATRP, then optimization of UVgrafting/crosslinking condition is performed.

To be more specific, the research tasks involve:

i. Study on the ATRP and the MIP techniques.

ii. Proposal of the novel two-step grafting strategy.

iii. Grafting of scaffold with well controlled architecture for protein orientation on TE PET membrane surface via initiator-immobilization and SI-ATRP.

iv. Optimization of the scaffold structure and the UV-grafting/crosslinking condition for the template lysozyme (Lyz) to improve the performance of the MIP composite membrane adsorber.

v. Application of the proposed technique for the protein with more complicate structure such as immunoglobulin $G(\operatorname{IgG})$ and for the membrane which has bigger surface area such as Cellulose R60 (Whatman).

vi. Evaluation of the performance of the protein imprinted composite membranes.

vii. Development of a novel TE PET membrane immobilized with two different initiators which can be initiated by ATRP and reversible addition fragmentation chain transfer polymerization (RAFT) respectively and independently. 


\subsection{Strategy of this dissertation}

ATRP is one of the most successful controlled polymerization techniques. SI-ATRP in combination with the adapted initiator immobilization provides a very powerful approach to modified surface with a well-defined polymeric layer in terms of chemical functionality and architecture. The combination of the MIP technique and membrane technology demonstrated a high potential for the commercial application in protein separation and recognition.

TE PET membranes with uniform cylindrical pores (diameter $675 \mathrm{~nm}$ ) at very narrow size distribution were used as substrates; these features were advantageous for the further characterizations because specific surface area was larger than with nonporous films and quantitative investigation of grafted polymer layer dry and hydrodynamic thicknesses was possible. ${ }^{[10-11]}$ TE PET membranes had also already been used successfully for the development of another novel protein selective adsorber based on linear grafted chains, which providing a layer where multiple interactions with special amino acid-selective side groups led to pronounced protein affinity and selectivity. ${ }^{[12]}$ SI-ATRP in combination with the adapted initiator immobilization provides a very powerful approach to modify surfaces with a well-defined polymeric layer in terms of chemical functionality and architecture. ${ }^{[13]}$ This method had also already been used successfully to prepare well-controlled grafted functional polymer layers in the pores of TE PET membranes. An alkyl bromide as ATRP initiator had been immobilized on the membrane surface in adjustable density through oxidative PET hydrolysis and subsequent two-step coupling (Scheme 1.1). ${ }^{[1,14]}$ In a controlled slow ATRP reaction, the $\mathrm{C}$ - $\mathrm{Br}$ structure will always remain at the polymer chain end (Scheme 1.2). It is also known that the photolysis of aliphatic bromides can be used to selectively initiate a graft copolymerization on polymer surfaces. ${ }^{[15]}$ Therefore, the concept of this work was to use SI-ATRP as first step to graft a macromolecular scaffold and to use photo-initiated grafting via residual initiator sites on the surface and $\mathrm{C}-\mathrm{Br}$ groups at the chain ends of the scaffold to obtain a surface-anchored MIP layer.

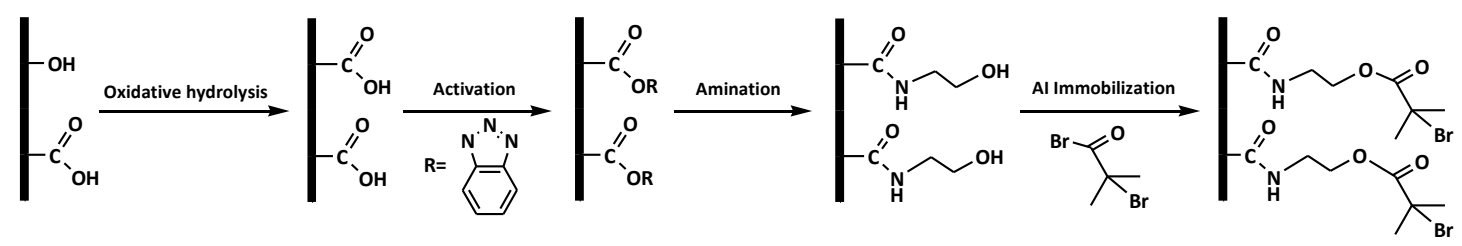


Scheme 1.1 Immobilization of ATRP initiator on TE PET surface.

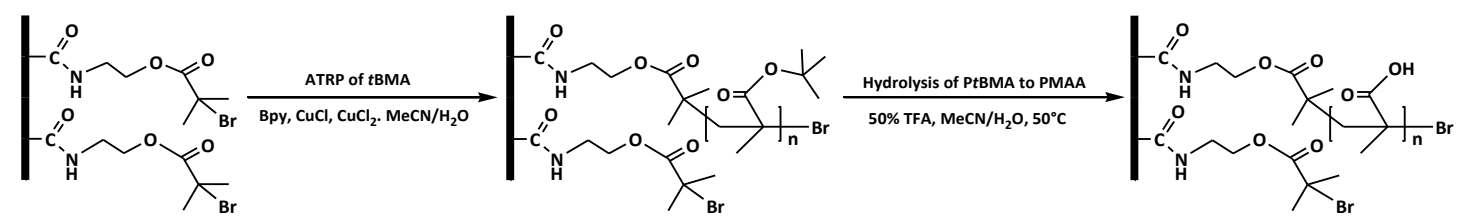

Scheme 1.2 SI-ATRP of PtBMA and subsequent hydrolysis to PMAA on TE PET surface.

A novel two-step grafting strategy for protein imprinting was proposed toward the synthesis of protein imprinted structure on membrane surface. At first Lyz imprinted TE PET membrane was developed. ATRP initiator $\alpha$-bromoisobutyryl group was immobilized on the PET membrane surface. The surface-immobilized scaffold poly(methacrylic acid) (PMAA) was obtained by hydrolysis of grafted poly(tert.-butyl methacrylate) (PtBMA), which had been synthesized via SI-ATRP of tert.-butyl methacrylate (tBMA) (cf. Scheme 1.2). In order to better control the grafting of PtBMA, the factors that affect the SI-ATRP such as reaction time, solvent, component of the catalyst system, concentration of the monomer have been investigated. The hydrolysis condition of PtBMA to PMAA was also optimized. After template Lyz binding to the scaffold which is facilitated by ionic interactions, imprinting in a polyacrylamide (PAAm)-based hydrogel layer was realized via UV-initiated grafting/crosslinking copolymerization using mixtures of acrylamide and $\mathrm{N}, \mathrm{N}^{\prime}$ methylenebisacrylamide (MBAA). Subsequently, the template was eluted from the hydrogel layer to yield a porous membrane adsorber with MIP nanocavities. The protein binding capacity and selectivity were measured with Lyz and cytochrome $\mathrm{C}$ which have very similar size and isoelectric point. To improve the performance of the MIP composite membrane, the length of the scaffold PMAA, UV-irradiation time, UV-intensity, degree of crosslinking were investigated in detail. The imprinted technique was extended by application of the IgG imprinting on PET surface and the Lyz imprinting on cellulose R60 membrane surface.

In order to improve the control of UV-grafting/crosslinking, a novel membrane support which can be initiated by ATRP and RAFT respectively and independently was developed. Iniferter dithiocarbamate (DTC) was immobilized on PET membrane surface by conversion of the immobilized ATRP initiator benzyl chloride group in acetonitrile (MeCN) using a phase transfer catalyst tert butyl ammonium bromide (TABA). The conversion of two initiators was 
characterized via contact angle and XPS. The initiation independence of these two initiators was investigated via different initiation subsequence of ATRP and RAFT. 


\section{Chapter 2 Theory}

\subsection{Molecular imprinting}

Molecular recognition is one of the most fundamental processes in nature which rests on the molecular information stored in the interacting partners. ${ }^{[16]}$ This simple but elegant mechanism is found in a variety of biological processes, including antibody/antigen recognition in the immune system, enzymatic catalysis, signal transduction, and nucleic acid interactions such as replication, transcription, and translation. ${ }^{[6]}$ Natural recognition is composed of non-covalent forces, such as hydrogen bonding, van der Waals force, $\pi-\pi$ interaction, electrostatic interaction, and hydrophobic interaction. ${ }^{[17]}$ The complementarity between receptor and substrate was described by Emil Fischer first as the "lock and key model" in 1890s. ${ }^{[1]}$

The natural recognition elements such as antibody-antigen interaction have inherent disadvantages and limitation of the application in harsh environment including poor chemical, physical, and long-term stability. ${ }^{[18]}$ The single functionality and complicated synthesis process normally consuming lots of time and money of natural recognition agents are the other main obstacles to use it. In order to overcome the above weaknesses, development of synthetic receptor has attracted more and more attention to mimic the high recognition property of nature elements. One of the most successful techniques is molecular imprinting, which is based on creation of artificial recognition sites in synthetic polymers. Molecular imprinting has a long history since the beginning of last century, but the main breakthroughs of this science subject have occurred since 1990s. In 1930s, the selective effects were first explained in terms of a template effect by Polyakov (Kiev). ${ }^{[6]}$ Linus Pauling recognized the importance of shape selectivity during the interaction between antibody and antigen in the immune system. "Complementariness" concept proposed by Pauling inspired the starting work of molecular imprinting. In 1966 Mosbach reported immobilization of enzymes and cells by entrapment in polyacrylamide gel networks via no covalent interaction, which laid the foundation of the current field specially for biomolecule imprinting. ${ }^{[19]}$ The imprinting of organic polymers by covalent approach was first reported by Wulff's group in 1972, which involves covalent complex formation between template and the surrounding polymer. ${ }^{[20]}$ 
Molecular imprinting involves fixation of a complex between functional monomers or polymers and a template for the target in a polymer network via crosslinking or solidification. The template is then removed from the polymer and leaves behind the nanocavities possessing size, shape, and arrangement of functional groups which are complementary to the template (Figure 2.1).
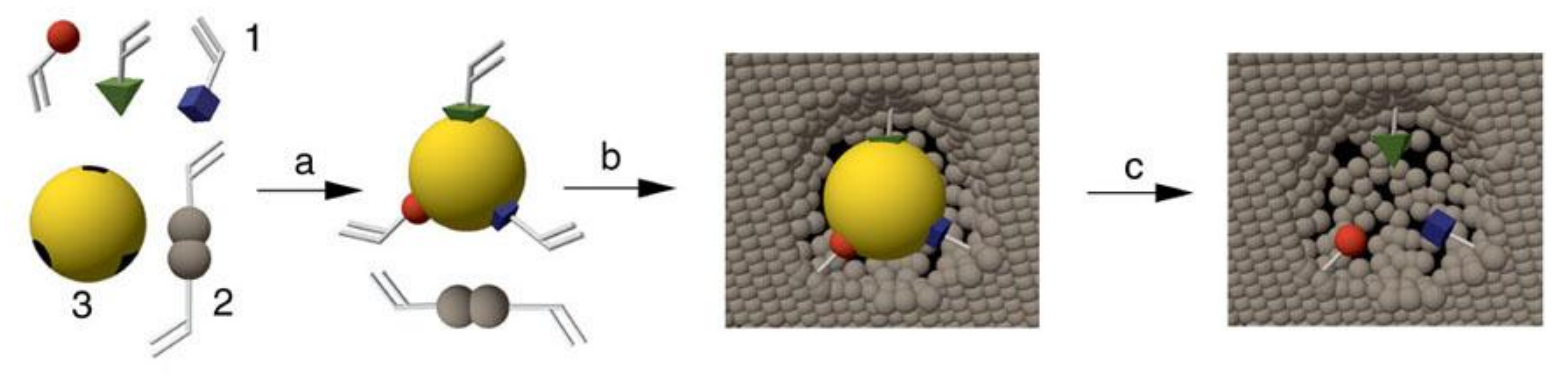

Figure 2.1 Schematic representation of molecular imprinting process containing functional monomers (1), crosslinker monomer (2), and template via complex by prepolymerization (a), polymerization with crosslinker (b), and template extraction (c). ${ }^{[21]}$

The template of imprinting is typical small organic molecules by covalent imprinting, the nocovalent interaction method has extended the template range from small molecules to large biomolecules such as protein, DNA, and RNA. Acrylamide and (meth)acrylic-based monomers remain today still the most important and universal monomers for molecular imprinting. The structure of the polymer matrix is crucial in the imprinting process. As the specific structure of the imprinted cavity is not determined by the template molecule, but by the fixed arrangement of the polymer chains, the optimization of the polymer structure is extremely important such as polymerization degree and crosslinking degree. Wulff proposed the requirement to the polymer structure with the following properties: ${ }^{[1]}$

1. Stiffness of the polymer structure enables the cavities to retain their shape even after removal of the template, this giving high selectivity.

2. High flexibility of the polymer structure works against the above, but is essential for the kinetics, to give rapid equilibration with the substrate be embedded.

3. Good accessibility of as many cavities as possible in the highly crosslinked polymer can be achieved forming a particular polymer morphology. 
4. Mechanical stability of the polymer structure is of great importance for many applications, for example, for use in an HPLC column at high pressure or as a catalyst in a stirred reactor.

5. Thermal stability of the polymers enables them to be used at higher temperatures, at which the kinetics are considerably more favorable.

Molecular imprinting of small molecule has been intensively investigated and well developed. There are now commercial tailor-made products by the companies such as MIP Technologies AB (Lund, Sweden), Semorex (USA and Israel) and POLYIntel (Val de Reuil, France). Macromolecular imprinting has received more and more interest from the scientific community since last ten years, which uses the template molecule with a MW > $1500 \mathrm{Da}$. Protein imprinting has received more and more attention, however there are still challenging obstacles in protein imprinting, the potential reward is also very obvious. ${ }^{[6]}$ 


\subsection{Protein imprinting}

Protein is polypeptide consisting of 20 different L- $\alpha$-amino acids (Figure 2.2). Structure of protein has four levels: primary structure, secondary structure, tertiary structure, and quaternary structure. The primary structure is linear polypeptide chain with a carboxyl terminus and amino terminus. Due to the hydrogen bonds of polypeptide chain, the secondary structure of protein was formed in alpha helix and beta sheet. Under the driven force of hydrophobic interaction, alpha helix and beta sheet were folded into a compact globule form and stabilized by the tertiary interactions such as salt bridge and disulfide bond. Multi tertiary proteins combine together via non-covalent interactions to form the quaternary structure of protein (cf. Figure 2.3). $3 \mathrm{D}$ structure of protein and the surface residues (functionalities) determine the chemical, physical, and biological properties of proteins. Surface residues of protein are amino acid with partly protonated amino groups and deprotonated carboxyl groups determined by the $\mathrm{pK}_{\mathrm{a}}$ and $\mathrm{pK}_{\mathrm{b}}$ values and the environment. The isoelectric point $(\mathrm{pl})$ is the $\mathrm{pH}$ value at which the protein has no net electrical charge. ${ }^{[22]}$

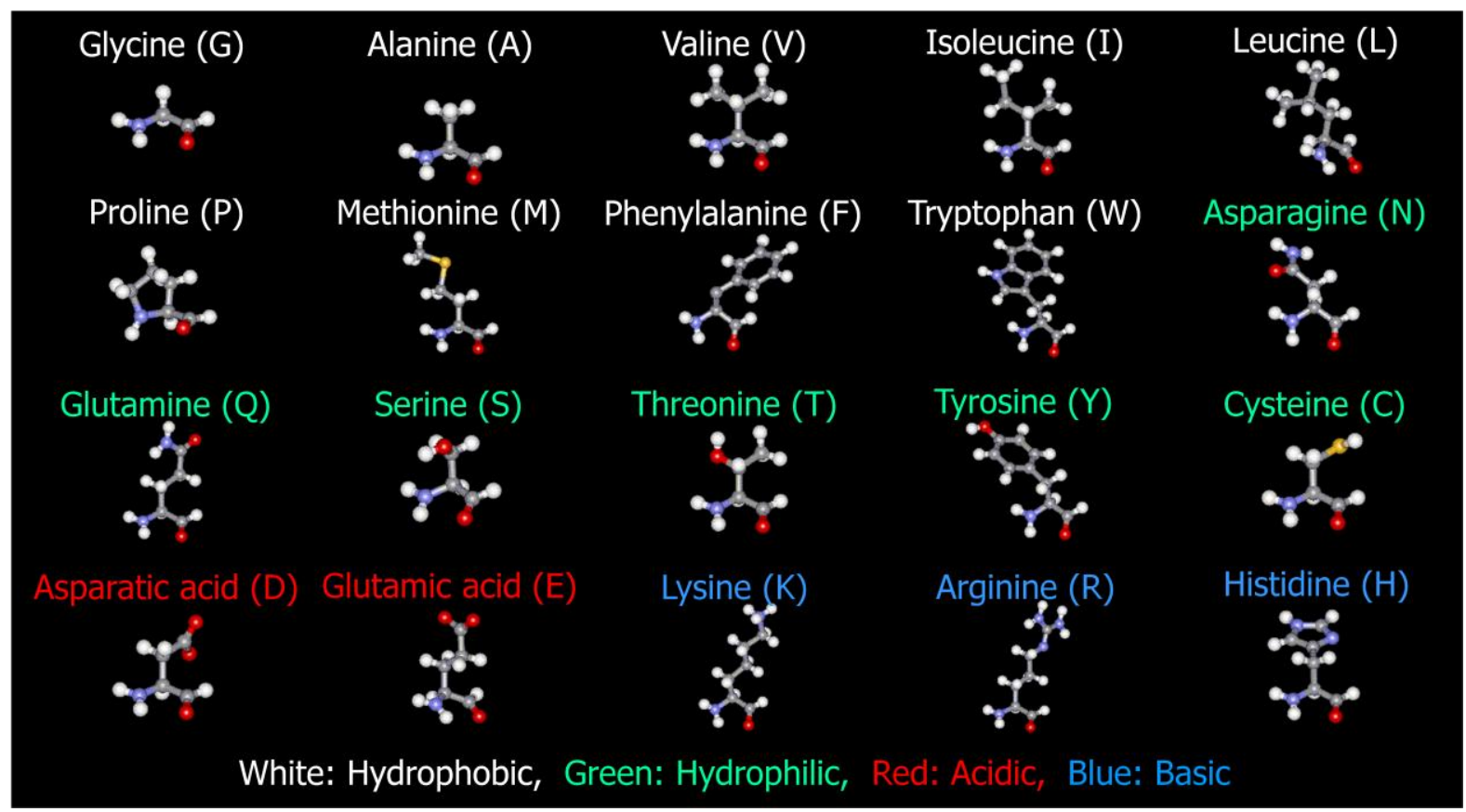

Figure 2.2 20 L- $\alpha$-amino acids. ${ }^{[23]}$ 


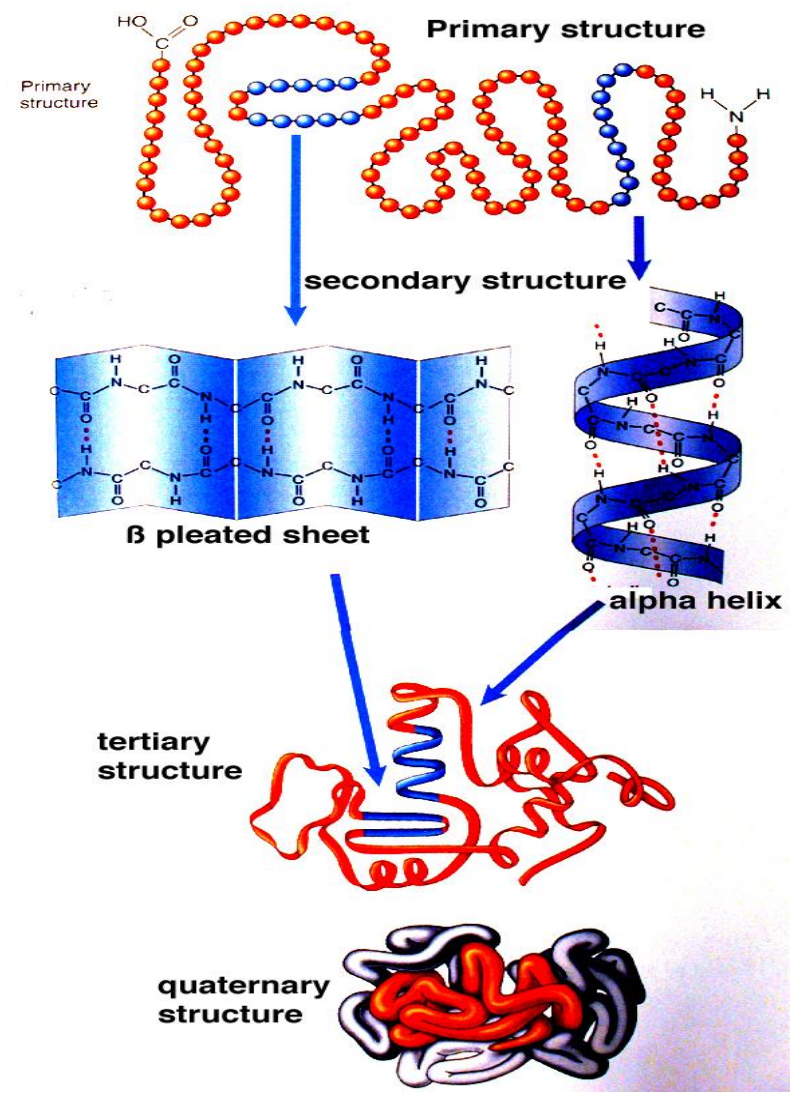

Figure 2.3 Four level structure of proteins ${ }^{[24]}$

\subsubsection{Obstacles of protein imprinting}

Most selective protein binding in laboratory is dependent on antibodies to achieve specific protein entrapment in assays and in isolation, extraction, and biosensors. However, such kind of techniques is notoriously expensive and often suitable only for single use due to the fragility and labile nature of the protein recognition element involved. Protein imprinting which is extended from the small molecular imprinting is seen to be a good alternative technique for the typical biological technique with inexpensive, robust, and reusable properties. Despite the obvious advantages of protein imprinting, some hindrances for protein imprinting have been also addressed, most of them is due to the inherent properties of protein, such as big size, complexity, conformational flexibility and solubility. ${ }^{[4]}$

The traditional polymer network for small organic molecule imprinting is relatively dense with small pore size in order to retain the binding sites created during polymerization. However, this leads to the poor mass transport and permanent entrapment by protein imprinting, because the protein is much bigger than the template of typical small molecule 
imprinting. The slow leakage of the template and poor diffusion of the analyte can result in poor recognition properties.

Proteins are complex biopolymers composed of the linear sequences of amino acids that present a large number of potential recognition sites having lot of different residues on the protein surface. Different portions of the protein exhibit distinct physiochemical properties. To achieve a relatively easy on/off binding event where the protein template can be removed with minimal damage to the three-dimensional (3D) cavities, a no-covalent recognition process is favored to form multiple weak interactions which tend to favor more nonspecific binding.

The conformation of protein is sensitive to the environment, such as temperature, ionic strength and $\mathrm{pH}$. Most of the imprinting conditions is non-physiological, it results in changes of protein template structure and leads to conformation difference from their natural form. Therefore, when rebinding is performed later in the physiological conditions, specific recognition of the template may be low.

Solvent is also a key issue of success in the protein imprinting to date. A good solvent for imprinting should have no interference on the monomer/polymer-template interaction and allow complete miscibility between all the constituents. Protein is often insoluble or unstable in the typical aprotic organic solvents for small molecule imprinting, and protein are completely miscible in aqueous solutions which is far from the ideal traditional solvent, because water will affect the physical interaction between monomer and template such as hydrogen bonding which plays an important role by the non-covalent imprinting.

\subsubsection{General strategies of protein imprinting}

In spite of these above obstacles, protein imprinting is proposed to have a high rewarding potential and was intensively investigated via different approaches which can be commonly categorized as bulk, surface, epitope and scaffold. From 2005 more and more works focused on the protein imprinting, of those more than half of the protein imprinting is based on the strategy of surface imprinting. Bovine serum albumin (BSA), lysozyme (Lyz) and bovine hemoglobin $(\mathrm{BHb})$ are the most common templates. Acrylamide (AAm), methacrylic acid (MAA), acrylic acid (AA), aminophenylboronic acid (APBA) and $\mathrm{N}$-isopropylacrylamide 
(NIPAAm) account for nearly $60 \%$ monomers used for protein imprinting. More than $80 \%$ of crosslinkers are $\mathrm{N}, \mathrm{N}^{\prime}$-methylenebisacrylamide (MBAA) and ethylene glycol dimethacrylate (EGDMA). ${ }^{[6]}$

\subsubsection{Bulk imprinting (3D)}

Bulk imprinting has been applied successfully by the small molecule imprinting and is the most straightforward approach to protein imprinting. The advantage of bulk imprinting is the 3D binding sites formed for the entire protein, which is similar to the typical small molecular imprinting. The obvious limitation of bulk imprinting strategy is the poor mass transport of protein, but it is a fundamental requirement of any imprinting procedure that the template protein can be freely removed and reintroduced to the imprinted sites. To overcome such problem, the porosity and the pores size are increased by bulk imprinting via reduction of the crosslinking degree, however, this normally leads to a loss of the recognition property.

In 1990s, Hjerten and his coworkers developed PAAm gel to recognize the proteins. The polyacrylamide gel is biocompatible and neutral, these factors were considered to reduce the non-specific interaction between PAAm and proteins. The imprinted gel was pressed through a sieve, and the resultant particles were packed into a column for chromatography. The proteins were adsorbed in sodium phosphate and removed in a solution of acetic acid and sodium dodecyl sulfate (SDS). The PAAm gel made of acrylamide and MBAA at different ratios were imprinted with $\mathrm{BHb}$, cytrochrome $\mathrm{C}(\mathrm{CyC})$ and human transferrin, ${ }^{[25]}$ and later for human growth hormone (HGH), RNase and horse myoglobin (HMb). ${ }^{[26]}$ An imprinted PAAm gel with simultaneous responsibility to three proteins $\mathrm{CyC}$, Lyz and human hemoglobin was also investigated. ${ }^{[27]}$ It was hypothesized that a large number of weak interactions form between the gel and the protein, giving an overall strong interaction and hence the success of the imprint. This is in opposition to traditional imprinting theory that suggests that fewer strong bonds are better than numerous weak ones.

AAm and MBAA are still the most usual components by imprinting to date. PAAm gel was later modified by the incorporation of chargeable functional monomers in the polymeric backbone to improve the property of imprinted hydrogel, such as negative MAA by Pang et al., ${ }^{[28]}$ MAA and positively charged $N$-[3-(dimethylamino)propyl] methacrylamide by Huang et al. ${ }^{[29]}$ 
Template removal is a crucial step by protein imprinting. The percent of the initial template entrapment after crosslinking is much higher than that of small molecule imprinting. The most common methods to remove the template include SDS/acetic acide by Hjerten and his coworkers, SDS/NaOH by Pang et al., and aqueous $\mathrm{NaCl}$ by Ou et al. ${ }^{[30]}$ It should be noted that a suitable compromise between effective template removal and integrity of the binding sites is prior to recognition studies.

In considering the limitation of diffusion, solubility and conformational flexibility of protein, gel technologies is the typical approach for protein bulk imprinting, which is normally synthesized the imprinted structures in aqueous solution with a low crosslinking degree in order to improve the protein transport in the polymeric network, but it can also lead a poor maintenance of the imprinted structure for long period. Additionally, the harsh condition of template removal can also affect the imprinted gel structure and reduce the recognition property. As a result, the alternative approaches have received more and more interest. ${ }^{[4]}$

\subsubsection{Surface imprinting (2D)}

Surface imprinting is the most common protein imprinting strategy, by which synthesized imprinted binding sites are located at or very near the surface of the polymer. Synthesis of thin polymer film is a simple strategy of surface imprinting using similar approach as by bulk imprinting. Orientation of the protein template on the surface of a substrate with subsequent polymerization is another promising method to achieve the surface imprinting. Surface imprinting allows free access to the binding sites for protein, thereby minimizes template size concerns. Due to the presence of the support, the imprinted structure is more physically robust and easily integrated with other sensor platforms. However, only portion of the protein surface (anchor point) was imprinted, a reduction of specificity cannot be avoided. ${ }^{[6]}$

Shi et al. developed surface imprinting via radio frequency glow-discharge (RFGD) plasma deposition to form thin polymer films around the protein template with disaccharide molecules. The template proteins were fixed onto a mica surface and exposed to a disaccharide solution. During sample dehydration, multiple hydrogen bonds are formed between the hydroxyl groups of disaccharide and the polar residues on the surface of the protein. After RFGD plasma deposition, the covalent bonds between disaccharides were 
formed, this structure was then fixed on a support. The thin film with protein imprinted cavities was produced by subsequent removal of the mica and protein. This strategy demonstrated an excellent property for BSA, IgG and Lyz recognition. Impressively, the selectivity of Lyz to RNase by Lyz imprinted film reached 20, even both proteins have similar size and isoelectric point. ${ }^{[31]}$

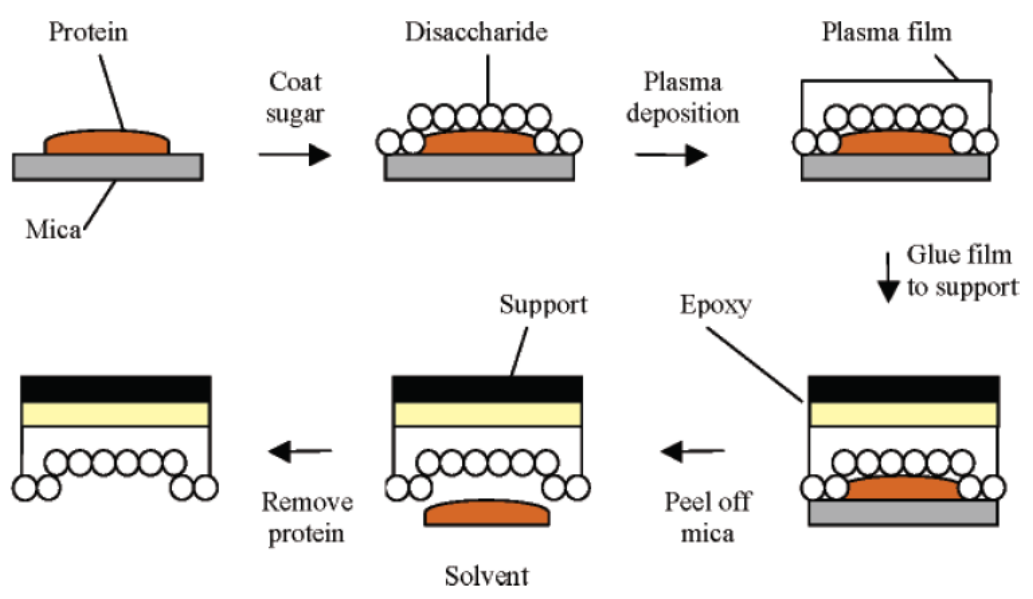

Figure 2.4 Surface imprinting of protein by Shi et al. via RFGD plasma deposition.

$\mathrm{Hu}$ et al. developed a novel strategy in combining surface protein imprinting and photo crystals for preparation of BSA detection sensor with self-reporting features. The imprinted hydrogel on highly ordered silica colloidal particles surface which can be rapidly analyzed by readable optical signals. Prior to photo polymerization, a protein template was coated onto the surface of $\mathrm{SiO}_{2}$ colloidal particles (after fabrication via silica colloidal crystal templating) by spin-coating. Then, a dilute aqueous solution of saccharose was spin-coated as an overlay around the protein molecules to form a very thin sugar shell, which could serve as a protection layer to prevent the denaturation of the imprinted protein during the formation of the hydrogel by radical polymerization in ethanol containing a mixture of MAA, EGDMA and 2,2-azobisosobutyronitrile (AIBN). After removal of silica nanoparticles and the imprinted protein, a hierarchical hydrogel film with photonic crystal structure and "footprints" of the imprinted protein on the surface of the macroporous gel was obtained. ${ }^{[32]}$ Protein imprinting was integrated with living radical polymerization such as RAFT. The living radical polymerization can synthesize more monodisperse polymers over the traditional free radical polymerization, which demonstrates a potential ability to minimize heterogeneity of inner morphology and binding site affinities of the imprinted polymers. Qi et al. modified the 
surface of polystyrene beads with an iniferter DTC and synthesized polyacrylamide based thin film for recognition of Lyz. In comparing with the imprinted structure via traditional thermal free radical polymerization, the imprinted structure via RAFT demonstrated obvious better recognition properties with higher imprinting factors (12.8 vs. 2.6$)$ and selectivity (>10 vs. <1). ${ }^{[33]}$ One year later, the same group developed Lyz imprinted polymer layer on magnetic particles via ATRP in aqueous solution, the highly selective adsorption and recognition to Lyz was demonstrated by the separation ability of the magnetic imprinted material to template protein, and it has been used for quick and direct separation of Lyz from the mixture of standard proteins and real egg white samples under an external magnetic field. ${ }^{[34]}$ Controlled/living radical polymerization is a promising technique to improve the property of the imprinted structures.

\subsubsection{Epitope imprinting}

In nature, antibody-antigen interactions depend on the recognition between the antibody and an antigenic site of the protein-the epitope. The epitope is a short amino acid sequence complementary to binding site of antibody. In the field of molecular imprinting, Rachkov and Minoura applied this strategy to develop the epitope imprinting for protein recognition. Instead of the whole protein, a short peptide sequence, often exposed the protein surface, was used as a template during the imprinting process. Once the matrix has been polymerized the resultant imprinted material should be able to recognize and bind the whole protein. ${ }^{[5]}$

Rachkov and Minoura created an imprinted polymer specific for the nonapeptide oxytocin by a polymer imprinted with a tertrapeptide YPLG via a conventional small molecule imprinting by using the MAA and EGDMA in organic solvent. Oxytocin and YPLG have the same three amino acid sequence at the C-terminus. This allowed to form the specific and strong interactions with part of the protein, rather than relying on numerous weak interactions all over the whole protein, thus increasing specificity and affinity. The imprinted polymer was characterized via packing in a HPLC column and the selective adsorption was investigated by ionic interaction, $\mathrm{pH}$ and ratio of the monomer to the template in details. ${ }^{[35]}$

Shea et al. developed the epitope imprinted thin films to recognition cytochrome $\mathrm{c}$, alcohol dehydrogenase, and BSA via imprinting the epitope AYLKKATNE, GRYVVDTSH, and 
VVSTOTALA, respectively. At first the epitopes were immobilized on the glass or silica surface through alkylation, oxidation, and peptide immobilization. The imprinted polymer was achieved as outlined in Figure 2.5. The epitope immobilized surface was exposed to the reaction solution including initiator, functional monomers, and crosslinker, the polymerization was initiated by UV-light. After polymerization, the glass substrate or silicon wafer was removed to obtain the imprinted polymer film. Protein affinity was established by incubating imprinted films in a mixture of proteins. After elution the protein uptakes by MIP films were characterized by SDS-PAGE. ${ }^{[36]}$

A)

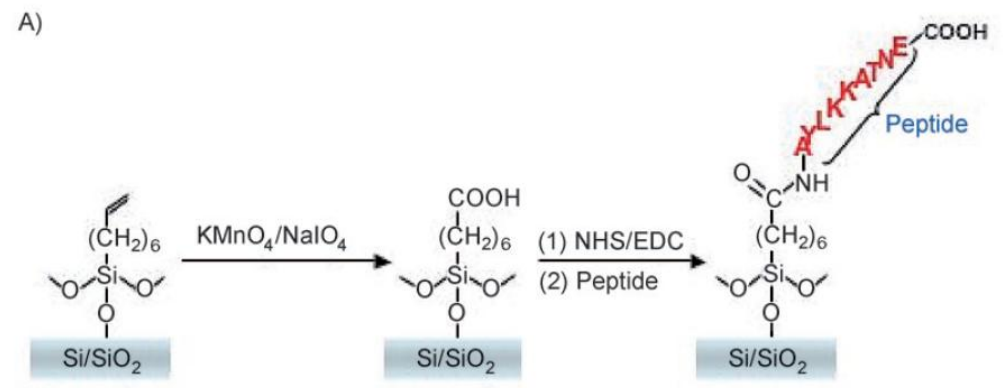

B)

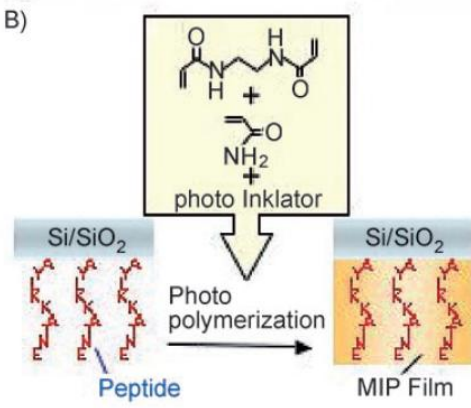

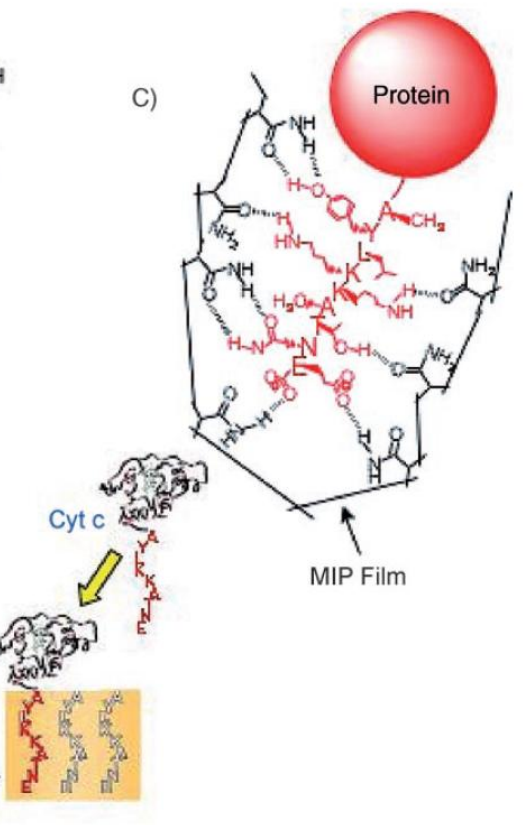

Figure 2.5 Protocol for epitope imprinting for protein CyC recognition by Shea et al. ${ }^{[36]}$

Shea et al. have first studied the property of epitope imprinted structure in vivo. The imprinted nanoparticle was synthesized by polymerization of multi functional monomers in presence of the template peptide melittin, a 26 unit cytolytic peptide that is the principal component of bee venom. First of all, the biocompatibility was tested via intravenous injection of the MIP-nanoparticles into the bloodstream of the mice. After two weeks, there was no significant difference in body weight between the injected and the control mice. The specific recognition and neutralization of the imprinted nanoparticle to toxic melittin was indicated by the intravenous injection of the melittin imprinted nanoparticles into the melittin infected mice which resulted in a significant reduction of the mortality in comparing 
with the mice injected with non melittin imprinted particles and the mice without particle injection. $^{[9]}$

Epitope imprinting has shown some advantages in comparison with bulk imprinting and surface imprinting, the main obstacles to successful imprinting of protein: size, complexity and conformational flexibility have been minimized through switch the macromolecule imprinting to small molecule imprinting. The epitope imprinting allowed to use the polymer network with high crosslinking degree without consideration of the macromolecular template' removal. The limitation of protein transport by the binding process can be minimized by combining epitope imprinting with the surface imprinting strategy; nevertheless, the binding capacity will be somehow reduced by this approach. The epitope imprinting uses the small molecular peptide as the template and natural antigen-antibody interaction for protein recognition, which can minimize the influence of the complexity of the protein and improve the specific property of the imprinted structure. The small peptide is much less sensitive to the environment than protein; the imprinting can also take place in organic solution just like traditional small molecular imprinting without consideration of solubility and conformation change. Usually, short peptides are cheaper than the proteins, but some synthesis of the template peptide is difficult. Overall, the epitope imprinting seems to be a good candidate for protein recognition by mimicking the natural technique.

\subsubsection{Scaffold imprinting}

Monomer elements play an important role in the imprinting process since each element causes interactive binding to the target molecule before imprinting polymerization. Therefore, the availability of scaffolding elements that can bind to a template molecule becomes a key element in specific recognition of the template molecule for imprinted materials. To obtain the imprinting structure with high recognition property, scaffold imprinting has been developed. The scaffold monomers, which have multi binding functionalities to the template to form more sophisticated recognition interactions, are used during the imprinting instead of the conventional single functional monomer. Scaffold polymer is a good extension of scaffold monomer, which having multi-complexible groups in a single macromolecule chain, this can lead to form more stable prepolymerization complex. The cooperative interactions of these multimers, monomers and polymers, with template 
are predicted to generate imprinted polymers with higher affinity and capacity for the target molecule. ${ }^{[7]}$

For the conventional small molecular imprinting, scaffold monomers with multi scaffold elements were used to improved the imprinting property such as urea, ${ }^{[37]}$ ethylenediaminetetraacetic acid (EDTA) / diethylenetriamine pentaacetic (DTPA), ${ }^{[38]}$ and cyclodextrin. ${ }^{[39]}$ Scaffold polymers were successfully used for the small molecule imprinting. Kofinas et al. investigated the imprinting of glucose phosphate mono-sodium salt by using a polymer scaffold poly(allylamine hydrochloride), different crosslinkers epichlorohydrin, ethylene glucol diglycidyl ether, and glycerol diglycidyl ether were evaluated by the effectiveness of imprinting for glucose. ${ }^{[40]}$ Fuji et al. developed a L-glutamine imprinted membrane based on non-covalent bonding by using scaffold polymer via phase inversion. Hydrogen bond between the synthetic polyamide was used as imprinting media for chiral recognition of L-glutamine scaffold. The imprinted membrane demonstrated a higher affinity to L-glutamine than D-glutamine, L-glutamic acid, and D-glutamic acid. Recognition of Lglutamine was investigated via membrane filtration and quartz-crystal microbalance. ${ }^{[41]}$

$\mathrm{Mi}$ and his coworkers synthesized scaffold polymers named assistant recognition polymer chains (ARPCS) for protein imprinting, as monomer extender enhanced the recognition specificity (Figure 2.6). Two types of ARPSs integrated with positive and negative charge respectiviely were evaluated through the template protein pig cylophilin 18 affinity after imprinting. At first, the template protein was selectively assembled with ARPCs from the library, which consisted of numerous limited-length polymer chains with randomly distributed recognition sites for assembling with protein and immobilization sites. These protein/ARPSc complexes were adsorbed by porous polymeric beads and immobilized by crosslinking polymerization. After removal of the template protein, binding sites that were complementary to the target protein in size, shape, and position of recognition groups were exposed, and their conformation was preserved by the crosslinked structure. It is believed to be the first time that the imprinted polymers were used to purify target protein from cell extraction. $^{[8,42]}$ 


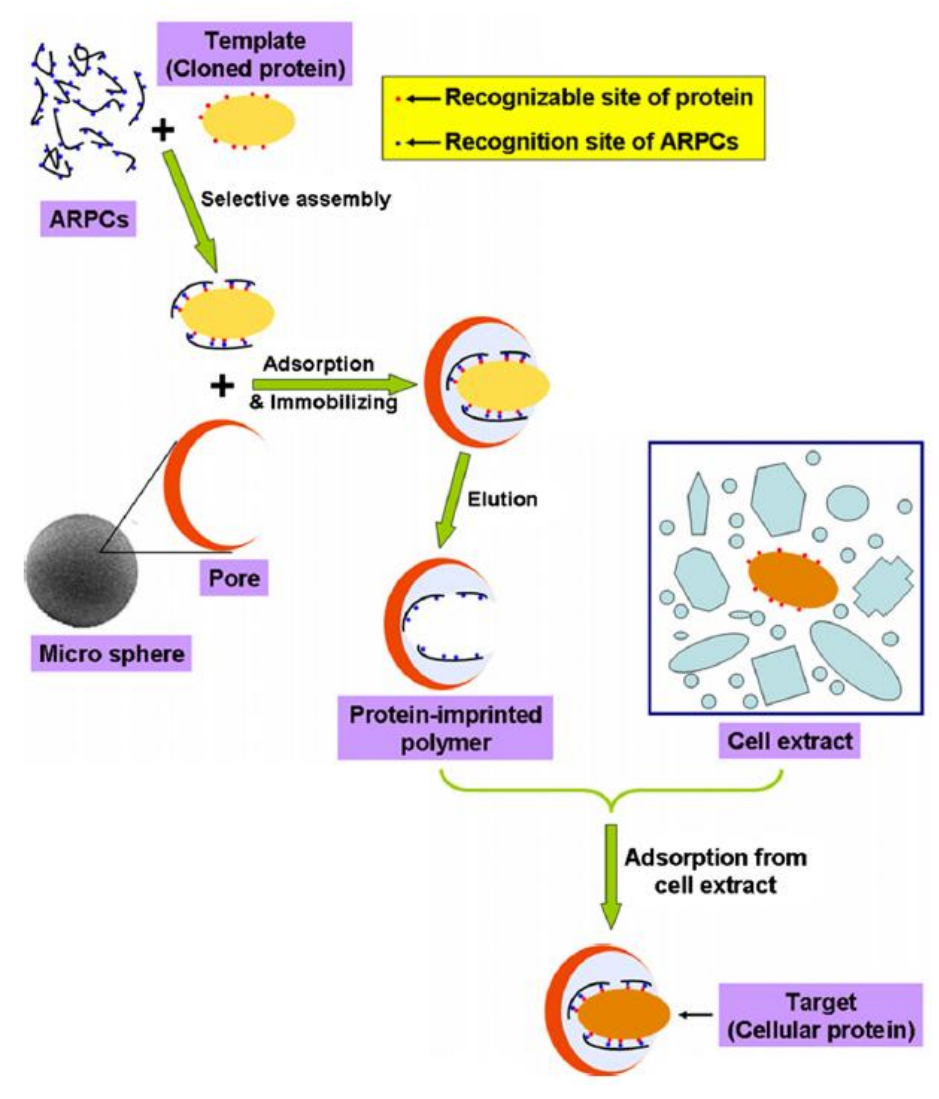

Figure 2.6 Protein scaffold imprinting with assistant recognition polymer chains from Mi and coworkers. $^{[8]}$

Scaffold elements can be classified into two categories of monomer and polymer which are integrated with multiple binding sites. The obvious obstacle for scaffold imprinting is the difficult synthesis of scaffold monomers or polymers. Some scaffold polymers for imprinting are with crosslinking elements, the imprinted polymer network can directly form films and membranes, which is benefit to the further application.

In conclusion, molecular imprinting is a promising technology for protein recognition, various approaches of protein imprinting have been developed, each of which may contain several imprinting strategies such as bulk imprinting, surface imprinting, epitope imprinting and scaffold imprinting. With the help of the development of polymerization technique, biological technique, computer modulation and so on, more and more novel imprinting methods will be innovated to meet the requirement of the tailor made imprinting of proteins. 


\subsection{Polymerization}

\subsubsection{Controlled/living radical polymerization}

As the development of polymer materials, controlled radical polymerization was developed from conventional radical polymerization integrating the living polymerization technique with tailor made structure and functionality. The living polymerization was first found by Szwarc by anionic polymerization of styrene in $1956 .{ }^{[43]}$ The transfer and termination reactions were avoided in a high vacuum reaction environment to minimize the impurities and oxygen in a nonpolar solution. The key issue of living ionic polymerization is the equilibrium between growing polymer chain (active species) and dormant species, which has been investigated in details by cationic vinyl polymerization in 1990 s. $^{[4]}$ The inherent disadvantages such as small range of monomers and low tolerance to the environment limited the development of living ionic polymerization. The radical polymerization can overcome such limitations, which has the largest range of monomers. The radical itself is tolerant to many functionalities, including acidic, hydroxyl and amino groups. The radical polymerization has been the key synthesis route for polymerization industry. Radical polymerization comprises four elementary steps: initiation, propagation, termination and transfer. ${ }^{[45]}$

$$
\begin{aligned}
& \text { Initiation } \\
& \qquad \begin{array}{l}
\mathrm{I}-\mathrm{I} \stackrel{\mathrm{k}_{\mathrm{d}}}{\longrightarrow} 2 \mathrm{l} \\
2 \mathrm{H}+\mathrm{M} \stackrel{\mathrm{k}_{\mathrm{i}}}{\longrightarrow} \mathrm{P}_{1} .
\end{array}
\end{aligned}
$$

Propagation

$$
\mathrm{P}_{1} \cdot \mathrm{M} \stackrel{\mathrm{k}_{\mathrm{p}}}{\longrightarrow} \mathrm{P}_{\mathrm{n}+1}
$$

Termination

$$
\begin{aligned}
& P_{n} \cdot+P_{m} \cdot \stackrel{k_{t c}}{\longrightarrow} P_{n+m} \\
& P_{n} \cdot+P_{m} \cdot \stackrel{k_{t d}}{\longrightarrow} P_{n}=+P_{m}^{H}
\end{aligned}
$$

Transfer

$$
\begin{aligned}
& P_{n^{\bullet}}+M \stackrel{k_{\operatorname{trM}}}{\longrightarrow} P_{n}+P_{1} \cdot \\
& P_{n} \cdot+P_{X} \stackrel{k_{\operatorname{trP}}}{\longrightarrow} P_{n}+P_{X} \cdot \\
& \left\{\begin{array}{r}
P_{n^{\bullet}}+T A \stackrel{k_{\operatorname{trTA}}}{\longrightarrow} P_{n}-A+T \cdot \\
T \cdot+M \stackrel{k_{\operatorname{trTA}}}{\longrightarrow} P_{1} \cdot
\end{array}\right.
\end{aligned}
$$

Scheme 2.1 Mechanism of radical polymerization. 
Polymer chain transfer can be avoided by applying appropriate conditions in conventional radical polymerization, but it is difficult to minimize the combination of bimolecular radicals, which is diffusion controlled. Because of the high rate of termination, the life of propagation radical is too short for any synthetic manipulation, end functionalization, or addition of a second monomer to make a block copolymer. The controlled radical polymerization minimizes the chain termination by intermittent form of the propagation radical. The dynamic equilibrium between propagating radical and dormant species is the key issue of controlled radical polymerization. This technique is also considered as living polymerization and comprises of two approaches. First approach is used by nitroxide-mediated polymerization (NMP) and ATRP, which relies on the persistent radical effect. The newly generated radicals are rapidly trapped in a deactivation process by species $\mathrm{X}$ which is nitroxide (NMP) or organometallics species (ATRP) to form a dormant species before bimolecular combination. The persistent species $X$ can only cross-couple with the propagating radical not to reach other. The dormant species can be activated reversibly to form a propagating radical again and leads to reduce the termination possibility. The life of the propagating radical is extended from seconds to more the one day, this essentially gives rise to control the polymerization. ${ }^{[45]}$

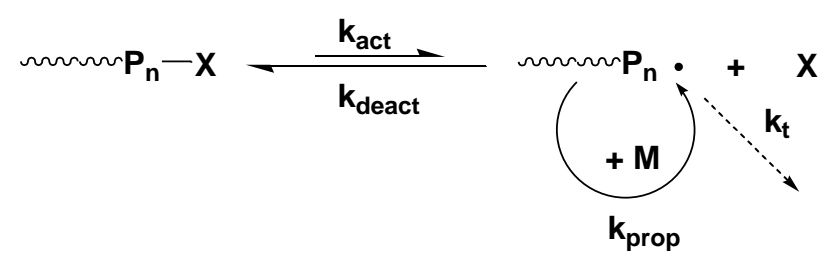

Scheme 2.2 Mechanism of radical activation/deactivation process by NMP and ATRP.

Another approach bases on degenerative transfer by using transfer agent (RAFT) which has much higher concentration than that of the propagating radical and thus shift the polymerization reaction to dormant species side not the termination direction. ${ }^{[45]}$

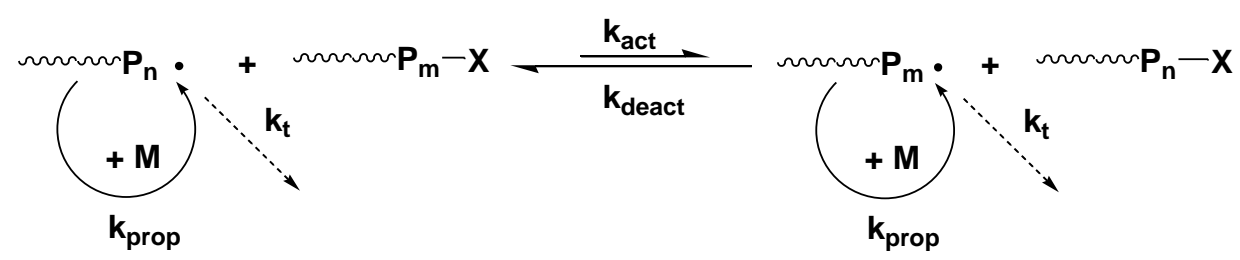

Scheme 2.3 Mechanism of radical transfer by RAFT. 


\subsubsection{Nitroxide-mediated polymerization (NMP)}

The polymerization control of NMP is determined by the dynamic equilibration between dormant alkoxyamine and propagating radical. The homolytic cleavage of a relatively weak $\mathrm{C}-\mathrm{O}$ bond generates a growing racial and a less reactive nitroxide radical which plays a mediator (persistent radical) role and can only couple with the propagating radical to form the dormant species and not with themselves or reacts with monomer. ${ }^{[46]}$

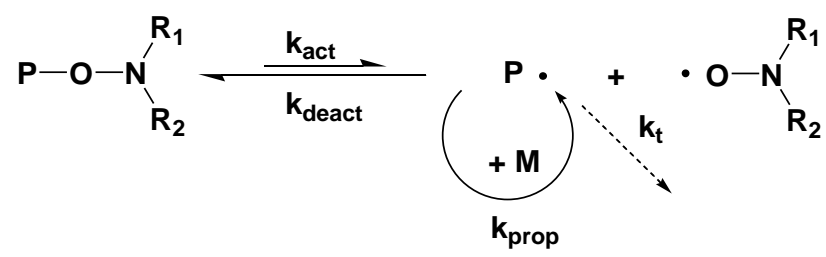

Scheme 2.4 Mechanism of NMP by TEMPO initiation.

2,2,6,6-Tetramethyl-1-piperidynyl-N-oxyl (TEMPO) and its derivatives are the typical mediators for NMP. The covalent C-O bond of alkoxyamine (TEMPO) is relatively stable and most of this kind polymerizations were carried out in a high temperature $\left(120{ }^{\circ} \mathrm{C}\right.$ by Georges), ${ }^{[47]}$ the TEMPO based NMP was limited by the range of monomers such as acrylates and methacrylates. ${ }^{[44]}$ Numerous derivatives of nitroxide, such as DEPN, ${ }^{[48]}$ TIPNO $^{[49]}$ and TEMPO-TMS, ${ }^{[50]}$ were synthesized to reduce the $\mathrm{C}-\mathrm{O}$ bond dissociation energy and extend the monomer range. The steric effect of these bulk decrease the bond dissociation energy of the aklkoxyamine efficiently and the NMP of acrylates can be carried out at $70^{\circ} \mathrm{C} .{ }^{[45]}$

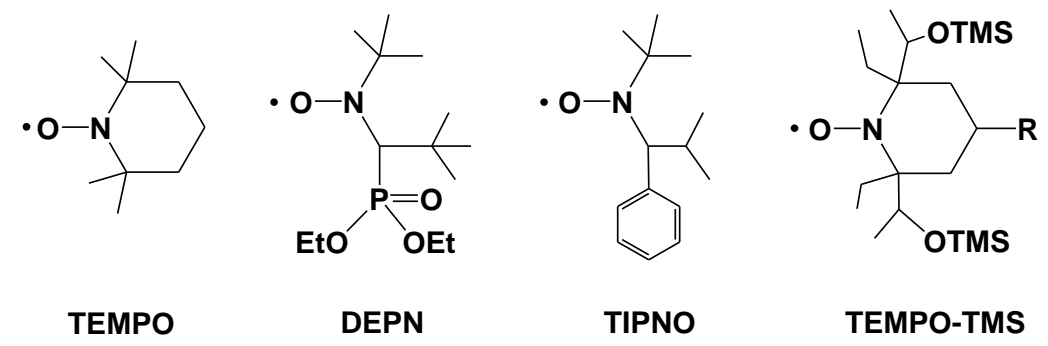

Scheme 2.5 Four examples of nitroxides employed by NMP.

\subsubsection{Reversible addition-fragmentation chain transfer (RAFT)}

The success of RAFT polymerization is based on a sequence of addition-fragmentation equilibrium as shown below (Scheme 2.6). 


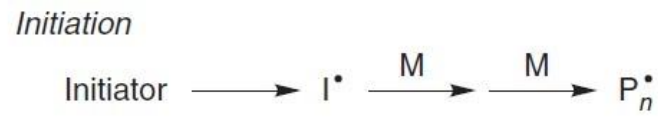

Reversible chain transfer

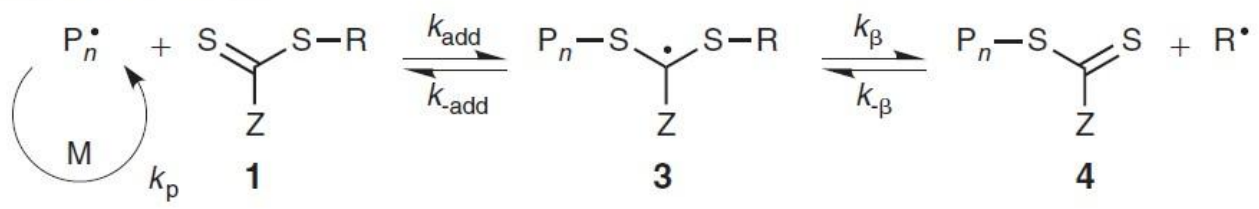

Reinitiation

$$
\mathrm{R}^{\cdot} \stackrel{\mathrm{M}}{\underset{k_{\mathrm{i}}}{\longrightarrow}} \mathrm{R}-\mathrm{M}^{\cdot} \stackrel{\mathrm{M}}{\longrightarrow} \stackrel{\mathrm{M}}{\longrightarrow} \mathrm{P}_{m}^{\cdot}
$$

Chain equilibration

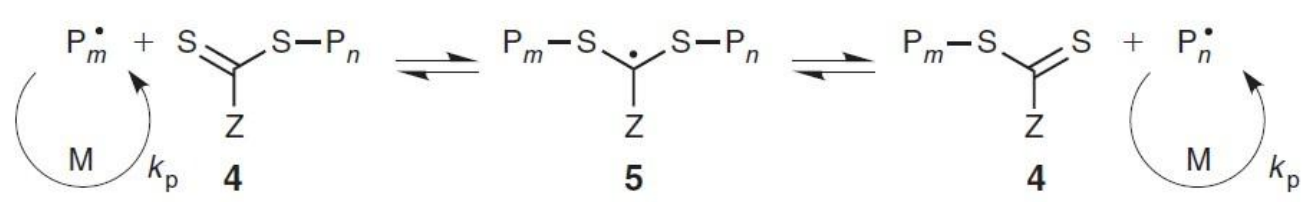

Termination

$$
\mathrm{P}_{n}^{\cdot}+\mathrm{P}_{m}^{\cdot} \stackrel{k_{\mathrm{t}}}{\longrightarrow} \text { Dead polymer }
$$

Scheme 2.6 Mechanism of RAFT with a thiocarbonylthio transfer agent. ${ }^{[51]}$

The initiation and termination is similar to the conventional radical polymerization. After initiation, the initial radical adds to the monomers to form the propagating radical ( $\left.k_{\text {add }}\right)$, then propagating radical adds to the transfer agent (typically thiocarbonylthio compound $\mathrm{RSC}(\mathrm{Z})=\mathrm{S})$ to form a polymeric thicarbonylthio compound $\mathrm{P}_{\mathrm{n}} \mathrm{SC} \cdot(\mathrm{Z})=\mathrm{S}$ and a new radical $\mathrm{R}^{\cdot}$ through fragmentation (reversible chain transfer, $k_{\beta}$ ). The radical $R^{\cdot}$ adds to the monomers to form new propagating radical $\mathrm{P}_{\mathrm{m}}$ (reinitiation, $\mathrm{k}_{\mathrm{i}}$ ). Rapid equilibrium between the propagating radical $\mathrm{P}_{n}{ }^{\cdot}$ or $\mathrm{P}_{\mathrm{m}}{ }^{\cdot}$ and the dormant species $\mathrm{P}_{\mathrm{n} / \mathrm{m}} \mathrm{SC} \cdot(\mathrm{Z})=\mathrm{S}$ to avoid their termination which results in a long life time of the propagating radicals and equal chance for all chains to grow. Therefore the control of the polymerization is improved with a narrow polydispersity.

The controlled/living mechanism of RAFT polymerization based on the degenerative transfer is different to the polymerization controls by NMP and ATRP which involve reversible deactivation of propagating radicals by bimolecular radical combination (NMP) or atom transfer (ATRP). The dormant species (the alkoxyamine in NMP or the halo-compound in 
ATRP) can be later activated to form the propagating radicals. The position of the deactivation-activation equilibrium and the persistent radical effect determine the rate of polymerizations by NMP and ATRP. In RAFT polymerization, the deactivation-activation equilibrium is chain-transfer reactions. After initiation from external initiator, the amount of radical was kept constant during the propagation, neither formed nor destroyed. The transfer agents play a key role in the RAFT polymerization which inclusive xanthates, dithioethers, DTC and trithiocarbonate. The structure of the transfer agent has a strong influence on the RAFT polymerization. For most applied RAFT agent dithioester, the freeracial leaving $R$ and substituted $Z$ groups determine the control of the polymerization.

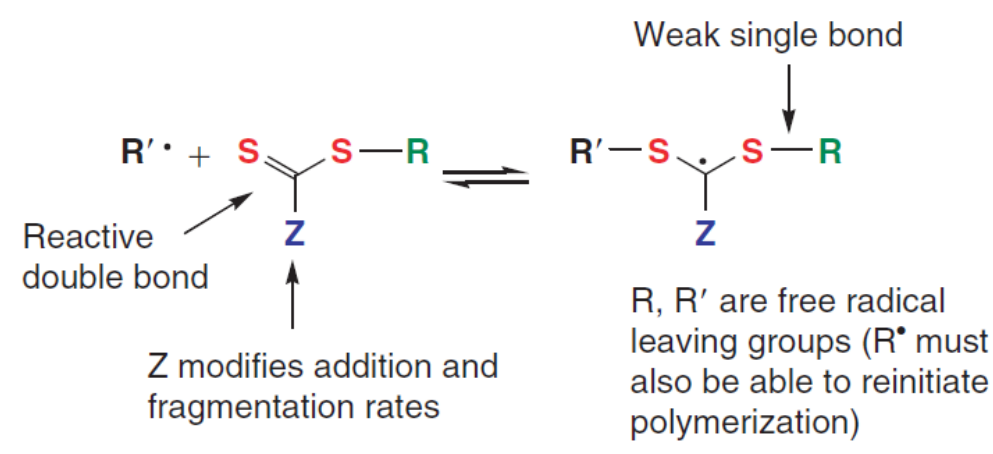

Scheme 2.7 Structural features of thiocarbonylthio RAFT agent and the intermediate formed on radical addition. ${ }^{[51]}$

$\mathrm{R}$ with steric hindrance and electron-withdrawing group is benefit to a rapid fragmentation. The $\mathrm{R}^{\cdot}$ should be more stable than $\mathrm{P}_{\mathrm{n}} \cdot$ to initiate the polymerization. ${ }^{[52]}$ The stabile $\mathrm{Z}$ group leads to efficient polymerization for styrene, methacrylate, and the $Z$ group with less stability such as $-\mathrm{OR}$ and $-\mathrm{NR}_{2}$ is benefit to improve the control on the polymerization of vinyl esters. $^{[53]}$<smiles>[Z2][R]([H])([H])SCC</smiles>

Scheme 2.8 For $\mathrm{R}$, rates of fragmentation decrease from left to right. For $Z$, addition rates decrease and fragmentation rate increase from left to right. ${ }^{[51]}$ 


\subsubsection{Atom transfer radical polymerization (ATRP)}

Since 1995, Matyjaszewski and his coworkers innovated the ATRP, ${ }^{[54]}$ which has been the most extensively investigated and widely applied controlled/living radical polymerization technique. The significant advantages are commercial availability of all the necessary ATRP reagents, such as transition metal compounds, ligands, alkyl halide initiators, and large range of monomers. The synthesized polymer can be precisely controlled in both structure and functionality. In ATRP, alkyl halide initiators or dormant species ( $R X$ or $P_{n} X$ ) is reversibly activated via cleavage of the carbon-halogen bond by low-oxidation-state metal complex $M t^{2} L_{m}$ (activator) to form both propagating radicals $\left(R^{*} / P_{n}{ }^{\cdot}\right)$ and higher-oxidation-sate metal complex with coordinated halide and ligand $\mathrm{XMt}^{\mathrm{z}+1} \mathrm{~L}_{\mathrm{m}}$ (activation constant $\mathbf{k}_{\text {act }}$ ). $\mathrm{R} \cdot \mathrm{Pn} \cdot$ can then add to the monomer $M$ (propagating constant $\mathbf{k}_{\mathrm{p}}$ ), before it is deactivated by $\mathrm{XMt}^{2+1} \mathrm{~L}_{\mathrm{m}}$ to form a dormant species $\mathrm{P}_{\mathrm{n}}-\mathrm{X}$ (deactivation constant $\mathbf{k}_{\text {deact }}$ ) and terminated (termination constant $\mathbf{k}_{\mathbf{t}}$ ). The dormant species in ATRP equilibrium can be polymer chains able to grow in one or many directions, or polymers attached to functional particle, surface, biomolecule and so on (Figure 2.7). ${ }^{[13,45]}$

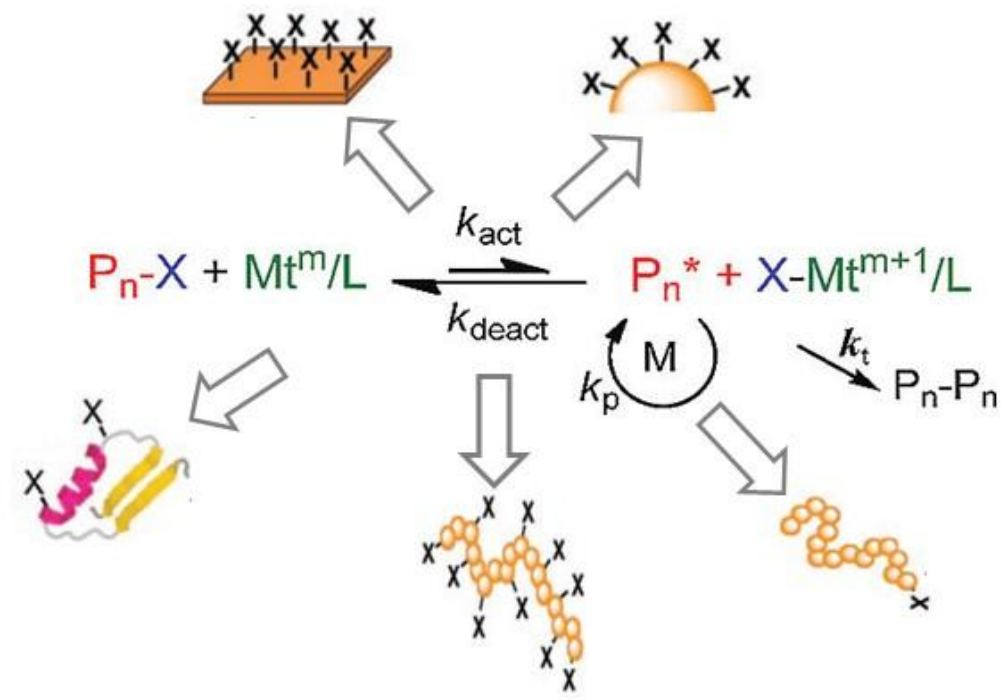

Figure 2.7 Mechanism of ATRP and the possibility of the substrates. ${ }^{[13]}$

The rate of ATRP $\left(R_{p}\right)$ can be calculated with the following equation:

$$
R_{\mathrm{p}}=\frac{k_{\text {act }}}{k_{\text {deact }}} k_{\mathrm{p}} \frac{[\mathrm{M}][\mathrm{RXX}]\left[\mathrm{Mt}^{z} \mathrm{~L}_{m}\right]}{\left[\mathrm{XMt}^{2+1} \mathrm{~L}_{m}\right]}=K_{\mathrm{ATRP}} k_{\mathrm{p}} \frac{[\mathrm{M}][\mathrm{RX}]\left[\mathrm{Mt}^{2} \mathrm{~L}_{m}\right]}{\left[\mathrm{XMt}^{2+1} \mathrm{~L}_{m}\right]}
$$


Where $\mathrm{K}_{\text {ATRP }}$ is the ATRP equilibrium constant (cf. Scheme 2.2). The polydispersity index $M_{w} / M_{n}$ can be calculated as below:

$$
\frac{M_{\mathrm{w}}}{M_{\mathrm{n}}}=1+\left[\frac{k_{\mathrm{p}}[\mathrm{RX}]_{0}}{k_{\text {deact }}\left[\mathrm{XM \textrm {Xt } ^ { + } + 1} \mathrm{L}_{m}\right]}\right]\left[\frac{2}{\mathrm{conv}}-1\right]
$$

The radical of ATRP is produced from the dormant species by homolytic dissociation from C$X$ via inner-sphere electron transfer (ISET) or outer-sphere electron transfer (OSET) (Figure 2.8). By ISET, halide atom is transferred through a $\mathrm{Cu}-\mathrm{X}-\mathrm{C}$ transition state via a single electron transfer. The OSET is multi stepwise via radical anion intermediated. Most ATRP based on copper catalyst carried out by the ISET to homolyticly dissociate the alkyl halide, because OSET has a higher energy barrier than ISET, which results from the slow electron transfer from catalyst to dormant species alkyl halide. ${ }^{[55]}$ Beside copper, $\mathrm{Ti}, \mathrm{Re}, \mathrm{Fe}, \mathrm{Mo}, \mathrm{Ru}, \mathrm{Rh}, \mathrm{Ni}$ and Pd were also used to form the catalyst complex by ATRP. ${ }^{[56]}$

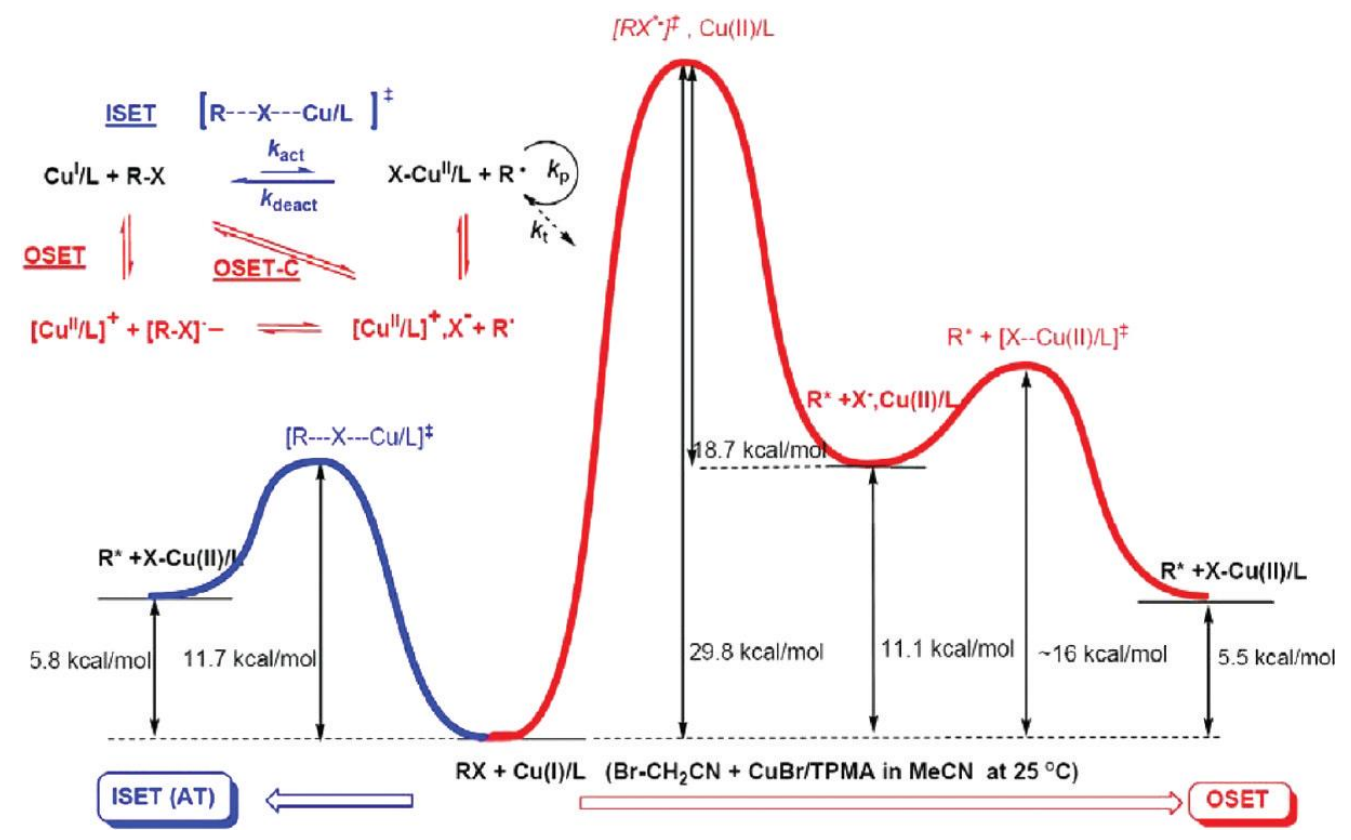

Figure 2.8 Comparison of the free energies during an ISET and OSET process for the reaction of bromoacetonitrile with Cul/TPMA catalyst in MeCN at $25^{\circ} \mathrm{C} .{ }^{[57]}$

The equilibrium between the propagating radical and the dormant species depends on the catalyst including metal and ligand, alkyl halide, monomer and medium. The reactivity of 
alkyl halide is determined by the alky group and the transferable halogen. For alky group, the subsequence of reactivity is $3^{\circ}>2^{\circ}>1^{\circ}$ and for halogen is $\mathrm{I} \mathrm{Br}>\mathrm{Cl}$, which are according to bond dissociation energy of alkyl halide, and the ability of the alkyl group to stabilize the radical is another impact factor to the reactivity of the initiator. Normally electron donor effect enhances the stability of radical such as cyano group, and phenyl group. ${ }^{[58]}$

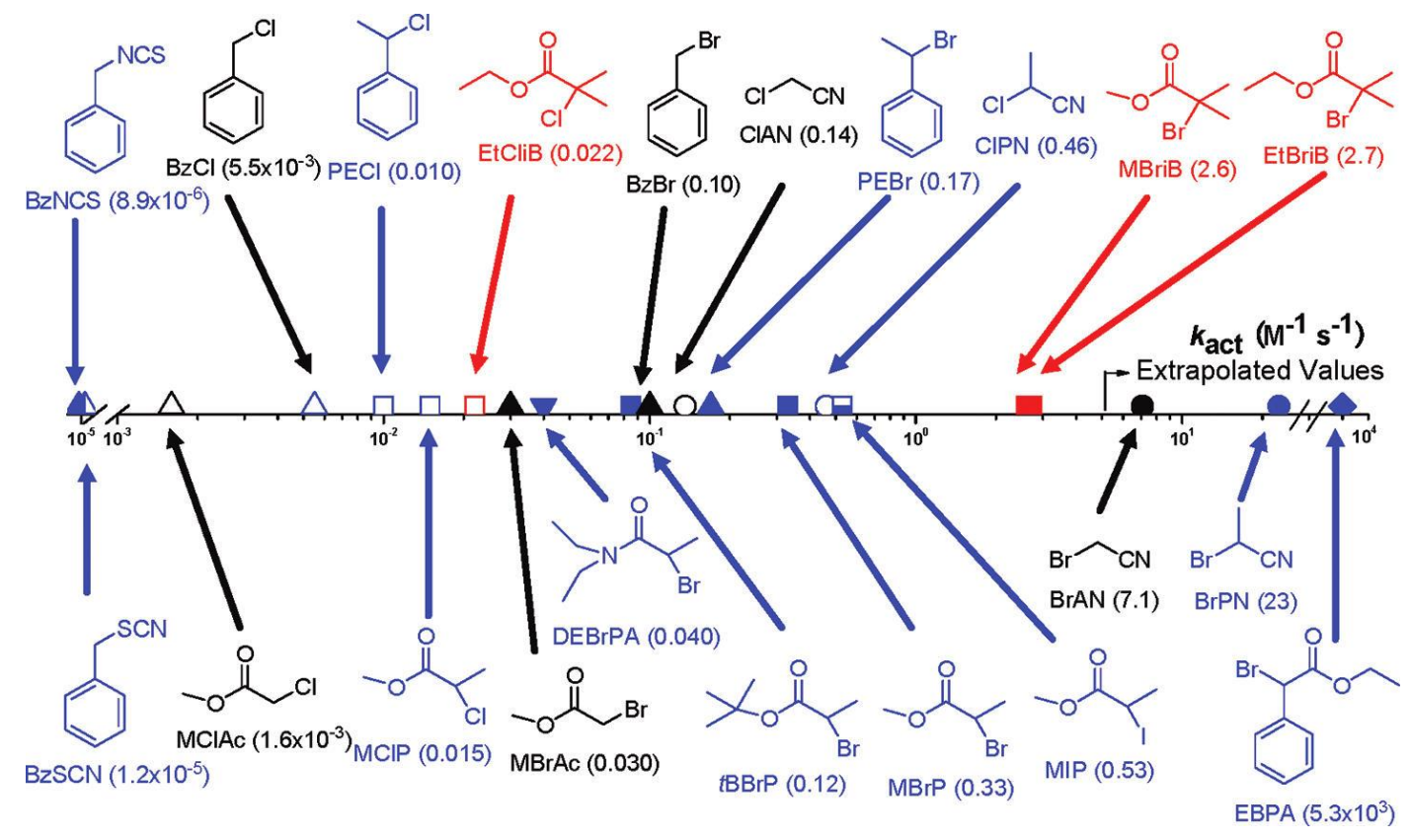

Figure 2.9 ATRP activation rate constants for various initiators with CuIX/PMDETA ( $\mathrm{X}=\mathrm{Br}$ or $\mathrm{Cl}$ ) in MeCN at $35^{\circ} \mathrm{C} .3^{\circ}$ : red; $2^{\circ}$ : blue; $1^{\circ}$ : black; isothiocyanate/thiocyanate: left half-filled; chloride: open; bromide: filled; iodide: bottom half-filled; amide: $\boldsymbol{\nabla}$; benzyl: $\boldsymbol{\Delta}$; ester: $\square$; nitrile: 0 ; phenyl ester: $\diamond$. ${ }^{[57]}$

The activity of the transition metal complex has a large range, the $\mathrm{Cu}$ complex activity depends on the ligands which serve to adjust the atom transfer equilibrium and to provide appropriate catalyst solubility with an order following: tetradentate (cyclic-bridge) > tetradentate $($ branched) $>$ tetradentate $($ cyclic) $>$ tridentate $>$ tetradentat (linear) $>$ bidentate. The nature of $\mathrm{N}$ atom also effects the activity of the $\mathrm{Cu}$ compex with a sequence: acryl amine $<$ acryl imide $<$ alkyl amine $\approx$ pyridine. ${ }^{[59]}$ The activity increases with increasing number of nitrogen coordination site with sequence $N 1<N 2<N 3<N 4$. The linking unit between $N$ atoms also influences the activity of catalyst complex with sequence: $\mathrm{C} 2>\mathrm{C} 3>\mathrm{C} 4{ }^{[60]}$ 


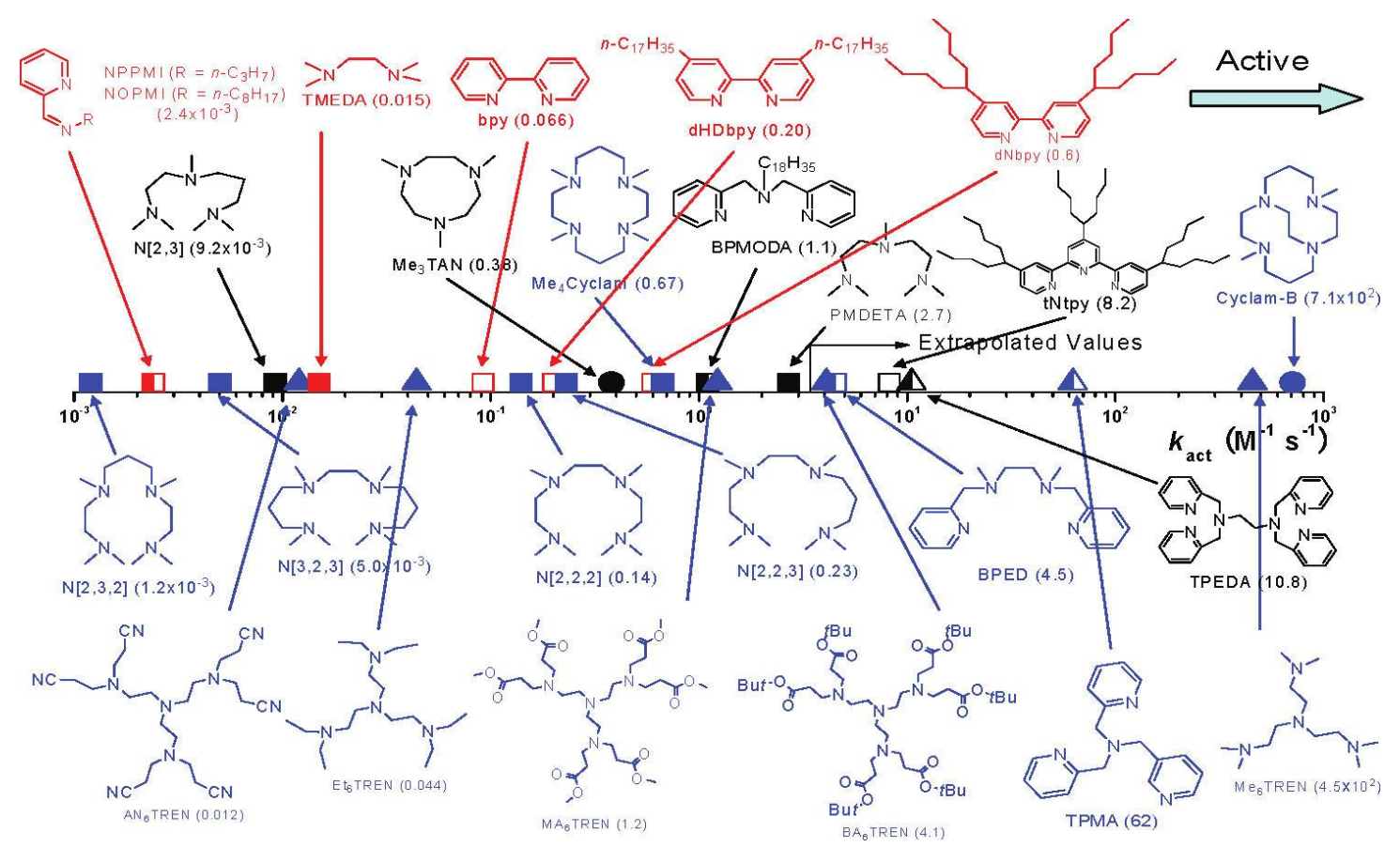

Figure 2.10 ATRP activation rate constants for various ligands with EtBriB in the presence of CulBr in $\mathrm{MeCN}$ at $35{ }^{\circ} \mathrm{C}$. N2: red; N3: black; N4: blue; amine/imine: solid; pyridine: open; mixed: left-half solid; linear: $\square$; branched: $\boldsymbol{\Delta}$; cyclic: . $^{[57]}$

ATRP is tolerant to various functional groups like conventional radical polymerization such as hydroxyl, amino, amindo, ether, ester, epoxy, siloxy and which leads to a large range of monomers including styrenes, methacrylates, acrylates, acrylamides, vinyl pyridines, acrylonitriles, vinyl acetate, vinyl chlorides and so on. The equilibrium constant is inferred by monomer, choice of the combination of initiation and catalyst is important to process successfully polymerization for different monomers. The reactivity of monomers can be ordered as the sequence: acrylonitrile $>$ methacrylates $>$ stryren $\approx$ acrylates $>$ acrylamides $>$ vinyl chloride $>$ vinyl acetate. ${ }^{[45]}$

ATRP is usually carried out in a polar solvent which is benefit to form a rapid equilibrium between propagating radical and dormant specie via stabilization of the anion $\mathrm{Cu}(\mathrm{II})$ complex than the neutral $\mathrm{Cu}(\mathrm{I})$ complex. Some ATRP even process in water. Temperature and pressure has the similar effect on ATRP like conventional radical polymerization. High temperature and pressure can accelerate ATRP rate. ${ }^{[61]}$

ATRP has been proved as a tailor made technique to prepare polymers with controlled polymer architecture such as topology (linear, star, cyclic, comb, brushes, networks, dendritic, and hyperbranched), composition (statistical, blocks, stereoblocks, multi block 
copolymers, multi segmented block copolymers, graft, periodic, alternating, and gradient copolymers), and functionalities (incorporated into side chains, end group(homo- and heterotelcjelic), and in many arms of stars and hyperbranched materials). ${ }^{[13]}$

a. Topology

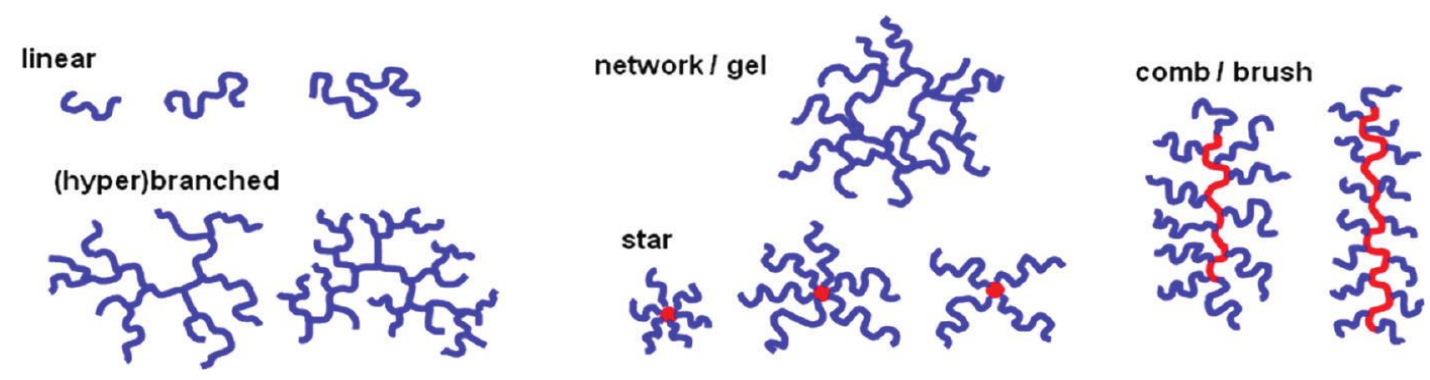

b. Composition/microstructure

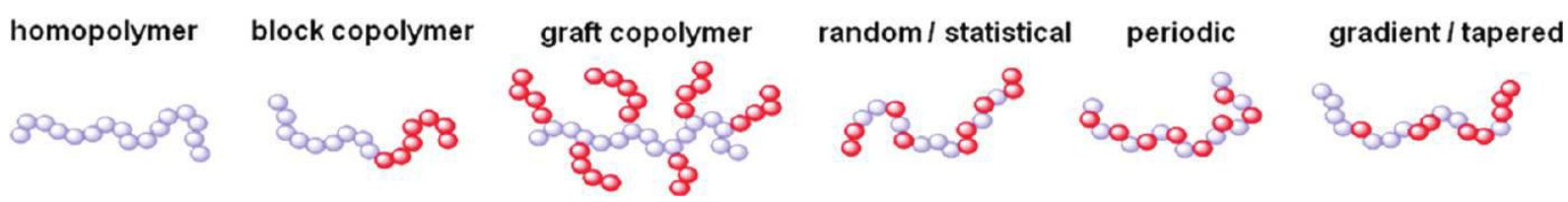

c. Functionality

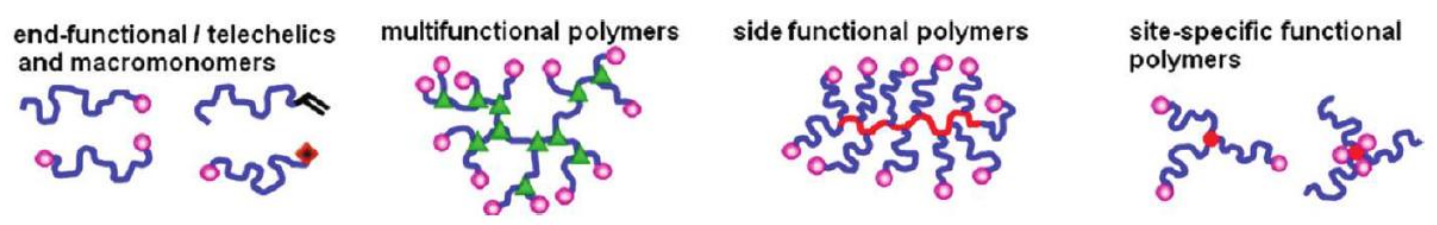

Figure 2.11 Examples of controlled macromolecular architecture in polymers prepared by ATRP, a: controlled composition/microstructure; b: controlled topology; c: controlled functionality. ${ }^{[13]}$

\subsubsection{Surface grafting}

Irrespective the fact that mechanical property of material is decided by its bulk phase, many applications of material are determined by the surface property. Manipulation of surface property via polymer grafting has a long history and nowadays attracted more and more interests. ${ }^{[62]}$ Surface grafting consists of physical grafting and chemical grafting. The physical grafting is based on the physical interaction to immobilize the polymer brush on the substrate surface, such kind of grafting is not stable by application in harsh condition. Stable chemical grafting includes "grafting to" and "grafting from" approaches. The "grafting to" approach depends on the combination of the polymer with end functional group and the surface reactive site. The grafted polymer can be controlled precisely with tailor made 
property, however, the low density of the grafted polymer limits the application of such method. The tethered polymer chain with steric hindrance prevents subsequent polymer grafting and reduces the surface coverage. The grafting from approach by initiation of the surface bound initiator is an alternative to improve the grafted polymer density via monomer addition instead of the polymer chain "addition". ${ }^{[63]}$

Surface grafting via conversional radical polymerization such as electrons, UV or plasma treatment has the inherent limitation of the poor control on the grafted polymer functionality and architecture, the limitations can be overcome by implement of the living radical polymerization to surface grafting technique. Surface initiated ATRP has been proved one of the most powerful methods to modify the substrate surface with control over brush length, density, polydispersity and composition. ${ }^{[13,64]}$

For the substrate self with ATRP initiator such as poly(4-vinylbenzyl chloride) (PVBC), the ATRP can directly carried out on the surface, otherwise immobilization of ATRP initiator is the first step of grafting approach. The fluoride group on the surface can be converted to chloride or bromide directly. Surface with hydroxyl or amino groups can also directly react with the initiator inclusive reagents such as 2-bromoisbutyryl bromide and 4(chloromethyl)benzoyl chloride (with the help of triethylamine (TEA)) to form bromide or chloride initiators. The strategy for initiator immobilization on the surface without hydroxyl group is to convert the surface group to hydroxyl group and subsequent immobilization of ATRP initiator. The resin surface group of aldehyde can be converted to hydroxyl group via amino, ring-opening of $\delta$-gluconalacton. The ATRP initiator can be immobilized in Nylon membrane by the activation with formaldehyde. PP surface $\left(\mathrm{CH}_{2}\right)$ was activated for ATRP via the UV-initiation of benzophenoyl 2-bromosibutylrate. Polyimide film has been anchored with benzyl chloride initiating sites for SI-ATRP by chloromethylation with paraformaldehyde/ $\mathrm{Me}_{3} \mathrm{SiCl}$ in presence of $\mathrm{SnCl}_{4}$. The poly(vinylidene fluoride) (PVDF) was immobilized with bromide through the thermal reaction of PVDF and 2-(2bromoisbutyl)ethyl acrylate. Chemical vapor deposition of bromide was used to graft poly(dimethylsiloxane) (PDMS), poly(methyl methacrylate) (PMMA), and polystyrene (PS) via SI-ATRP. ${ }^{[64]}$ 


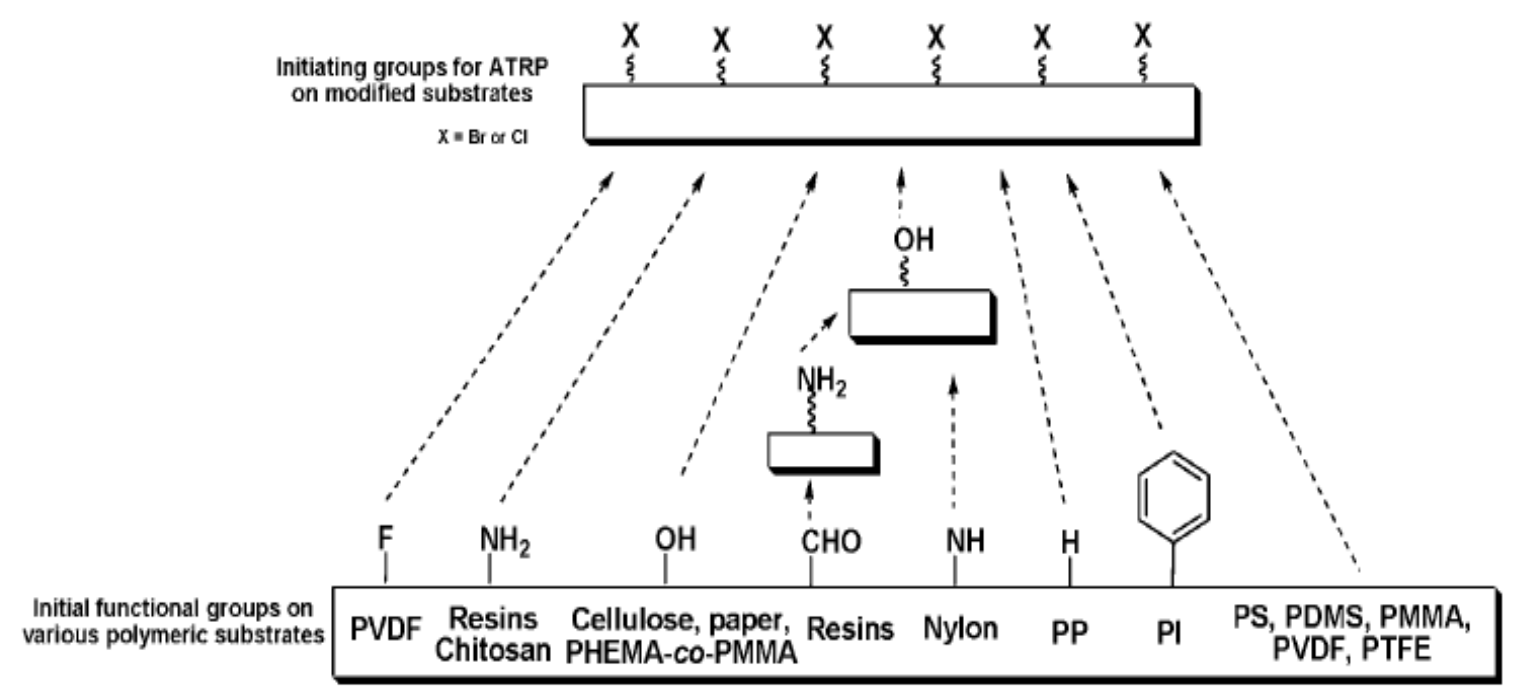

Figure 2.12 Immobilization of ATRP initiators on organic/polymeric substrates. ${ }^{\text {[64] }}$

The ATRP initiator immobilization on inorganic surface normally with spacer between the surface and initiating sites (for example bromoester, bromoamide, chloromethylpenyl, and sulfonylchloride) engages the reaction of silanization and gold-thiol interaction. The silanization of the surface of oxidized titanium, glass, silicon, silica and magnetic nanoparticle (FexOy) with hydroxyl group introduce spacer and initiating groups or functional groups such as hydroxyl, amino, and double bound groups for subsequent immobilization of initiating sites. The strong bond of Au-S is used for the ATRP initiator immobilization by using alkane thiols and disulfides. Other investigations for the ATRP initiator immobilization include chemical vapor deposition polymerization (silicon, stainless steel, and glass), electrochemical reduction (diamond), electrografting (carbon fiber, stainless steel), and radical introduction of initiator $(\mathrm{Si}-\mathrm{H}){ }^{[64]}$ 


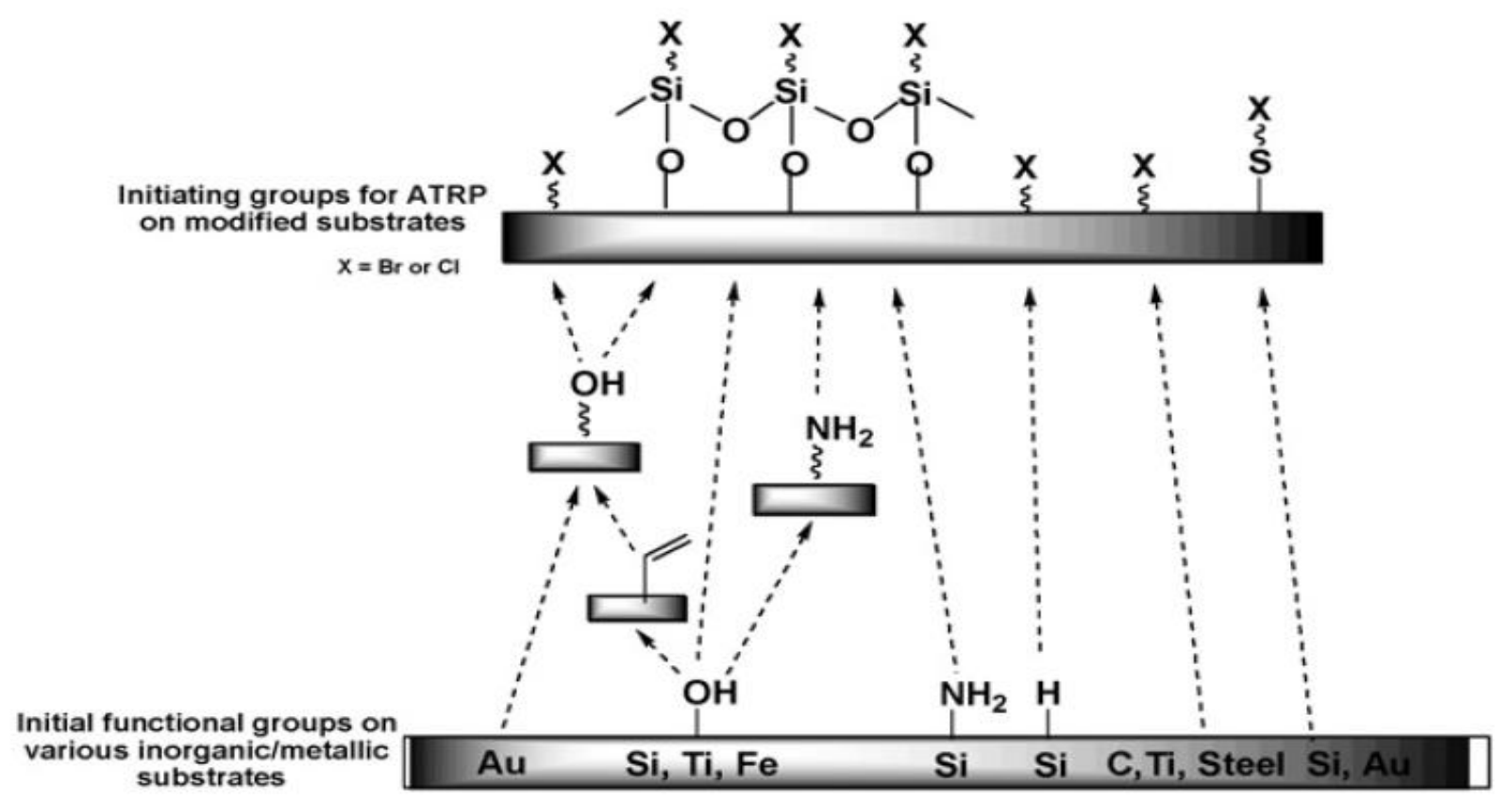

Figure 2.13 Immobilization of ATRP initiators on inorganic and metallic substrates. ${ }^{[64]}$

Smart polymer PNIPAAm was grafted on TE PET membrane and glass surface via SI-ATRP, the responsive abilities to temperature and $\mathrm{pH}$ were characterized successfully by Ulbricht and his coworkers. ${ }^{[11,65-66]}$ Poly((dimethylamino)ethyl methacrylate) (PDMAEMA) grafted PET membrane surface was obtained with similar SI-ATRP method, the responsive ability to temperature and $\mathrm{pH}$ was investigated by Tomicki in the same group. ${ }^{[14]}$ PDMAEMA grafted membrane via similar SI-ATRP method showed an ability to adsorb BSA via electrostatic interaction in aqueous puffer. ${ }^{[67-68]}$ Biocidal surface was obtained by SI-ATRP of PDMAEMA and subsequent quaternization with acryl halide on magnetic nanoparticle ${ }^{[69]}$ or viologen on stainless steel. ${ }^{[70]}$

Polyelectrolytes of PAA and PMAA responsive to $\mathrm{pH}$ and ionic strength are normally synthesized via ATRP of tert-butyl (meth)acrylate and subsequent hydrolysis ${ }^{\text {[1-72] }}$ or sodium(meth)acrylate. ${ }^{[73-74]}$ The side carboxyl group PMAA of PAA was used to immobilization protein on surface via electrostatic interaction or covalent ester coupling with the amino group of protein with the help of $\mathrm{N}$-hydroxysuccinimide (NHS) and $\mathrm{N}$-(3dimethylaminopropyl)- $N$ '-ethylcarbodiimide hydrochloride (EDC). ${ }^{[75]}$

Antifouling functional polymers such as poly(poly(ethylene glycol) methacrylate) $(\text { PPEGMA })^{[76]}$ and poly(oligo(ethylene glycol) methacrylate) $\left(\right.$ POEGMA) ${ }^{[77]}$ were grafted successfully on material surface via ATRP. Poly(2-hydroxlyethyl methacrylate) (PHEMA) 
showed protein repellency due to high hydrophilic property via SI-ATRP on PVDF membrane surface ${ }^{[78]}$ Zwitter ionic polymers with a mixture of anionic and cationic groups to form a hydration layer prevent protein adsorption and cell adhesion. Poly(methacryloyloxyethyl phosphorylcholine) (PMPC), ${ }^{[79]}$ poly(carboxybetaine methyacrylate) (PCBMA), ${ }^{[80]}$ and poly(sulfobetaine methacrylate) (SBMA) on PVDF membrane ${ }^{[81]}$ were grafted via SI-ATRP. 


\subsection{Membrane adsorber}

Membrane technology is considered as green technique with low energy consumption and has been established as one of the most successful processes in industrial applications for food, biotechnology, pharmacy and so on. The advantages of membrane technology are the relative ease of scale-up and integration into other processes. ${ }^{[82-84]}$ The greatest interest of membrane technology is applied in filtration processes of reverse osmosis (RO), nanofiltration (NF), ultrafiltration (UF) and microfiltration (MF) ${ }^{\left[{ }^{[5]}\right.}$ Except the size exclusion effect, the interactions between membrane and substances of interest mainly consist from ionic bond, hydrogen bond, hydrophilic/hydrophobic interaction and van der Waals force. Those are the key issue, which depend on the preparation of the membrane from special functional polymer or using functional additives during membrane formation. An important alternative of membrane functionalization which has attracted more and more attention is immobilization of functional ligands on the substrate membrane surface. ${ }^{[82]}$

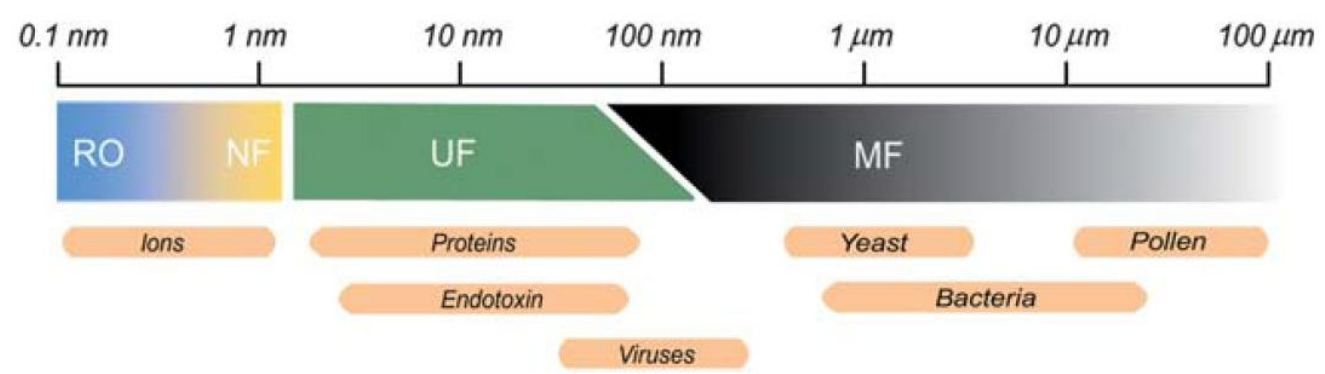

Figure 2.14 Schematic illustration of different pressure-drive membrane processed according to size of pores in the selective barrier and size of exemplaric targets for separation. ${ }^{\left[{ }^{86]}\right.}$

\subsubsection{Geometry of membrane adsorbers}

Membrane adsorbers are porous membranes, usually similar to MF membranes. The effective separation is based on reversible binding of target to the functionalized pore walls, this process is facilitated by convective flow through the pores. Membrane adsorbers can be classified generally into flat sheet, hollow fiber and spiral wound according to geometry. Usually, stacks of several flat sheet membranes which are mounted in holders are used instead of the single flat sheets in order to improve the performance of membrane adsorbers. For single flat sheet membrane adsorber, the low capacity to the interest is the most obvious disadvantage. Stacks of several membrane sheets can provide more adsorbent 
volume and increase the capacity, additionally, this model overcome the most limitations of single flat sheet membrane resulted from pore size distribution and small thickness. Stacks with large number of membranes reduce the flow dispersion and lead to a sharp breakthrough curve by chromatography, therefore increase the binding efficiency. Membrane stack is the most widely used material of the three type geometries, the mass transport has been intensively investigated by in theory and experiment. ${ }^{\text {[8] }}$

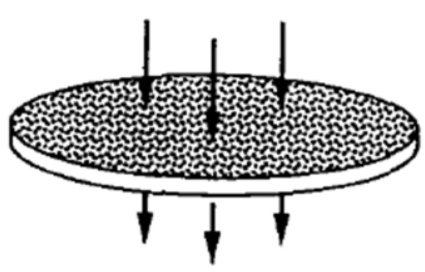

Single membrane sheet

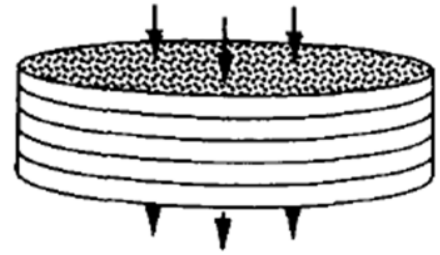

Membrane stack

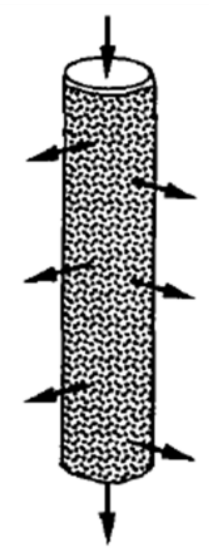

Hollow fiber

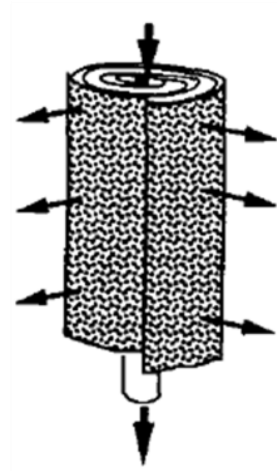

Spiral wound

Figure 2.15 Schematic presentation of different membrane adsorber geometries. ${ }^{\text {[84] }}$

Radial flow membrane adsorber prepared by spiral wound of flat sheet membrane round over a porous cylinder is also an alternative to improve the capacity of single flat sheet membrane adsorber. But the flow distribution by radial flow membrane adsorber is much more complicated than stack membrane adsorber resulted from the drop in superficial velocity of the liquid through the spiral wound membrane. However, radial flow membrane adsorber with high binding capacity still demonstrated a good potential in the industry.

A hollow fiber membrane has tubular geometry with the tubes typically ranging from 0.25 to $2.5 \mathrm{~mm}$ in diameter. Typical fiber membrane adsorber usually consists of a bundle of several hundred fibers which are potted together within a module in a shell and tube heatexchanger-type configuration. The direction of the liquid is parallel to the membrane surface and then gradually directed towards and through the pores driven by the hydrostatic pressure difference. The flow of hollow fiber membrane is more complicated than that of flat membrane by which the liquid is usually introduced directly to the membrane surface and penetrate through the membrane under outer pressure. Hollow fiber membrane provide large ratio of surface area to the liquid, the initial liquid flow will also acted as cross- 
flow leading to fouling reduction on the membrane surface. Due to the geometry, the breakthrough by hollow fiber membrane is much broader than that of flat sheet membrane and such module with short duration time is also a limit to be applied by pulse chromatography which needs a long processing time. ${ }^{[83]}$

\subsubsection{Chromatography with membrane adsorbers}

As the biotechnology and the pharmacy developed rapidly, the purification of biomacromolecules such as protein demands strongly a reliable, efficient methods for the purification of large amounts. ${ }^{[87]}$ Chromatography is by far the most widely used technique for protein analysis and separation. Traditional method by using packed bed has permanent limitations which are the high pressure drop, poor intra particle diffusion and large liquid volume. Membrane chromatography is an alternative to overcome these limitations. The convective flow of the solution through the membrane pores leads to small mass transfer resistance, thereby reduction of the process time and recovery liquid volume. Additionally, membrane adsorber is easier to scale-up than the packed bed module. ${ }^{[8]}$ An ideal membrane support for successful application in protein chromatography should possess the following properties. It should be micro/macroporous to preserve the protein mass transport and accommodate the free interaction of protein with ligand; prevent nonspecifical interaction between protein and the membrane support self; contain functional groups to allow activation by a wide variety of reactions; be chemically stable to withstand harsh conditions during adsorption, elution and regeneration, be physically stable to withstand transmembrane pressure, when applicable, sterilization by autoclaving, and readily available at low cost to facilitate industrial applications. The limitation for membrane support applications in chromatography lies generally in the necessity for chemically active sites in the membrane matrix for bonding the ligand. ${ }^{[89]}$

\subsubsection{Affinity chromatography}

The combination of membrane chromatography with affinity interaction provide affinity chromatography, which permits the purification of proteins based on mimicing biological functions rather than individual physical or chemical properties. The high specificity of affinity chromatography is due to the strong interaction between the attached biospecific 
ligand and protein of interest. ${ }^{[87,90]}$ IgG affinity membrane were mainly based on immobilized protein $A,^{[91-92]}$ protein $G^{[93-94]}$ and peptide ligand ${ }^{[92,95-96]}$ to purify the IgG by mimicking the natural antibody-antigen interaction. Metal ions such as $\mathrm{Cu}^{2+}, \mathrm{Ni}^{2+}$ and $\mathrm{Zn}^{2+}$ to bind the specific surface residues were immobilized on membrane surface to separate the protein mixtures. ${ }^{[97]}$ The low capacity of affinity chromatography is the significant disadvantage and persuades to develop other high capacity chromatography techniques such as ion-exchange chromatography and hydrophobic/reverse phase chromatography.

\subsubsection{Ion-exchange chromatography}

The major segment of membrane chromatography is ion-exchange membrane, which takes the advantage of electrostatic interaction between surface charges of protein such as amino acids and cluster of charged groups on membrane surface. Protein is adsorbed via displacement of counterions associated with the surface, discharging a complementary buffer salt in the process. Typically, when the $\mathrm{pH}$ of the adsorption buffer is above its isoelectric point (pl), a protein will bind to a positively charged anion exchanger. Below its pl, a protein will bind to a negatively charged cation exchanger. An appropriate buffer is the key issue for the protein adsorption via ion exchange, salt in buffer should be adequate to protect native protein structure from changes in $\mathrm{pH}$ adjacent to exchange surface (Donnan effect) and $\mathrm{pH}$ effects induced by sorption. ${ }^{\left[{ }^{[4]}\right.}$

The electrostatic interaction is sensitive to the environment such as ion concentration, $\mathrm{pH}$, and temperature, this provides a potential to separate proteins by efficient gradient elution. ${ }^{[98-99]}$ Usually, a high salt concentration, and extreme $\mathrm{pH}$ value are engaged to elute the adsorbed proteins. A strong ion-exchanger has better tolerance to high salt concentration and broad range of $\mathrm{pH}$ than the weak ion-exchanger. The charge property of membrane depends on the preparation of the basis membrane with ionizationable groups or the post treatment with ionizationable surface structure. For protein separation, carboxyl acid, sulfonic acid and sulfopropyl are the most cation exchangers, amines such as diethaminoethyl and quaternary ammonium are the typical anion exchangers. ${ }^{[83]}$

Additionally, the zwitterionic ion-exchangers containing positive and negative charges in a close proximity exhibit potential for protein separation by ion-exchange chromatography instead of the standard typical anion and cation ion-exchangers. Control of the ratio of 
electrostatic attration/replulsion forces between analytes and ion-exchange groups provides potential to improve the selectivity in ion chromatography. This can result in the ability to utilize relatively dilute eluents, which is considered to increase detector sensitivity. ${ }^{[100-102]}$

\subsubsection{Hydrophobic interaction chromatography}

Hydrophobic interaction chromatography separates proteins according to difference in the surface hydrophobicity of proteins by a reversible interaction, van der Waals force between protein and the hydrophobic surface of chromatography medium. Protein has three dimensional structure stabilized by the intra-molecular interaction and the interaction with surrounding solvent (cf. Figure 2.3). In an aqueous solution (benefit for hydrophobic interaction), most hydrophobic residues are buried in the interior of proteins resulted from the thermodynamic compromise, however, there is still part of the hydrophobic residues which are exposed on the surface. In pure water, the hydrophobic interaction between protein and ligand is weak, addition of salt in the buffer can enhance this interaction. Reduction of ionic strength is a promising method for selective elution. As the ionic strength decreases, the protein with the lowest hydrophobic interaction begins to elute from the chromatography media.

Membranes engaged for hydrophobic interaction chromatography are composite of hydrophobic polymers ${ }^{[103]}$ or surface modified with hydrophobic ligands. ${ }^{[104]}$ Ghosh et al. developed hydrophobic interaction chromatography based on commercial PVDF membrane to separate $\mathrm{Lyz}^{[105-106]}$ and IgG. ${ }^{[107-109]}$ Another commercial phenyl membrane from Sartorius was investigated by Kuczewsk ${ }^{[110]}$ and Fraud ${ }^{[111]}$ for protein separation by hydrophobic interaction.

\subsubsection{Reverse phase chromatography}

Reverse phase chromatography with hydrophobic stationary phase is named from the traditional hydrophilic chromatography with a reverse hydrophilic stationary phase. It based also the hydrophobic interaction between analyte and chromatography medium. A polar mobile phase was engaged, the hydrophobic analyte in this mobile phase has tend to bind to the hydrophobic stationary phase. For the selective elution, the polarity of the mobile phase 
is reduced by mixing with non-polar (organic) solvent, the analyte has higher hydrophobicity which results in a stronger interaction between molecule and stationary phase, more ratio of organic solvent will be required to elute the analyte. The denaturation of the protein and the strong interaction are significant disadvantage in comparing with other chromatographies.

\subsubsection{Molecular imprinted composite membrane adsorbers}

Since the first molecular imprinted membrane was synthesized by Piletsky in 1990, more and more attention has been attracted to investigate molecular imprinted composite membrane, especially in membrane separation, affinity membrane chromatography and membrane sensor. The separation mechanisms and transport property of molecularly imprinted membranes were then reviewed by Ulbricht et al. in 1999. ${ }^{[112]}$ However, most investigation focused only on small organic molecule imprinted membrane until now. Extension of this technology for protein imprinting is still a challenging work, which has been discussed in section 2.2. The strategies to synthesize the molecular imprinted membrane consist of bulk polymerization, surface grafting and integration of imprinted sphere or particle into membrane. ${ }^{[113]}$

Bulk polymerization is an in situ method based on chemical crosslinking, dry phase separation and wet phase inversion. For the chemical crosslinking, the imprinting of protein is seldom, most of them focused on the small organic molecules. Shea developed an imprinted membrane with 9-ethyladenine as template, methacrylic acid as functional monomer and EDGMA as crosslinker, the crosslinking was thermally initiated by the AIBN in DMF. The recognition property of the membrane was characterized by comparison of adenosine and guanosine filtration. ${ }^{[114]}$ Piletsky imprinted atrazine in a poly methacrylic acid matrix based bulk polymerization via UV initiation of 1,1'-azobis-(cyclohexanecarbonitrile) $(A C B N)$, the imprinted membrane demonstrated a high capacity of atrazine than non imprinted membrane, the specific property was confirmed via the comparison of the adsorption of atrazine to similar molecules such as simazin, prometryn and metribuzin. ${ }^{[115]}$ By integration of electrochemical devices and molecular imprinting, Wu et al. developed a $\mathrm{BHb}$ imprinted polyacrylamide membrane which was prepared by cyclic voltammetry in an electrolytic solution which contains BHb, AAm, and MBAA. Free radicals were electrogenerated by the reduction of peroxydisulfate, so that the $\mathrm{BHb}$ imprinted 
polyacrylamide was formed only at the electrode surface. ${ }^{[116]}$ Dry phase separation with solvent evaporation was engaged by Lin et al. to develop the BSA and hemoglobin imprinted agarose gel membrane. ${ }^{[117]}$

Kobayashi is the pioneer of imprinting via phase inversion, the poly-(acrylonitrile-co-acrylic acid) was used to imprint theophyline, ${ }^{[118]}$ the property of the imprinted membrane was investigated through variation of coagulation temperature ${ }^{[119]}$ and acid segment of the polymer chain. ${ }^{[120]}$ Ulbricht and his coworkers developed a Rhodamine B imprinted cellulose acetate-sufonated polysulfone blend membrane via phase inversion technique, the permeability of the imprinted membrane was investigated through variation of blend content, pore forming agent polyethylene glycol and total polymer concentration. ${ }^{[121]}$ Wet phase inversion technique was also applied for the protein imprinting, $\alpha$-amylase was imprinted in a membrane based on poly(ethylene-co-vinyl alcohol) by Silvestri, the protein imprinted membrane was characterize $\alpha$-amylase adsorption of imprinted membrane with the non-imprinted membrane, albumin was used as the competitive protein for adsorption. ${ }^{[122]}$

Surface imprinting on membrane substrate is an important extension of the synthesis of imprinted membrane from bulk polymerization. Kobayashi imprinted theophyline via photopolymerization on a UV-reactive membrane poly(acrylonitrile-co-diethylamino dithiocarbamoylmethylstyrene) by using acrylic acid as functional monomer and MBAA as crosslinker, ${ }^{[123]}$ This strategy was used later by Chen to imprint protein Lyz. The comparison of the imprinted membrane and non-imprinted membrane was carried out through isotherm adsorption of Lyz. The recognition property of imprinted membrane was investigated through different protein adsorptions. ${ }^{[124]}$ Piletsky et al. developed a surface imprinting on non UV-reactive polypropylene membrane, the imprinted polymer layer on the substrate membrane was achieved though soaking the substrate in a mixture of functional monomer 2-acrylamido-2-methylpropansulfonic acid (AMPS), crosslinker MBAA, photoinitiator benzophenone and template desmetryme and subsequent photoinitiation. ${ }^{[125]}$ This method was later improved by Ulbricht and his coworkers by using $\alpha$-scission photoinitiation benzoin ether on the membrane surface, an imprinting effect could only be detected when this initiator had been coated to the surface, and not for the identical reaction mixture containing the dissolved benzoinether, therefore, the imprinted polymer layer was 
controlled well on the membrane surface not in the bulk mixture. ${ }^{[82,126]}$ Fukazawa and Ishihara developed a fibronectin imprinted polyethylene membrane via UV irradiation, the template protein at first form a complex with silica bead, then this protein-silica particle add to a mixture include poly(2-methacryloyloxyethyloxycarbonyl 4-phenylazido) with UVreactivity as scaffold polymer. After photoreaction, the protein and silica beads were detached from substrate by sonication and left imprinted structures on PE membrane surface. $^{[127]}$

Molecular imprinted composite membrane can also be prepared by integration with imprinted nanosphere or particle. Silvestri introduced two methods to integrate the imprinted nanosphere to membrane. First the nano imprinted sphere was directly immobilized on membrane surface by deposition and dry processes, this resulted in a stability problem for long time use. Second method is to mix the nano imprinted sphere into the polymer solution and entrapped inside the matrix during coagulation. ${ }^{[128-129]}$ A cryogel molecular imprinted composite membrane was synthesized by Asliyuce, anti-hepatitis B imprinted particle was first prepared, then introduced to a poly HEMA based polymer matrix by mixture and subsequent radical polymerization. ${ }^{[130]}$ 


\section{Chapter 3 Experiments}

\subsection{Materials}

Track-etched PET membranes with a pore diameter of about $675 \mathrm{~nm}$ (determined experimentally with gas flow / pore dewetting permporometry and scanning electron microscopy; cf. section 3.3.1 $)^{[10-11]}$ and a thickness of $23 \mu \mathrm{m}$ were purchased from Oxyphen GmbH (Dresden, Germany). Regenerated cellulose membranes (RC 60) with $75 \mu \mathrm{m}$ thickness, about $50 \mathrm{~mm}$ diameter, and $1.0 \mu \mathrm{m}$ average effective pore diameter were purchased from Whatman, Inc.

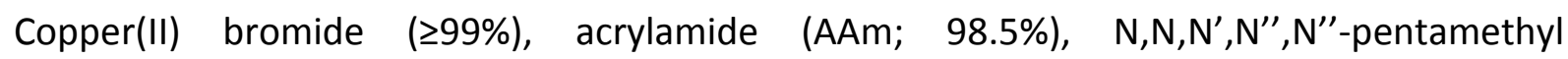
diethylenetriamine ( $\geq 99 \%$, PMDETA), N, $\mathrm{N}^{\prime}$-diisopropylcarbodiimide $(99 \%)$, and triethylamine (99\%) were purchased from Acros and used as received. Ethanolamine ( $\geq 99 \%), 4-\left(N, N^{\prime}-\right.$ dimethylamino)pyridine (DMAP; 98\%), sodium phosphate dibasic dodecahydrate ( $\geq 99 \%)$ lyzsozyme from chicken egg white (Lyz), and cytochrome $C$ from bovine heart (CyC) were from Fluka and used as received. Trifluoroacetic acid (TFA, >99.9\%) was purchased from Roth and used as received. N,N-dimethylaminopropyl acrylamide (DMPAA, $\geq 95 \%), 2-(\mathrm{N}, \mathrm{N}-$ dimethylamino)ethyl methacrylate (DMAEMA, $\geq 99 \%), \mathrm{N}$-(3-aminopropyl)methacrylamide hydrochloride (APMA. $\mathrm{HCl},>99 \%$ ) were obtained from Polysciences, Inc and used as received. Potassium phosphate (>99\%) and ethylenediaminetetraacetic acid disodium salt dihydrate (EDTA, >99\%) were obtained from AppliChem and used as received. Hydrochloric acid ( $\geq 37 \%)$ from Riedel-de-Haen, sulfuric acid ( $\geq 95 \%)$ from Merck, and natrium chloride $(99.9 \%)$ from VWR were used as received. $\alpha$-Bromisobutyrylbromide (BIB, 98\%), propionylbromide (97\%), 4-(chloromethyl)benzoyl chloride (>97\%), 2'2-bipyridine (Bpy; $299 \%$ ), copper(I) chloride (99.995\%), copper (II) chloride (99.999\%), N,N'-methylenebisacrylamide (MBAA; 99\%), and 1-hydroxybenzotriazole hydrate $(>97 \%)$, potassium permanganate $(\geq 99 \%)$, sodium diethyldithiocarbamate trihydrate $(>97 \%)$, tert-butylammomion bromide ( $\geq 98 \%)$, 2hydroxethelymethacarlate (97\%), albumin from human serum (HSA, $\geq 97 \%$, agarose gel lectrophoresis), $y$-globulin (IgG) from bovine blood ( $\geq 97 \%$, agarose gel electrophoresis), were obtained from Sigma-Aldrich and used as received. Tert-butylmethacrylate (tBMA; 98\%) was purchased also from Sigma-Aldrich and purified by distillation under argon atmosphere at 25 mbar pressure and $52{ }^{\circ} \mathrm{C}$ batch temperature. The BCA and the microBCA protein assay 
(Thermo Scientific, USA) and Micrococcus lysodeiktikus (ATCC No. 4698, Sigma-Aldrich) were used for Lyz concentration determination. Acetonitrile (MeCN), ethanol, methanol, tetrahydrofuran (THF), dichloromethane, and dimethylformamide (DMF) were of analytical grade, and water purified with the MilliQ system (Millipore) was used for all the experiments.

Tris[2-(dimethylamino)ethyl]amine ( $\mathrm{Me}_{6}$ TREN) was synthesized during according the work of Ciampolini $^{[131]}$ and Queffelec. ${ }^{[132]}$

\subsection{Syntheses}

\subsubsection{ATRP initiator immobilization on polyethylene terephthalate (PET) membrane}

The ATRP initiator (Al) immobilization was achieved via oxidative hydrolysis, activation, amination and intiatior immobilization (cf. Scheme 1.1). The membrane samples were all cut into $25 \mathrm{~mm}$ diameter disks, then washed and dried for turther using. Oxidative hydrolysis was carried out by immersion of PET base membranes (20 pieces) in a $100 \mathrm{~mL}$ reaction solution containing $5 \mathrm{~g} \mathrm{KMnO}_{4}$ and $0.375 \mathrm{~mol} / \mathrm{L} \mathrm{H}_{2} \mathrm{SO}_{4}$ and shaking with a rate of $200 \mathrm{rpm}$ for $2.5 \mathrm{~h}$ at ambient temperature. Membranes were then washed twice with water, treated four times for 5 min wuth $6 \mathrm{~mol} / \mathrm{L} \mathrm{HCL}$, washed four times with water and two times with ethanol and finally dried in overn at $50{ }^{\circ} \mathrm{C}$ overnight. For activation of carboxyl groups on membrane surface after oxidative hydrolysis, membranes were put in a $100 \mathrm{~mL}$ solution of $1.53 \mathrm{~g} \mathrm{~N}$ hydroxbenzotriazole and $0.63 \mathrm{~g} \mathrm{~N}, \mathrm{~N}^{\prime}$ - carbodiisopropydiimide in DMF shaking with a rate of $200 \mathrm{rpm}$ for 0 to $6 \mathrm{~h}$ at ambient temperature. After wash with DMF for 3 times, the membranes were put immediately in $0.5 \mathrm{~mol} / \mathrm{L}$ ethanolamine in DMF shaking with a rate of $200 \mathrm{rpm}$ for $3 \mathrm{~h}$ at ambient temperature. After amination, the samples were washed twice in DMF and twice in ethanol and dried in overn at $50{ }^{\circ} \mathrm{C}$ overnight. $\alpha$-bromoisobutyryl was immobilized on membrane surface via immersion in $5 \mathrm{~mL}$ solution containing $0.008 \mathrm{~mol} / \mathrm{L}$ $\alpha$-bromoisobutyryl bromide, $0.01 \mathrm{~mol} / \mathrm{L}$ triethamine, and $0.0005 \mathrm{~mol} / \mathrm{L} 4-\left(\mathrm{N}, \mathrm{N}^{\prime}-\right.$ dimethylamino)pyridine under the protection of argon in $25 \mathrm{~mL}$ vials with cap shaking with a rate of $200 \mathrm{rpm}$ for $3 \mathrm{~h}$ at ambient temperature. $\alpha$-bromoisobutyryl bromide was immobilized on cellulose membrane with hydroxyl groups directly from initiator immobilization step without prefunctionalizations. ${ }^{[11]}$ 
For benzyl chloride immobilization, the PET membranes after amination were soaked in 40 $\mathrm{mL} 0.125 \mathrm{~mol} / \mathrm{L}$ TEA MeCN solution at $0{ }^{\circ} \mathrm{C}$ for $30 \mathrm{~min} 100 \mathrm{~mL}$ in three neck flask under protection of argon, then $10 \mathrm{~mL} \mathrm{MeCN}$ containing $0.5 \mathrm{~mol} / \mathrm{L}$ 4-chloromethylbenzyol chloride was dropped into the $0{ }^{\circ} \mathrm{C}$ TEA solution slowly under gently stirring. After addition of 4chloromethylbenzyol chloride, the cooling bath was removed and the reaction lasted overnight. After reaction, the samples were washed twice in $\mathrm{MeCN}$, twice in ethanol and dried at $50^{\circ} \mathrm{C}$ overnight.

\subsubsection{Dithiocarbamate immobilization on PET membrane}

The DTC group for RAFT was immobilized on benzyl chloride modified membrane via membrane immersion in $0.15 \mathrm{~mol} / \mathrm{L}$ sodium diethyldithiocarbamat and 0.015 tert butyl ammonium bromide dried MeCN solution. After reaction, membranes were washed twice in $\mathrm{MeCN}$, twice in ethanol and dried at $50{ }^{\circ} \mathrm{C}$ under vacuument condition for $1 \mathrm{~h}$.

\subsubsection{Surface-initiated ATRP of tert.-butly methacrylate on PET membrane}

The ATRP set up and operations were described in details by Friebe. ${ }^{[11,65]}$ ATRP was carried out using a $1.5 \mathrm{~mol} / \mathrm{L}$ solution of $t \mathrm{BMA}$ in an $\mathrm{MeCN} /$ water mixture $(\mathrm{V}: \mathrm{V}=9: 1)$ with a catalyst system consisting of $\mathrm{CuCl} / \mathrm{CuCl}_{2} / \mathrm{Bpy}$. The molar ratio in all experiments was [tBMA]:[CuCl]:[CuCl$]:[\mathrm{Bpy}]=75: 1: 0.1: 2.75$. Other conditions for ATRP optimization are shown in Table 3.1. A quenching solution was used to stop the polymerization and ensure that the chain ends were terminated with $\mathrm{C}-\mathrm{Br}$; the membranes were immersed in $20 \mathrm{~mL}$ methanol solution containing $100 \mathrm{mg}$ copper(II) bromide and $250 \mu \mathrm{L}$ PMDETA for $1 \mathrm{~h}$. Thereafter, the membranes were washed in methanol for $1.5 \mathrm{~h}$ and then dried in a vacuum oven. The degree of grafting (DG) was calculated according to:

$$
D G=\frac{\mathrm{m}_{\mathrm{gr}}-\mathrm{m}_{0}}{A_{\text {spec }}}
$$

Where $m_{0}$ is weight of the sample before ATRP and $m_{g r}$ is the weight after ATRP (blance Genius with accuracy $0.01 \mathrm{mg}$, Satorius, Germany), $A_{\text {spec }}$ is the specific surface area of the base membrane (cf. section 3.3.2). 
Table 3.1 ATRP conditions used in this work

\begin{tabular}{c|c|c|c}
\hline$t$ BMA [mol/L] & Reaction system & Mol ratio & solvent \\
\hline 2 & $t \mathrm{BMA} / \mathrm{CuCl} / \mathrm{Me}_{6}$ TREN & $100: 1: 1$ & Acetone+water \\
\hline 2 & $t \mathrm{BMA} / \mathrm{CuCl} / \mathrm{PMDETA}$ & $100: 1: 1$ & Acetone+water \\
\hline 2 & $t \mathrm{BMA} / \mathrm{CuCl} / \mathrm{Bpy}$ & $100: 1: 2 / 2.5 / 2$ & Acetone+water \\
\hline 1.5 & $t \mathrm{BMA} / \mathrm{CuCl} / \mathrm{Bpy}$ & $75: 1: 2$ & Acetone+water \\
\hline 1 & $t \mathrm{BMA} / \mathrm{CuCl} / \mathrm{Bpy}$ & $50: 1: 2$ & Acetone+water \\
\hline 1.5 & $t \mathrm{BMA} / \mathrm{CuCl} / \mathrm{CuCl}_{2} / \mathrm{Bpy}$ & $75: 1: 2.5$ & MeCN+water \\
\hline 1.5 & $t \mathrm{BMA} / \mathrm{CuCl} / \mathrm{CuCl}_{2} / \mathrm{Bpy}$ & $75: 1: 0.1: 2.75$ & MeCNe+water \\
\hline 1.5 & $t \mathrm{BMA} / \mathrm{CuCl} / \mathrm{PMDETA}$ & $75: 1: 1.25$ & MeCN \\
\hline
\end{tabular}

\subsubsection{Hydrolysis of grafted poly(tert.-butly methacrylate) to poly(methacrylic acid)}

To obtain the scaffold PET-g-PMAA membranes, the PET-g-PtBMA membranes were soaked in $4 \mathrm{~mL}$ of a $50 \%$ solution of trifluoroacetic acid in $\mathrm{MeCN} /$ water $(\mathrm{V}: \mathrm{V}=1: 1)$ for $18 \mathrm{~h}$ at $50^{\circ} \mathrm{C}$ with shaking rate of $150 \mathrm{rmp}$. For the cellulose- $g$-PtBMA membrane's hydrolysis, 35\% trifluoroacetic acid was used and the reaction time was reduced to $14 \mathrm{~h}$. After hydrolysis the samples were immersed in water twice to remove the residues of trifluoroacetic acid. Then they were immersed for $30 \mathrm{~min}$ in ethanol, and subsequently the samples were dried for $1 \mathrm{~h}$ at $40^{\circ} \mathrm{C}$ in vacuum oven.

\subsubsection{Preparation of lysozyme/immunoglobin G imprinted membraned via UV- initiated grafting/-crosslinking copolymerization}

The template adsorption on the PET-g-PMAA or Cellulose-g-PMAA membranes was carried out in $5 \mathrm{~mL}$ of a $1 \mathrm{~g} / \mathrm{L}$ solution of Lyz or IgG in water with $\mathrm{pH} \approx 7.0$ for $20 \mathrm{~h}$. Thereafter, the membranes were washed in water $(\mathrm{pH} \approx 7.0)$ for $1 \mathrm{~h}$ (twice) to remove protein which was not bound to the membrane. Subsequently, the samples were wetted with the grafting/crosslinking solution, i.e. a $0.3 \mathrm{~mol} / \mathrm{L}$ solution of the monomers of AAm and MBAA (in varied ratios). At first the membranes were mounted into a filter holder (Swinnex, Millipore, Ireland) and the solution was gently pushed through the membrane with a $20 \mathrm{~mL}$ syringe (Omnifix, B.Braun, Germany) for 3 times, then the membranes were immersed in the solution for $1.5 \mathrm{~h}$. The UV-initiation with UVA light of an intensity of about $24 \mathrm{~mW} / \mathrm{cm}^{2}$ was done in a UV irradiation system UVA Cube 2000 (Hönle AG, Germany) for imprinting of Lyz and IgG on PET membrane, UVA Pint 100-200 (Hönle AG, Germany) was used for imprinting 
of Lyz on cellulose membrane with UV-intensity (UVA) about $71 \mathrm{~mW} / \mathrm{cm}^{2}$. Afterwards, the grafted membranes were taken out and washed 3 times in water to remove the unreacted monomers. Finally, the template Lyz was eluted from the membranes using $5 \mathrm{mmol} / \mathrm{L}$ phosphate buffer containing $1 \mathrm{~mol} / \mathrm{L} \mathrm{NaCl}(\mathrm{pH}=7.0)$ via shaking with a rate of $250 \mathrm{rpm}$ for 20 h. Non imprinted polymer (NIP) membranes were prepared in the absence of template by using otherwise the same procedure and conditions as described above. All membranes were stored in $5 \mathrm{mmol} / \mathrm{L}$ phosphate buffer, $\mathrm{pH}=7.0$, until further use.

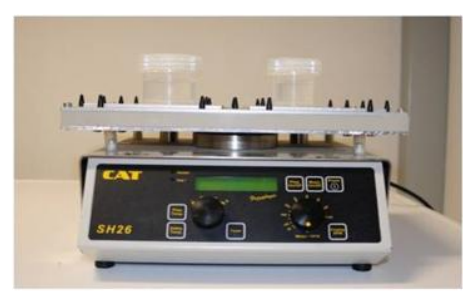

Protein adsorption on scaffold $200 \mathrm{rpm}, 20^{\circ} \mathrm{C}$

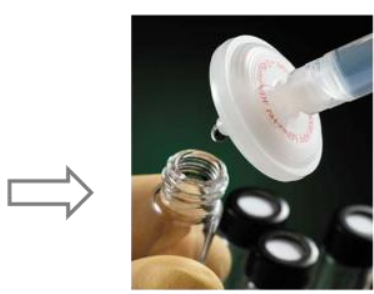

Membrane wetting with UV-polymerization solution

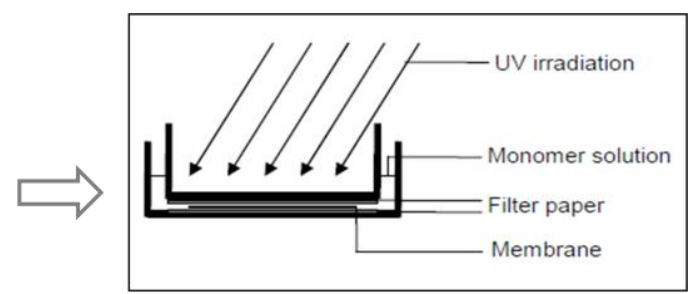

UV-grafting/crosslinking

Figure 3.1 Schematic illustration of protein imprinting process.

\subsubsection{Surface grafting via photo-RAFT}

Before UV-initiation, DTC immobilized PET membrane ( $\varnothing 25 \mathrm{~mm}$ ) was immersed in $4 \mathrm{~mL}$ AAm MeCN solution in a Petri dish $(\varnothing 60 \mathrm{~mm})$ and fixed between two sheets of filter paper for $30 \mathrm{~min}$ (cf. Figure 3.1). The Photo-RAFT grafting was performed in UVA Cube 2000 with UVA light of an intensity of about $24 \mathrm{~mW} / \mathrm{cm}^{2}$. After reaction, the membrane samples were washed twice in $\mathrm{MeCN}$ and twice in ethanol and finally fried in oven at $50{ }^{\circ} \mathrm{C}$. The degree of grafting (DG) was calculated as described in 3.2.3. For reaction in AAm aqueous solution, the DTC immobilized membranes were presoaked in $4 \mathrm{~mL}$ acetone for $15 \mathrm{~min}$, then washed in $\mathrm{mL} \mathrm{AAm}$ aqueous solution twice $(5 \mathrm{~min}$ ) before UV-initiation.

\subsection{Characterizations}

\subsubsection{Pore size distribution}

Pore size distribution was analyzed by the PMI capillary flow porometer (Porous Materials, Inc., Ithaca, NY). For membrane samples with a diameter of $25 \mathrm{~mm}$, gas flow as a function of 
the trans-membrane pressure was measured, first through the dry membrane, then after wetting the membrane with 1,1,2,3,3,3-hexafluoropropene ("Galwick", PMI; surface tension $16 \mathrm{dyn} / \mathrm{cm})$. The pore size distribution and the average pore diameter were calculated by the PMI software. The grafted polymer layer thickness in dry state, $L_{d r y}$, was calculated according to:

$$
\mathbf{L}_{\mathrm{dry}}=\mathbf{r}_{\mathbf{p}}-\mathbf{r}_{\text {modified }}
$$

where $r_{p}$ is the average pore radius of the base membrane, and $\mathbf{r}_{\text {modified }}$ is the average pore radius of the modified membrane.

\subsubsection{Liquid permeability measurement and subsequent analyses}

Flux measurement for the base membrane was performed with $0.1 \mathrm{~mol} / \mathrm{L} \mathrm{NaCl}$ in water solution at $25^{\circ} \mathrm{C}$ by using a batch stirred cell with $10 \mathrm{~mL}$ volume and $3.46 \mathrm{~cm}^{2}$ effective membrane area (Amicon Model 8010, Millipore). To determine the pore density, $\rho_{p}$, of the TE PET membrane, an equation derived from Hagen-Poiseuille law in a form suited for analysis of isoporous membranes was used:

$$
\boldsymbol{\rho}_{\mathrm{p}}=\frac{128 \cdot \mathbf{V} \cdot \boldsymbol{\eta} \cdot \mathbf{L}}{\mathbf{A} \cdot \mathbf{\pi} \cdot \Delta \mathbf{t} \cdot \Delta \mathbf{p} \cdot d^{4}}
$$

where $\mathbf{V}$ is the permeate volume, $\eta$ is the viscosity of the permeate, $\mathrm{L}$ is capillary length (thickness of the membrane), $\mathrm{A}$ is the membrane area, $\Delta \mathrm{t}$ is the time interval, $\Delta \mathrm{p}$ is the transmembrane pressure, and $\mathbf{d}$ is the pore diameter. The mean pore diameter of the base membrane had been obtained from gas flow / liquid dewetting permporometry (cf. section 2.3.1). With help of the pore density and the mean pore diameter, the specific surface area of the TE membrane was calculated, assuming cylindrical geometry of the membrane pores and equal sizes of all pores:

$$
A_{\text {spec }}=2 \mathbf{A}+\pi \mathbf{d}_{\mathbf{m}} \cdot \mathbf{L}+\left(\boldsymbol{\rho}_{\boldsymbol{p}} \cdot \boldsymbol{A}\right) \cdot\left[\pi \cdot \mathbf{d} \cdot \mathbf{L}-2 \pi \cdot\left(\frac{\mathbf{d}}{2}\right)^{2}\right]
$$

where $d_{m}$ is the diameter of the membrane sample.

The permeability of the modified membranes were measured by using a mixture of ethanol and water $(\mathrm{V}: \mathrm{V}=1: 1)$ at $\mathrm{pH}=7.2$ to ensure pore wetting, irrespective the hydrophilicity or 
hydrophobicity of the different membranes. Effective (hydrodynamic) pore diameter/radius of the modified membranes could then be deduced under the assumption that the pore density $\rho_{p}$ remained unchanged. The hydrodynamic thickness of the grafted polymer layer on the pore walls, $L_{\text {hydro, }}$ was calculated as below:

$$
\mathbf{L}_{\text {hydro }}=\mathbf{r}_{\mathbf{p}}-\frac{1}{2} \cdot\left(\frac{128 \cdot \mathbf{V} \cdot \mathbf{\eta} \cdot \mathbf{L}}{\mathbf{A} \cdot \pi \cdot \Delta \mathbf{t} \cdot \Delta \mathbf{p} \cdot \mathbf{\rho}_{\mathbf{p}}}\right)^{\frac{1}{4}}
$$

where $\mathbf{r}_{\mathbf{p}}$ is the pore radius of the unmodified membrane.

\subsubsection{Contact angle measurements}

Contact angles were measured via the static sessile drop method using an optical contact angle measurement system (OCA 15 plus, Dataphysics, Germany). A drop of water $(5 \mu \mathrm{L})$ was injected using a syringe onto the sample surface. A fitting method based on the YoungLaplace model was used to calculate the contact angle from the images obtained with the goniometer. Data for 5 drops on different locations were averaged to obtain the contact angle of one membrane sample.

\subsubsection{Specific surface area of membranes measurement via BET}

After washing with methanol and ethanol and overnight drying at $50{ }^{\circ} \mathrm{C}$, base TE PET and cellulose membranes (about $100 \mathrm{mg}$ for each measurement) were analyzed via SA 3100 (Beckmann-Coulter $\mathrm{GmbH}$, Krefeld, Germany). The specific surface area was characterized via nitrogen adsorption and calculated according to the isotherm of Brunauer, Emmett and Teller (BET) model.

\subsubsection{Carboxyl group density measurement}

The carboxyl group density on the membrane surface was characterized by reversible ionic dye binding using thionine acetate. ${ }^{16}$ Membrane samples were shaken in a solution of 10 $\mathrm{mg} / \mathrm{L}$ thionine acetate in ethanol for $20 \mathrm{~h}$ at room temperature, rinsed three times with ethanol for $30 \mathrm{~s}$ and then immersed in $10 \mathrm{~mL}$ of a solution of $0.01 \mathrm{~N} \mathrm{HCl}$ in a $1: 1$ mixture of ethanol and water. After shaking for $2 \mathrm{~h}$, the concentration of the released thionine actetate 
was determined spectrophotometrically at $594 \mathrm{~nm}$ (Cary 50 Probe UV/Vis spectrophotometer, Varian Deutschland GmbH, Darmstadt, Germany).

\subsubsection{ATR/FTIR}

The IR spectra of modified membranes were obtained by the Jasco FT/IR-430 (Japan) Fourier Transform Infrared spectroscopy equipped with MIRacle ${ }^{T M}$ ATR unit. Software Spetra Mange was used to record and analyze the data.

\subsubsection{X-ray photoelectron spectroscopy}

A Physical Electron 5800 ultrahigh vacuum XPS-Auger spectrometer was used for the measurement at a $45^{\circ}$ takeoff angle. Twenty high-resolution scans focused on the nitrogen (395 - $405 \mathrm{eV})$, the bromine $(65-77 \mathrm{eV})$, and sulfur (155 - $175 \mathrm{eV})$ regions were performed to observe changes after the functionalization steps. Charge compensation was achieved by using double beam neutralizer.

\subsubsection{Static binding capacity of proteins}

Solutions were prepared by dissolving the proteins in phosphate buffer solution $(5 \mathrm{mmol} / \mathrm{L}$, $\mathrm{pH}=7.0$ ). The protein binding was carried out by soaking the membrane sample in protein solution of $0.2 \mathrm{~g} / \mathrm{L}$ at $20^{\circ} \mathrm{C}$ with shaking rate $250 \mathrm{rpm}$. For PET membrane $5 \mathrm{~mL}$ solution was used and for cellulose membrane $10 \mathrm{~mL}$ was used. After $1 \mathrm{~h}$, the membrane samples were taken out of the solution and washed in $20 \mathrm{~mL}$ phosphate buffer ( $1 \mathrm{~h}$, twice). Thereafter, the membranes were soaked in $5 \mathrm{~mL}$ phosphate buffer containing $1 \mathrm{~mol} / \mathrm{L} \mathrm{NaCl}$ for $1 \mathrm{~h}$ to elute the bound protein at $20{ }^{\circ} \mathrm{C}$ with shaking rate $250 \mathrm{rpm}$. The Lyz, IgG, and HSA concentration in the single protein solutions after binding and elution were determined photometrically using the $\mu$ Quant microplate reader (Bio-Tek Instruments, Inc.) after staining by using the BCA or microBCA assay (Thermo Scientific). The CyC concentration was directly measured by UV-Vis spectroscopy at $410 \mathrm{~nm}$. The concentration of Lyz in a mixture with CyC was determined through Lyz enzyme activity, which is based on following the lysis of Micrococcus lysodeikticus cell walls. Micrococcus lysodeiktikus cell wall material was suspended in $5 \mathrm{mM}$ phosphate buffer $\mathrm{pH} 7.0$ in a concentration of $1 \mathrm{mg} / \mathrm{mL}$. Activity was determined by addition 
of $1.5 \mathrm{ml}$ of sample to $1.5 \mathrm{~mL}$ of suspension in a cuvette at room temperature. Changes in turbidity were measured after 2 minutes incubation time by UV/Vis-spectrophotometer at $450 \mathrm{~nm}$. Lys concentrations were obtained based on a calibration curve.

Protein binding capacity (C) was calculated according to:

$$
C=\frac{\mathrm{m}_{\mathrm{p}}}{\mathrm{V}_{\mathrm{m}}}
$$

where $m_{p}$ is the weight of bound protein and $V_{m}$ is the membrane volume.

The selectivity of Lyz to CyC (S) was defined as:

$$
S=\frac{\mathrm{C}_{\mathrm{Lyz}}}{\mathrm{C}_{\mathrm{CyC}}}
$$

To obtain binding isotherms, MIP as well as its corresponding NIP membranes were immersed in Lyz solutions of various concentrations ( 0.01 to $0.2 \mathrm{~g} / \mathrm{L}$ ) in phosphate buffer $(\mathrm{pH}$ $=7.0$ ) for $1 \mathrm{~h}$ at $20^{\circ} \mathrm{C}$. After washing with buffer and elution with buffer containing $1 \mathrm{~mol} / \mathrm{L}$ $\mathrm{NaCl}$, the amount of adsorbed Lyz was measured. 


\section{Chapter 4 Results and discussion}

\subsection{Characterizations of base PET membrane}

It is important to know the information about the base membrane as much as possible before application in laboratory. The information from manufacturer usually includes some technical inaccuracy; therefore, it is necessary to characterize the property of the commercial membrane before modification and its later evaluation. The TE PET 400 membrane was received from the manufacturer with the information: pore diameter 400 $\mathrm{nm}$, porosity about $13 \%$, pore density 100 million pores $/ \mathrm{cm}^{2}$, and thickness $23 \mu \mathrm{m}$.

At first the pore size distribution of the base TE PET400 membrane was measured by gas flow / pore dewetting permporometry (Figure 4.1).

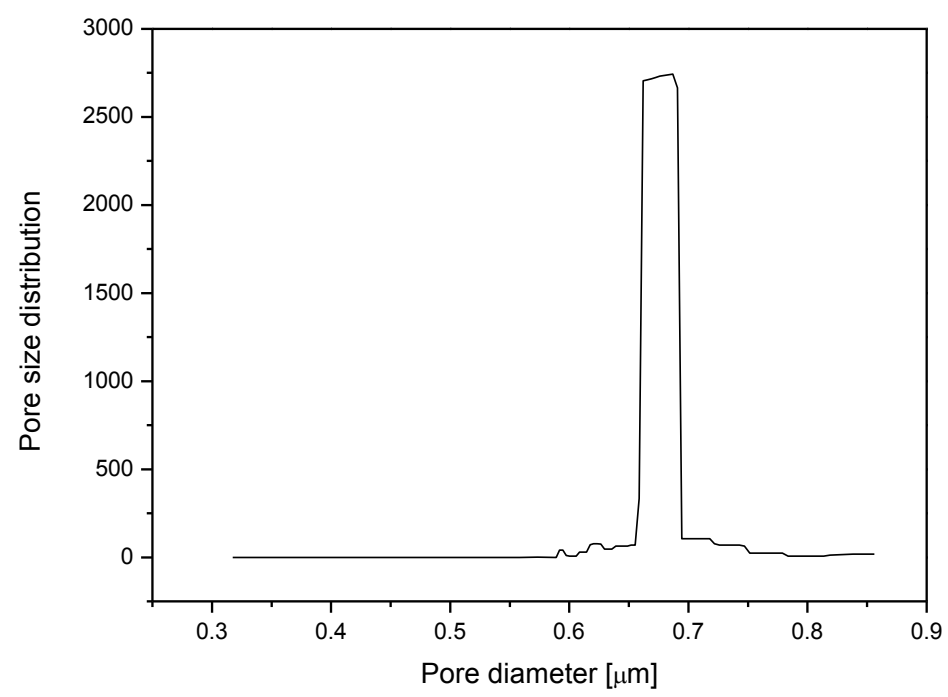

Figure 4.1 Pore size distribution measured by liquid dewetting permporometry for TE PET400 basis membrane.

The distribution of the pore size was narrow with an average diameter about $675 \mathrm{~nm}$ which was larger than the nominal diameter $400 \mathrm{~nm}$ from the manufacturer. In comparison with the old product which has been used by Ulbricht and his coworkers, ${ }^{[10-11]}$ the distribution of pore size has been extended but is still acceptable. The membrane morphology was further characterized by SEM. The isotropic structure of the TE PET400 membrane was observed as it was claimed by Oxyphen $\mathrm{GmbH}$. This is a key precondition to use Hagen-Poiseuille model 
in the further permeability experiment and calculation. The partial overlap of the pore structure leads to an extension of the pore size distribution.
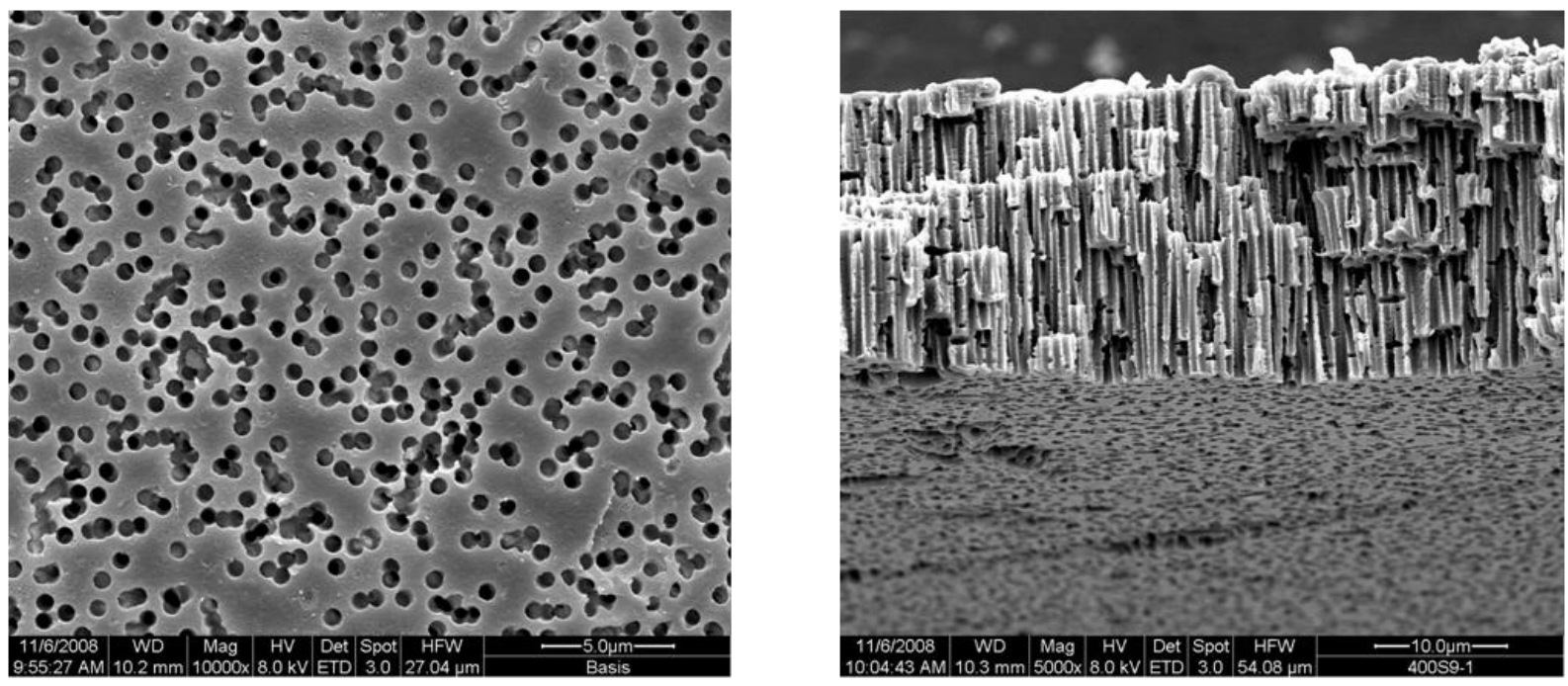

Figure 4.2 SEM image of TE PET400 base membrane: top view (left) and cross-section (right).

The permeability of base membrane was measured with Milli_Q water containing $0.1 \mathrm{~mol} / \mathrm{L}$ $\mathrm{NaCl}$. The pore density is $78.7^{*} 10^{6}$ pore $/ \mathrm{cm}^{2}$ calculated by the equation 3 which is smaller than the manufacturing value $100 * 10^{6}$ pore $/ \mathrm{cm}^{2}$. Porosity is about $28 \%$ which is larger than the manufacturing value $12.6 \%$. The specific surface area was calculated by the equation 4 , and the value is $194.34 \mathrm{~cm}^{2}$ for the membrane sample with diameter $25 \mathrm{~mm}$.

Table 4.1 Comparison of the data from manufacturer and measurement in laboratory.

\begin{tabular}{c|c|c|c|c|c}
\hline TE PET 400 & Pore type & $\begin{array}{c}\text { Pore diameter } \\
{[\mathrm{nm}]}\end{array}$ & $\begin{array}{c}\text { Pore density } \\
* 10^{6} / \mathrm{cm}^{2}\end{array}$ & $\begin{array}{c}\text { Porosity } \\
{[\%]}\end{array}$ & $\begin{array}{c}\text { Specific surface } \\
(\varnothing 25 \mathrm{~mm}) \\
{\left[\mathrm{cm}^{2}\right]}\end{array}$ \\
\hline Manufacturer & Cylindrical & 400 & 100 & 12.6 & ----- \\
\hline Measured in Lab. & Cylindrical & 675 & 78.7 & 28 & 194.34 \\
\hline
\end{tabular}




\subsection{ATRP initiator immobilization on PET membrane}

The surface initiation of ATRP initiator group is much different from the bulk polymerization. The initiation efficiency of surface grafting is very low and only about $10 \%{ }^{[133]}$ In order to obtain the grafted chains with high density (brush regime), the initiator bromide was immobilized with high density on the membrane surface via 4 steps: oxidation, activation, amination, and ATRP initiator immobilization, as shown in Figure 4.3.

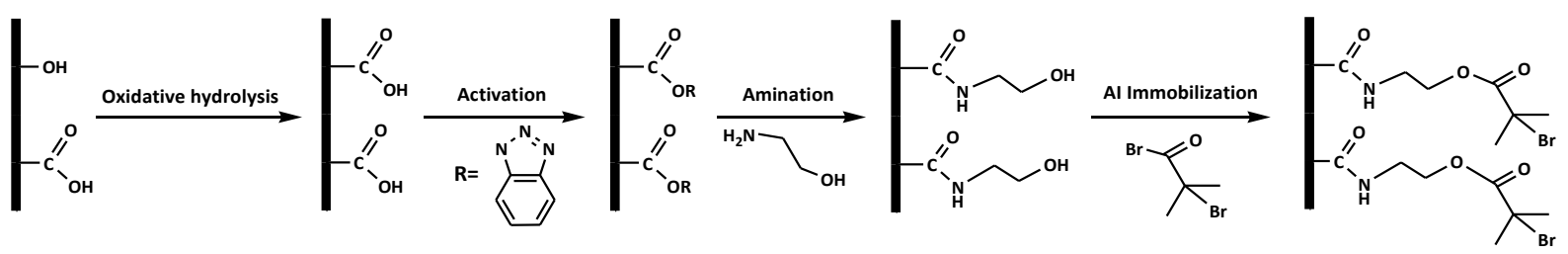

Figure 4.3 Immobilization of ATRP initiator on TE PET membrane surface.

At first, the density of the hydroxyl group was improved via oxidative hydrolysis, activation, and amination. The conversion of the carboxyl group was characterized via the reversible adsorption of the cationic dye thionin acetate (Figure 4.4).

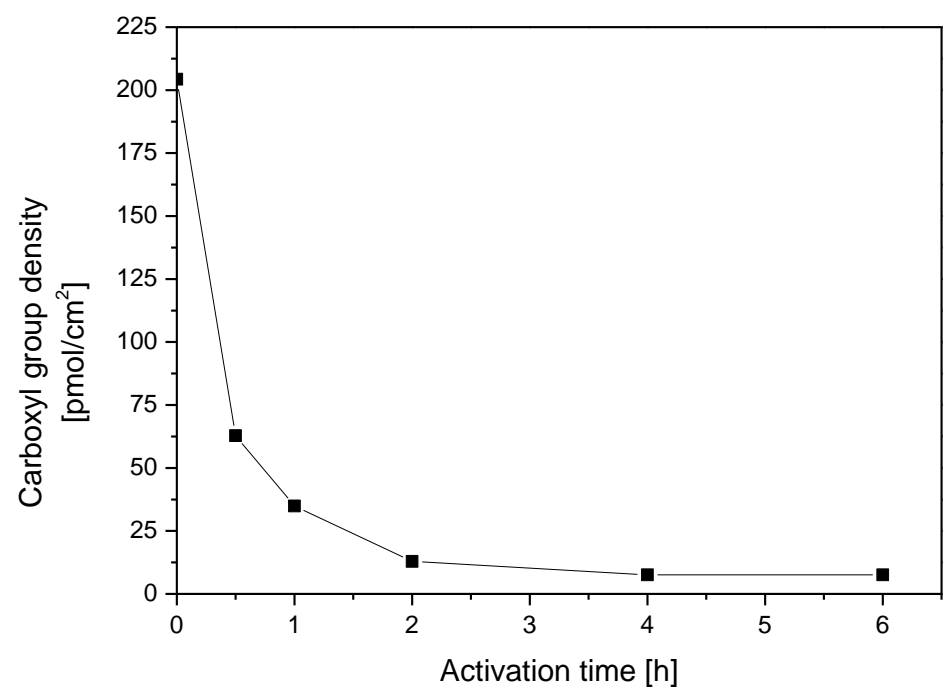

Figure 4.4 Carboxyl group densities on the PET membrane surface after reaction with ethanolamine ("amination") with different activation times.

The density of the surface carboxyl group was almost doubled by the oxidative hydrolysis, from $107 \mathrm{pmol} / \mathrm{cm}^{2}$ of the base membrane to $204 \mathrm{pmol} / \mathrm{cm}^{2}$. The improved density of carboxyl group was a support to improve the hydroxyl group density through the subsequent activation and amination. About $70 \%$ of the carboxylic acid groups were converted after 0.5 
hour activation. After 6 hour activation and subsequent amination, the constant conversion of the carboxyl acid groups of about $97 \%$ showed that the activation had apparently reached the saturated level. The conversion of carboxyl groups had improved about 1.4 times through extending the activation time from $0.5 \mathrm{~h}$ to $6 \mathrm{~h}$.

The improvement of the carboxyl group conversion was further testified via XPS measurements of the nitrogen after activation and amination (Figure 4.5).

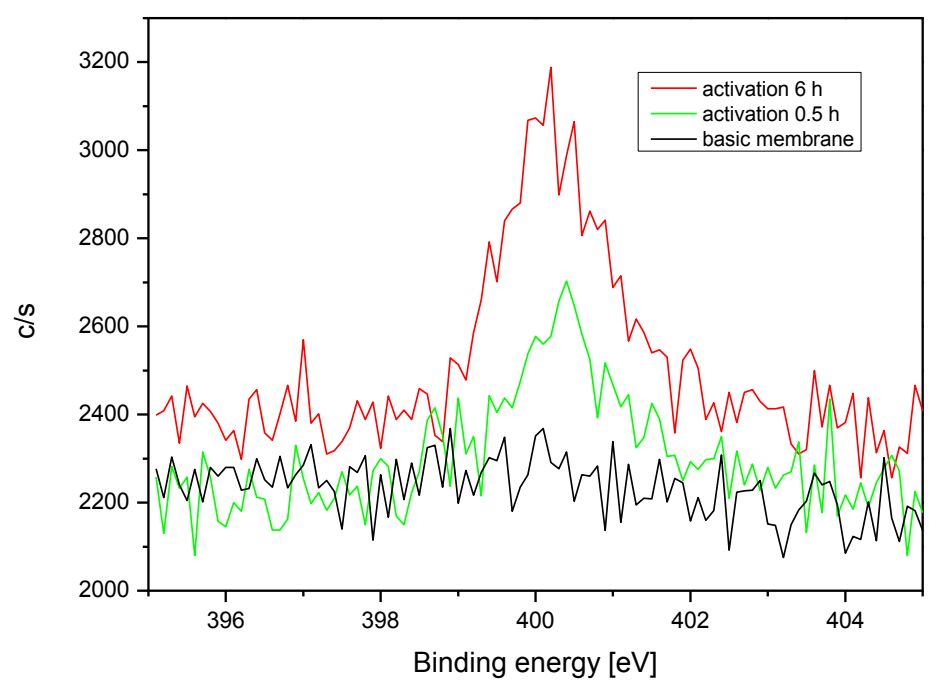

Figure 4.5 XPS spectra of the nitrogen region of different membranes: black ... base TE PET membrane; green ... TE PET after amination with $0.5 \mathrm{~h}$ activation time; red ... TE PET after amination with $6 \mathrm{~h}$ activation time.

Base TE PET does not contain nitrogen; the same had been observed for materials after oxidative hydrolysis. The result of XPS revealed the same tendency compared to carboxylic acid group conversion via reversible dye adsorption. It was feasible to improve the density of hydroxyl groups on the PET membrane surface via extending activation time; based on nitrogen content on the membrane surface it was increased about 2.5 times through extending activation time from $0.5 \mathrm{~h}$ to $6 \mathrm{~h}$, which is in qualitative agreement with the result of carboxyl group conversion tendency (1.4 times) (cf. Figure 4.5). The difference is related to the different measuring methods, indirect analysis via dye binding vs. direct spectroscopic analysis. The density of hydroxyl group via amination is equal to the nitrogen content, it is proved that this strategy to improve the hydroxyl group is feasible. This result was also 
further supported by the data on subsequent ATRP initiator immobilization; analysis had been done via XPS spectra of the bromine region (Figure 4.6).

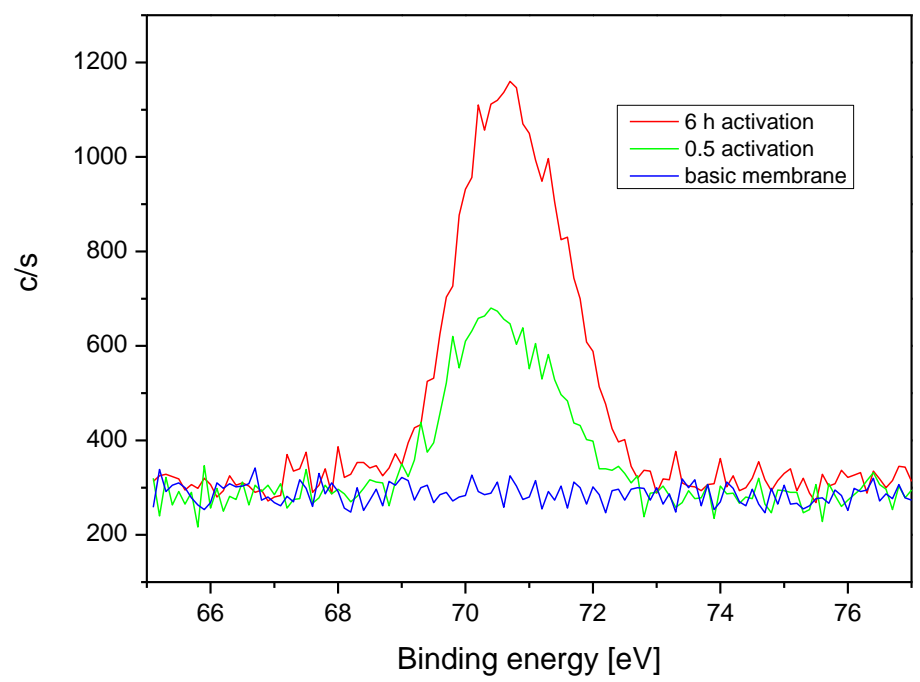

Figure 4.6 XPS spectra of the bromine region of different membranes: blue ... base TE PET membrane; green ... TE PET after $\alpha$-bromoisobutyryl group immobilization with previously $0.5 \mathrm{~h}$ activation time; red ... TE PET after $\alpha$-bromoisobutyryl group immobilization with previously $6 \mathrm{~h}$ activation time.

The density of $\alpha$-bromoisobutyryl ATRP initiator was improved about 2.6 times through extending the activation time from $0.5 \mathrm{~h}$ to $6 \mathrm{~h}$, which was in quantitative agreement with the conclusions from XPS result for nitrogen (cf. Figure 4.5). $6 \mathrm{~h}$ activation was chosen for the further Al immobilization. The improved bromide density could lead to the surface grafting with high density polymer chains and provides also a potential to optimize the density of grafted polymer chains to meet some special requirements via the diluent method, i.e. using a mixture of not reactive acryl bromide and $\alpha$-bromoisobutyryl bromide during the ATRP initiator immobilization to adjust the density of active initiator ${ }^{[14,133]}$.

For the later work, a high density of grafted polymer chains in brush regime is required, because it prefers to adsorb the protein via multi chain attributes. This leads to form a more stable complex and should be beneficial to produce a protein imprinted structure with high specific recognition property ("3D model"). Protein binding in 3D grafted polymer layers has been discussed by Kato et al. ${ }^{[134]}$ If the density of polymer chain is less dense, the polymer chains collapse on surface, the adsorption of protein by polymer chains is favored to form 
surface interaction ("2D model"). ${ }^{[12]}$ This is considered to be an obstacle for effective protein imprinting, because only part of the protein surface has been imprinted. 


\subsection{Surface initiated ATRP on PET membrane}

Following previous working experience from Friebe et al., ${ }^{[65]}$ the ATRP of tBMA was first tested in DMF using $2 \mathrm{M}$ tBMA with catalyst system consisting of $\mathrm{CuCl} / \mathrm{Me}_{6}$ TREN and molar ratio $[\mathrm{tBMA}]:[\mathrm{CuCl}]:\left[\mathrm{Me}_{6}\right.$ TREN] = 100:1:1 (Figure 4.7).

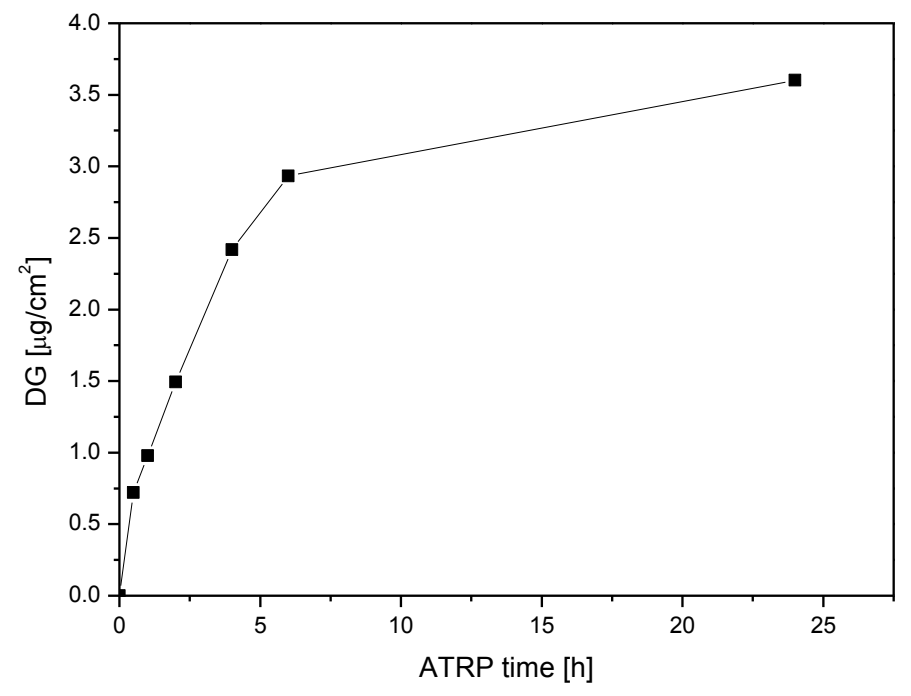

Figure 4.7 Degrees of grafting for PtBMA on PET membrane surface versus the reaction time of ATRP in $2 \mathrm{~mol} / \mathrm{L} t \mathrm{BMA}$ solution in DMF with a catalyst system of [tBMA]:[CuCl]:[ $\mathrm{Me}_{6}$ TREN] $=100: 1: 1$.

The solubility of $\mathrm{CuCl}$ in the reaction solution is poor, and a long time is needed to form the catalyst complex, some times more 30 min via observation of the dissolution of $\mathrm{Cu}$ particles in solution. Due to the degassing with argon, part of DMF evaporated and the reproducibility of the reaction was bad.

To improve the solubility of $\mathrm{CuCl}$ in the reaction solution, a mixture of acetone and water was used as solvent. ${ }^{[135]}$ The reactivities of different ligands, Bpy, PMDETA, and $\mathrm{Me}_{6}$ TREN, were tested in a $2 \mathrm{M} t \mathrm{BMA}$ reaction solution with catalyst system consisting of $\mathrm{CuCl} /$ ligand system and the molar ratio [tBMA]:[CuCl]:[Me 6 TREN/PMDETA] = 100:1:1 and [tBMA]:[CuCl]:[Bpy] = 100:1:2, respectively (Figure 4.8). 


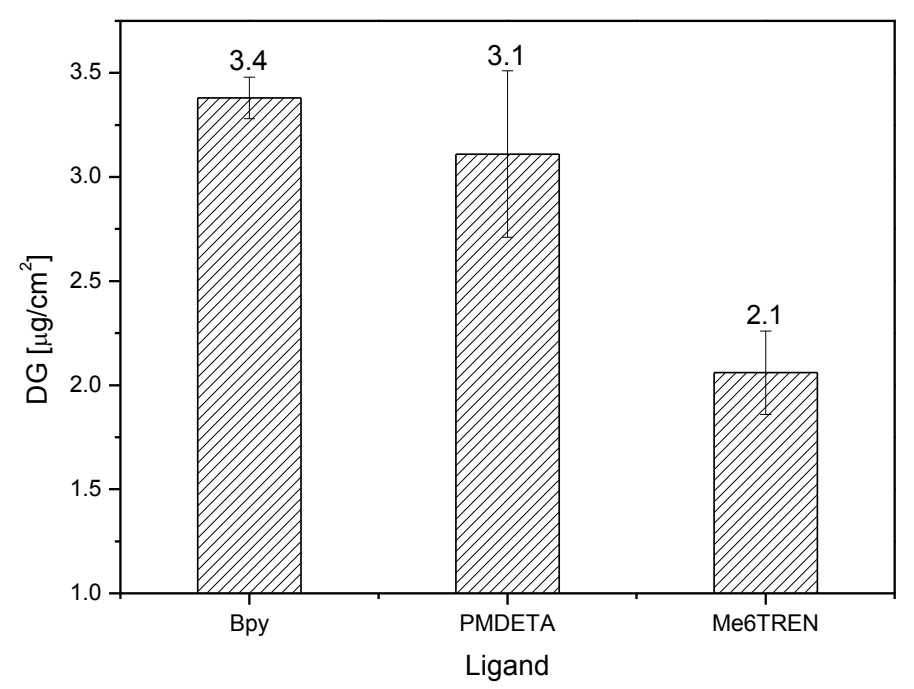

Figure 4.8 Degrees of grafting for PtBMA on PET membrane surface versus the different ligands of ATRP in $2 \mathrm{~mol} / \mathrm{L} t \mathrm{BMA}$ solution in acetone / water solution mixture ( $\mathrm{V}: \mathrm{V}=9: 1)$ with a catalyst system of $[t \mathrm{BMA}]:[\mathrm{CuCl}]:[\mathrm{Bpy}]=100: 1: 2$ and $[\mathrm{tBMA}]:[\mathrm{CuCl}]:\left[\mathrm{Me}_{6}\right.$ TREN/PMDETA] $=100: 1: 1$ at ambient temperature for $6 \mathrm{~h}$.

The reactivity sequence Bpy $>$ PMDETA $>$ Me6TREN is opposite to the activation rate constant (cf. Figure 2.9). The high activation constant led to a high propagating radical concentration during the ATRP, but the monomer addition of $t \mathrm{BMA}$ to the reactive sites was slow which results from the big steric effect and poor solubility of $t \mathrm{BMA}$ (slow diffusion rate). So the high concentration of propagating radical by system PMDETA and Me6TREN preferred to form bimolecular combination and led to a lower reaction rate than Bpy system. The solubility of Bpy in acetone is good, and the introduction of water with high polarity can enhance the complex efficiency of $\mathrm{Bpy} / \mathrm{Cu}(\mathrm{l})$ to form more reactive catalyst( Scheme 4.1). ${ }^{[136]}$ 


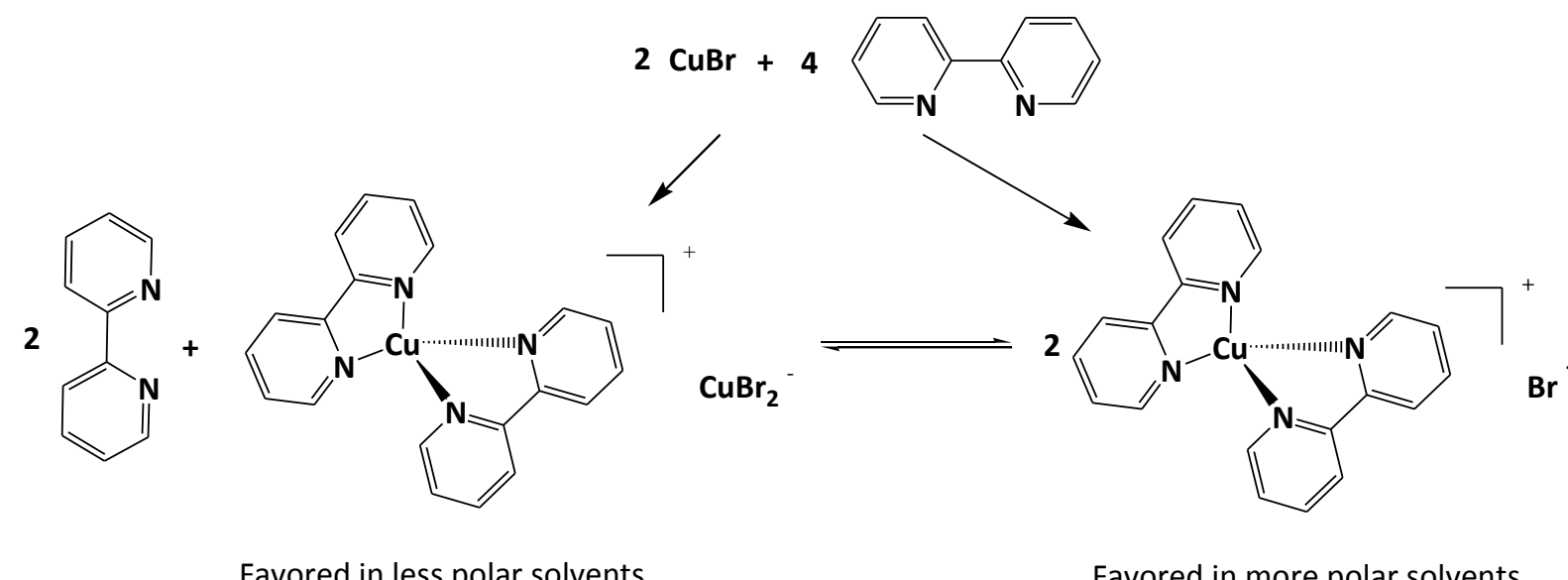

Scheme 4.1 Complex state of Bpy with $\mathrm{CuBr}$ in the solvents with different polarities.

Acetone is easy to evaporate from the mixture solution under the bubbling with argon, and the polarity of the aqueous solution increased, and meanwhile the solubility of the hydrophobic monomer tBMA was reduced. Sometimes part of the tBMA was separated out from the bulk solution during bubbling process, which leads to a poor control over ATRP. It is feasible to avoid the partial solution of $t \mathrm{BMA}$ by reducing the concentration of the $t \mathrm{BMA}$ in the reaction solution. The reactivity of the system with reduced concentration was tested and the results are shown as follows (Figure 4.9).

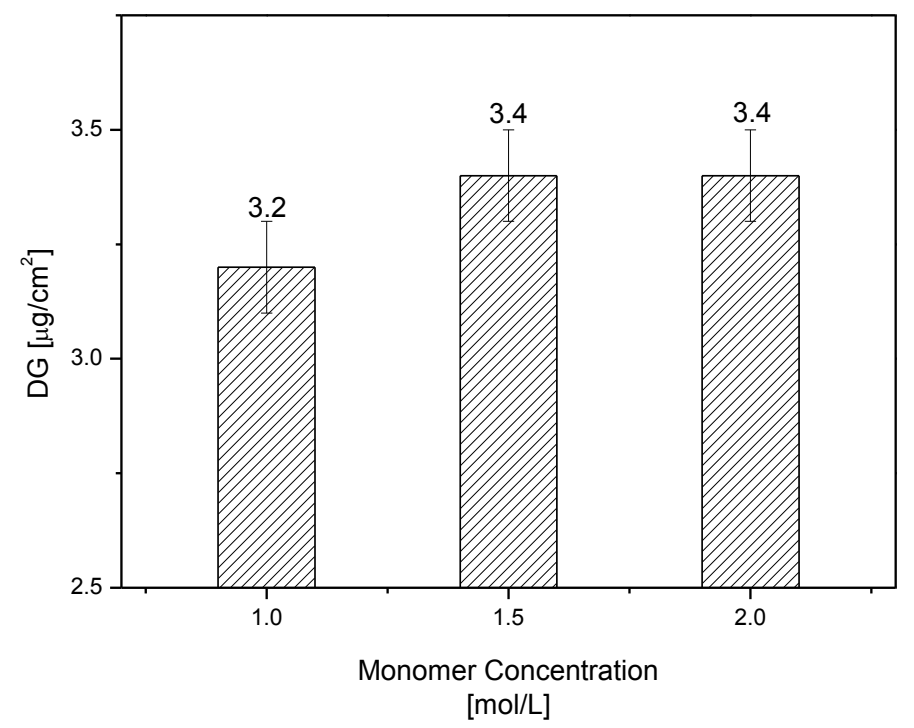

Figure 4.9 Degree of grafting for PtBMA on PET membrane surface versus the different $t \mathrm{BMA}$ concentrations in acetone / water solution mixture (V:V $=9: 1)$ with a catalyst system of $[$ tBMA]:[CuCl]:[Bpy] = 100/75/50:1:2 at ambient temperature for $6 \mathrm{~h}$. 
The effect of $t$ BMA concentration reduction from 2 to $1 \mathrm{~mol} / \mathrm{L}$ has been compensated by increase of polarity of the reaction solution (less polar tBMA) and showed almost no influence on the reactivity. The positive effect on catalyst complex has been discussed in section 2.3.4. ${ }^{[136]}$ The ATRP system prefers to take the ISET route rather than OSET route in more polar solution. The viscosity of $t \mathrm{BMA}$ is much higher than that of acetone and water. The reduction of $t B M A$ content led to a less dense reaction phase and results in a better diffusion for the $t B M A$ to the reactive sites. Too low concentration of $t B M A$ has shifted the reaction equilibrium, which dominated the change of polarity and resulted in a slower reaction rate. After the concentration optimization, $1.5 \mathrm{~mol} / \mathrm{L}$ of $t B M A$ was chosen for the further investigations.

Following the results from Nanda et al., ${ }^{[136]}$ increase of the ratio of Bpy to $\mathrm{Cu}(\mathrm{I})$ can improve the reactivity of ATRP. Similar work has been done on the surface grafting in this project. The relationship between ATRP reactivity and ratio of $\mathrm{Bpy}$ to $\mathrm{Cu}(\mathrm{I})$ was demonstrated in Figure 4.10.

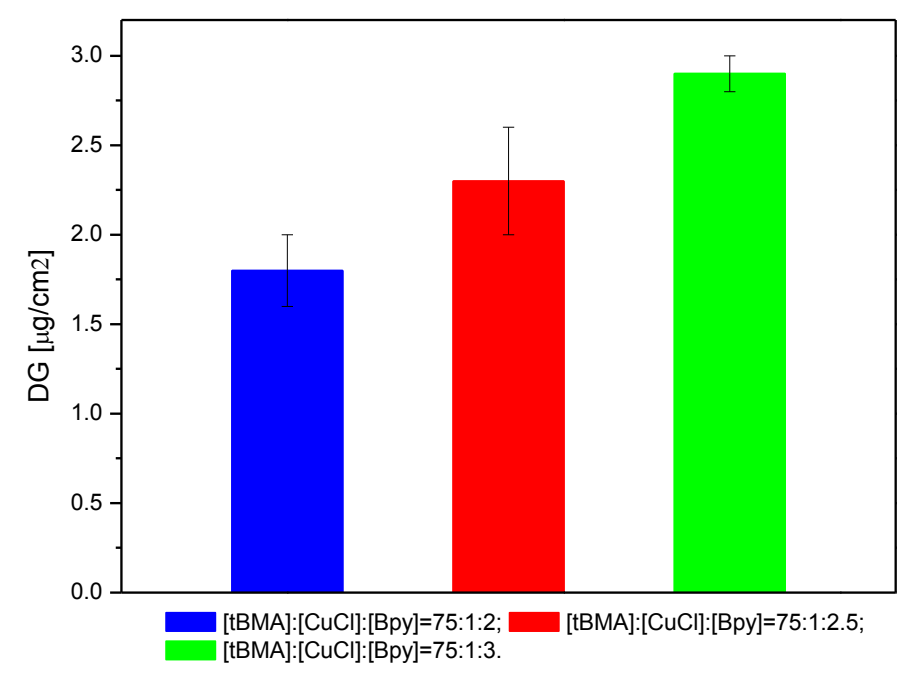

Figure 4.10 Degree of grafting for PtBMA on PET membrane surface versus the different ratios of $\mathrm{Bpy}$ to $\mathrm{Cu}(\mathrm{I})$ in $1.5 \mathrm{~mol} / \mathrm{L}$ tBMA acetone / water solution mixture $(\mathrm{V}: \mathrm{V}=9: 1)$ with a catalyst system of [tBMA]:[CuCl]:[Bpy] $=75: 1: 2 / 2.5 / 3$ at ambient temperature for $6 \mathrm{~h}$.

Increasing the ratio of $\mathrm{Cu}(\mathrm{I})$ to Bpy from 1:2 to 1:3 led to an increase of the ATRP reactivity, which is different to the result of Nanda et al. that the reactivity reached the saturation with the ratio $[\mathrm{CuCl}]:[\mathrm{Bpy}]=2$ by bulk ATRP of tert butyl acrylate in MeCN. ${ }^{[136]}$ The two phase 
reaction by SI-ATRP needs an additional step which is transport of reagent to reactive surface. High concentration of the catalyst complex benefited to a quicker transport driven by the concentration gradient, so the reactivity of the SI ATRP increased until the ratio of $[\mathrm{CuCl}]:[\mathrm{Bpy}]=3$.

MeCN aqueous solution which is considered to have a good wetting property to PET material was also used as solvent for SI-ATRP of tBMA (Figure 4.11).

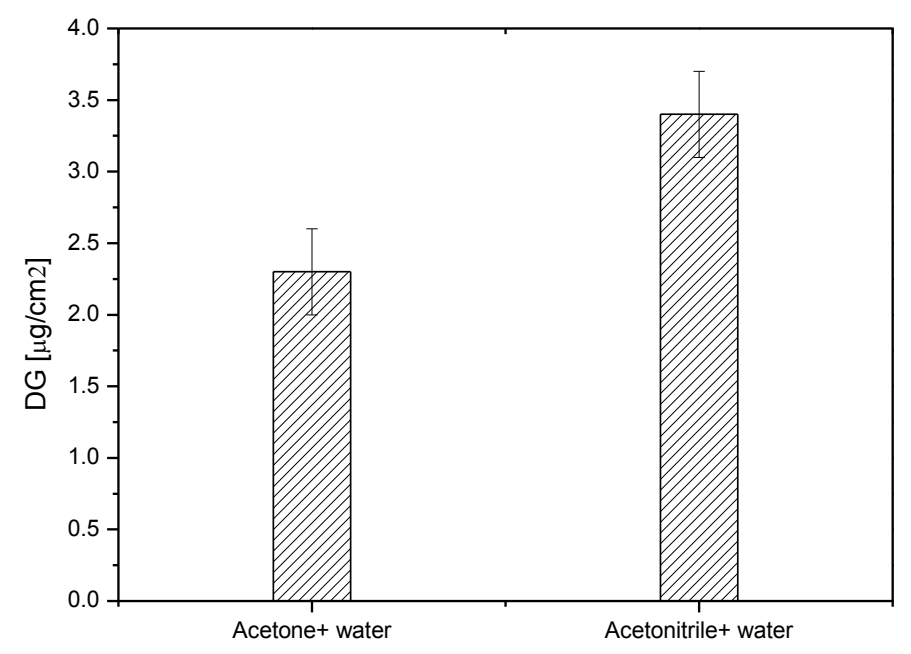

Figure 4.11 Degree of grafting for PtBMA on PET membrane surface versus the different solvents, acetone / water and $\mathrm{MeCN} /$ water solution mixture ( $\mathrm{V}: \mathrm{V}=9: 1)$, in $1.5 \mathrm{~mol} / \mathrm{L}$ tBMA with a catalyst system of $[t \mathrm{BMA}]:[\mathrm{CuCl}]:[\mathrm{Bpy}]=75: 1: 2$ at ambient temperature for $6 \mathrm{~h}$.

SI-ATRP of $t \mathrm{BMA}$ in MeCN aqueous solution has higher reactivity than that in acetone aqueous solution. This is due to the good wetting property of MeCN aqueous solution to PET material and the higher polarity of $\mathrm{MeCN}$ than that of acetone. At good wetting, more polar solution can accelerate the ATRP rate by quick transport of reagents to the membrane surface. The kinetics of SI ATRP of tBMA in MeCN aqueous solution was further characterized via a time series and addition of $\mathrm{Cu}(\mathrm{II})$ in the catalyst system (Figure 4.12). 


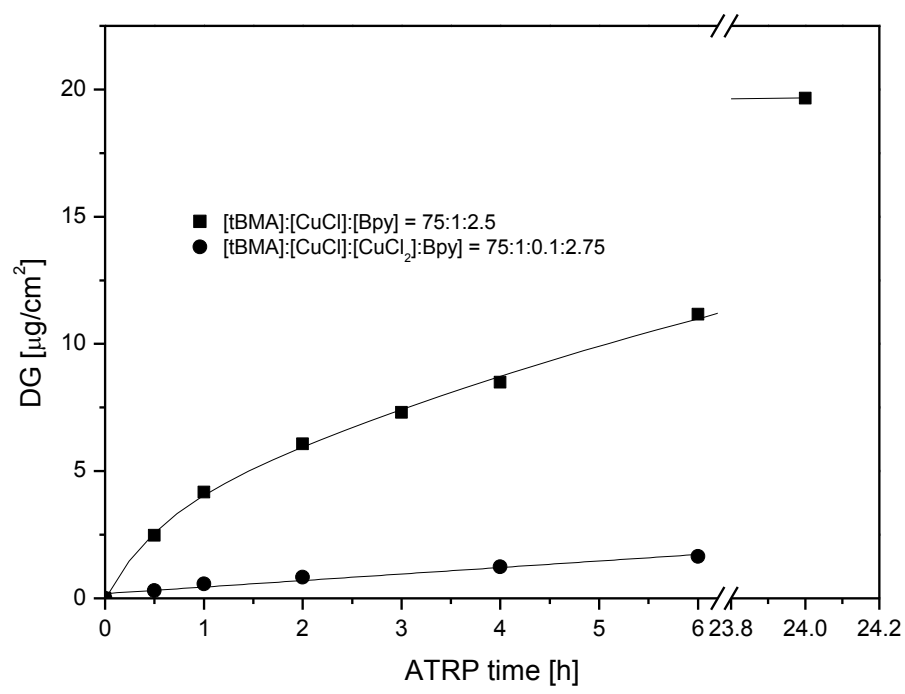

Figure 4.12 Degree of grafting for PtBMA on PET membrane surface versus ATRP reaction time in MeCN / water solution mixture $(\mathrm{V}: \mathrm{V}=9: 1)$ in $1.5 \mathrm{~mol} / \mathrm{L} t \mathrm{BMA}$ with a catalyst system

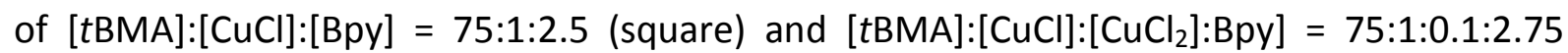
(point) at ambient temperature.

For $6 \mathrm{~h} \mathrm{SI-ATRP,} \mathrm{the} \mathrm{DG} \mathrm{is} 11.2 \mu \mathrm{g} / \mathrm{cm}^{2}$, which was much higher than the old maximum value $3.4 \mu \mathrm{g} / \mathrm{cm}^{2}$, this resulted from the newly bought copper (I) chloride. The old copper (I) chloride had green color and the new one was gray, the change of the color means the old copper(I) chloride has reacted in the air during the long term storage. This was confirmed by the elemental analysis.

Table 4.2 Content of elements from elemental analysis of copper(I) chloride.

\begin{tabular}{c|c|c|c}
\hline $\mathrm{CuCl}$ & Old sample & New sample & Theoretical \\
\hline $\mathrm{Cu}(\%)$ & 62.26 & 64.15 & 64.19 \\
\hline $\mathrm{Cl}(\%)$ & 30.3 & 34.16 & 35.80 \\
\hline $\mathrm{H}(\%)$ & 0.19 & 0 & 0 \\
\hline
\end{tabular}

Hydrogen atom was observed in the old copper (I) chloride by the elemental analysis, the cooper chloride reacted with water and oxygen in the air as follows (Scheme 4.2).

$$
6 \mathrm{CuCl}+3 / 2 \mathrm{O}_{2}+3 \mathrm{H}_{2} \mathrm{O} \rightarrow 2 \mathrm{Cu}_{3} \mathrm{Cl}_{2}(\mathrm{OH})_{4}+\mathrm{CuCl}_{2}
$$

Scheme 4.2 Oxidation of copper (I) chloride in air. 
The produced $\mathrm{CuCl}_{2}$ and $\mathrm{Cu}_{3} \mathrm{Cl}_{2}(\mathrm{OH})_{4}$ had green color and reduced the real reactive $\mathrm{CuCl}$ concentration. $\mathrm{CuCl}_{2}$ can increase the deactivation constant and reduce the reaction rate. Comparison with the theoretical value, the new received $\mathrm{CuCl}$ had high purity. In order avoid the reaction between $\mathrm{CuCl}$ and air, $\mathrm{CuCl}$ was treated by vacuum immediately and then protected with argon.

ATRP with new $\mathrm{CuCl}$ had a quick rate and due to the initial "jump" on the curve of DG versus reaction time, it was difficult to control the chain's growth on membrane surface very precisely. The addition of $\mathrm{CuCl}_{2}$ was tried to improve the control of the ATRP. The ATRP with well defined addition of $\mathrm{CuCl}_{2}$ had a perfect control on the surface grafting demonstrated by the linear relationship between DG and reaction time in Figure 4.12 and small error of grafted DG $0.04 \mu \mathrm{g} / \mathrm{cm}^{2}$ in Table 4.3. Adding $\mathrm{Cu}$ (II) decreased the radical concentration at the early stage of ATRP and using Bpy as ligand were useful to avoid the radical coupling reaction (initial "jump"), ${ }^{[137]}$ because $\mathrm{Cu}(\mathrm{II})$ could shift the reaction equilibrium to the left side. The reaction rate was reduced significantly, so control on the polymer growth is feasible via variation of the reaction time.

Table 4.3 Result of ATRP in $1.5 \mathrm{~mol} / \mathrm{L} t \mathrm{BMA}$ with a catalyst system of [tBMA]:[CuCl]: [CuCl 2$]$ : [Bpy] $=$ 75:1:0.1:2.75 at ambient temperature for $4 \mathrm{~h}$.

\begin{tabular}{c|c|c|c|c|c|c|c}
\hline Sa mple & 1 & 2 & 3 & 4 & 5 & MV & Error \\
\hline Al $[\mathrm{mg}]$ & 11.63 & 11.62 & 11.72 & 11.77 & 11.77 & 11.70 & 0.07 \\
\hline ATRP $[\mathrm{mg}]$ & 12.03 & 12.05 & 12.13 & 12.17 & 12.17 & 12.11 & 0.07 \\
\hline $\begin{array}{c}\text { ATRP-DG } \\
{\left[\mu \mathrm{g} / \mathrm{cm}^{2}\right]}\end{array}$ & 2.06 & 2.01 & 2.11 & 2.06 & 2.06 & 2.06 & 0.04 \\
\hline
\end{tabular}

For 3D model of protein adsorption via grafted polymers, the polymer chain should have an appropriate chain length regarding to the size of the proteins. Protein adsorption by too long chain prefers in a circle round form, this will lead to an elution problem after protein imprinting (cf. Figure 1.1). In contrast, protein can not penetrate into a very thin grafted polymer layer and has 2D interaction with the grafted polymer. This leads to a partial surface imprinting of protein and normally results in a poor imprinting property. For two-step grafting, control on the grafted polymer chain length via ATRP is the fundamental and key step for successful protein imprinting. 


\subsection{Hydrolysis of poly(tert.-butyl methacrylate) (PtBMA) to poly-(methacrylic acid) (PMAA) on PET membrane surface}

SI-ATRP for PMAA grafting faced the problem that the acid monomer tends to form a complex with copper based catalyst to yield ineffective carboxylate. ${ }^{[56]}$ An alternative to obtain PMAA is synthesis of PtBMA and subsequent hydrolysis of the tert-butyl ester protective group. After surface grafting of PtBMA on PET surface (cf. section 4.3), its hydrolysis was first tested in methansulfonic acid chloromethane solution at room temperature (Figure 4.13). ${ }^{[65,71]}$

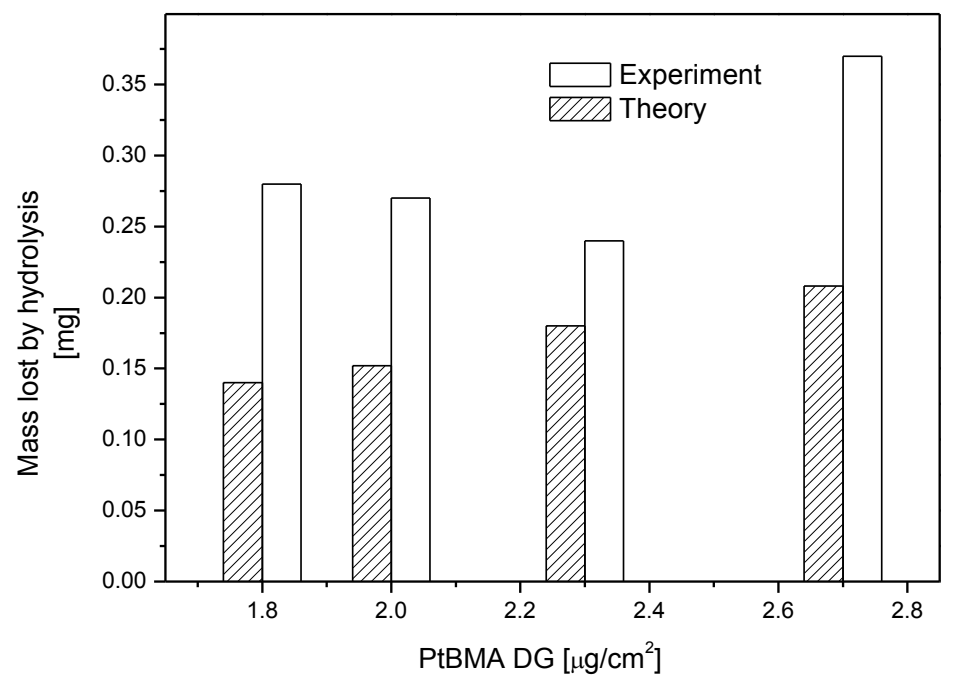

Figure 4.13 Mass loss by hydrolysis of PtBMA to PMAA on PET membrane versus DG of PtBMA. The hydrolysis was taken placed in dichloromethane containing $1 \%$ methansulfonic acid at room temperature for $15 \mathrm{~min}$. Open columns represent experimental results and open ones represent the theoretical values.

All the experimental mass losses were higher than the theoretical values. Not only the tert butyl groups were hydrolyzed, the methansulfonic acid is so strong (pKa= -2.6 in water) and could also hydrolyze the ester bonds of PET substrate. The leakage of the backbone chain fragments of PET resulted in partial removal of the grafted polymer chains on the surface and led to a higher mass loss after hydrolysis than the theoretical value. The stability of PET in polar organic solvents is another influence factor which will be discussed later. The hydrolysis kinetics of the tert butyl ester group in methansulfonic acid was investigated by Müller et al. via observation the change of polymer layer thickness and contact angle. The 
hydrolysis was completed inner $5 \mathrm{~min}$, so short reaction time is difficult to control the hydrolysis on membrane surface. ${ }^{[138]}$

After hydrolysis, the ability of PMAA binding to Lyz was tested in a $\mathrm{pH}=7$ phosphate buffer, when PMAA is negatively charged $(\mathrm{pKa} \approx 4.65)$ and Lyz with the isoelectric point about 11 has a positive net charge (Figure 4.14).

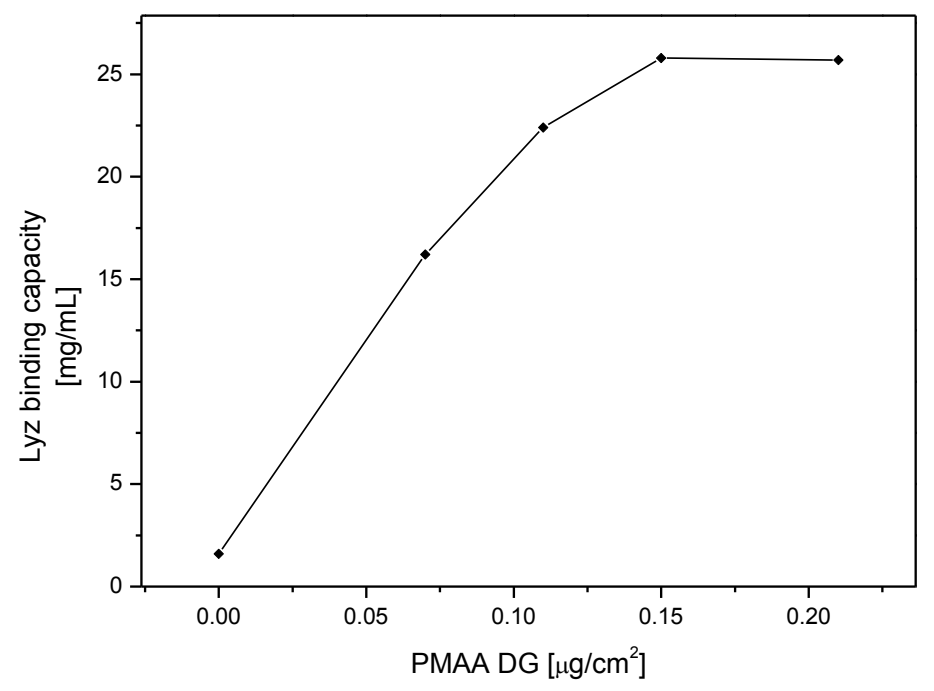

Figure 4.14 Lysozyme binding capacity versus the DG of PMAA, the DG = 0 was the PtBMA-gmembrane without hydrolysis. Protein adsorption condition: $2.0 \mathrm{~g} / \mathrm{L}$ Lyz in PBS $(\mathrm{pH}=7, \mathrm{C}=$ $0.066 \mathrm{~mol} / \mathrm{L})$, room temperature, $24 \mathrm{~h}$.

In comparing with the PtBMA, PMAA had much higher Lyz adsorption capacity. The domination of electrostatic interaction over other interactions, such as hydrophobic interaction between protein and grafted polymer, provided important information to design the MIP and subsequent template elution in the future. Even the hydrolysis condition was not perfect, the capacity of Lyz binding is still proportional to the DG of PMAA. It was feasible to control the Lyz adsorption by ATRP of PtBMA, when hydrolysis condition is appropriate and just tert butyl ester group is hydrolyzed, bcause the precise control of DG PtBMA has been obtained via the ATRP time in Figure 4.12.

To reduce the strength of hydrolysis condition, TFA ( $p K a=-0.3$ ) was used, which is weaker than methansulfonic acid. At first, the stability of base PET membrane was tested in different organic solvents containing 1\% TFA (Figure 4.15). 


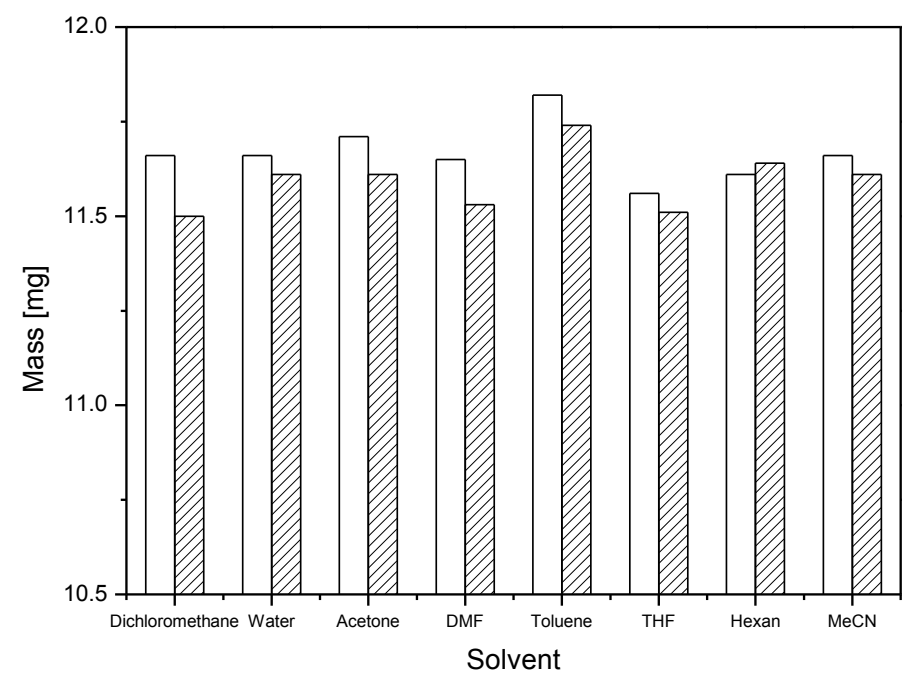

Figure 4.15 Mass of PET base membranes versus different solvents, open columns are the original membrane mass and solid columns are the mass after $24 \mathrm{~h}$ soaking with shaking 120 rmp at room temperature.

The PET membrane was less stable in dichloromethane, acetone, DMF, and toluene than in water, THF and MeCN. The mass of membrane apparently increased in hexane which may resulted from weighing error. Through calculation of the mass loss ratio, water and MeCN were the best stable solvents and chosen for the further optimization. The stability of PtBMA-g-PET membrane was also tested in the same condition and no mass loss has been observed. In order to accelerate the hydrolysis, volume ratio of TFA was increased from $5 \%$ to $50 \%$ at $50{ }^{\circ} \mathrm{C}$. First of all, the stability of the base membrane was confirmed again in the new conditions (Figure 4.16). 


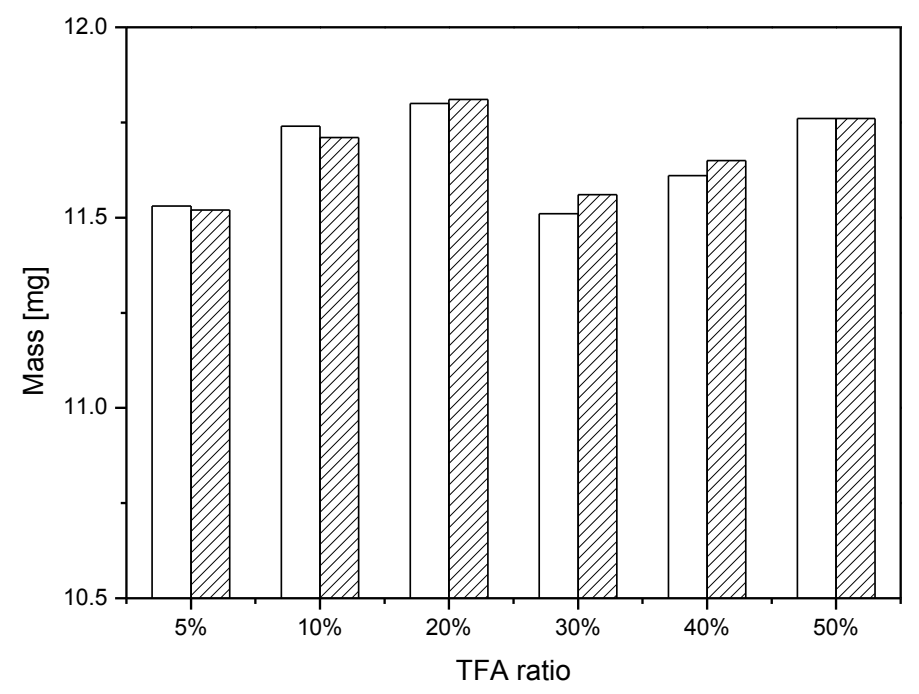

Figure 4.16 Mass loss of PET basis membranes versus the volume ratio of TFA in MeCN, open columns are the original membrane mass and solid columns are the mass after $24 \mathrm{~h}$ soak with shaking $120 \mathrm{rpm}$ at $50^{\circ} \mathrm{C}$.

The PET membrane was still stable in 50\% TFA solution which was applied in the further hydrolysis. Grafted PtBMA on membrane surface with DG $=2.6 \mu \mathrm{g} / \mathrm{cm}^{2}$ was hydrolyzed under different conditions (Figure 4.17). For $20 \mathrm{~h}$, only 33\% mass was lost by hydrolysis, which was smaller than theoretical value $40 \%$. To increase the hydrolysis effect, the hydrolysis in water was investigated with the similar result of $32 \%$ mass loss. This is due to the poor solubility of the tert butyl group in water. In consideration of the surface wetting property and solubility of the split tert butyl agent, a solvent of mixture of water and $\mathrm{MeCN}$ was with volume ratio 1:1 was tested and the reaction time was extended in favor of hydrolysis. For $20 \mathrm{~h}$, the percent of mass loss of $41 \%$ was near to theoretical value $40 \%$, increasing the hydrolysis time to $24 \mathrm{~h}$, the mass loss percent reached $53 \%$ which resulted from the hydrolysis of PET backbone chain and led to loss of grafted polymer chain. $50 \%$ TFA in mixture of MeCN / water $(\mathrm{V}: \mathrm{V}=1: 1)$ at $50^{\circ} \mathrm{C}$ and time $20 \mathrm{~h}$ was chosen for the later hydrolysis. 


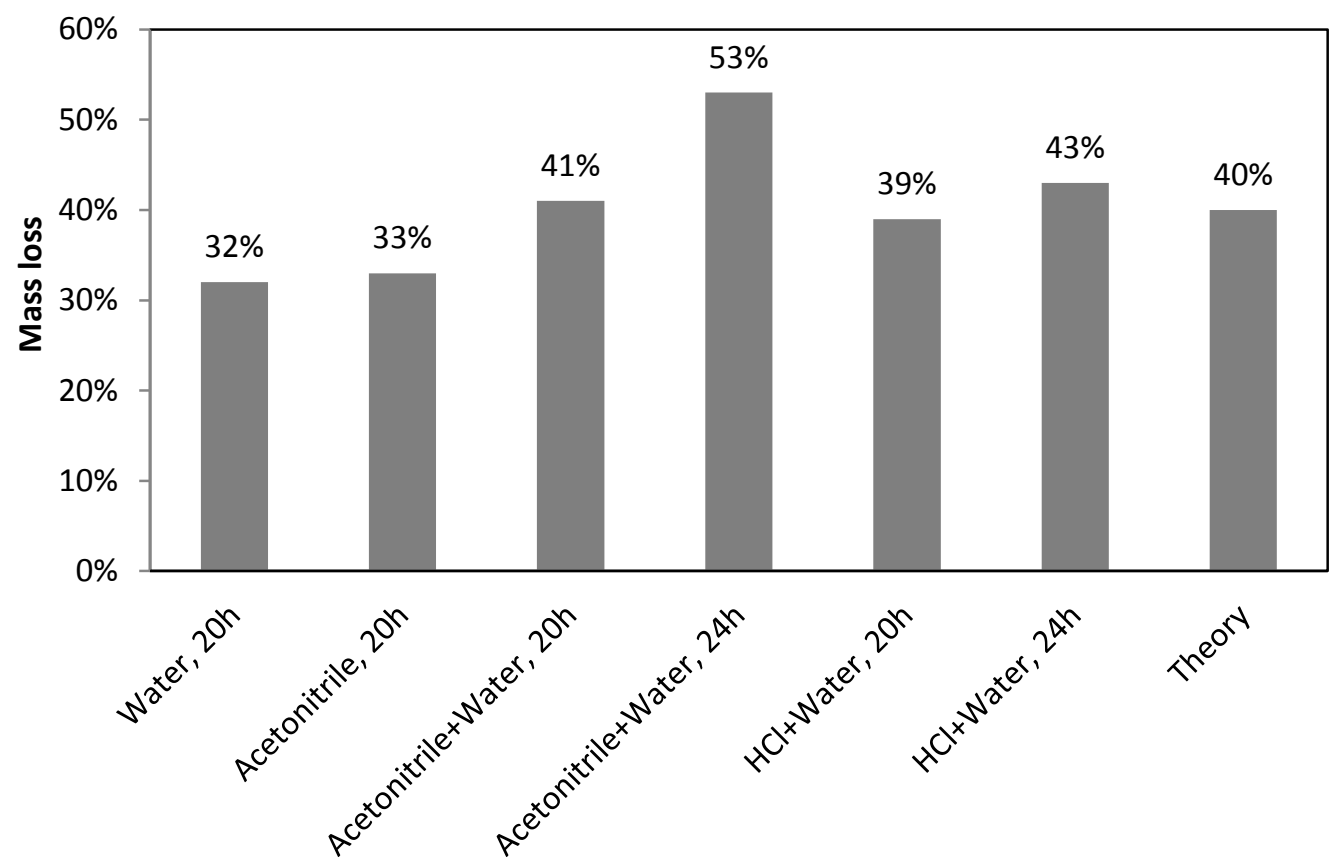

Figure 4.17 Mass loss percent of grafted polymer by hydrolysis from PtBMA (DG $=2.6$ $\mu \mathrm{g} / \mathrm{cm}^{2}$ ) to PMAA on PET membranes in 50\% TFA solution versus the conditions in form "solvent, time".

$\mathrm{HCl}$ was later also used as acid for hydrolysis of PtBMA to PMAA in pure water, which showed good performance similar to the TFA method, even better for $24 \mathrm{~h}$ reaction time.

The hydrolysis condition was investigated by hydrolysis of the time series samples till $4 \mathrm{~h}$ ATRP with increased DG of PtBMA to $2.0 \mu \mathrm{g} / \mathrm{cm}^{2}$, which was less than the DG of the samples for hydrolysis optimization. So the hydrolysis time was reduced to $18 \mathrm{~h}$ to minimize the corrosion of the base membrane (Figure 4.18). 


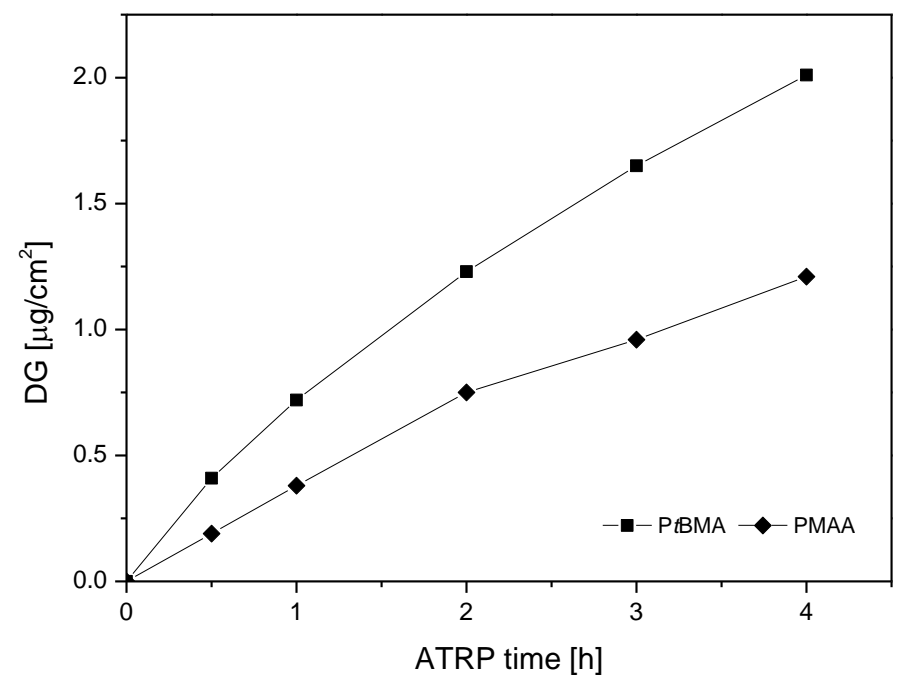

Figure 4.18 Degree of grafting for PtBMA on PET membrane surface (squares) versus the reaction time of ATRP in $1.5 \mathrm{~mol} / \mathrm{L} t \mathrm{BMA}$ solution in $\mathrm{MeCN} /$ water solution mixture $(\mathrm{V}: \mathrm{V}=$ 9:1) with a catalyst system of $[$ tBMA]:[CuCl]:[CuCl$]$ :[Bpy] $=75: 1: 0.1: 2.75$ at ambient temperature; PET-g-PMAA (rhombuses) was obtained via hydrolysis of the PET-g-PtBMA membranes in 50\% TFA solution in MeCN / water mixture $(\mathrm{V}: \mathrm{V}=1: 1)$ at $50^{\circ} \mathrm{C}$ for $18 \mathrm{~h}$.

After hydrolysis, the the linear relationship between ATRP time and DG was preserved very well. The experimental ratio $D_{G_{P M A A}} / D_{G_{P t B M A}}$ was about 0.56 which was close to the theoretical value $\left(M_{w, P M A A} / M_{w, P t B M A}=0.60\right)$. This is an additional proof for the selective, quantitative hydrolysis of PtBMA to PMAA. Consequently, as expected for a controlled polymerization of MAA, variation of ATRP reaction time and subsequent suitable hydrolysis seem to be satisfactory to control PMAA grafting on PET membrane surface.

After hydrolysis, surface group was converted from hydrophobic tert.-butyl group to hydrophilic acid group. Because the hydrophilicity of the membrane surface changed significantly after every step (cf. Schemes $\mathbf{1 . 1}$ and 1.2), modifications on membrane surface were characterized via contact angle (Figure 4.19). The contact angle increased from $56^{\circ}$ to $94^{\circ}$ after ATRP initiator acryl bromide immobilization. After coverage with PtBMA, containing tert.-butyl groups in a high density, the surface of the membrane became even more hydrophobic. The contact angle reduced largely from $110^{\circ}$ to $38^{\circ}$ after the hydrolysis of PtBMA to PMAA. This is at least a strong qualitative proof that the synthesis of the scaffold PMAA had been successful. 


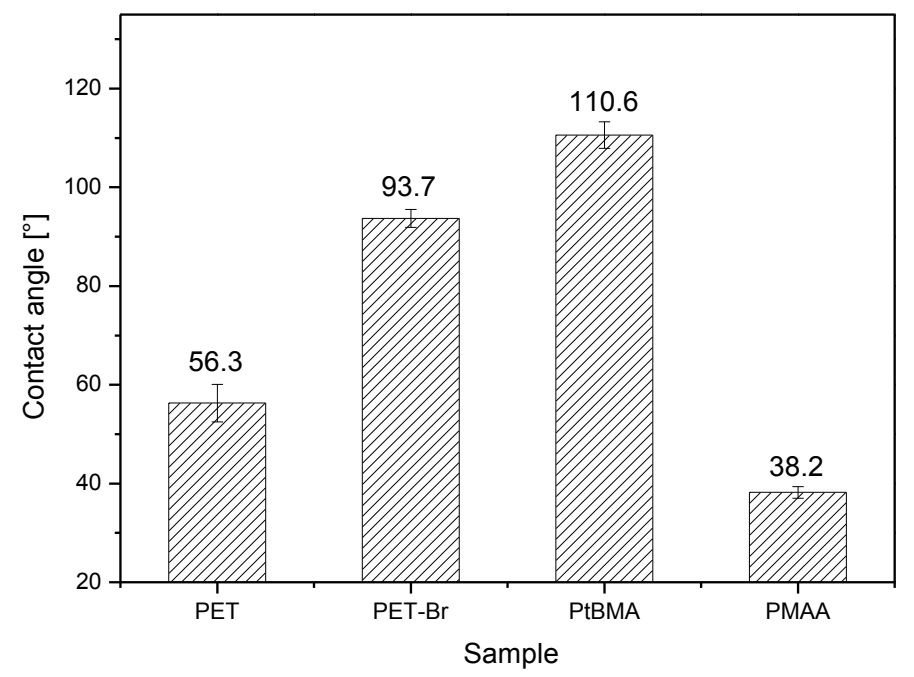

Figure 4.19 Contact angle results for membranes after different stages of functionalization. PET-Br corresponds to a membrane after ATRP initiator immobilization; PtBMA and PMAA correspond to the membranes grafted with PtBMA (4 h ATRP; $2.26 \mu \mathrm{g} / \mathrm{cm}^{2}$ ) and PMAA (1.17 $\left.\mu \mathrm{g} / \mathrm{cm}^{2}\right)$, respectively.

Pore size change of the tracked etched membrane is useful to characterize the surface grafting and calculated the dry polymer layer thickness.

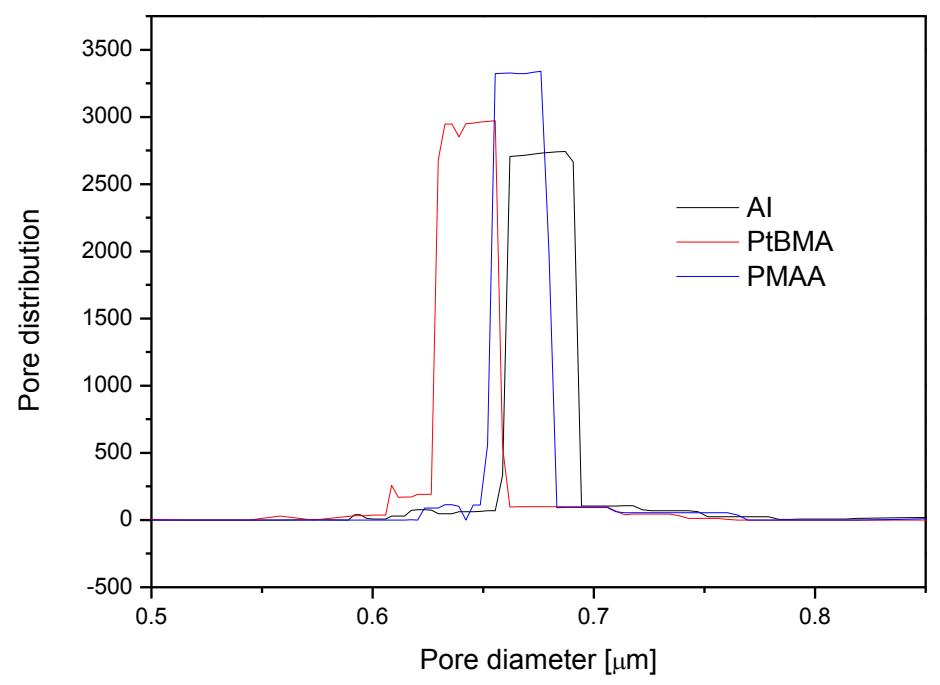

Figure 4.20 Pore distribution versus the pore diameter of PET membranes: $\mathrm{Al}$ is the membrane after ATRP initiator immobilization PtBMA and PMAA correspond to the 
membranes grafted with PtBMA (2 h ATRP; $\left.0.57 \mu \mathrm{g} / \mathrm{cm}^{2}\right)$ and PMAA $\left(0.36 \mu \mathrm{g} / \mathrm{cm}^{2}\right)$, respectively.

After Al immobilization, the average pore diameter is $679 \mathrm{~nm}$. The diameter was reduced by surface grafting of the PtBMA to $645 \mathrm{~nm}$ via $2 \mathrm{~h}$ ATRP with DG $0.57 \mu \mathrm{g} / \mathrm{cm}^{2}$, and the grafted layer thickness in dry state (or fully collapsed) is $17 \mathrm{~nm}$. After hydrolysis, the big side tert.butyl group was removed, the main grafted polymer chain filled the new free space via collapse in dry state. After hydrolysis, the pore diameter increased from $645 \mathrm{~nm}$ to $669 \mathrm{~nm}$, the new PMAA lay had a thickness of $5 \mathrm{~nm}$. The distribution of pore size has not changed significantly via SI ATRP and subsequent hydrolysis, the isotropic pore structure was preserved and the Hagen-Poiseuille theory is applicable to calculate hydrodynamic layer thicknesses of grafted PtBMA and PMAA.

After hydrolysis the permeability of PMAA-g-PET membrane was measured with different liquids to investigate the conformation of the grafted polyelectrolyte PMAA and characterize it via calculation of hydrodynamic layer thickness (Figure 4.21).

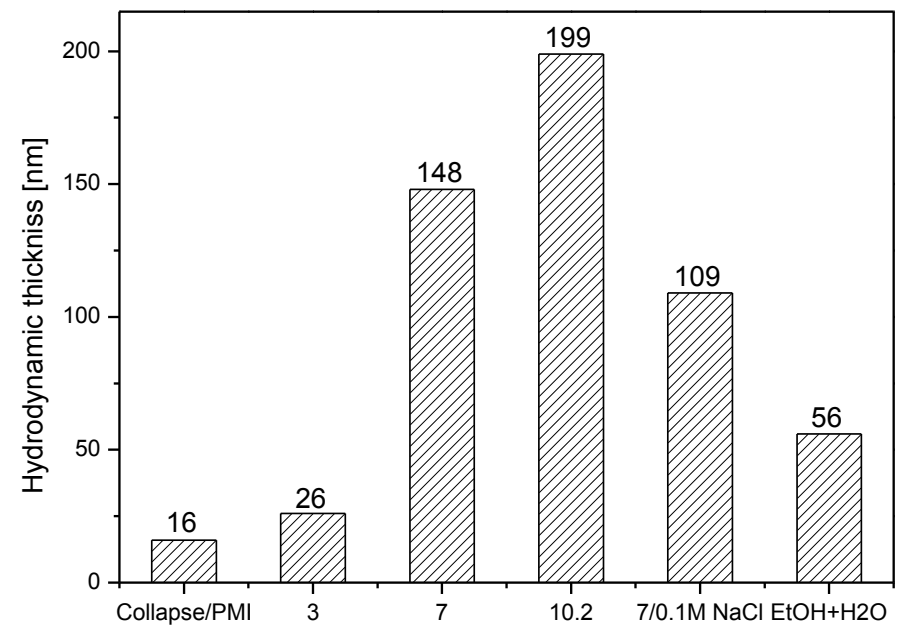

Figure 4.21 Hydrodynamic layer thickness of grafted PMAA on PET pore wall with DG 0.74 $\mu \mathrm{g} / \mathrm{cm}^{2}$ in different solutions. "Collapse" result represents the dry layer thickness measured by PMI (cf. Figure 4.20), pH varied with the values 3, 7, and 10.2. Addition of $\mathrm{NaCl}$ in PBS (pH $=7,0.1 \mathrm{~mol} / \mathrm{L})$ and mixture of water and ethanol $(\mathrm{pH}=7.2)$ were also to investigated the polyelectrolyte's conformation. 
Original permeate is PBS $(5 \mathrm{mmol} / \mathrm{L}, \mathrm{pH}=7)$ and $\mathrm{pH}$ value was calibrated to acid $(\mathrm{pH}=3)$ and basic $(\mathrm{pH}=10.2)$ range, with $\mathrm{HCl}$ and $\mathrm{NaOH}$, respectively. Because pKa of PMAA is about 4.65, the carboxyl groups of PMAA chains were not deprotonated in PBS with $\mathrm{pH}=3.0$, the hydroydynamic layer thickness of $26 \mathrm{~nm}$ was only a little thicker than the $16 \mathrm{~nm}$ dry layer thickness from PMI measurement. For increasing $\mathrm{pH}$, the conformation got more and more outstretched with the hydrodynamic layer thickness increasing from $26 \mathrm{~nm}$ to $199 \mathrm{~nm}$. Because more and more carboxyl groups are deprotonated with negative charge and repel each other when the $\mathrm{pH}$ increased from 3 to 10.2. Addition of salt in PBS with $\mathrm{pH}=7$ led a lower hydrodynamic layer thickness $109 \mathrm{~nm}$, while it is $148 \mathrm{~nm}$ in PBS without added salt. Because the electrostatic repulsion between the $\mathrm{COO}^{-}$groups were shield by the $\mathrm{Na}^{+}$and the swelling of the polymer chain was reduced. The carboxyl group can be depronated more easily in pure water than in the mixture of water and ethanol, this led to the small hydrodynamic layer thickness of $56 \mathrm{~nm}$ in ethanol aqueous solution than in pure water. And the solubility of $\mathrm{PMAA}^{-}$is also lower in ethanol compared to water. Even the $\mathrm{pH}=7.2$ of the mixture of water and ethanol is little higher than 7.0 of the PBS. The change of PMAA conformation is illustrated in Figure 4.22.

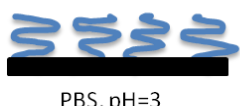

PBS, $\mathrm{pH}=3$

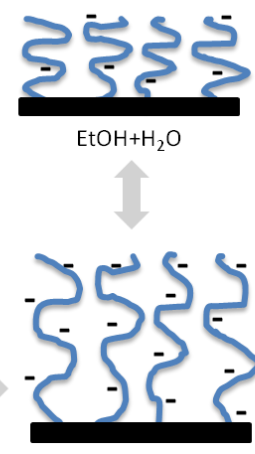

PBS, $\mathrm{pH}=7$

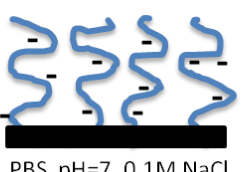

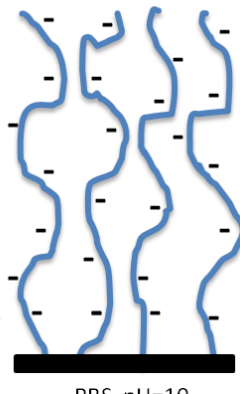

$\mathrm{PBS}, \mathrm{pH}=10$

Figure 4.22 Schematic representation of the PMAA chain's conformation on PET surface in PBS as a function of $\mathrm{pH}$ and salt concentration; effect on the deprotonation of carboxyl group by mixing of organic ethanol into pure water. 
As Kato et al. proposed, ${ }^{[134]}$ the orientation of the grafted polymer with a stretched conformation in vertical direction to the membrane surface will be facilitated in brush regime. During the adsorption process, protein can penetrate to the deep position of the grafted polymer layer and become surrounded by multiple binding sites like in a 3D solution. This leads to a higher binding affinity. After brush grafting with polymers, the simple surface interaction (2D) between membrane and protein was switched to 3D interaction. ${ }^{[12]}$ 


\subsection{Optimization of UV-grafting/crosslinking on PET- $g$-PMAA membrane}

AAm is one of most popular monomers in protein imprinting systems. The PAAm hydrogel is biocompatible and neutral, and these factors were considered to reduce the non-specific interaction between PAAm and the proteins. For the two-step grafting strategy, the second grafting/crosslinking step is achieved via photo initiation of the ATRP acyl bromide initiator to form PAAm gel to fix the complex of scaffold PMAA and Lyz. In order to control the PAAm gel growth, the reactivity of AAm was investigated via variation of the UV-irradiation time, UV-intensity, and monomer concentration.

\subsubsection{Optimization of UV-irradiation time}

After hydrolysis, the reactivity of bromide on the PMAA-g-PET membrane surface was first characterized via UV-grafting/crosslinking in $0.7 \mathrm{~mol} / \mathrm{L}$ AAm and MBAA (molar ratio AAm:MBAA $=9: 1$ ) in water with $\mathrm{pH}=7.0$, which is similar to the condition for later Lyz adsorption (Figure 4.23).

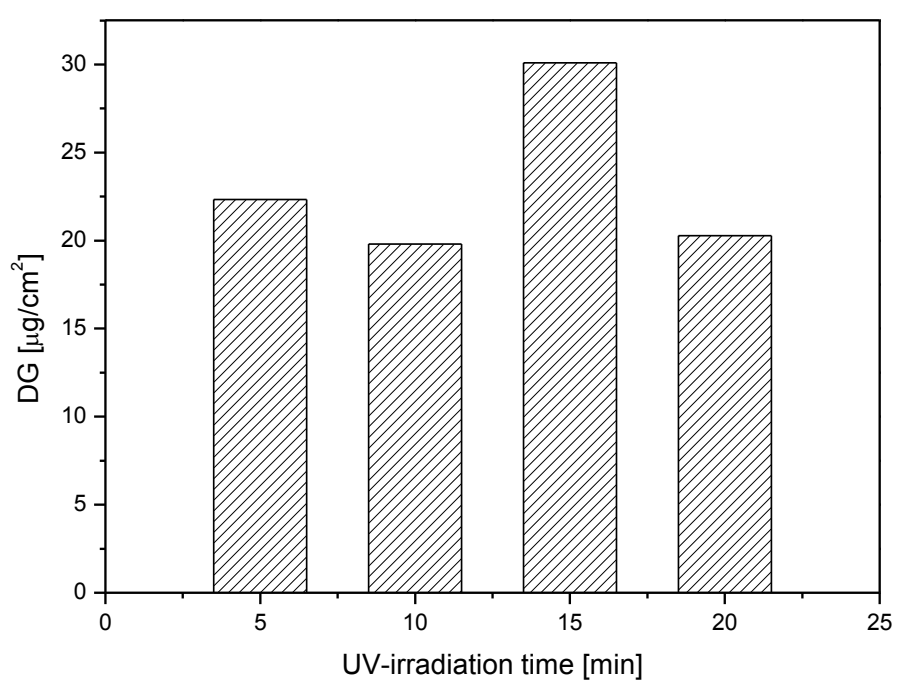

Figure 4.23 Influence of UV-irradiation time on the DG of grafted crosslinked PAAm on PET$g$-PMAA membrane surface $\left(D_{\text {PMAA }}=1.31 \mu \mathrm{g} / \mathrm{cm}^{2}\right): 0.7 \mathrm{~mol} / \mathrm{L}$ UV-functional monomer AAm and MBAA with molar ratio $\mathrm{n}_{\mathrm{AAm}}: \mathrm{n}_{\mathrm{MBAA}}=9: 1$, i. e. degree of crosslinking $10 \%$, UV-intensity about $72 \mathrm{~mW} / \mathrm{cm}^{2}$. 
The DG of grafted PAAm on membrane surface was very high even more the $20 \mu \mathrm{g} / \mathrm{cm}^{2}$ by 5 min and $15 \mathrm{~min}$. In comparing with the DG from ATRP and subsequent hydrolysis of PtBMA to PMAA, it was not necessary to graft too much PAAm gel on the surface, this would lead to fully bury of imprinted Lyz (diameter about $4 \mathrm{~nm}$ ) imprinting via scaffold PMAA chain with a chain length less than $150 \mathrm{~nm}$. No clear tendency of DG versus irradiation time has been observed in Figure 4.23, the control of UV-grafting/crosslinking was poor under strong UVintensity $72 \mathrm{~mW} / \mathrm{cm}^{2}$ in $0.7 \mathrm{~mol} / \mathrm{L}$ UV-functional monomer AAm and MBAA. In order to reduce the PAAm content and improve the control of the UV-grafting/crosslinking, the UVgrafting/crosslinking was performed in the same reaction solution but under a lower UVintensity $24 \mathrm{~mW} / \mathrm{cm}^{2}$, also in respect to reduce the DG of PAAm (Figure 4.24).

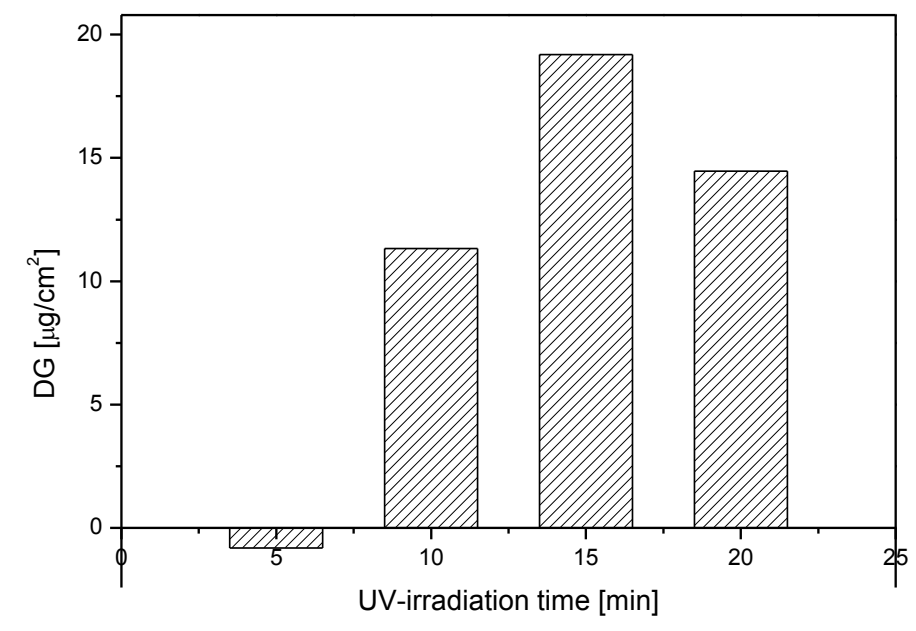

Figure 4.24 Influence of UV-irradiation time on the DG of grafted crosslinked PAAm on PET$g$-PMAA membrane surface $\left(D_{\text {PMAA }}=1.31 \mu \mathrm{g} / \mathrm{cm}^{2}\right): 0.7 \mathrm{~mol} / \mathrm{L}$ UV-fuctional monomer AAm and MBAA with mol ratio $\mathrm{n}_{\mathrm{AAm}}: \mathrm{n}_{\mathrm{MBAA}}=9: 1$, i.e. degree of crosslinking $10 \%$, UV-intensity about $24 \mathrm{~mW} / \mathrm{cm}^{2}$.

Till 15 min irradiation time, the DG of PAAm has an increased tendency under lower UVintensity of $24 \mathrm{~mW} / \mathrm{cm}^{2}$. For $5 \mathrm{~min}$ irradiation time, the DG was negative resulting from consumption of produced radical by oxygen in the reaction solution to form no reactive peroxyl radical and split of the bromide from the membrane surface. The DG jumped to a high value of more the $10 \mu \mathrm{g} / \mathrm{cm}^{2}$ from $5 \mathrm{~min}$ to $10 \mathrm{~min}$, which was still much higher than the requisite appropriate value. Reduction of the UV-intensity has improved the control of 
the UV-grafting/crosslinking, but it was still not enough. The lower UV-intensity $24 \mathrm{~mW} / \mathrm{cm}^{2}$ was chosen for the further optimization.

\subsubsection{Optimization of monomer concentration}

The reduction of the monomer concentration was tested to improve the control of UVgrafting/crosslinking and meanwhile to reduce the DG of PAAm under UV-irradiation 24 $\mathrm{mW} / \mathrm{cm}^{2}$ for $10 \mathrm{~min}$.

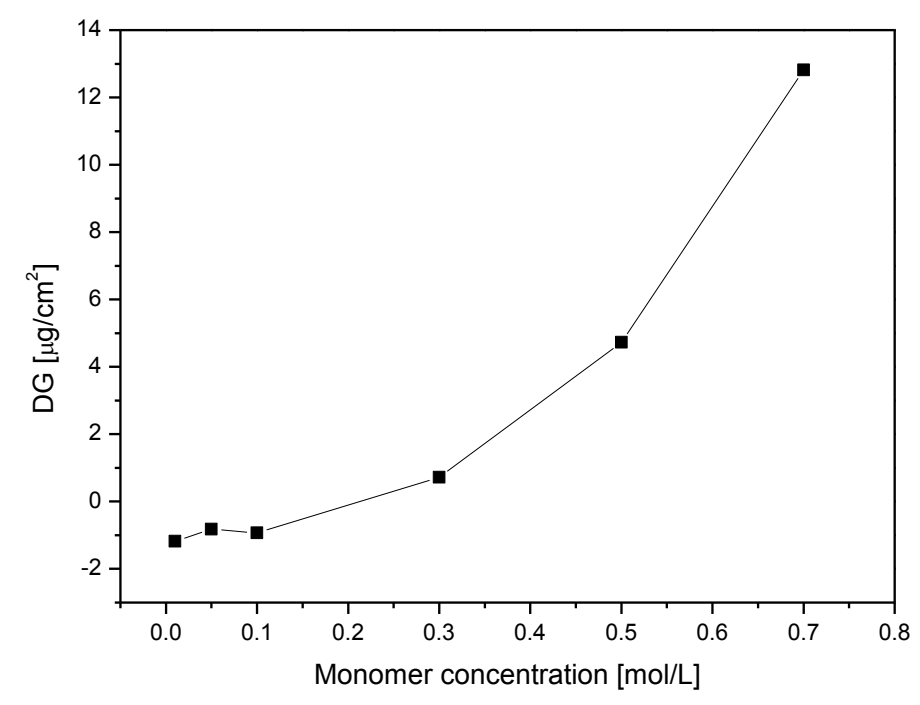

Figure 4.25 Degree of grafting for PAAm on PET-g-PMAA membrane surface $\left(D G_{P M A A}=1.43 \mu \mathrm{g} / \mathrm{cm}^{2}\right)$ versus the concentration of the functional monomer AAm and MBAA with degree of crosslinking $10 \%$, irradiation under the UV-intensity $24 \mathrm{~mW} / \mathrm{cm}^{2}$ for $10 \mathrm{~min}$.

Increase of monomer concentration led to an increasing tendency of PAAm DG. When the monomer concentrations were small (till $0.1 \mathrm{~mol} / \mathrm{L}$ ), the DGs of PAAm were negative, which was due to the small monomer addition rate and loss of bromide after cleavage of $\mathrm{C}-\mathrm{Br}$ bond by UV-light at lower concentration. As discussed above, the radicals formed by irradiation reacted with oxygen or combined at the low monomer concentration. Monomer concentrations of 0.5 and $0.7 \mathrm{~mol} / \mathrm{L}$ still led to much higher DG of PAAm than the range of PMAA when the time of SI-ATRP was less than $4 \mathrm{~h}$ (cf. Figure 4.13). 


\subsubsection{Optimization of UV-irradiation time with $0.3 \mathrm{~mol} / \mathrm{L}$ monomer concentration}

A monomer concentration of $0.3 \mathrm{~mol} / \mathrm{L}$ was chosen to further optimize the DG of PAAm gel via variation of irradiation time in order to obtain a DG range of PAAm based gel similar to the PMAA via ATPR for $4 \mathrm{~h}$ and subsequent hydrolysis.

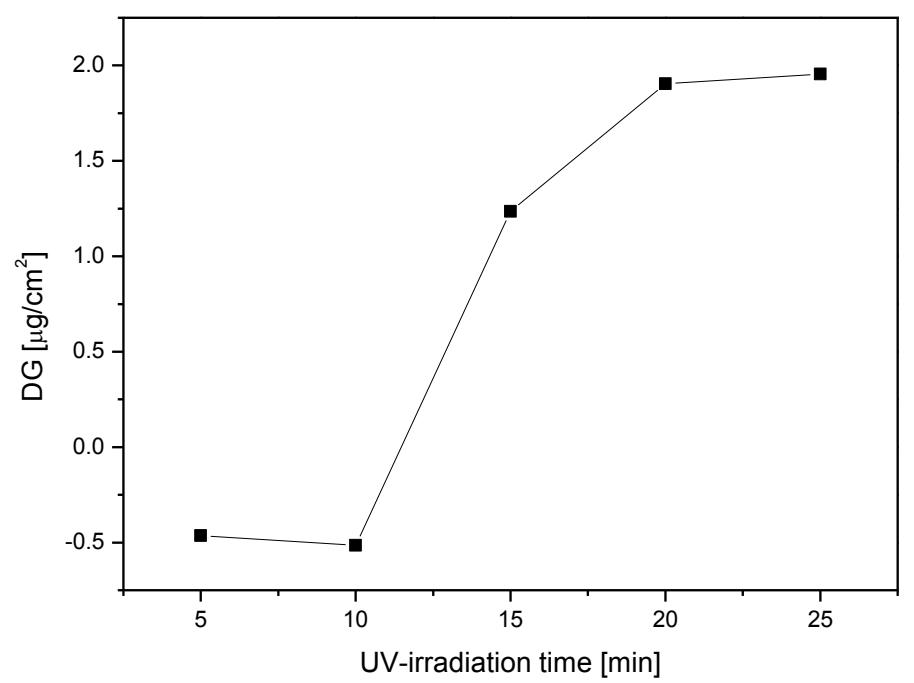

Figure 4.26 Degree of grafting for PAAm on PET-g-PMAA membrane surface $\left(\right.$ DG $_{\mathrm{PMAA}}=1.43$ $\mu \mathrm{g} / \mathrm{cm}^{2}$ ) versus the irradiation time: $0.3 \mathrm{~mol} / \mathrm{L}$ AAm and MBAA with degree of crosslinking $10 \%$, irradiation under the UV-intensity $24 \mathrm{~mW} / \mathrm{cm}^{2}$.

The negative DG after short time irradiation of 5 and 10 min was still observed. From 10 min to $25 \mathrm{~min}$ irradiation time, the rate of UV-grafting/crosslinking was reduced. This was different to the UV-grafting without crosslinker because the crosslinker combined part of the propagating radicals. Without crosslinker, the propagating radicals were normally terminated by the bromide radical and could be later reversibly activated by the UV-light, this is similar to the living radical polymerization and resulted in an almost linear relationship between irradiation time and degree of grafting (Figure 4.27). The UV-irradiation time from 10 to 25 min provided a suitable DG range of PAAm from 0 to $2 \mu \mathrm{g} / \mathrm{cm}^{2}$ and was chosen for

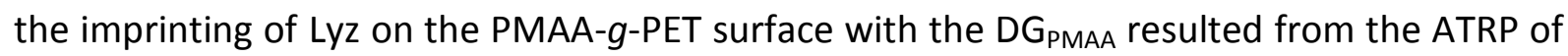
tBMA till $4 \mathrm{~h}$ and subsequent hydrolysis. According to the MIP preparation scheme (cf. Figure 1.1), the surface conformation and property will be changed after protein adsorption, which may lead to the change of the bromide reactivity on the membrane surface, but the above optimizations can still provide useful information for the further optimization of the 
UV-grafting/crossling for imprinting (similar to interpenetrating polymerization) which mainly decided the performance of the imprinted structure. ${ }^{[1]}$

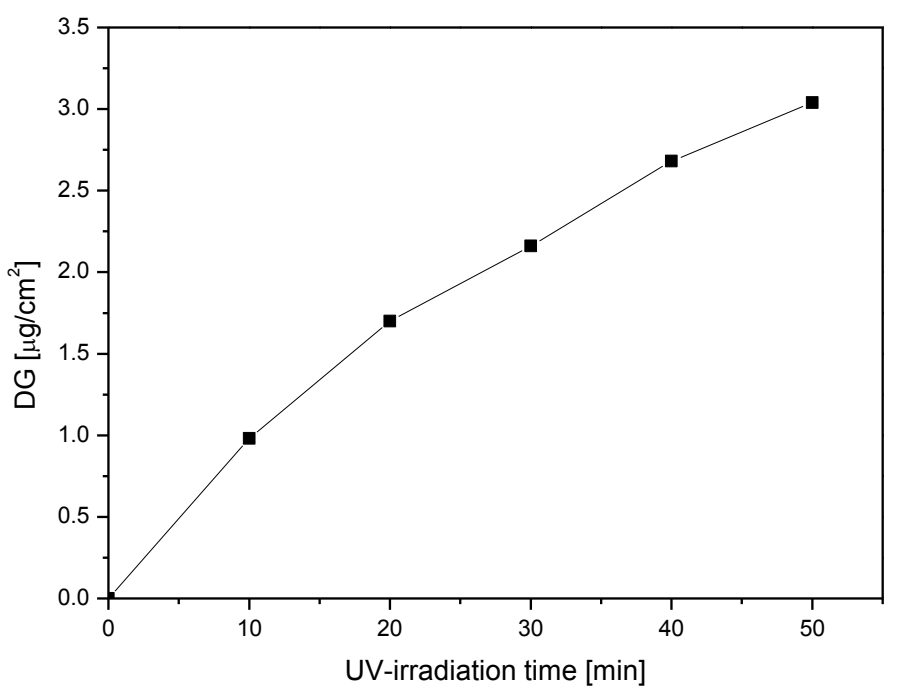

Figure 4.27 Degree of grafting for PAAm on Al immobilized membrane surface versus the irradiation time: $0.7 \mathrm{~mol} / \mathrm{L} A A m$, irradiation under the UV-intensity $24 \mathrm{~mW} / \mathrm{cm}^{2}$. 


\subsection{Lysozyme imprinting on PET membrane}

Lyz is an enzyme and a part of the innate immune system, which lyses certain bacteria by hydrolyzing the ß-linkages between the muramic acid and $\mathrm{N}$-acetylglucosamine of the mucopolysaccharides which are present in the bacterial cell wall. Lyz is applied generally as a cell disrupting agent for extraction of bacterial intracellular products, such as an antibacterial agent in ophthalmologic preparation, as food additive in milk products, and as a drug for treatment of ulcers and infections. Under the growing commercial pressure to reduce processing costs of Lyz purification, more efficient, simple, and relatively less expensive separation techniques were developed, the synthetic polymer based on molecular imprinting is one of the potential rewarding techniques. ${ }^{[139]}$

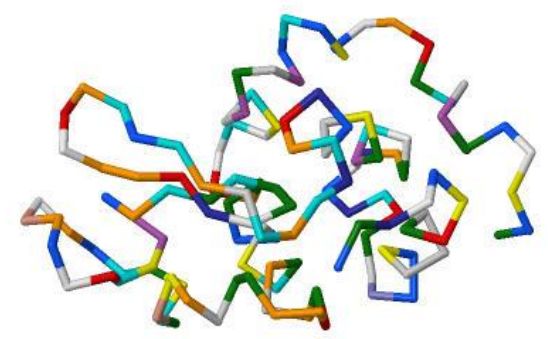

Figure 4.28 Structure of Lyz colored by amino acid (PDB ID: 2LYZ). ${ }^{[140]}$

CyC with similar MW and isoelectric point relative to Lyz was used for the competitive adsorption to characterize the property of the Lyz imprinted structure (Table 4.4).

Table 4.4 Information on Lyz and CyC.

\begin{tabular}{c|c|c}
\hline & Lyz & CyC \\
\hline MW $[\mathrm{kDa}]$ & $\sim 11.4$ & $\sim 12.6$ \\
\hline $\mathrm{pl}$ & $\sim 11.0$ & $\sim 10.2$ \\
\hline Size $\left[\mathrm{nm}^{3}\right]^{[141]}$ & $1.9 * 2.5^{*} 4.3$ & $2.6 * 3.2 * 3.3$ \\
\hline
\end{tabular}

\subsubsection{Hydrodynamic thickness of grafted layers}

For 3D model for protein adsorption and the second grafting/crosslinking step for protein imprinting, polymer layer thickness for protein imprinting is a key issue, too thick polymer layer leads more entrapment of protein and reduce the performance of the imprinted structure. Permeability measurements were carried out to investigate the thickness of the grafted layer and the conformation of the grafted polymer by using a mixture of ethanol and water with the volume ratio of $1: 1$ at $\mathrm{pH}=7.2$. The conformation of polymer in this state was 
similar to that during the protein adsorption. This solvent mixture was used to minimize the influence of the significantly different surface properties after different stages of the functionalization (cf. Sheme 1.1 and 1.2) onto pore wetting. The hydrodynamic layer thickness was calculated from permeability and results are shown in Figure 4.29.

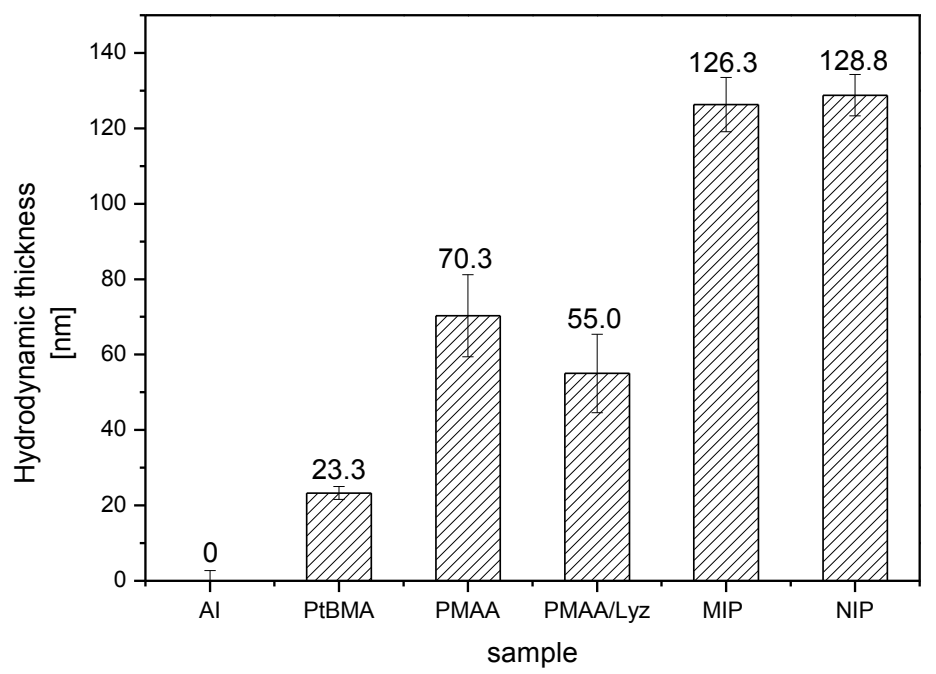

Figure 4.29 Hydrodynamic layer thickness of grafted polymer on the PET pore walls of different membranes in water / ethanol $(1: 1, \mathrm{pH}=7.2)$ : Al corresponds to a membrane after ATRP initiator immobilization; TE PET with grafted PtBMA (after $3 \mathrm{~h}$ ATRP) and the subsequently obtained grafted PMAA had DG values of $1.62 \mu \mathrm{g} / \mathrm{cm}^{2}$ and $0.94 \mu \mathrm{g} / \mathrm{cm}^{2}$, respectively; the same UV-initiated grafting/crosslinking conditions, 10\% crosslinking degree (i.e., MBAA fraction in the monomer mixture) and 20 min UV time, had been used for MIP and NIP synthesis using PET-g-PMAA with DG $=0.94 \mu \mathrm{g} / \mathrm{cm}^{2}$.

After ATRP initiator immobilization, the morphology of the TE PET membrane had not been changed compared with the base membrane. ${ }^{[14]}$ The same permeability corresponds to an added hydrodynamic layer thickness on the surface of zero. After PtBMA grafting with DG = $1.62 \mu \mathrm{g} / \mathrm{cm}^{2}$, the hydrodynamic layer thickness was $23 \mathrm{~nm}$. This is very close to the dry layer thickness (polymer in fully collapsed state) which was estimated from gas flow / liquid displacement data to be about $21 \mathrm{~nm}$. It indicates that the PtBMA polymer chains were also collapsed in the ethanol / water mixture. This is attributed to the hydrophobic property of the polymers tert.-butyl side groups in a very high density, which has been proved by the contact angle measurements (cf. Figure 4.19). 
After hydrolysis, the hydrodynamic layer thickness was increased significantly to $70 \mathrm{~nm}$. The dry layer thickness of this PMAA (DG $=0.94 \mu \mathrm{g} / \mathrm{cm}^{2}$ ) estimated from gas flow / liquid displacement data was only $9 \mathrm{~nm}$, very well in line with the loss of mass during hydrolysis (cf. Figure 4.18). The carboxylic acid groups of the PMAA are fully ionized at $\mathrm{pH}=7.2$ in aqueous solution, this results in a strong swelling of the PMAA layer. The relatively large ratio between swollen and dry layer thickness indicates that the grafting density is not as high as expected for the brush regime. ${ }^{[11,142-144]}$ The density of the grafted polymer chain is not high enough, the distance between the polymer chains is big and the polymer chains were not forced to stretch in the vertical direction but collapse in the horizontal direction like a mushroom form.

After protein adsorption, the hydrodynamic thickness of the PMAA layer was reduced from $70 \mathrm{~nm}$ to $55 \mathrm{~nm}$ due to the formation of the PMAA/Lyz complexes. PMAA has multiple binding sites within a single macromolecular chain that can wind around one or several Lyz molecules. At the same time, protein can penetrate deeply into the PMAA layer and can maximize the favorable binding interactions to the scaffold layer. ${ }^{[134]}$ Part of the charge $\mathrm{COO}^{-}$groups was neutralized after protein adsorption, the repeling interactions between $\mathrm{COO}^{-}$groups on the chains was reduced. Finally, the structure of PMAA layer became denser after protein adsorption. ${ }^{[145]}$

Based on quantitative data for Lyz binding (cf. Figure 4.14) and the hydrodynamic size of Lyz (cf. Table 4.4), it can be estimated that about 10 monolayers of Lyz can be bound to a scaffold layer with that particular DG. This is also in approximate agreement with the estimated hydrodynamic thickness of the grafted PMAA/Lyz layer.

After grafting/crosslinking copolymerization of the PAAm-based hydrogel layer, the MIP had a hydrodynamic layer thickness of $128 \mathrm{~nm}$ (compared to $55 \mathrm{~nm}$ for PET-g-PMAA/Lyz). This is similar to that of the NIP with $131 \mathrm{~nm}$ (compared to $70 \mathrm{~nm}$ for PET-g-PMAA). Obviously the increase of thickness was slightly larger for MIP than for NIP. Possible reasons are a higher degree of grafting due to the higher effective photo-initiator $(\mathrm{C}-\mathrm{Br})$ density in the more compact PMAA/Lyz layer or a partial reswelling upon template removal after the photo grafting step. With view on the concept (cf. Figure 1.1) excessive hydrogel layer growth and crosslinking should be avoided. Therefore, attempts to optimize the two-step imprinting were undertaken. 


\subsubsection{Optimization of the UV-grafting/crosslinking time}

The imprinting effect after variation of the UV-grafting/crosslinking time from 10 min to 25 min was investigated. The binding capacity and selectivity were determined from measurement of protein sorption from $0.2 \mathrm{~g} / \mathrm{L}$ Lyz (or $\mathrm{CyC}$ ) single protein solutions (Figure 4.30).

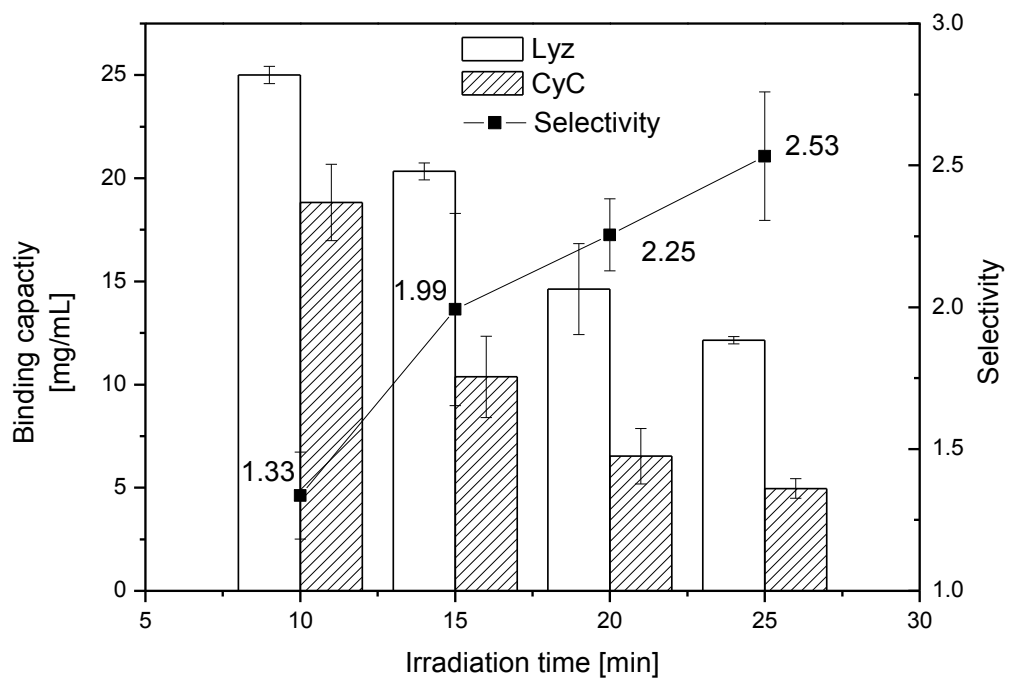

Figure 4.30 Influence of UV-grafting/crosslinking time on the properties of Lys-imprinted TE PET membranes: the Lyz and CyC binding capacities (open and solid columns) and resulting Lyz vs. CyC selectivity (squares) of the different MIP membranes with the same length of scaffold chains (ATRP: DG PMAA $_{1} 1.29 \mu \mathrm{g} / \mathrm{cm}^{2} ; U V: 0.3 \mathrm{~mol} / \mathrm{L}$ functional monomer; AAm:MBAA = 9:1, i.e. degree of crosslinking 10\%; UV-intensity about $24 \mathrm{~mW} / \mathrm{cm}^{2}$ ).

Increasing the UV-grafting/crosslinking time from $10 \mathrm{~min}$ to $25 \mathrm{~min}$ led to an increase of the imprinting effect, the selectivity of Lyz vs. CyC was improved from 1.3 to 2.5. More PAAmbased hydrogel was reactively filled around the scaffold/protein complex, to obtain a higher yield of imprinted cavities after the template protein elution. However too large hydrogel layer thickness led to a poor mass transport of protein in the imprinted polymer layer (or probably also to less efficient template removal), the protein binding capacities were reduced with higher UV-grafting/crosslinking time. In addition, the longer UV irradiation time produced more heat in the reaction mixture resulting in an increase of the temperature, which can affect the native conformation of the protein and reduce the control on the grafting/crosslinking reaction. ${ }^{[146]}$ This will play a negative role in preparing reproducibly high 
quality imprinted structures. Considering the control of UV-grafting/crosslinking, protein binding capacity and MIP selectivity, the 20 min irradiation time was chosen for the further investigations.

\subsubsection{Optimization of the scaffold length}

To improve the recognition property of the imprinted membrane adsorbers, the length of scaffold was optimized by adjusting different degree of grafting through SI-ATRP time (cf. Figure 4.18). The membranes with different scaffold chain lengths had, after adsorbing the template Lyz, then been further UV-functionalized with PAAm hydrogel under identical conditions. A trade-off between binding capacity and selectivity was observed; the highest selectivity of Lyz to CyC was obtained from the shortest scaffold chain length (Figure 4.31).

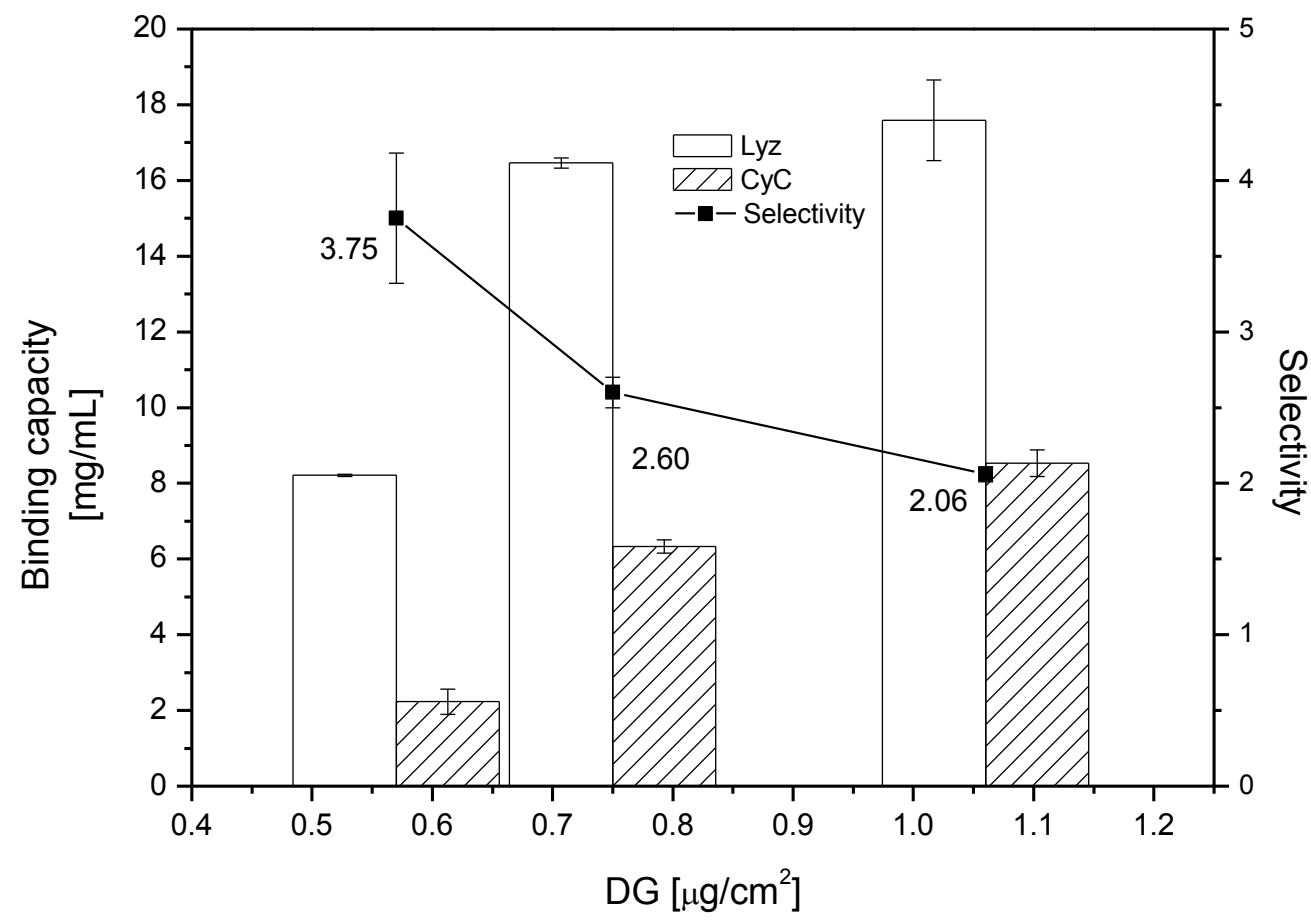

Figure 4.31 Effect of scaffold chain length (DG) on the Lyz and CyC binding capacities (open and solid columns) and resulting Lyz vs. CyC selectivity (squares) of the different MIP membranes. The UV-initiated grafting/crosslinking was carried out in $0.3 \mathrm{~mol} / \mathrm{L}$ functional monomers (AAM:MBA = 9:1, i.e. degree of cross linking 10\%) for 20 min under the UVintensity about $20 \mathrm{~mW} / \mathrm{cm}^{2}$. 
It can be seen that the reduction of the scaffold chain length was an effective method to improve the recognition selectivity of the MIP layer, although the binding capacity was reduced. Note that the grafted layers with $D G=0.94 \mu \mathrm{g} / \mathrm{cm}^{2}$ had a hydrodynamic layer thickness of about $70 \mathrm{~nm}$ without and $50 \mathrm{~nm}$ with bound protein (cf. Figure 4.29). Too much adsorbed protein due to the long grafted scaffold chains (much more than capacity corresponding to a monolayer) led to more interactions between protein molecules and non-specific interactions between protein and scaffold. These cause a reduction of the fraction of solvated surface of the bound protein and of the fraction of specific interactions; $^{[147]}$ therefore, the imprinted structure with long scaffold chains had deteriorated recognition property after imprinting.

In this work, the motivation for using SI-ATRP was to make use of the precise optimization of scaffold chain length to improve the imprinting properties. Through the two-step grafting, the complex imprinting process was divided into two parts, and in each part the optimization can be performed individually which could be more rational and effective in comparison with the conventional single step imprinting.

\subsubsection{Optimization of the crosslinking degree}

After the scaffold variation the improvement of the MIP property was investigated via variation of crosslinking degree. The crosslinking degree of the hydrogel was optimized with the variation of the MBAA content from $10 \%$ to $15 \%$ relative to total monomer (AAm + MBAA); results are shown in Figure 4.32. 


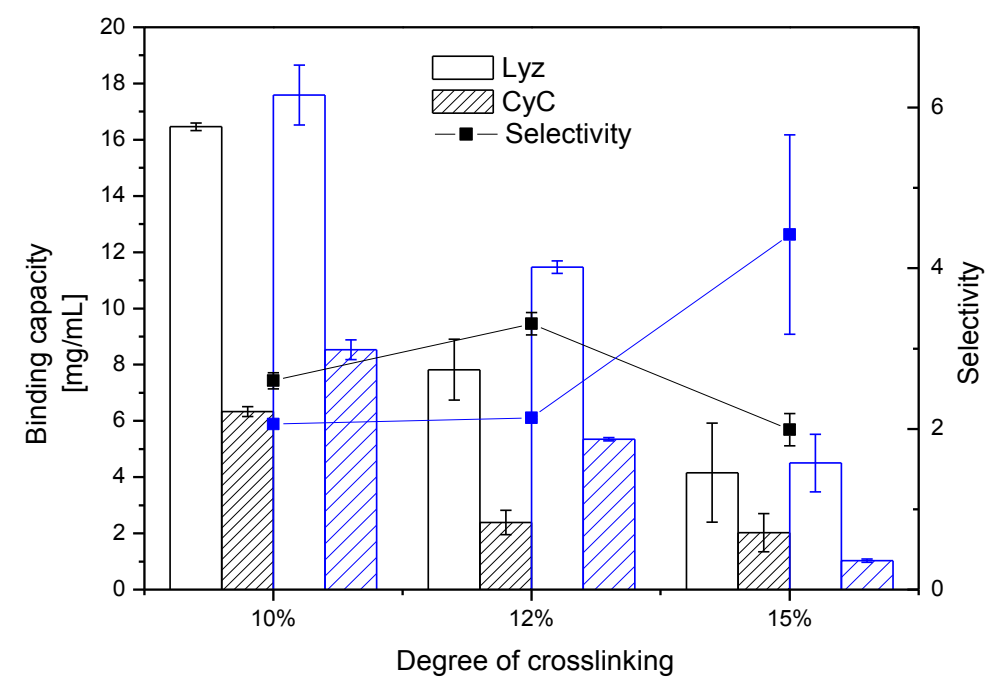

Figure 4.32 Effect of crosslinking degree on protein binding capacity and Lyz to CyC selectivity of MIP membranes with different scaffold DG. Open and solid columns represent the binding capacities of Lyz and CyC, respectively, squares the Lyz to CyC selectivity. Black represents the series of MIP membranes with scaffold DG $=0.75 \mu \mathrm{g} / \mathrm{cm}^{2}$, blue represents the series of MIP membranes with scaffold DG $=1.06 \mathrm{~g} / \mathrm{cm}^{2}$.

For the scaffold with DG $=0.75 \mu \mathrm{g} / \mathrm{cm}^{2}$ a maximum Lyz to CyC selectivity of 3.3 was achieved with cross-linking degree of $12 \%$, and for the scaffold with $D G=1.06 \mu \mathrm{g} / \mathrm{cm}^{2}$ the maximum, a selectivity of 4.2 , was shifted to a crosslinking degree of $15 \%$. The degree of crosslinking should be high enough in order to reduce the hydrogel swelling and maintain the stability of the imprinted cavities in the PAAm-based hydrogel. ${ }^{[148]}$ With the increase of cross-linking degree, the polymer fraction in the swollen hydrogel was increased which led to increased hindrance for protein transport in the hydrogel and thus reduced the protein binding capacity. However, decreasing the cross-linking leads to formation of soft imprinted cavities, which results in a less efficient "induced fit" effect with reduction of the recognition property. ${ }^{[149-150]}$ The other scaffold layer $\left(D G=1.06 \mu \mathrm{g} / \mathrm{cm}^{2}\right)$ demonstrated an increasing tendency when the crosslinking degree increased from $10 \%$ to $15 \%$. In comparison with DG = $0.75 \mu \mathrm{g} / \mathrm{cm}^{2}$ series, the initiator density for UV-initiation (bromide chain end groups within the polymer layer; cf. Scheme $\mathbf{1 . 1}$ and 1.2) was lower in the scaffold/protein complex layer, this can probably partially counteract the effect of polymer density increase which results from the increase of the crosslinker monomer fraction and yield a more open PAAm-based hydrogel. 


\subsubsection{Comparison of MIP, NIP and scaffold membranes}

Considering the achieved Lyz binding capacity and Lyz vs. CyC selectivity as well as the experimental repeatability (the highest crosslinker fraction lead to larger variation of experimental data; cf. Figure 4.32), the MIP adsorbers based on scaffold DG $=0.75 \mu \mathrm{g} / \mathrm{cm}^{2}$ and UV-grafting/crosslinking copolymerization at $12 \%$ crosslinking degree were used for the further detailed characterization. The specific recognition was investigated by comparing the protein binding capacity and selectivity with control materials, i.e. the precursor scaffold and the respective NIP (Figure 4.33).

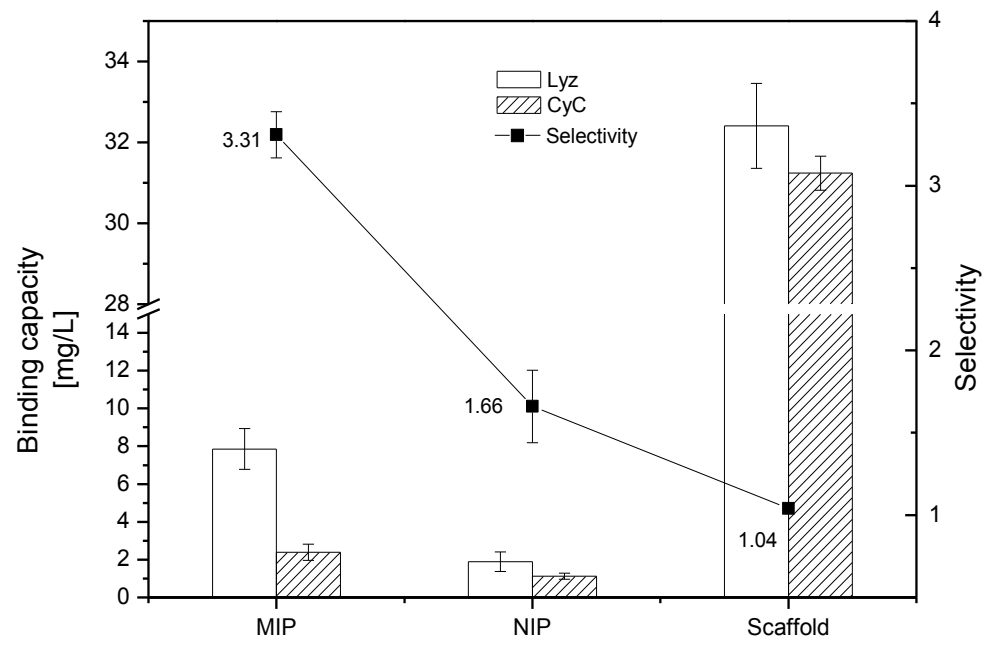

Figure 4.33 Lyz (open columns) and CyC (solid columns) binding capacities and Lyz to CyC selectivities (squares) for MIP, NIP and scaffold membranes (scaffold DG $=0.75 \mu \mathrm{g} / \mathrm{cm}^{2} ; 12 \%$ crosslinking degree).

The scaffold membrane had a capacity of about $32 \mathrm{mg} / \mathrm{mL}$ for Lyz and showed essentially no selectivity for Lyz vs. CyC ( 1). After functionalization with the PAAm-based hydrogel, a large fraction of the scaffold binding sites was blocked, so that the binding capacities of MIP and NIP were reduced significantly. However, the imprinting effect was proven by comparing MIP and NIP membranes. The MIP structure showed a Lyz binding capacity of $7.8 \mathrm{mg} / \mathrm{mL}$ and also a higher Lyz to CyC selectivity of 3.3, compared to the values of NIP which had a Lyz binding capacity of $1.9 \mathrm{mg} / \mathrm{mL}$ and Lyz to CyC selectivity of 1.7. Due to the presence of template protein during the cross-linking process, the functional groups from scaffold PMAA 
and monomer AAm were arranged on the template protein surface in order to maximize the favorable interactions with the protein. After UV-initiated polymerization and template elution the cavities with complementary size, shape and functional groups arrangement were left in the cross-linked hydrogel layer. In the protein rebinding experiment, these imprinted cavities lead to the higher protein capacity and higher selectivity of MIP in comparison with NIP membranes (at about the same grafted polymer layer thickness; measured for similar materials, cf. Figure 4.29).

\subsubsection{Separation of protein mixtures}

The Lyz selectivity of the MIP membrane was further investigated in a $0.2 \mathrm{~g} / \mathrm{L}$ protein mixture solution of Lyz and CyC (1:1), which was considered difficult to separate by using the conventional technologies due to their similar size and isoelectric point. The adsorbed amounts of Lyz and CyC were determined by the enzyme assay with Micrococcus lysodeikticus and direct UV-Vis spectroscopy, respectively. The results are shown below (Figure 4.34).

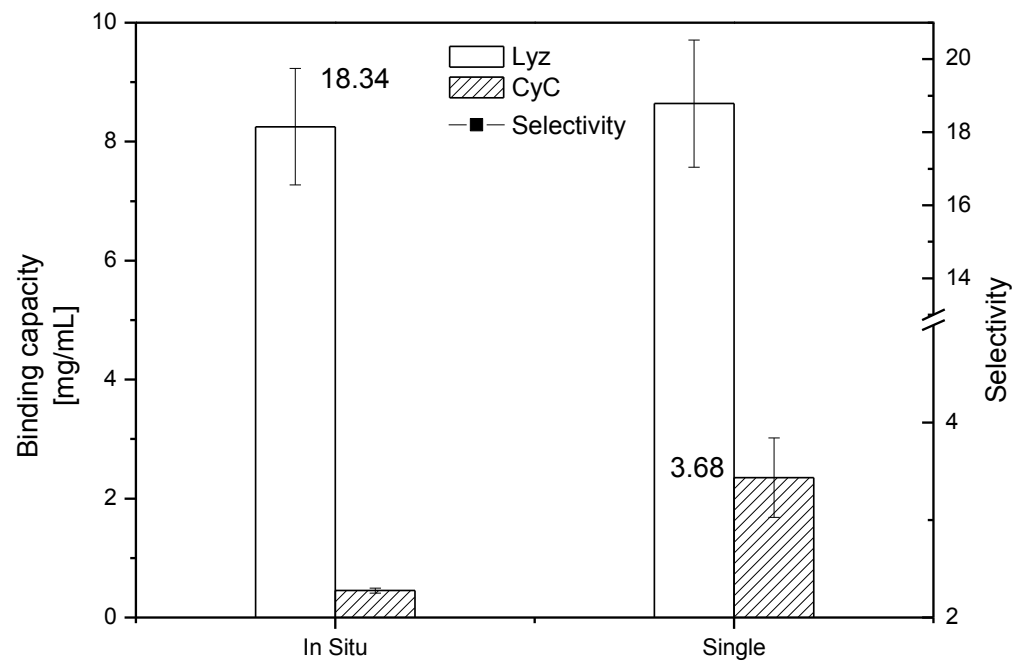

Figure 4.34 Lyz (open columns) and CyC (solid columns) binding capacities and Lyz to CyC selectivity (squares) of MIP membranes (scaffold DG $=0.75 \mu \mathrm{g} / \mathrm{cm}^{2} ; 12 \%$ crosslinking degree) obtained in single protein experiments $(0.2 \mathrm{~g} / \mathrm{L})$ and from in situ separation of a 1:1 protein mixture $(0.1+0.1 \mathrm{~g} / \mathrm{L})$ at $\mathrm{pH}=7.0$. 
By the experiment with the protein mixture, the MIP effect was demonstrated much more clearly than in two single protein experiments. The MIP membrane had a similar Lyz binding capacity during in situ mixture and single protein binding measurements, but the Lyz to CyC selectivity during in situ process increased to 18.3 (from 3.7 from single protein experiments). In the in situ, competitive adsorption process, the MIP membrane discriminated the proteins not simply on the basis of a size-exclusion mechanism or by simple charge complementarity, but by the synergistic effect of size, shape and fitting multiple weak interactions (electrostatic interaction, hydrogen bonding and hydrophobic interaction) with the template protein provided by imprinted sites. The results support strongly that imprinted sites have been created which have a much higher affinity and selectivity for Lyz than for CyC. When using single protein solutions at relatively high concentration (here $0.2 \mathrm{~g} / \mathrm{L}$; cf. section 3.3.3), high and lower affinity sites (including simple cation-exchange groups) will bind protein. In the competition experiment, the high affinity sites for Lys will only be occupied by Lys, thus reducing the bound amount of $\mathrm{CyC}$.

\subsubsection{Protein binding isotherms of MIP and NIP membranes}

In order to gain more insights into the binding properties of the MIP adsorber and to quantify MIP specificity and affinity, protein binding isotherms were measured and fitted to the Langmuir model; the results are shown in Figure 4.35.

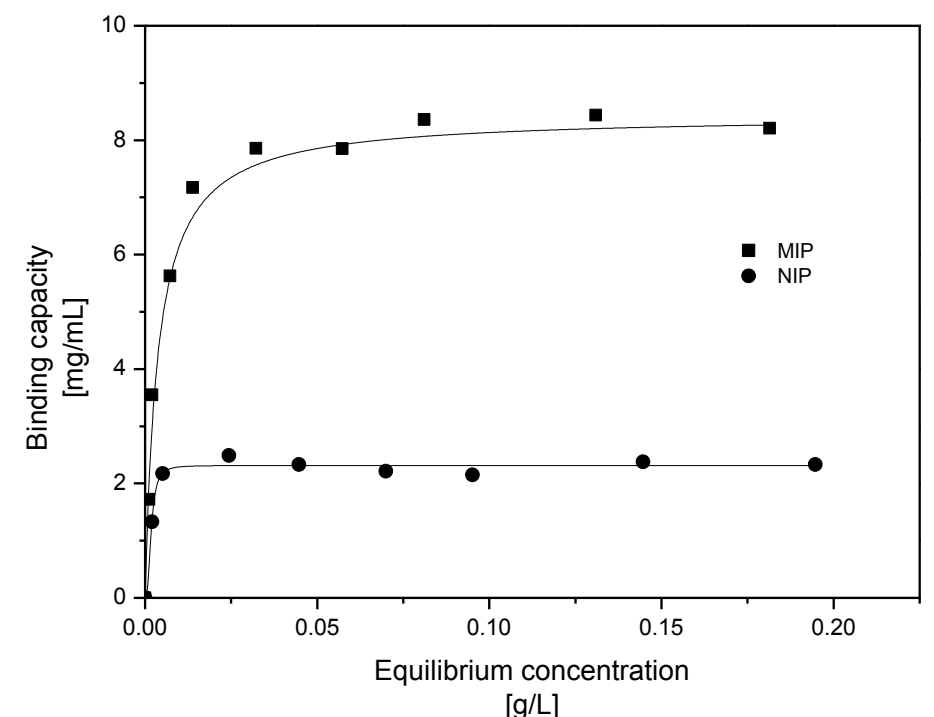

[g/L] 
Figure 4.35 Lyz binding isotherms of MIP (solid squares) and NIP (solid circles) membranes as well as curves obtained by fitting the data to the Langmuir model.

The MIP membrane had a much higher binding capacity than the NIP membrane for the template protein. Maximal binding capacities of Lyz reached about $8.3 \mathrm{mg} / \mathrm{mL}$ for MIP and about $2.3 \mathrm{mg} / \mathrm{mL}$ for NIP membranes. The much higher values for MIP vs. NIP at same polymer composition may be related to the high protein loading of the scaffold. The protein seems to create a "pore" structure in the MIP layer so that more sites are accessible for binding.

The protein binding affinity was characterized through calculating the binding constant $\left(\mathrm{K}_{\mathrm{L}}\right)$ in the frame of the Langmuir model. $\mathrm{K}_{\mathrm{L}}$ for Lyz binding to the MIP membrane was $599 \mathrm{~mL} / \mathrm{mg}$ (corresponding to $8.4 * 10^{7} \mathrm{~L} / \mathrm{mol}$ ). The binding of Lyz on NIP membrane was significantly less favorable with a $\mathrm{K}_{\mathrm{L}}$ of $216 \mathrm{~mL} / \mathrm{mg}\left(3.0 * 10^{7} \mathrm{~L} / \mathrm{mol}\right)$. The latter value is somewhat larger than what had been obtained for the protein-selective adsorber based on linear grafted chains with special synthetic recognition groups (Lyz: $\mathrm{K}_{\mathrm{L}} 1.3 * 10^{7} \mathrm{~L} / \mathrm{mol}$; Lyz vs. CyC selectivity $\sim 15) .{ }^{[12]}$ In the literature ${ }^{[151]}$ a large set of Lyz antibodies had been evaluated vs. a range of antigens (Lyz from different species) and the affinity constants were found to be in a range from 5.3 to $0.8^{*} 10^{7} \mathrm{~L} / \mathrm{mol}$. The binding constant of MIP reached in this work is in the range of the strengths of nature's antibody-antigen interactions.

Overall, the MIP membrane adsorber obtained here using simple commercial monomers and robust grafting technologies had higher binding constant, higher Lys vs. CyC selectivity and much higher capacity in comparison with the material from the work of He et al. who had used the same base membrane but a more complex special monomer, i.e. an aromatic bisphosphonate derivative, for preparation of linear functional chains in a single-step grafting procedure. ${ }^{[12]}$

\subsubsection{Effect of ionic strength onto protein binding}

In this study a relatively simple scaffold, PMAA, providing a relatively high density of cationexchange groups has been used. As seen and discussed under section 4.6.3, this could lead to a relatively large non-specific background binding under low salt conditions. Therefore, the effect of ionic strength on the Lyz binding was also investigated (Figure 4.36). 


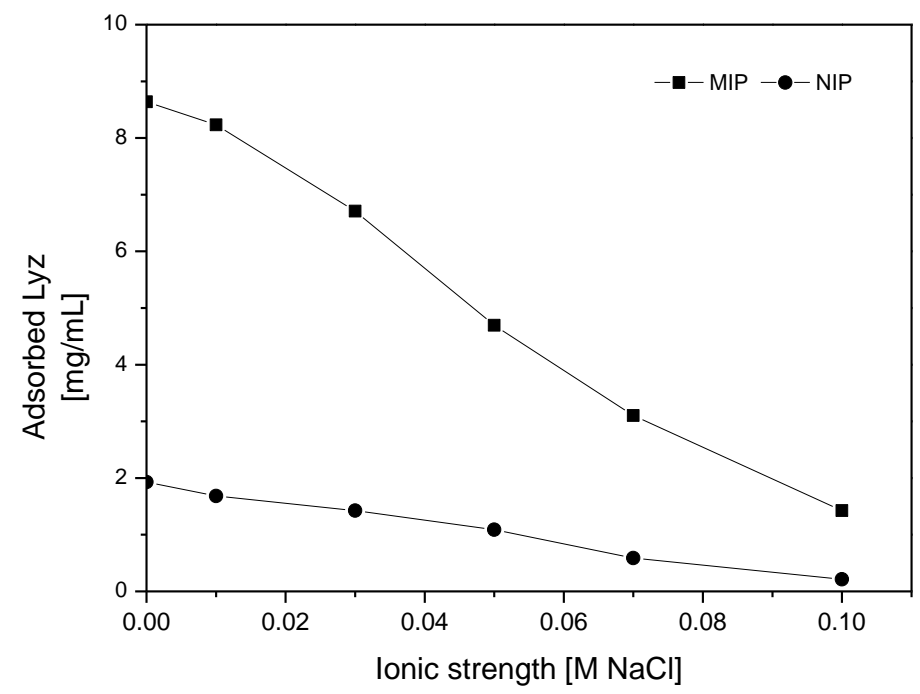

Figure 4.36 Effect of ionic strength on Lyz binding to MIP (squares) and NIP (circles) membranes (protein concentration $0.2 \mathrm{~g} / \mathrm{L}$, phosphate buffer $\mathrm{pH}=7$ ).

Increasing ionic strength weakened the binding for both materials. Lyz capacity of the MIP membrane reduced from 8.8 to $1.4 \mathrm{mg} / \mathrm{mL}$ with the increasing $\mathrm{NaCl}$ concentration from 0 to $0.1 \mathrm{~g} / \mathrm{L}$. This also proved that the affinity between protein and MIP structure was not homogenous, a smaller fraction of the binding sites hat very high affinity to Lyz even at a relatively high salt concentration. The electrostatistic interaction played an important role in the specific Lyz binding to the MIP membrane; the additive salt ions screened the interactions between proteins and non-specific binding sites.

In the Lyz binding experiment with the $\mathrm{NaCl}$ concentration from 0 to $0.05 \mathrm{~g} / \mathrm{L}$, the Lyz binding capacity of NIP membrane showed only a slight reduction in comparison with the result of MIP membrane. This is due to the ratio change of cooperative effects such as ion-exchange effect, hydrophobic interaction and hydrogen binding. By the Lyz binding of NIP membrane, the ion-exchange effect may not dominate the interaction between protein and NIP structure as that by the Lyz binding on MIP membranes, other effects play more important role, and the Lyz binding on NIP is not as sensitive to $\mathrm{NaCl}$ as MIP in small concentration area.

The reduce of the binding capacity with the increasing ionic strength in low salt concentration range indicates that the heterogeneity of the special binding sites of the MIP structure to Lyz was high, this was the limitation of the surface imprinting when only portion of the protein surface was imprinted and the functionality of scaffold was too weak to form 
enough stable protein/scaffold complex. Using the scaffold with multi binding sites and also multi functionalities as an extender of the PMAA scaffold with multi binding sites but only single functionality is considered to overcome the binding heterogeneity. Long et al. presented a similar protein imprinting strategy by using the assistant recognition polymer chains (ARPCs) to imprint pig cyclophilin 18 (pCyP18), and the introduced pyridyl group in the negative charged ARPCs with hydrophobic effect element can enhance the recognition property of the MIP structure. ${ }^{[8]}$

\subsubsection{Introduction of more additive monomers during UV-grafting/crosslinking}

Isoelectric point just presents the net surface charge of protein surface, but the real residue distribution containing positive, negative, hydrophobic, and hydrophilic segments is very complicated and sensitive to environment such as $\mathrm{pH}$ value, salt concentration, and polarity. Polymer with complementary reactive sites to the protein surface is proposed to improve the affinity of imprinted structure. SI-ATRP of the monomers with multi functionalities such as positive and negative charges is difficult and lacking in precise characterization. However copolymerization with multi functional monomers by UV-grafting/crosslinking is easier to practice. The advantage of two-step grafting was demonstrated again, the difficult optimization can be divided to more simple elements and carried out in separate step, ATRP and UV-grafting/crosslinking, respectively. Hydrophilic monomer HEMA and positively charged monomers DEMAEMA, DMPAA, and APMA.HCl were combined to the PAAm backbone during the UV-grafting/crosslinking (Figure 4.37) and the imprinting results are shown in Figure 4.38.<smiles>C=C(C)C(=O)OCCO</smiles>

HEMA

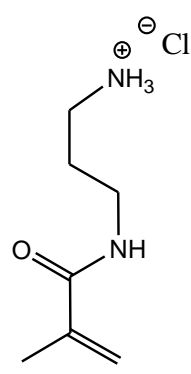

APMA• HCI

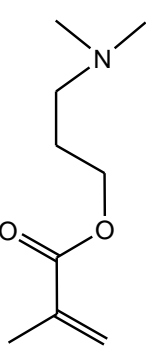

DMAEMA

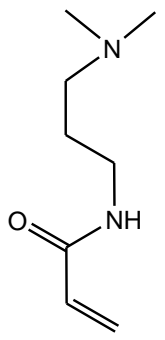

DMPAA

Figure 4.37 Chemical structures of additive functional monomers during the UVpolymerization. 


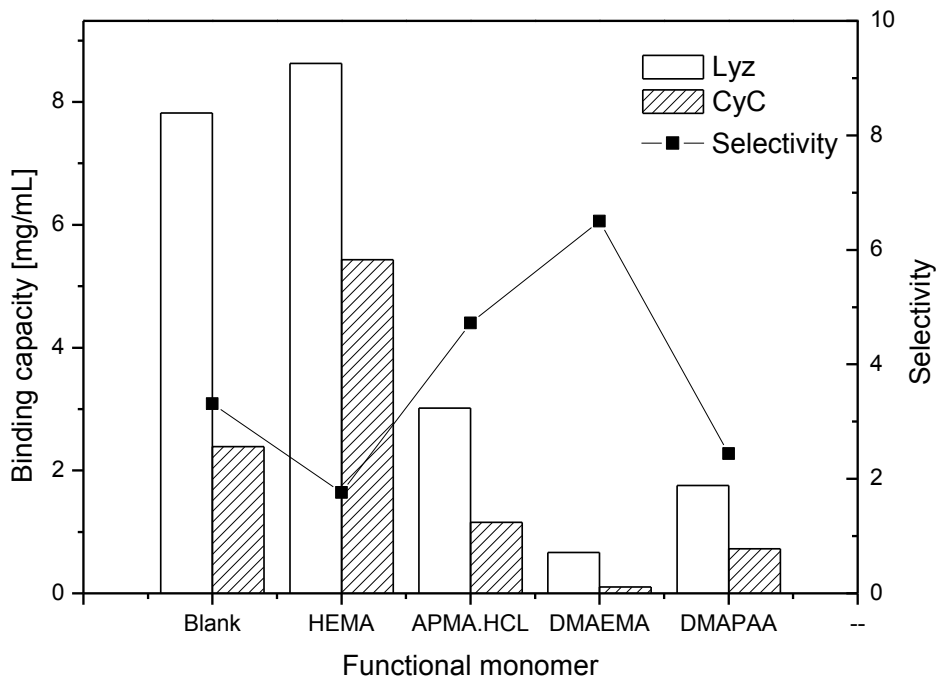

Figure 4.38 Lyz (open columns), CyC (solid columns) binding capacities and selectivity of Lyz to CyC (squares) of MIP membranes (scaffold DG PMAA $\approx 0.80 \mu \mathrm{g} / \mathrm{cm}^{2}$ ), UVgrafting/crosslinking: $0.3 \mathrm{~mol} / \mathrm{L}$ monomer concentration with $\mathrm{AAm}: \mathrm{MBAA}$ :additive = 7.8:1.2:1, irradiation under $20 \mathrm{~mW} / \mathrm{cm}^{2}$ for $20 \mathrm{~min}$.

The blank sample without added functional monomer during the UV-grafting/crosslinking has been shown in Figure 4.33. Lyz imprinted membrane with addition of HEMA during UVgrafting/crosslinking had both higher capacities of Lyz and CyC than those of the blank sample but the selectivity of Lyz to CyC was reduced significantly from 3.31 to 1.76. Increased capacities of Lyz and CyC resulted partially from the slightly longer PMAA chain $\left(D G_{\text {blank }}=0.75 \mu \mathrm{g} / \mathrm{cm}^{2}<\mathrm{DG}_{\text {Additive }} \approx 0.80 \mu \mathrm{g} / \mathrm{cm}^{2}\right)$. The UV-reaction solution including HEMA with a original $\mathrm{pH}$ value lower than 4 , more $\mathrm{NaOH}$ was added to calibrate the $\mathrm{pH}$ to 7 than that of the solution without HEMA, this led to a more swelling structure of PMAA and provide more space for protein adsorption and less dense PAAm based hydrogel filling, therefore the imprinting effect was reduced.

Addition of APMA.HCl increased the selectivity of Lyz to CyC from 3.31 to 4.72, notwithstanding the capacities of Lyz and CyC were reduced. Additive APMA.HCl reduced the $\mathrm{pH}$ value of UV reaction solution. $\mathrm{pH}$ was set to 7.0 via addition of $\mathrm{NaOH}$, thereafter more salt was formed in the solution. This led a lower protein binding capacity. Salt shrank the PMAA and shielded the interaction between protein and carboxyl group as counter ion. 
The maximum selectivity of Lyz to CyC was obtained by addition of DMAEMA in the UVreaction solution with value of 6.50 , but the protein binding capacity was reduced to less than $1 \mathrm{mg} / \mathrm{mL}$ which resulted from the addition of $\mathrm{HCl}$ to calibrate the $\mathrm{pH}$ value of the UVreaction solution to $7.0 . \mathrm{HCl}$ led to break the weak electrostatic interactions between protein and PMAA chain via protonation of the carboxyl group. So the capacity of protein was reduced very significantly, but the rest stronger electrostatic interaction between protein and scaffold resulted in a high recognition property according to the typical molecular imprinting theory. Addition of DMPAA, also with $\mathrm{pH}$ calibration via addition of $\mathrm{HCl}$ in UVreaction solution, but both the protein binding capacity and selectivity were reduced.

The calibration of the $\mathrm{pH}$ via addition of acid or base reduced the protein binding capacity. However, the addition of negatively/positively charged functionalities increased the selectivity of imprinted structure. This strategy was also applied by Shea and his coworkers to imprint bee toxin melttin by using the polymer with multi functionalities via copolymerization of acrylic acid (negative- charged), N-(3-aminopropyl) methacrylamide (positive-charged), and N-tert-butyl acrylamide (hydrophobic). ${ }^{[152]}$ Searon et al. presented a work to improve the property of maltose binding protein via optimization of the monomer composition from AAm, MBAAA, MAA and N-[3-(dimethylamino) propyl] methacrylamide. The simulation of maltose binding protein surface residues provide important information to design the monomer composition. ${ }^{[150]}$ 


\subsection{Immunoglobin G imprinting on PET membrane}

The two-step grafting has been successfully applied for the imprinting of Lyz, the property of the Lyz imprinted structure was optimized by the first scaffold grafting and the subsequent UV-grafting/crosslinking independently and respectively (cf. 4.6). The size of Lyz with MW about $14.4 \mathrm{kDa}$ is smaller in comparison with BSA (66.4 kDa), protein A (56 kDa), IgG (150 $\mathrm{kDa}$ ). For protein imprinting, this strategy should further tested by the imprinting of the proteins with bigger size and more complicate conformation, therefore IgG was chosen as template to investigate the practicability of this two-step strategy. IgG is the main antibody to control the infection of body tissues which has been found in blood and extracellular fluid. IgG has Y-shape composed of 4 protein chains, two identical heavy chains (chain MW about $50 \mathrm{kDa}$ ) and two identical light chains (chain MW about $25 \mathrm{kDa}$ ).

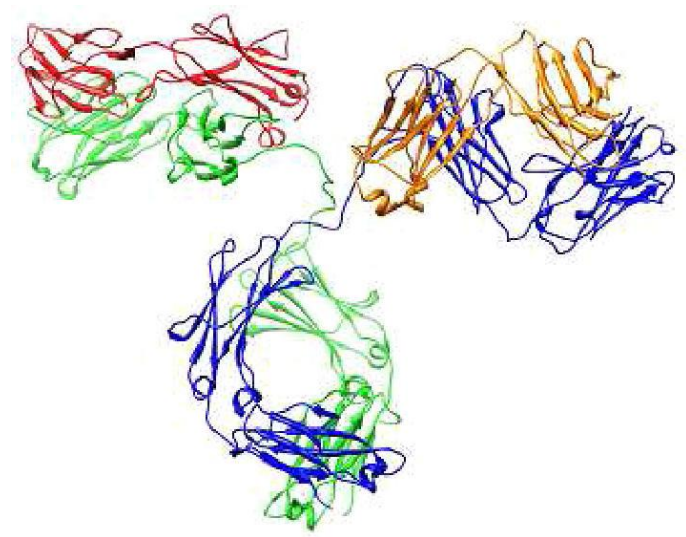

Figure 4.39 Structure of IgG with heavy chains in blue and green and light chains in orange and red. ${ }^{[153]}$

HSA was used for the competitive adsorption to characterize the property of the IgG imprinted structure. Separation the mixture of IgG and HSA which is a typical biological backgroud attracted considerable attention for its great potential in blood protein manufacture. ${ }^{[154]}$

Table 4.5 Information on IgG and HSA.

\begin{tabular}{c|c|c}
\hline & IgG & HSA \\
\hline $\mathrm{MW}[\mathrm{kDa}]$ & $\sim 150$ & $\sim 68$ \\
\hline $\mathrm{pl}$ & $5.8-7.3$ & 4.8 \\
\hline Size $\left[\mathrm{nm}^{3}\right]^{[155]}$ & $21.9 * 15.5 * 1.5$ & $8 * 6.9 * 3$ \\
\hline
\end{tabular}




\subsubsection{Optimization of the UV-grafting/crosslinking time}

According to the result of scaffold optimization in section 4.5 .3 , a short grafted PMAA chain was tested at first with $D G=0.58 \mu \mathrm{g} / \mathrm{cm}^{2}$. IgG has a molecular dimension $21.9 * 15.5 * 1.5 \mathrm{~nm}$ with a hydrodynamic diameter of approximately $12 \mathrm{~nm}$ at $\mathrm{pH}=7$ in aqueous solution. ${ }^{[155]}$ The big size of IgG leads to theoretically a poor transfer in the imprinted polymer matrix, so the low degree of crosslinking $10 \%$ and short UV-irradiation time with range from 10 to $15 \mathrm{~min}$ were applied to start the optimization. The result is shown in Figure 4.41.

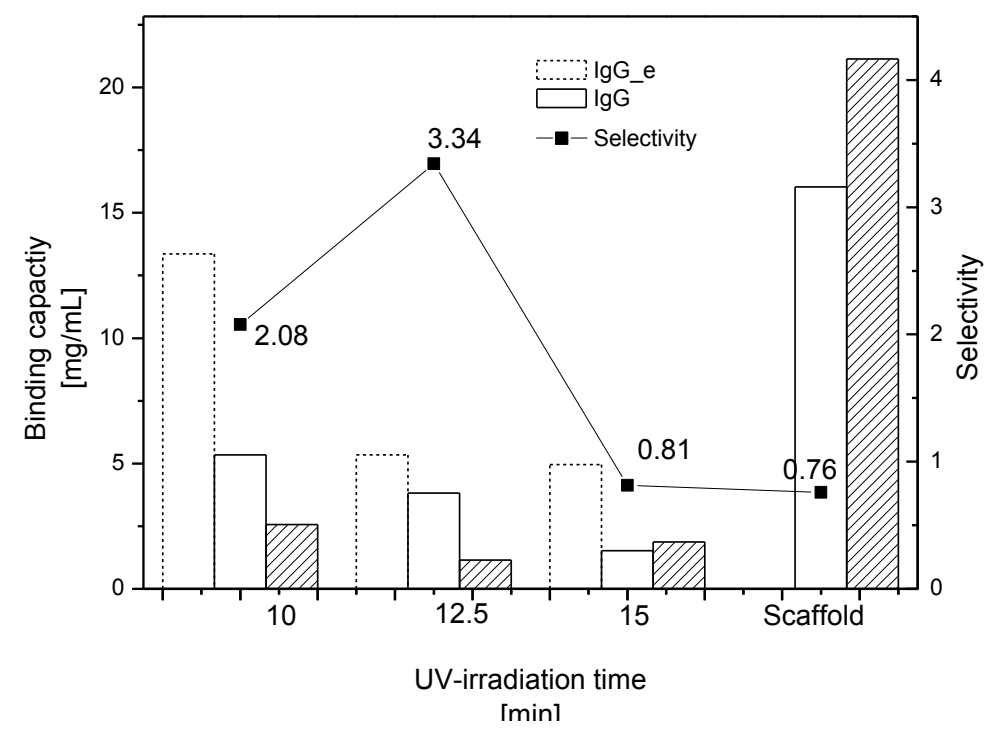

Figure 4.40 Eluted IgG after crosslinking (dash open columns), rebinding IgG (open columns), HSA (solid columns) binding capacities, and IgG to HSA selectivity (squares) of MIP membranes (scaffold $D_{\text {PMAA }} \approx 0.58 \mu \mathrm{g} / \mathrm{cm}^{2}$ ), UV-grafting/crosslinking: $0.3 \mathrm{~mol} / \mathrm{L}$ monomer concentration with $\mathrm{AAm}: \mathrm{MBAA}=9: 1$, i.e. crosslinking degree $=10 \%$, irradiation under 20 $\mathrm{mW} / \mathrm{cm}^{2}$ for 10 to $15 \mathrm{~min}$.

The scaffold of PMMA hat a higher capacity of HSA than that of IgG, this is different to the adsorption of Lyz and CyC (cf. Figure 4.33). The much bigger size of IgG is supposed to be the main reason. The grafted polymer structure self has a size exclusion effect on the protein adsorption, ${ }^{[156]}$ IgG with big size can not diffuse into the deep PMAA polymer layer. After imprinting, both IgG and HSA binding capacities were much less than those of Lyz and CyC (cf. Figure 4.31). A maximum IgG to HSA selectivity of 3.34 was achieved by $10 \%$ crosslinking degree and UV-irradiation time $12.5 \mathrm{~min}$ with the scaffold of DG $=0.58 \mu \mathrm{g} / \mathrm{cm}^{2}$. With increased UV-irradiation time, more PAAm based hydrogel was filled around the complex of 
IgG/PMAA, this was supposed to increase the space imprinting effect with high recognition property. However, too much hydrogel led to a poor mass transport of protein in the imprinted polymer layer (or probably also to less efficient template removal), especially for IgG with big size and $Y$ shape. IgG binding capacity were reduced with longer UV-irradiation time. The poor rebinding phenomenon was demonstrated by the comparison of eluted IgG and the rebinding capacity of IgG. This was also the main reason what lead to a poor recognition property and lower rebinding capacity resulted from the long irradiation time. From 12.5 to 15 min irradiation, the IgG capacity of imprinted structure reduced more sharply with 3.8 to $1.5 \mathrm{mg} / \mathrm{mL}$ than that of from $10 \mathrm{~min}$ to $12.5 \mathrm{~min}$ with 5.3 to $3.8 \mathrm{mg} / \mathrm{mL}$. Imprinted structure even demonstrated a higher capacity to HSA than IgG after 15 min irradiation.

By 12.5 min UV-irradiation, the rebinding capacity of IgG has the smallest difference to the eluted capacity directly after crosslinking, it displayed a potential to improve the selectivity via increase of the crosslinking degree.

\subsubsection{Improvement of crosslinking degree}

In consideration of the poor imprinting property after $15 \mathrm{~min}$ irradiation in section 4.7.1, the crosslinking degree was increased slightly from $10 \%$ to $12 \%$ at first with UV-irradiation time $12.5 \mathrm{~min}$.

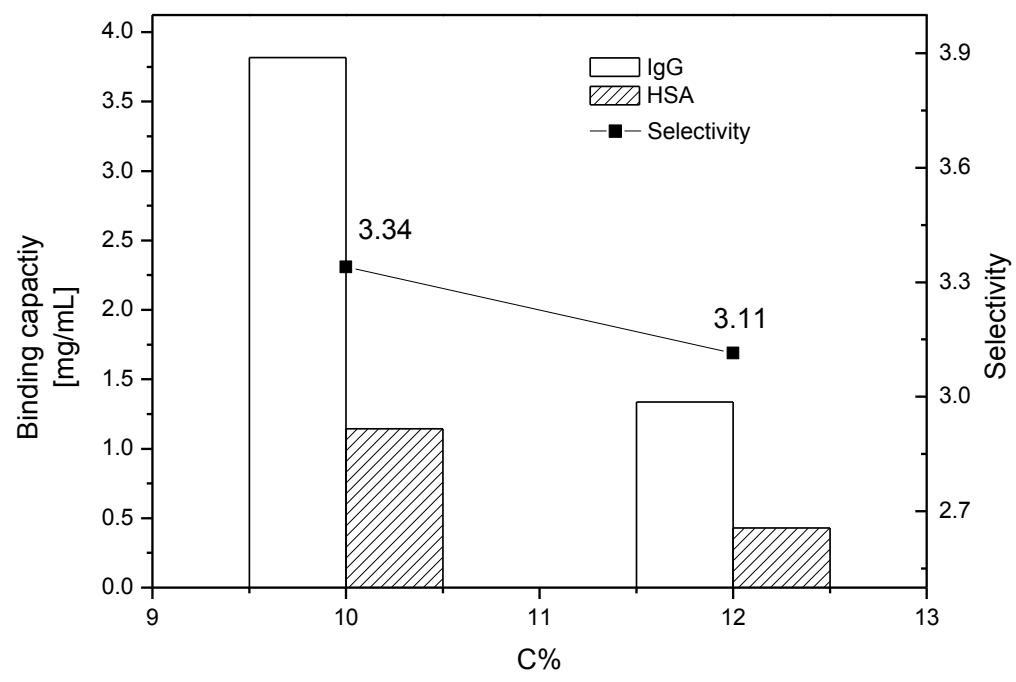


Figure 4.41 IgG (open columns), and HSA (solid columns) binding capacities and IgG to HSA selectivity (squares) of MIP membranes (scaffold DGPMAA $\approx 0.55 \mu \mathrm{g} / \mathrm{cm}^{2}$ ), UVgrafting/crosslinking: $0.3 \mathrm{~mol} / \mathrm{L}$ monomer concentration of AAm and MBAA with crosslinking degree $=10 \%$ and $12 \%$, irradiation under $20 \mathrm{~mW} / \mathrm{cm}^{2}$ for $12.5 \mathrm{~min}$.

Both capacity and selectivity were reduced by improving the crosslinking degree from $10 \%$ to $12 \%$ with $0.58 \mu \mathrm{g} / \mathrm{cm}^{2}$ PMAA scaffold. The IgG seems to be more sensitive to the density of the polymer matrix than Lyz. The most significant difference between IgG and Lyz is size. The big size template led to much poor mass transfer after crosslinking with higher crosslinking degree. The rebinding capacity of IgG reduced from 3.8 to $1.3 \mathrm{mg} / \mathrm{mL}$. According to the slight reduction of selectivity IgG to HSA, the recognition property has not changed as much as the binding capacity with a relative short PMAA scaffold $\left(0.58 \mu \mathrm{g} / \mathrm{cm}^{2}\right)$. So the increase of crosslinking degree led to a poor accessibility for the IgG to the imprinted cavities in the polymer matrix, but it did not improve enough imprinting effect to overcome reduction of mass transfer. Comparing with the imprinting of Lyz, the selectivity over 3 is enough to separate proteins, since the imprinting effect will be enlarged in practice condition such as in situ separation. Further improvement of crosslinking degree seemed to make no sense, because the protein binding capacity was reduced so much.

\subsubsection{Extension of scaffold length}

As discussed in section 4.7.2, the imprinting effect of the cavities was possible been improved, but the reduced mass transfer of $\lg G$ in the polymer matrix dominated over the crosslinking degree effect, and the selectivity demonstrated no significant difference. From the result of Figure 4.31, increase of scaffold chain length, providing more space for protein adsorption and the subsequent crosslinked hyrogel, seem to be an effective method to improve the protein binding capacity. 


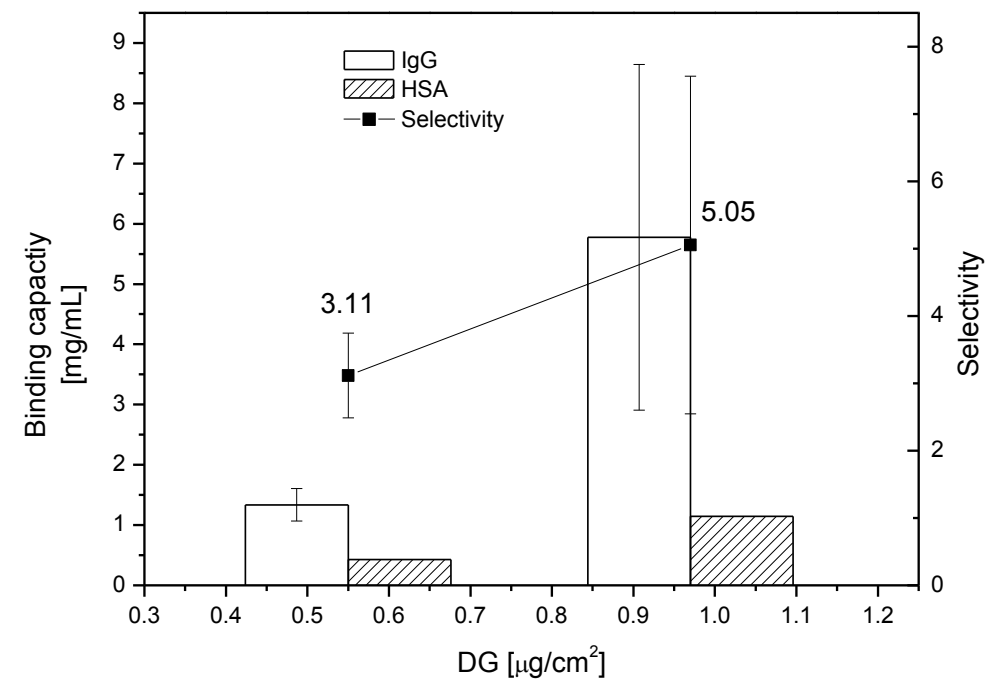

Figure 4.42 IgG (open columns), and HSA (solid columns) binding capacities and IgG to HSA selectivity (squares) of MIP membranes (scaffold DG $_{\mathrm{PMAA}} \approx 0.55$ and $0.97 \mu \mathrm{g} / \mathrm{cm}^{2}$ ), UVgrafting/crosslinking: $0.3 \mathrm{~mol} / \mathrm{L}$ monomer concentration of AAm and MBAA with crosslinking degree $=12 \%$, irradiation under $20 \mathrm{~mW} / \mathrm{cm}^{2}$ for $12.5 \mathrm{~min}$.

At crosslinking degree $=12 \%$, both the protein binding capacity and selectivity haven been increased via extension of PMAA chain from 0.55 to $0.97 \mu \mathrm{g} / \mathrm{cm}^{2}$, the IgG binding capacity increased from 1.3 to $5.8 \mathrm{mg} / \mathrm{mL}$ and selectivity increased from 3.11 to 5.05 . Long scaffold led to a thick protein/scaffold complex layer and provided more free space for the subsequent grafting/crosslinking initiated mainly from the membrane surface (or bottom of the scaffold chain), because the ATRP initiation efficiency is only about $10 \%{ }^{[133]}$ and tertiary bromide (initiator on the membrane surface) is more reactive than secondary bromide (end site of the grafted polymer). After the same initiation, the long scaffold chain provided more free space inner the crosslinked polymer layer than the crosslinking with short scaffold chain, so the mass transfer of protein is better in the polymer layer with long scaffold than that with short scaffold chain after crosslinking under the same condition. For small protein such as Lyz, the better mass transfer increased the binding capacity but reduced the recognition property (cf. Figure 4.31), because the requirement of free space for its transfer inner the polymer matrix was low, however, the situation for large protein IgG is different. Size effect sometimes dominated over all other factors during the adsorption and elution. This effect led also the difference between the scaffold capacities from model IgG/HSA and model $\mathrm{Lyz} / \mathrm{CyC}$ and has been discussed in section 4.7.1. 
With the similar condition, Lyz imprinting has a template binding capacity of Lyz about 12 $\mathrm{mg} / \mathrm{mL}$, which was much higher than that of $5.8 \mathrm{mg} / \mathrm{mL}$ by $\operatorname{lgG}$ imprinting. Similar to the development of imprinting from small organic molecule to protein, the imprinting of big size protein such as IgG faces also the same problem of poor mass transfer. Surface imprinting of IgG provide a route for protein imprinting but not satisfied for the huge proteins, the crosslinking degree need to be reduced further, but it normally leads also to poor recognition property of imprinted structure. Imprinting of IgG is very rewarding and need more investigations, since until right now most of protein imprintings were focused on Lyz, BSA, RNase, and HSA with the MW less than $70 \mathrm{kDa}$. Shea and his coworkers developed a new method to imprint IgG via hydrogel nanoparticle with multi functional groups complimentary to the IgG surface. Monomers with different functionalities such as NIPAAm, AA, N-(3-aminopropyl) methacrylamide hydrochloride, and N-tert-butyl acrylamide were used to form the imprinted structure based on scaffold imprinting, impressively, the degree of crosslinking is only $2 \%{ }^{[153]}$ In combination of scaffold imprinting and surface imprinting, imprinting for big size protein achieved in a low crosslinking degree is proposed a feasible way to imprint large size proteins. 


\subsection{SI-ATRP and subsequent hydrolysis on cellulose membrane}

\subsubsection{Characterization of cellulose base membrane}

TE PET 400 with isotropic pore structure is beneficial to characterize the modification via permporometry, contact angle, hydrodynamic layer thickness, but the relative small porosity and small specific surface resulted in relatively poor binding capacity and permeability, these limited the application of TE PET membrane as a membrane adsorber. In order to overcome such obstacles, regenerated cellulose membrane (R60, obtained from Whatman) was used as the new substrate. Cellulose is the most common organic polymer composed of $\beta-1,4-$ linked D-glucose (cf. Table 4.6). Regenerated cellulose membrane is made from the cellulose acetate via phase inversion and subsequent hydrolysis. Hydrophilic cellulose membrane has low nonspecific protein binding and reactive hydroxyl group on surface: therefore has been widely used for preparation of adsorptive membranes. ${ }^{[84]}$

At first the pore size distribution of the base cellulose membrane was measured by permporometry (Figure 4.43).

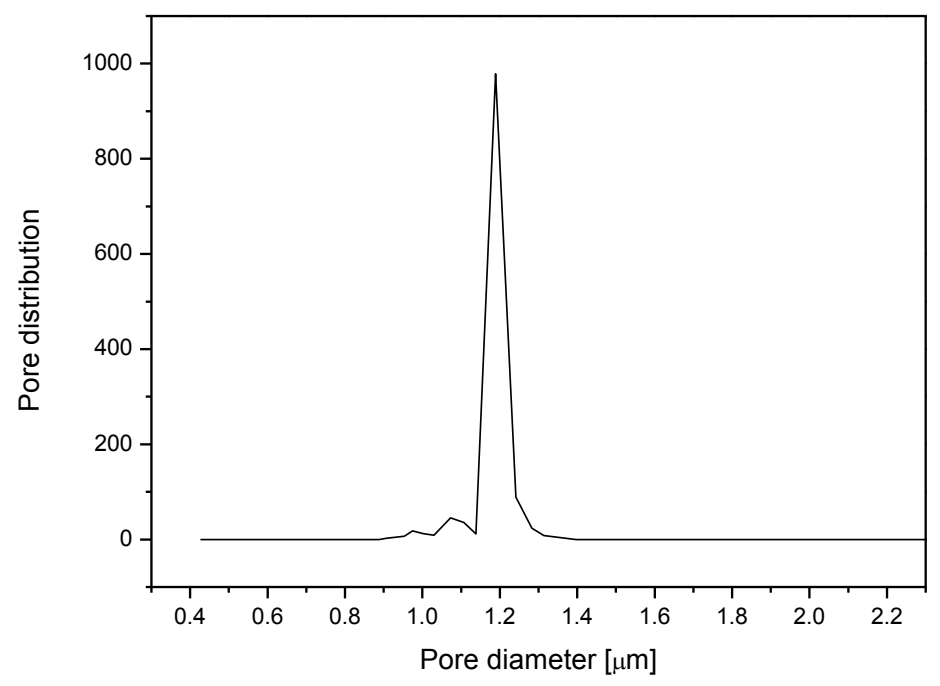

Figure 4.43 Pore size distribution measured by liquid dewetting permprometry for cellulose base membrane. 
The distribution of the barrier pore size is relatively narrow with diameter approximately 1.2 $\mu \mathrm{m}$ which was larger than the nominal diameter $1 \mu \mathrm{m}$ from the manufacturer. The pore structure of the cellulose membrane was observed by SEM (Figure 4.44).

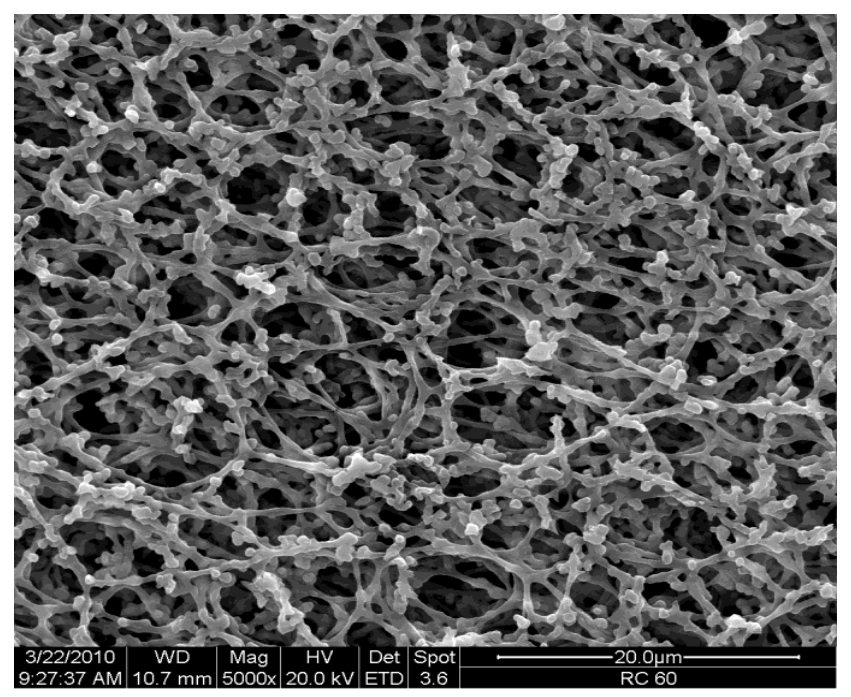

Figure 4.44 SEM image of base membrane

Due to the irregular pore shape, Hagen-Poiseuille model cannot be used for characterization of cellulose membrane's pore sructure. The specific surface area was determined by nitrogen adsorption / BET analysis as described in section 3.3.4. The specific surface of cellulose membrane with $6.51 \mathrm{~m}^{2} / \mathrm{g}$ was larger than that of PET 400 with $2.99 \mathrm{~m}^{2} / \mathrm{g}$. Cellulose membrane has thickness of $75 \mu \mathrm{m}$ and which for PET membrane is only $23 \mu \mathrm{m}$. At the same size (diameter $25 \mathrm{~mm}$ ), the weights of cellulose and PET membrane samples are similar in the range from 11.7 to $12.2 \mathrm{mg}$ for PET and 12.86 to $13.87 \mathrm{mg}$ for cellulose.

Table 4.6 Comparison of cellulose membrane and TE PET 400 membrane.

\begin{tabular}{|c|c|c|c|c|c|}
\hline & Chemical structure & Pore type & $\begin{array}{l}\text { Pore diameter } \\
{[\mathrm{nm}]}\end{array}$ & $\begin{array}{c}\text { Porosity } \\
\text { [\%] }\end{array}$ & $\begin{array}{c}\text { Specific surface area } \\
{\left[\mathrm{m}^{2} / \mathrm{g}\right]}\end{array}$ \\
\hline $\begin{array}{l}\text { TE PET } \\
400\end{array}$ & 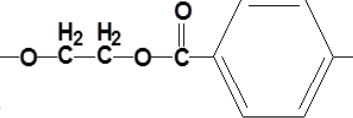 & $\begin{array}{l}\text { isotropic, } \\
\text { cylindrical }\end{array}$ & 675 & 10 & 2.99 \\
\hline $\begin{array}{l}\text { Cellulose } \\
\text { R60 }\end{array}$ & $\mathrm{OH}$ & irregular & 1200 & 60 & 6.51 \\
\hline
\end{tabular}




\subsubsection{Surface functionalization via initiator immobilization and subsequent ATRP}

Husson et al. had grafted PAA, ${ }^{[157]}$ PPEGMA, ${ }^{[158]}$ and PDEMAEMA ${ }^{[68]}$ via SI-ATRP on cellulose R60 membrane, the initiator was immobilized on cellulose surface in solvent THF. In this work, the immobilization in THF was first tested via variation of reaction time from 0 to $6 \mathrm{~h}$ and subsequently characterized via SI-ATRP. The result is shown in Figure 4.45.

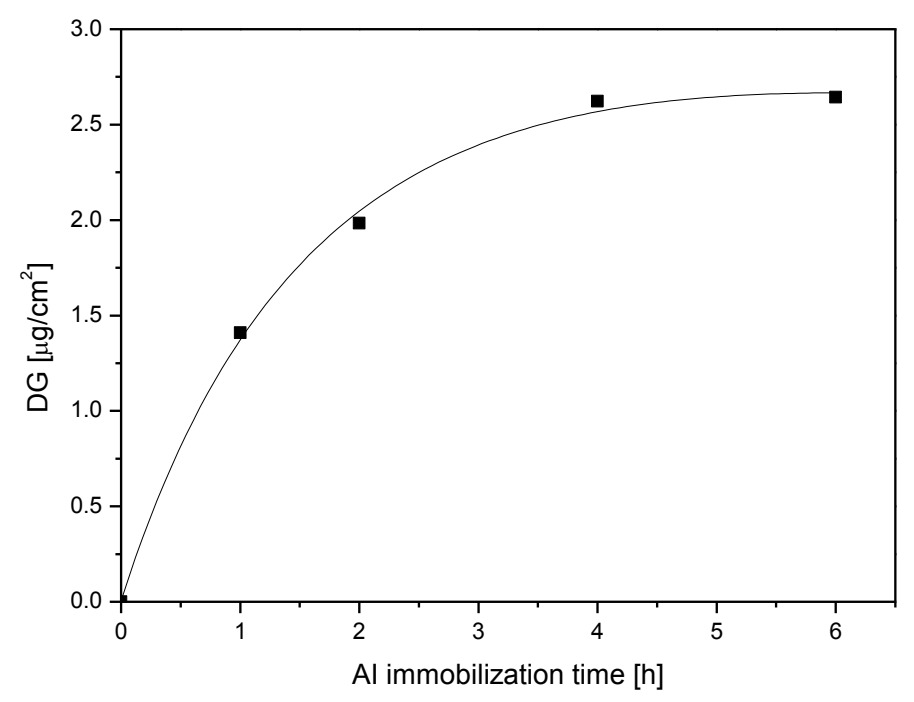

Figure 4.45 DG for PtBMA on cellulose membrane versus the Al immobilization time in THF, ATRP: $1.5 \mathrm{~mol} / \mathrm{L}$ tBMA solution in MeCN / water solution mixture (V:V=9:1) with a catalyst system of $[$ tBMA]:[CuCl]:[CuCl$]:[B p y]=75: 1: 0.1: 2.75$ at ambient temperature for $5 \mathrm{~h}$.

The conversion of the hydroxyl groups on cellulose membrane surface was improved by extension of the reaction and reached saturation till $4 \mathrm{~h}$. The specific surface area is 872.85 $\mathrm{cm}^{2}$ per membrane $(\varnothing 25 \mathrm{~mm}$ ), which was calculate from specific surface area of BET measurement and the average base cellulose membrane weight of $13.42 \mathrm{mg}$. The base cellulose membrane was very hydrophilic with a contact angle $0^{\circ}$. After initiator immobilization, surface was converted to very hydrophobic with a contact angle $136.4^{\circ}$ (Figure 4.51), which was higher than that of PET membrane after Al immobilization with $93.7^{\circ}$. The increased hydrophobicity of cellulose membrane may result from the higher bromide density in comparing with PET. Therefore, the reduction of the bromide was a potential requirement for imprinting on cellulose membrane, dilution of the bromide group on cellulose surface was carried out via mixture propionyl bromide and $\alpha$-bromoisobutyryl 
bromide $(\mathrm{BiB})$ in the reaction solvents of TFA and $\mathrm{MeCN}$ and subsequently characterized via ATRP of $t$ BMA (Figure 4.46).

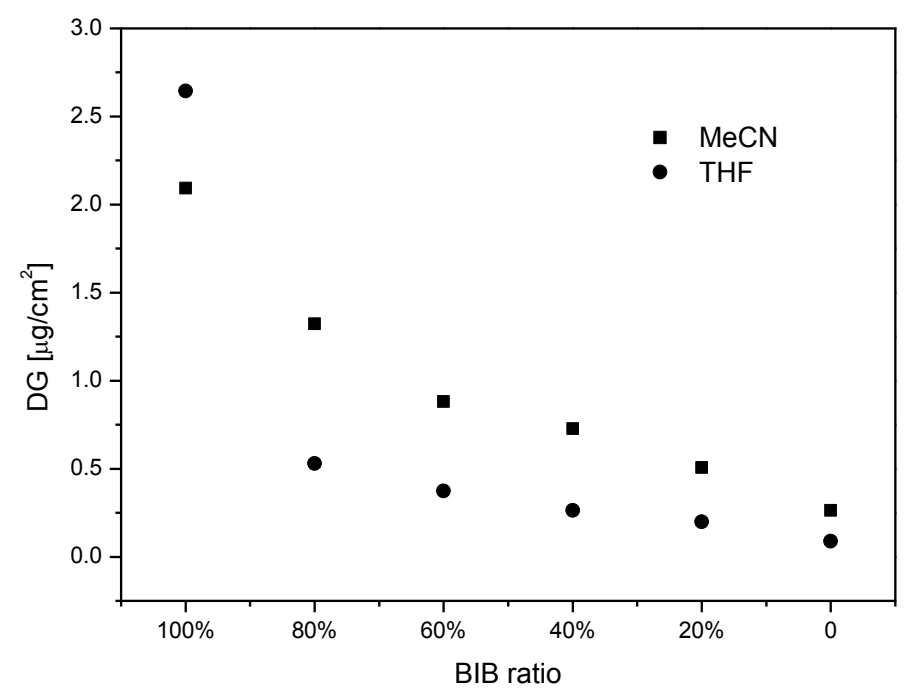

Figure 4.46 DG for PtBMA on cellulose membrane versus the ratio of $\alpha$-bromoisobutyryl bromide (BIB) in mixture of BIP and propionyl bromide in MeCN (squares) and THF (points), ATRP: $1.5 \mathrm{~mol} / \mathrm{L}$ tBMA solution in MeCN / water solution mixture (V:V = 9:1) with a catalyst system of $[$ tBMA]:[CuCl]:[CuCl 2$]:[\mathrm{Bpy}]=75: 1: 0.1: 2.75$ at ambient temperature for $5 \mathrm{~h}$.

The DG of PtBMA decreased with decreasing ratio of $\alpha$-bromoisobutyryl bromide. Below $80 \%$ ratio of $\alpha$-bromoisobutyryl bromide, the DGs from THF series were lower than those of $\mathrm{MeCN}$ series. The tendency in $\mathrm{MeCN}$ is smooth and demonstrated a good control of initiator dilution. There was a sharp jump of DG in THF series from $\alpha$-bromoisobutyryl bromide ratio $80 \%$ to $100 \%$ in comparing with the tendency by MeCN. Tomicki et al. have found the same DG jump phenomenon, when the BIB ratio was reduced from $100 \%$ to $50 \% .{ }^{[14]}$ Then MeCN was chosen as Al immobilization solvents, dilution of the initiator was feasible to control the grafted polymer density. Similar work had been done by Tomicki et al. on PET 400 membrane, the diluted bromide density was characterized successfully via XPS spectra. ${ }^{[14]}$

The kinetics of ATRP was characterized via variation of time after the initiator (100\% BIB) immobilization in MeCN (Figure 4.47). 


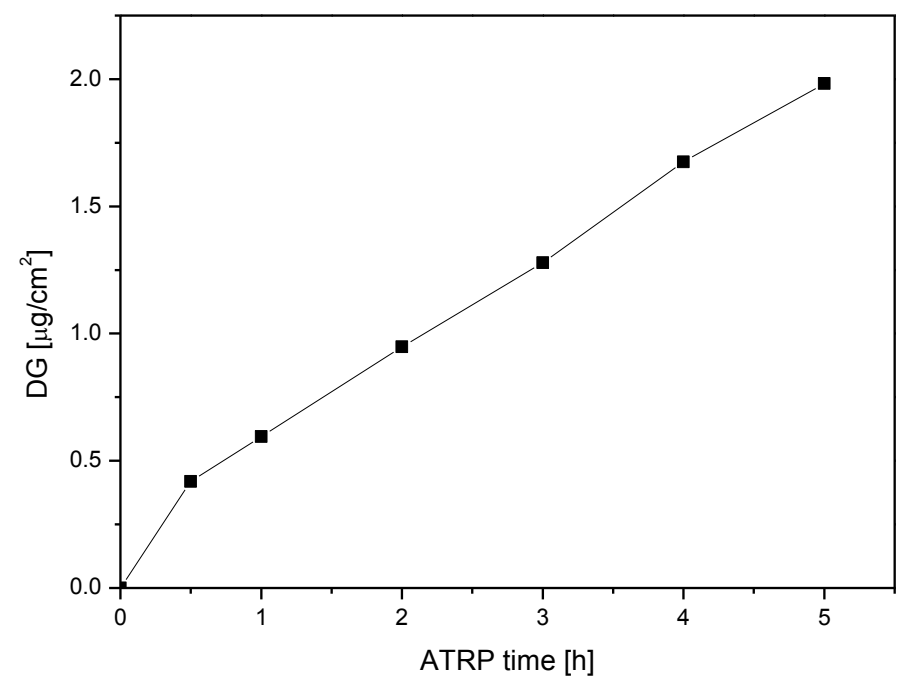

Figure 4.47 DG for PtBMA on cellulose membrane versus reaction time, ATRP: $1.5 \mathrm{~mol} / \mathrm{L}$ tBMA solution in $\mathrm{MeCN} /$ water solution mixture $(\mathrm{V}: \mathrm{V}=9: 1)$ with a catalyst system of $[t \mathrm{BMA}]:[\mathrm{CuCl}]:\left[\mathrm{CuCl}_{2}\right]:[\mathrm{Bpy}]=75: 1: 0.1: 2.75$ at ambient temperature .

There was a DG "jump" within the short reaction time of 30 min, which has been minimized in the ATRP on PET membrane under the same reaction condition (cf. Figure 4.18). More hydrophobic cellulose surface than the PET after initiator immobilization has proved by the contact angle measurement, it resulted from more bromide group on the cellulose membrane surface. High concentration of bromide is in favor for a quick initiation at the beginning of ATRP, which is demonstrated normally as a DG "jump" by SI-ATRP. From $1 \mathrm{~h}$ to $5 \mathrm{~h}$, the relationship was kept linear very well and further scaffold chain optimization via ATRP was not necessary (cf. Figure 4.18).

\subsubsection{Hydrolysis of PtBMA to PMAA on cellulose membrane.}

The hydrolysis of PtBMA to PMAA on cellulose membrane surface was investigated firstly in $7 \mathrm{~mol} / \mathrm{L} \mathrm{HCl}$ and 50\% TFA in MeCN / water solution mixture (V:V = 1:1) which had been shown as good conditions for the hydrolysis of PtBMA on PET membrane. However, PtBMAg-cellulose membrane dissolved in the $7 \mathrm{~mol} / \mathrm{L} \mathrm{HCl}$ and the $D_{\text {PMAA }}$ was negative in $50 \%$ TFA solution. 


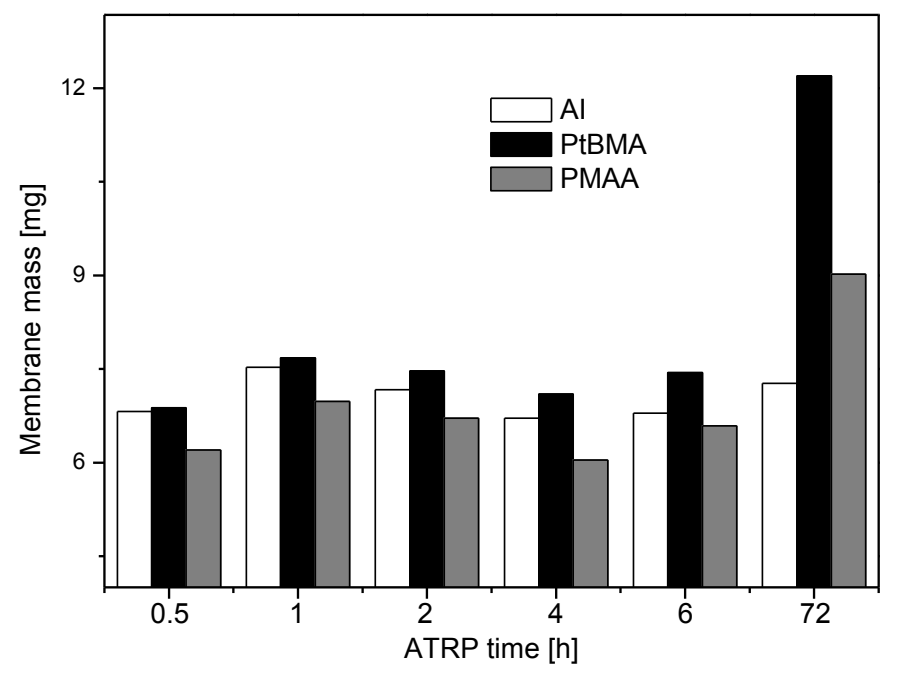

Figure 4.48 Mass of $\mathrm{Al}$ (open columns), PtBMA (black columns) and PMAA(gray columns) grafted cellulose membrane ( $\varnothing 18 \mathrm{~mm}$ ) versus ATRP reaction time, ATRP in $1.5 \mathrm{~mol} / \mathrm{L} t \mathrm{BMA}$ solution in $\mathrm{MeCN}$ / water solution mixture ( $\mathrm{V}: \mathrm{V}=9: 1)$ with a catalyst system of $[t \mathrm{BMA}]:[\mathrm{CuCl}]:\left[\mathrm{CuCl}_{2}\right]:[\mathrm{Bpy}]=75: 1: 0.1: 2.75$ at ambient temperature; hydrolysis of the PtBMA-g-cellulose membranes in $50 \%$ trifluoroacetic acid solution in $\mathrm{MeCN} /$ water mixture $(\mathrm{V}: \mathrm{V}=1: 1)$ at $50^{\circ} \mathrm{C}$ for $14 \mathrm{~h}$.

After hydrolysis, the membrane masses of PMAA-g-cellulose membranes were less than the corresponding Al membranes till $6 \mathrm{~h}$ ATRP of $t \mathrm{BMA}$. The cellulose membrane is also not stable in $50 \%$ trifluoroacetic acid solution. For $72 \mathrm{~h}$ ATRP and subsequent hydrolysis, the DG of PMAA is positive but still much less than the theoretical value. However, much better than the results of short reaction time samples. This indicated that the cellulose structure is more stable than PtBMA, main leakage of the hydrolyzed cellulose backbone was taken place after the complete hydrolysis of PtBMA. It seemed feasible to optimize the hydrolysis condition through reduction of TFA concentration to hydrolyze PtBMA selectively and meanwhile to better protect base cellulose membrane (Figure 4.50). 


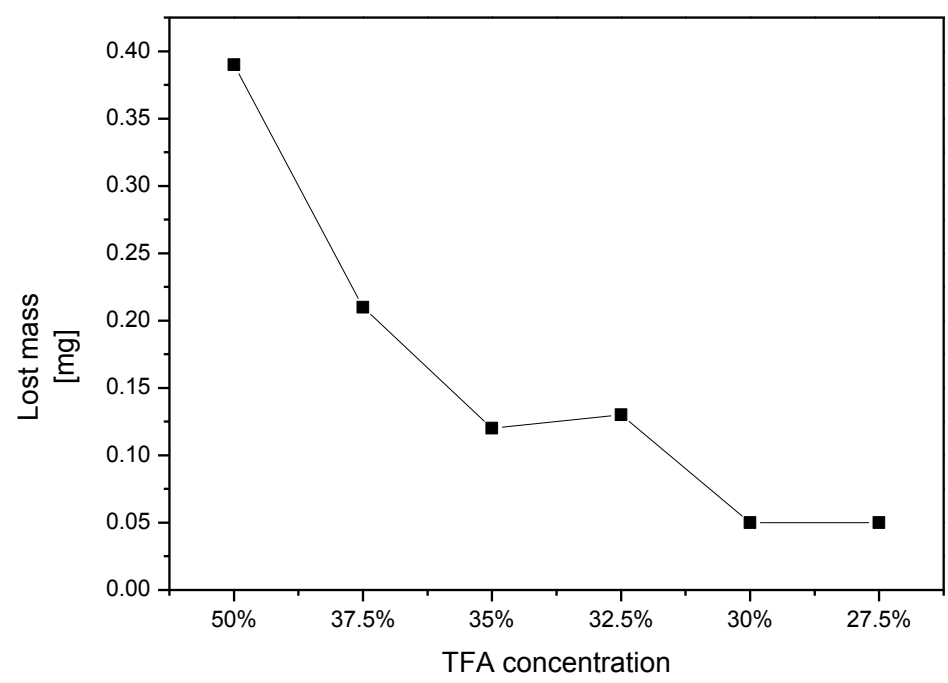

Figure 4.49 Lost mass of base cellulose membrane $(\varnothing 18 \mathrm{~mm})$ versus TFA concentration in $\mathrm{MeCN} /$ water solution mixture $(\mathrm{V}: \mathrm{V})=1: 1$ at $50^{\circ} \mathrm{C}$ for $14 \mathrm{~h}$.

The lost mass of base membrane decreased with the TFA concentration from $50 \%$ to $27.5 \%$. That lost mass in $32.5 \%$ TFA is more than that in 35\% TFA should be an experimental error. Base cellulose membrane demonstrated a good stability in low concentrations $30 \%$ and $27.5 \%$ of TFA with lost mass $0.05 \mathrm{mg}$. The ability of the solution with different TFA concentration for hydrolysis of PtBMA was further investigated via $\mathrm{CyC}$ adsorption and the results are shown in Figure 4.50.

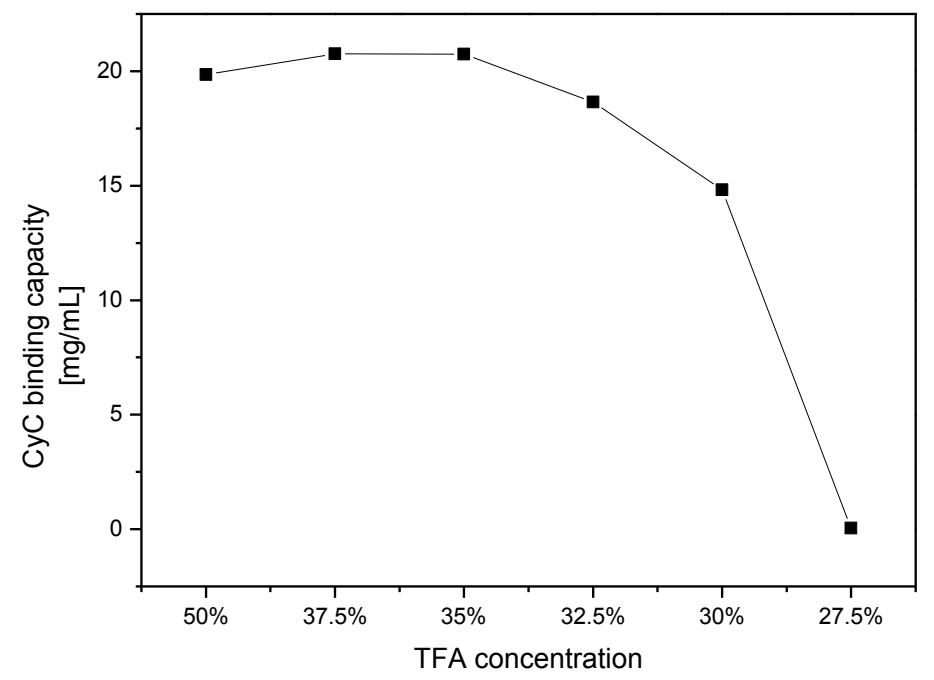


Figure 4.50 CyC binding capacity of PMAA-g- cellulose membrane versus TFA concentration in $\mathrm{MeCN} /$ water solution mixture $(\mathrm{V}: \mathrm{V}=1: 1)$ at $50{ }^{\circ} \mathrm{C}$ for $14 \mathrm{~h}$. ATRP in $1.5 \mathrm{~mol} / \mathrm{L} t \mathrm{BMA}$ solution in acetonitrile / water solution mixture ( $V: V=9: 1)$ with a catalyst system of $[t \mathrm{BMA}]:[\mathrm{CuCl}]:\left[\mathrm{CuCl}_{2}\right]:[\mathrm{Bpy}]=75: 1: 0.1: 2.75$ at ambient temperature for $5 \mathrm{~h} ; \mathrm{CyC}$ adsorption in $0.2 \mathrm{~g} / \mathrm{L}$ protein phosphate solution $(\mathrm{pH}=7.0,5 \mathrm{mmol} / \mathrm{L})$ for $2 \mathrm{~h}$.

The capacity of the PMAA-g-cellulose membrane increased by higher concentration of TFA and reached saturation at the $37.5 \%$, the slight reduction of the $\mathrm{CyC}$ capacity was due to hydrolysis of the backbone of cellulose what led to loss of the PMAA chains from membrane surface. The membrane hydrolyzed in $27.5 \%$ showed no CyC binding capacity and was difficult to wet in protein solution. Through characterization of contact angle, the surface was still hydrophobic with contact angle of $132^{\circ}$. It confirmed that the PtBMA has not been hydrolyzed. In consideration of the stability of base membrane, 35\% TFA was chosen for the further hydrolysis. After hydrolysis, surface became very hydrophilic again like base membrane with a contact angle 0 (Figure 4.51).

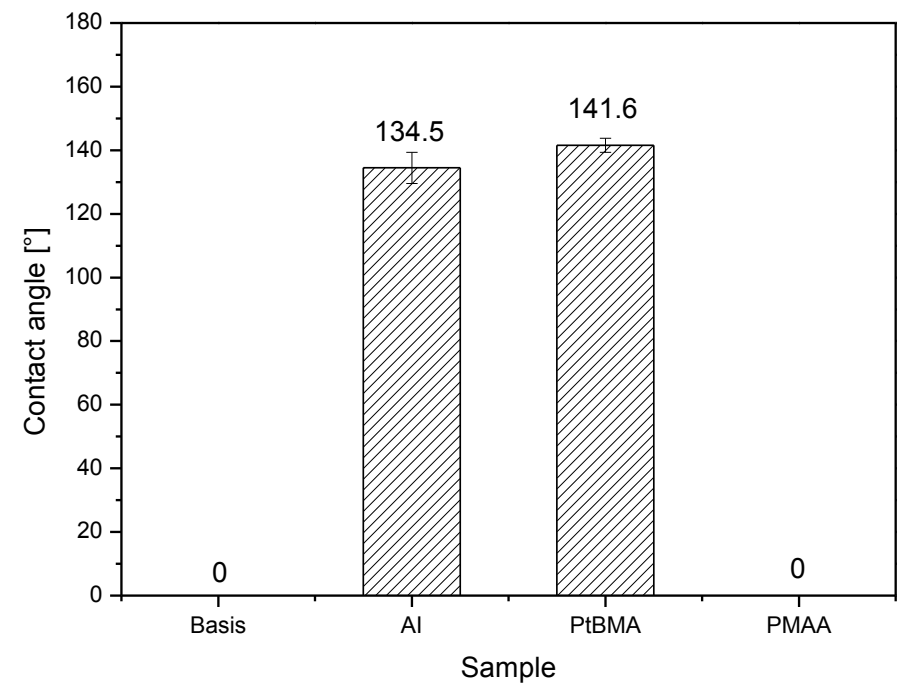

Figure 4.51 Contact angle results for membranes after different stages of functionalization. Al corresponds to a membrane after ATRP initiator immobilization; PtBMA and PMAA correspond to the membranes grafted with PtBMA (5 h ATRP) and PMAA (35\% TFA, $14 \mathrm{~h}$, $50^{\circ} \mathrm{C}$ ) respectively.

Base cellulose membrane was very hydrophilic. With the macroporous structure and high porosity, the drop of water can be absorbed into membrane immediately and shown a 
contact angle $0^{\circ}$. After ATRP initiator immobilization, surface became hydrophobic by cover with isopropyl bromide group, the contact angle increased from $0^{\circ}$ to $134.5^{\circ}$, which is higher the PET 400 membrane after ATRP initiator immobilization with contact angle $94^{\circ}$. From the chemical structure of cellulose, the density of hydroxyl group should higher than that of PET even after oxidative hydrolysis. The convention of hydroxyl to isopropyl bromide group led to the more hydrophobic surface, but it was just a qualitative analysis. The best method to compare the surface bromide group density is XPS. Grafting of tBMA on the ATRP immobilized cellulose changed the hydrophilicity not as much as by PET membrane, this resulted from the already very hydrophobic surface of cellulose after ATRP initiator immobilization with a contact angle slightly increased from $134.5^{\circ}$ to $141.6^{\circ}$. After hydrolysis, membrane became hydrophilic again and the contact angle was $0^{\circ}$.

High porosity and hydrophilicity of cellulose membrane led to a strong water binding tendency and this resulted in some problems during the weighing process after modification, especially for the environment with high humidity. To minimize the measuring error during weighing process, membranes were weight after 1 hour drying by $50{ }^{\circ} \mathrm{C}$ and storing in measuring room at temperature about $20{ }^{\circ} \mathrm{C}$ to get the saturation of water molecule adsorption for $10 \mathrm{~min}$. Due to the variation of humidity, the weight results were used to compare with each other in one series experiment. Characterization of the hydrolysis is difficult via the weight change, while the water adsorption ability has been changed significantly by the switch of hydrophobic surface to hydrophilic surface after hydrolysis and led to a significant weighing experimental error. 


\subsection{Lysozyme imprinting on cellulose membrane}

\subsubsection{Characterizations of UV reactivity of PMAA- $g$-cellulose membrane}

Similar to the Lyz imprinting on PET membrane, the reactivity PMAA-g-cellulose membrane was investigated to improve the control the PAAm gel growth for imprinting. The UVreactivity was characterized via variation of UV-irradiation time using $0.3 \mathrm{~mol} / \mathrm{L} \mathrm{AAm}$ and MBAA with degree of crosslinking $10 \%$, in order to obtain a similar grafting degree to the DG of PMAA, which has also been done before by Lyz imprinting on PET membrane. The result is shown in Figure 4.52.

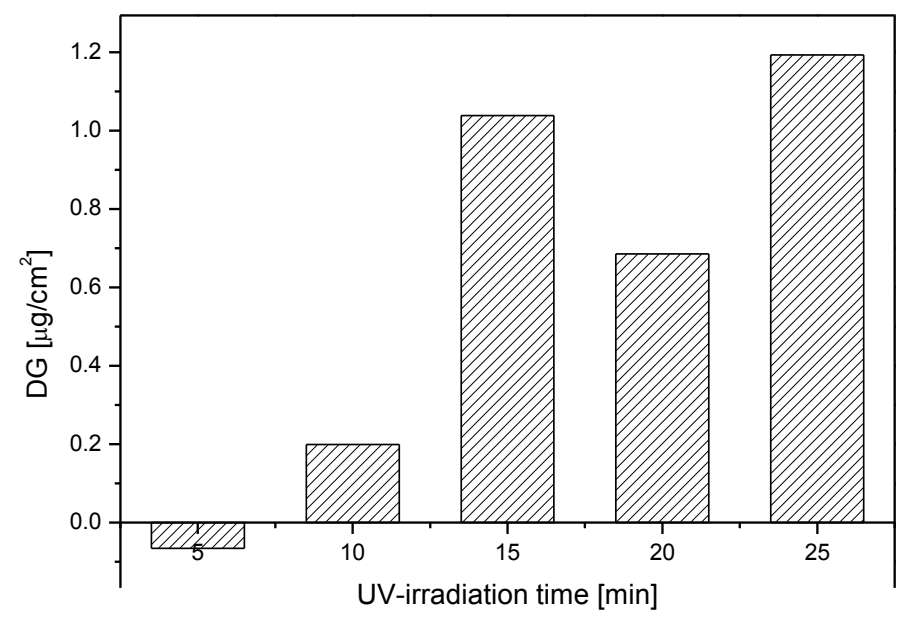

Figure 4.52 Degree of grafting for PAAm PMAA-g-cellulose membrane surface after ATRP (5 h) and hydrolysis versus the irradiation time: $0.3 \mathrm{~mol} / \mathrm{L}$ AAm and MBAA with degree of crosslinking 10\%, irradiation under the UV-intensity $24 \mathrm{~mW} / \mathrm{cm}^{2}$.

An increasing tendency has been observed, the negative DG range has shifted from 0-10 min to 0-5 min in comparing with the results on PET membrane (cf. Figure 4.26). This phenomenon resulted from the degassing process with argon to remove the oxygen (which was not done by imprinting on PET membrane) and the more bromide leading to the subsequent higher radical concentration via UV-initiation. For 25 min UV-irradiation time, the DG was about $1.2 \mu \mathrm{g} / \mathrm{cm}^{2}$, which was close to the theoretical DG of PMAA via calculation from the DG of PtBMA in Figure 4.47 (with $40 \%$ loss after hydrolysis). DG of membrane prepared under 20 min UV-irradiation was smaller than that of 15 min sample. This was due 
to the breaking of the membrane during post treatment resulted from the poor mechanical stability of the wetting cellulose membrane.

\subsubsection{Optimization of UV-irradiation time with $0.3 \mathrm{~mol} / \mathrm{L}$ monomer concentration}

Considering the possible high bromide group density, Lyz imprinting on PMAA-g-cellulose membrane ( via $3 \mathrm{~h}$ ATRP and hydrolysis ) was carried out in $0.3 \mathrm{~mol} / \mathrm{L}$ AAm and MBAA with degree of crosslinking $10 \%$ under UV- irradiation with intensity about $24 \mathrm{~mW} / \mathrm{cm}^{2}$ for 10 to 20 min (Figure 4.53).

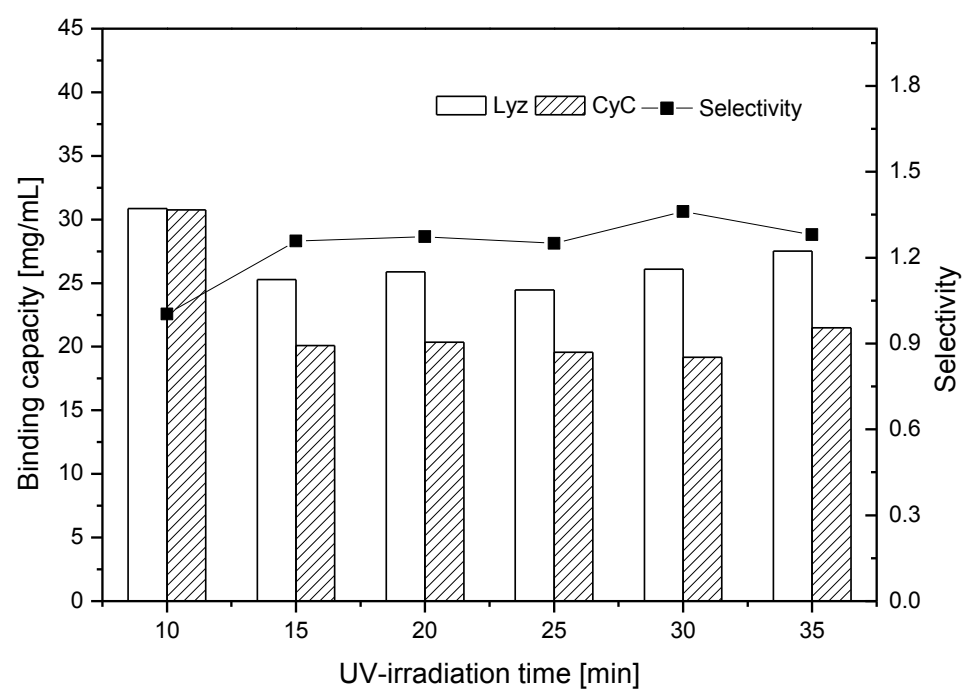

Figure 4.53 Influence of UV-irradiation time on the properties of Lyz-imprinted cellulose membranes: the Lyz and CyC binding capacities (open and solid columns) and resulting Lyz vs. CyC selectivity (squares) of the different MIP membranes with the same length of scaffold chains (ATRP 3 h; UV: $0.3 \mathrm{~mol} / \mathrm{L}$ functional monomer; AAm:MBAA = 9:1, i.e. degree of crosslinking 10\%; UV-intensity about $24 \mathrm{~mW} / \mathrm{cm}^{2}$ ).

The membrane after 10 min UV-irradiation had high capacity of Lyz and CyC about $30 \mathrm{mg} / \mathrm{mL}$ and showed no selectivity of Lyz to CyC. Membrane after 15 and 20 min UV-irradiation had lower capacities than that of $10 \mathrm{~min}$, but the selectivities were still very low in a range of 1.25 to 1.27. In order to improve the imprinting effect, extension of the UV-irradiation time to 35 min under the same UV-intensity was tested. The results in Figure $\mathbf{4 . 5 3}$ showed no significant improvement of the selectivity. The slight increase of Lyz binding capacity from 
irradiation time $25 \mathrm{~min}$ to $35 \mathrm{~min}$ was due to the heat produced by long time irradiation. The produced heat led to break the hydrogen bonds and electrostatic interactions between PMAA, protein, and PAAm, heat resulted in swelling of the polymer matrix, this was trend to produce more free space for protein binding and reduce the fraction of specific interactions. Due to the small differences of the Lyz binding capacity and selectivity of Lyz to CyC from 10 min to 35 min irradiation, there was not enough PAAm hydrogel to fill around the PMAA/Lyz complex. It seemed that extension of UV-irradiation time under UV-intensity of $24 \mathrm{~mW} / \mathrm{cm}^{2}$ was not an efficient way to improve the property of imprinted structure.

\subsubsection{Optimization of UV-monomer concentration}

In order to improve the property of the imprinted structure, the space recognition was investigated via improvement of UV-intensity and monomer concentration in respect to fill enough hydrogel around PMAA/Lyz complex (Figure 4.54).

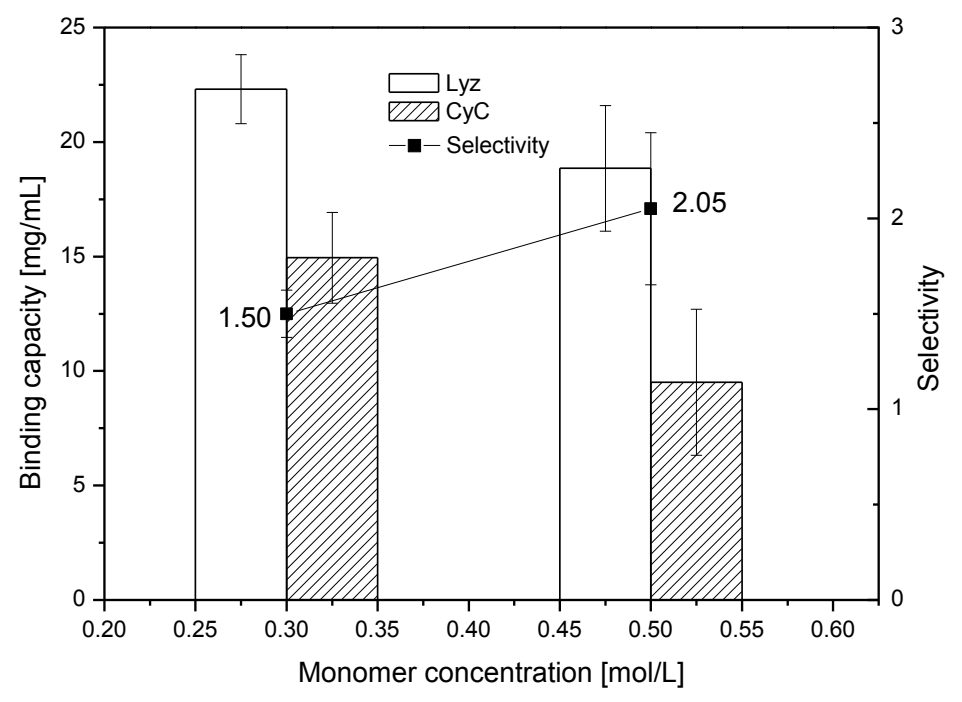

Figure 4.54 Influence of UV-monomer concentration on the properties of Lyz-imprinted cellulose membranes: the Lyz and CyC binding capacities (open and solid columns) and resulting Lyz vs. CyC selectivity (squares) of the different MIP membranes with the same length of scaffold chains (ATRP 3 h; UV: 0.3 and 0.5 mol/L functional monomer; AAm:MBAA = 9:1, i.e. degree of crosslinking 10\%; UV-intensity about $64 \mathrm{~mW} / \mathrm{cm}^{2} ; 12 \mathrm{~min}$ ). 
Using $0.5 \mathrm{~mol} / \mathrm{L} \mathrm{AAm}$ and MBAA instead of $0.3 \mathrm{~mol} / \mathrm{L}$, the imprinting effect was improved and demonstrated by the increase of selectivity from 1.50 to 2.05 under $64 \mathrm{~mW} / \mathrm{cm}^{2}$ irradiation. According to the result in Figure 4.25, the improved monomer concentration led more PAAm polymer around the PMAA/Lyz complexes after UV-irradiation, whereas the accessibility of protein was also reduced, it was confirmed by the reduction of Lyz binding capacity from 22.3 to $19.4 \mathrm{mg} / \mathrm{mL}$. The same improvement of imprinting property was achieved by increase of monomer concentration in other work, the separation of Lyz from BSA was improved in HPLC via preparation in a high monomer solution for imprinting. ${ }^{[49]}$ Monomer concentration larger than $0.5 \mathrm{~mol} / \mathrm{L}$ AAm and MBAA was not tested because of the limited solubility of MBAA. For the same concentration of $0.3 \mathrm{~mol} / \mathrm{L}$ functional monomer, the selectivity of Lyz to CyC was improved from 1.36 (cf. Figure 4.53) to 1.50 by the similar UV-irradiation dose via increasing the UV-intensity from $24 \mathrm{~mW} / \mathrm{cm}^{2}$ to $64 \mathrm{~mW} / \mathrm{cm}^{2}$. It is feasible to improve the imprinting effect via improvement of the UV-irradiation intensity.

\subsubsection{Optimization of UV-intensity}

Following the optimization of section 4.9.3, the further increase of AAm and MBA concentration was limited, the optimization was further tested via variation of UV-intensity to improve the property of imprinted structure. For the same UV-dose, UV-intensities of 14.5, 25.3 and $63.7 \mathrm{~mW} / \mathrm{cm}^{2}$ were obtained from UVA Cube 2000 and $71.3 \mathrm{~mW} / \mathrm{cm}^{2}$ from UVA Print 100-200 (Figure 4.55).

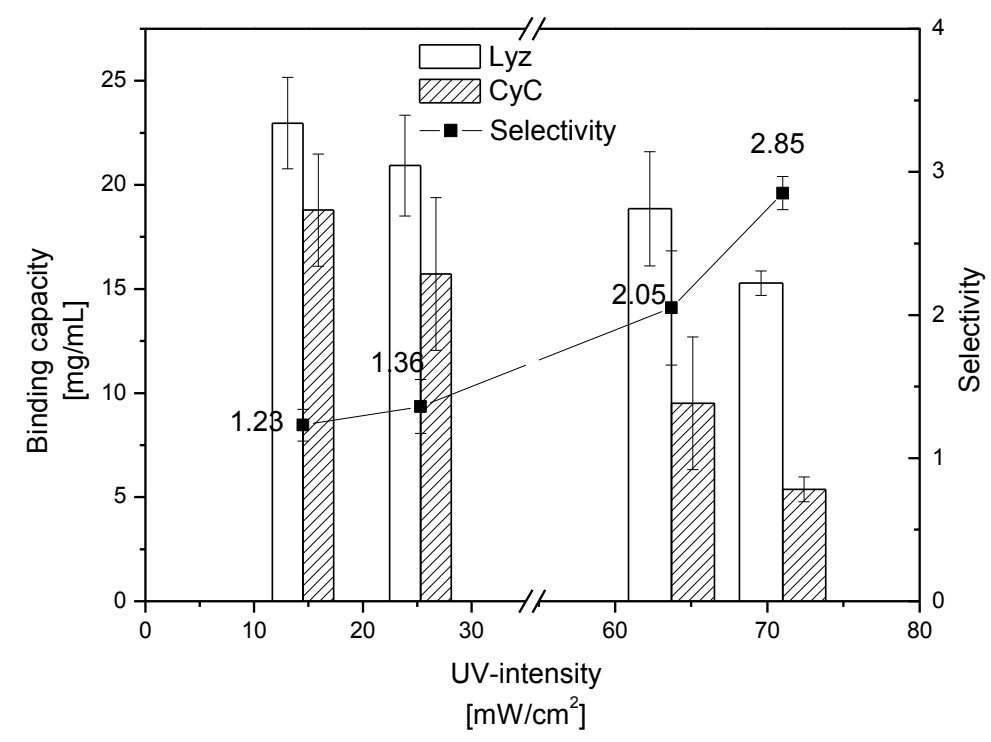


Figure 4.55 Influence of UV-intensity on the properties of Lyz-imprinted cellulose membranes: the Lyz and CyC binding capacities (open and solid columns) and resulting Lyz vs. CyC selectivity (squares) of the different MIP membranes with the same length of scaffold chains (ATRP $3 \mathrm{~h}$; UV: $0.5 \mathrm{~mol} / \mathrm{L}$ functional monomer; AAm:MBAA = 9:1, i.e. degree of crosslinking $10 \%)$.

Under the same UV-irradiation dose, the selectivity was increased from 1.23 to 2.85 by increasing of the UV-intensity from 14.5 to $71.3 \mathrm{~mW} / \mathrm{cm}^{2}$. Increase of UV-intensity had a similar effect as the improvement of monomer concentration by production of more PAAm polymer which was proved in section 4.5.1. The jump of selectivity from UV-intensity 63.7 to $71.3 \mathrm{~mW} / \mathrm{cm}^{2}$ is because the cooling system of UVA Cube 100-200 was worse than that of UVA Cube 2000. More heat transferred to the reaction solution in UVA Print 100-200 could accelerated the rate of the UV-grafting/crosslinking and produce more PAAm hydrogel around the PMAA/Lyz complexes. The decreasing tendency of Lyz binding capacity confirmed the existence of the more PAAm hydrogel which led a poor accessibility for protein rebinding. Therefore, UVA Print 100-200 with UV-intensity $71.3 \mathrm{~mW} / \mathrm{cm}^{2}$ was chosen for the further optimization.

\subsubsection{Optimization of UV-irradiation time under highest UV-intensity}

After variation of monomer concentration and UV-intensity, the imprinting was investigated via variation of UV-irradiation time under UV-intensity $71.3 \mathrm{~mW} / \mathrm{cm}^{2}$ (Figure 4.56).

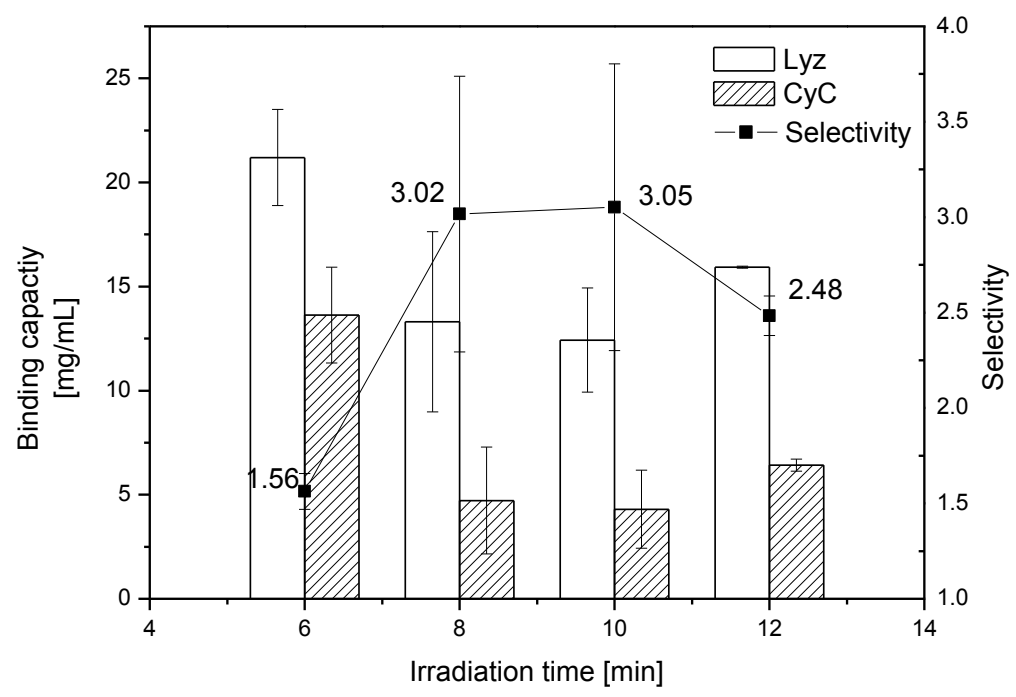


Figure 4.56 Influence of UV-irradiation time on the properties of Lys-imprinted cellulose membranes: the Lyz and CyC binding capacities (open and solid columns) and resulting Lyz vs. CyC selectivity (squares) of the different MIP membranes with the same length of scaffold chains (ATRP: 3 h; UV: $0.5 \mathrm{~mol} / \mathrm{L}$ functional monomer; AAm:MBAA = 9:1, i.e. degree of crosslinking 10\%; UV-intensity about $71.3 \mathrm{~mW} / \mathrm{cm}^{2}$ ).

The selectivity increased from 1.56 to 3.05 via extension of the UV-irradiation time from 6 $\min$ to $8 \mathrm{~min}$. Between 8 and $10 \mathrm{~min}$ irradiation time, the selectivity reached a plateau level of 3 and then reduced to 2.48 by extension of irradiation time from 10 to $12 \mathrm{~min}$. From 6 to $10 \mathrm{~min}$, the Lyz binding capacity was reduced from 21.2 to $12.4 \mathrm{mg} / \mathrm{mL}$ with is corresponding to the result in Figure 4.30, but selectivity decreased from 10 to $12 \mathrm{~min}$ UV-irradiation was different to the Lyz imprinting on PET membrane. The increased Lyz binding capacity from 10 to 12 min UV-irradiation was reverse to the argument which based on more PAAm hydrogel by longer irradiation time leading a lower Lyz binding capacity and high selectivity. In UVA Print 100-200, more heat was produced via UV-irradiation with influence on the conformation of the imprinted structure, which was considered as the main reason what led to the different phenomenon between results summarized in Figure 4.30 and Figure 4.56. The temperature in UVA Print 100-200 was measured after different irradiation times and is shown in Figure 4.57.

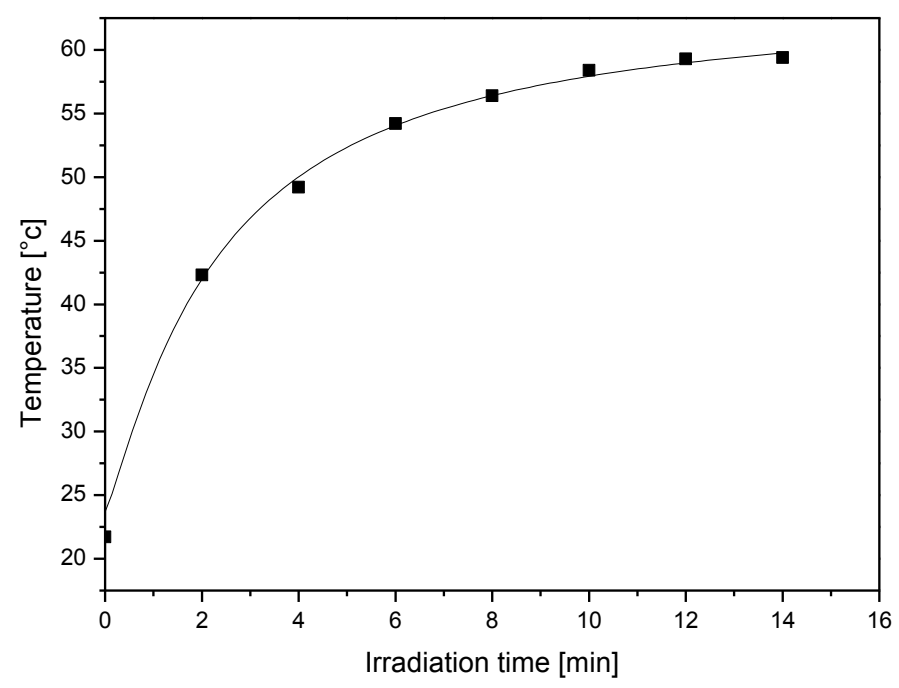

Figure 4.57 Temperature versus irradiation time under the UV-intensity about $71.3 \mathrm{~mW} / \mathrm{cm}^{2}$ in UVA Print 100-200. 
According to the work of Bloomfield and his coworkers, heat weakened the electrostatic interaction due to less favorable entropy and enthalpy. ${ }^{[159]}$ The pKa value of acid group is also shifted via temperature-induced reduction of the dielectric constant around the acid group in aqueous solution. ${ }^{[160]}$ The swelling of PAA increased by higher temperature was characterized by measuring the hydrodynamic particle diameter. ${ }^{[161]}$ The hydrogen bonds between carboxyl groups from PMAA and protein/amino groups from protein and PAAm can be also destroyed by higher temperature, which had been confirmed by measurement of the optical transmittance transition of $\mathrm{P}(\mathrm{AA}-\mathrm{CO}-\mathrm{AAm})$ hydrogel in aqueous solution. ${ }^{[162]}$ Hydrophobic effect was enhanced by increase of temperature, ${ }^{[159]}$ but the influence is limited during imprinting of Lyz by using hydrophilic PMAA and PAAm polymers in the very low salt concentration aqueous solution. Similar result has been observed by Zhao et al., the BSA imprinted hydrogel had higher binding capacity of BSA by increasing temperature for grafting/crosslinking. ${ }^{[163]}$ The weakening or breaking of electrostatic interaction and hydrogen bond led more swelling conformation of imprinted structure, which was benefit for more protein rebinding but with less fitting multi point interactions. More PAAm gel was filled in the PMAA/Lyz complex layer due to the increased temperature and produced more imprinted cavities with high accessibility, however, the recognition property of those imprinted cavities was low. Protein is thermo-sensitive, increase of temperature during imprinting process led more change of the protein conformation, which reduced the recognition property evaluated via rebinding at ambient temperature (about $20^{\circ} \mathrm{C}$ ).

Therefore, 10 min UV-irradiation (cf. Fig. 4.56) was chosen for the further optimization of binding specificity and selectivity.

\subsubsection{Optimization of crosslinking degree under highest UV-intensity}

After the optimizations of UV-intensity and UV-irradiation time, the improvement of imprinted property was further investigated via crosslinking degree variation, the crosslinking degree was varied from $10 \%$ to $15 \%$ which was the same range applied by Lyz imprinting on PET membrane. The results are shown in Figure 4.58. 


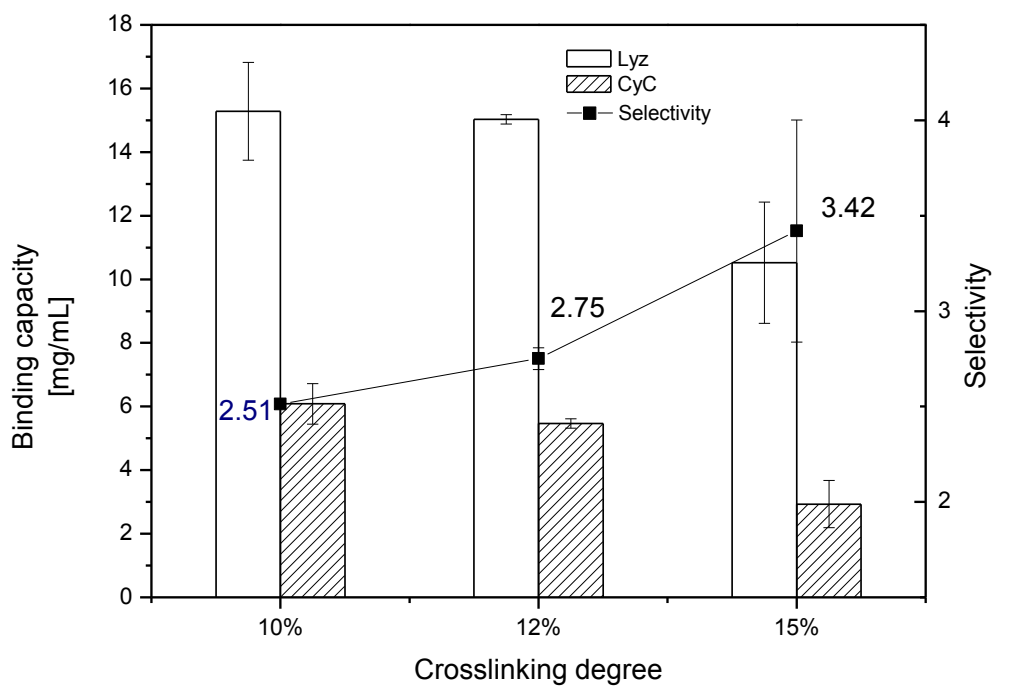

Figure 4.58 Influence of crosslinking degree on the properties of Lys-imprinted cellulose membranes: the Lyz and CyC binding capacities (open and solid columns) and resulting Lyz vs. CyC selectivity (squares) of the different MIP membranes with the same length of scaffold chains (ATRP: 3 h; UV: 0.5 mol/L functional monomer of AAm:MBAA; UV-intensity about 71.3 $\left.\mathrm{mW} / \mathrm{cm}^{2}, 10 \mathrm{~min}\right)$.

A maximum selectivity of 3.42 was obtained by shifting the crosslinking degree from $10 \%$ to $15 \%$. This has the same increasing tendency as the result of $D G=1.06 \mu \mathrm{g} / \mathrm{cm}^{2}$ series on PET membrane in Figure 4.32 via the same ATRP time of $3 \mathrm{~h}$ but leading to higher selectivity of 4.2. The reduction of the Lyz binding capacity in Figure 4.58 was $31 \%$ and much less than $74 \%$ in Figure 4.32. This resulted from more free space of the highly porous cellulose membrane in comparison with TE PET 400 membrane. More free space led to a less dense PAAm based gel which reduced the space imprinting effect and increased the accessibility for protein rebinding.

The experiment of Lyz imprinting was carried out in the UVA Cube 2000 under UV-intensity about $24 \mathrm{~mW} / \mathrm{cm}^{2}$ for $20 \mathrm{~min}$, this condition with better cooling system was proposed to produced less heat than that of the UVA Print 100-200 under UV-intensity about $71 \mathrm{~mW} / \mathrm{cm}^{2}$ for $10 \mathrm{~min}$, the produced heat led to a high Lyz binding capacity and low selectivity. In addition, the conformation of PMAA is thermosensitive as discussed in section 4.9.5, this is another reason to explain the result in Figure 4.58. After optimization of crosslinking degree, crosslinking degree $15 \%$ was chosen for the further optimization. 


\subsubsection{Optimization of UV-irradiation under highest UV-intensity with $15 \%$ degree}

\section{of crosslinking}

In UVA Print 100-200, the heat has played an important role on property of imprinted structure. The heat influence was investigated by the variation of the UV-irradiation time from 8 to 12 min with crosslinking degree = 15\% (Figure 4.59).

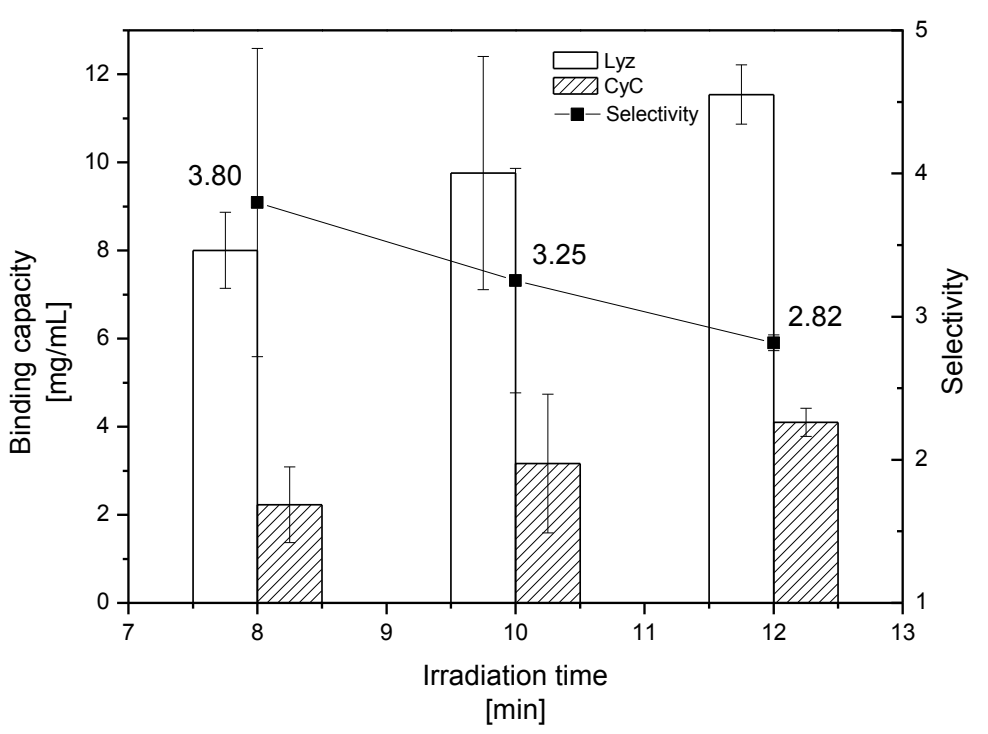

Figure 4.59 Influence of UV-irradiation time on the properties of Lys-imprinted cellulose membranes: the Lyz and CyC binding capacities (open and solid columns) and resulting Lyz vs. CyC selectivity (squares) of the different MIP membranes with the same length of scaffold chains (ATRP: 3 h; UV: 0.5 mol/L functional monomer of AAm:MBAA with crosslinking degree = 15\%; UV-intensity about $71.3 \mathrm{~mW} / \mathrm{cm}^{2}$ ).

The capacity of Lyz and CyC binding were increased from 8.0 to $11.5 \mathrm{mg} / \mathrm{mL}$ and 2.3 to 4.1 $\mathrm{mg} / \mathrm{mL}$, respectively. The selectivity of Lyz to CyC had a reducing tendency from 3.80 to 2.82 with the UV-irradiation time from 8 to $12 \mathrm{~min}$. It seemed that the heat effect leading high binding capacity and low selectivity had been enlarged by using high crosslinking degree $15 \%$, the selectivity reducing range shifted from 10-12 $\min$ to 8-12 $\mathrm{min}$ in comparison with the result of UV-irradiation time series of crosslinking degree $=10 \%$ in Figure 4.56. 8 min was best suited UV-irradiation time for preparation of imprinted PAAm based hydrogel. 


\subsubsection{Optimization of scaffold length}

Similar to the optimization of property of imprinted structure via variation of scaffold length on TE PET membrane, different DG of PMAA representing varied scaffold length had been obtained via variation of ATRP time from $1 \mathrm{~h}$ to $3 \mathrm{~h}$. DG of PMAA was calculated from the DG of PtBMA because of the weighing error before and after hydrolysis which has been discussed in section 4.8.3.

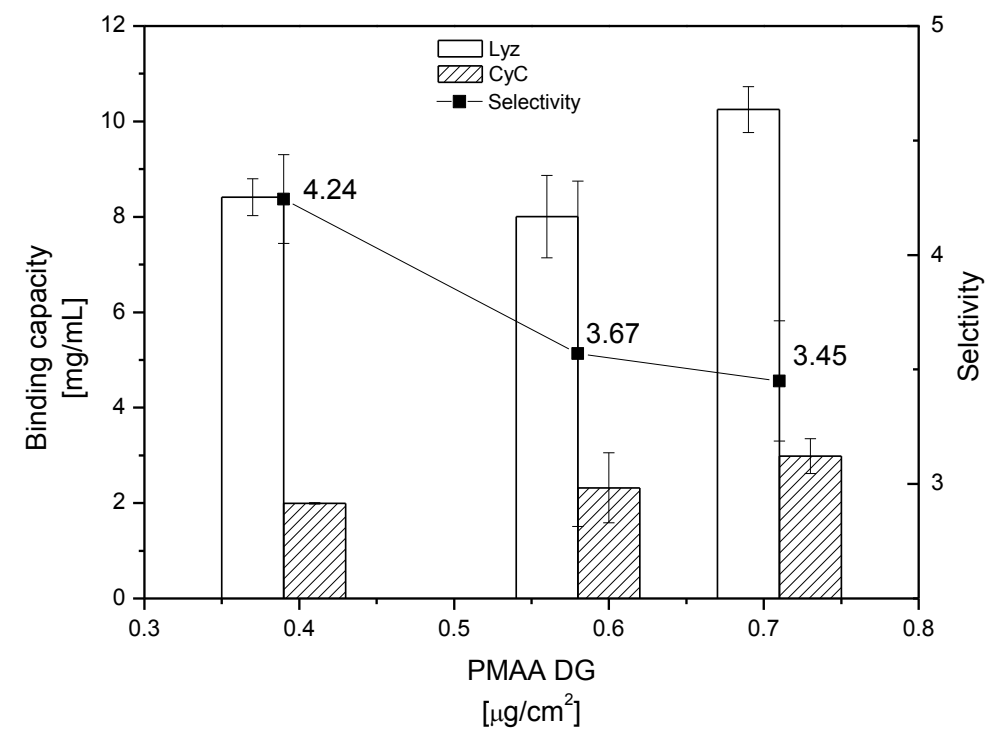

Figure 4.60 Influence of scaffold length on the properties of Lys-imprinted cellulose membranes: the Lyz and CyC binding capacities (open and solid columns) and resulting Lyz vs. $\mathrm{CyC}$ selectivity (squares) of the different MIP membranes with the same length of scaffold chains (ATRP: 1 to $3 \mathrm{~h}$; UV: $0.5 \mathrm{~mol} / \mathrm{L}$ functional monomer of AAm:MBAA with crosslinking degree = 15\%; UV-intensity about $71.3 \mathrm{~mW} / \mathrm{cm}^{2}$, UV-irradiation time $8 \mathrm{~min}$ ).

The capacities of Lyz and CyC showed the same tendency as scaffold length, the Lyz capacity of sample via $1 \mathrm{~h}$ ATRP was slightly higher than that of sample with $2 \mathrm{~h}$ ATRP, this resulted from experimental error; sample prepared via $2 \mathrm{~h}$ ATRP had a bigger error than the $1 \mathrm{~h}$ ATRP sample, especially for the selectivity. The tendency of selectivity was corresponding to the scaffold optimization on TE PET membrane. Longer scaffold adsorbed more protein and increased the protein-protein interaction and non-specific interaction between protein and scaffold, which led to reduced solvated protein surface and reduced the recognition property after imprinting. Short length of scaffold led to a lower protein binding capacity, the adsorbed proteins located in the near of the surface covered with UV-initiator bromide, those is favor to produce a better imprinted structure in consideration of the space effect by 
surface imprinting. It has been discussed by Ulbricht and his coworkers that the surface imprinting of 1,3,5-triazine herbicide desmetryn via photoinitiation of benzoin ethyl ether on PVDF membrane. ${ }^{[126]}$ The proteins immobilized on top of the PMAA/protein layer by long scaffold had long distance to the membrane surface. Most initiator groups for UV-grafting /crosslinking were located on the membrane surface (bottom of PMAA/protein layer) than located on or near surface of the PMAA/protein layer. UV-grafting/crosslinking on or near surface of PMAA/protein layer with less initiators lead to form less crosslinked polymer hydrogel and produced the imprinted structure with lower affinity to template. ${ }^{[64]}$

\subsubsection{Separation of protein mixtures}

Separation of protein mixture is an important unit in down stream process for protein production. Lyz and CyC are difficult to separate via the conventional technologies due to their similar size and isoelectric point (cf. Table 4.4). The Lyz selectivity of the MIP membrane was further investigated in a $0.2 \mathrm{~g} / \mathrm{L}$ protein mixture solution of Lyz and CyC (1:1). The adsorbed amounts of Lyz and CyC were determined by the enzyme assay with Micrococcus lysodeikticus and direct UV-Vis spectroscopy, respectively (cf. section 3.3.8). The results are shown below (Figure 4.61).

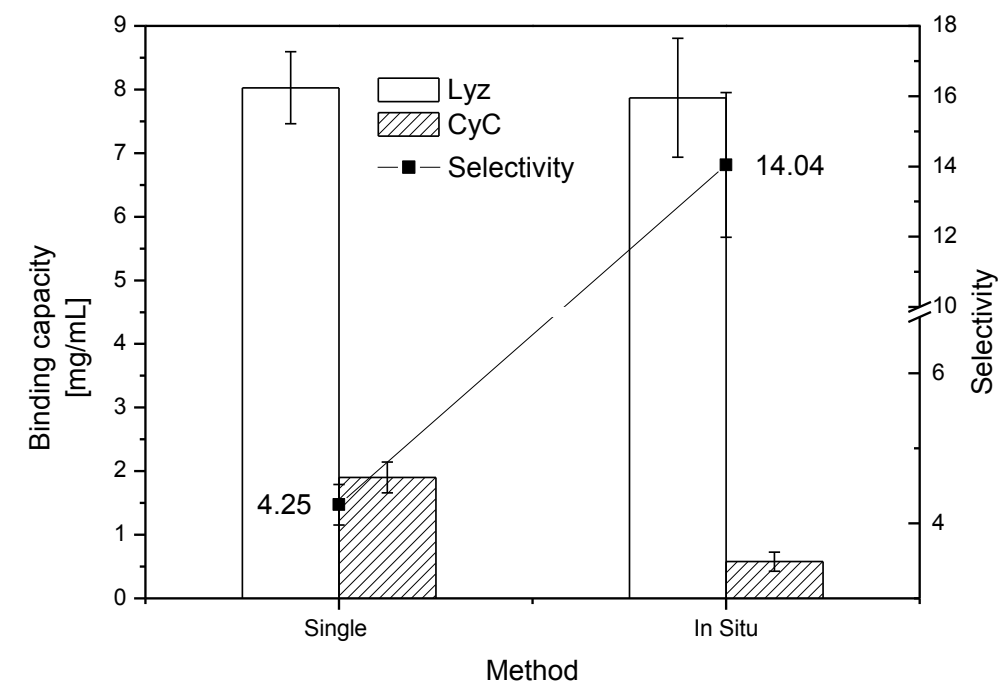

Figure 4.61 Comparison of single protein adsoption and in situ separation of protein mixture.

The recognition property was observed much more clearly by in situ experiment than two individual protein experiments. The selectivity of in situ experiment was 14.0, much higher 
than that from the two single protein experiments with 4.25. The Lyz binding capacity was only slightly reduced from 8.0 to $7.8 \mathrm{mg} / \mathrm{mL}$ from single protein experiment to in situ experiment, however, the $\mathrm{CyC}$ binding capacity in situ experiment is only $0.6 \mathrm{mg} / \mathrm{mL}$ and much less than $1.9 \mathrm{mg} / \mathrm{mL}$ by single $\mathrm{CyC}$ adsorption experiment. Adsorption in protein mixture is a competitive process, the imprinted cavities discriminated the bound protein not simply on based the size and charge, but on the synergistic effect of size, shape and arrangement of the multifunctional groups complementary to the imprinted template via prepolymerization (complexation) and subsequent crosslinking. The result of in situ experiment confirmed that the imprinted cavities have been successfully formed with specificity to the template Lyz. During the competitive adsorption of Lyz and CyC, the Lyz imprinted sites preferred to be occupied by Lyz and reduced the binding of CyC.

The composition of the elute (the concentration of the protein was about $30 \mathrm{mg} / \mathrm{L}$ ) from in situ experiment was also indentified by UV-Vis spectra in comparison with the pure Lyz and CyC phosphate buffer solutions with concentration $50 \mathrm{mg} / \mathrm{L}$.

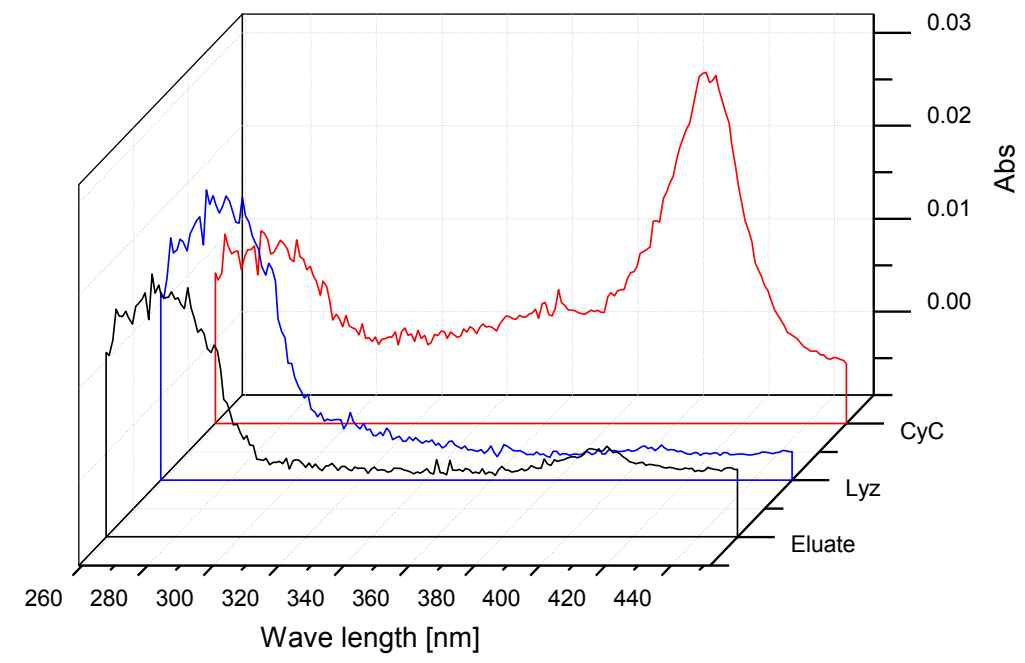

Figure 4.62 UV-Vis spectra of eluate from in situ experiment (black curve), and the pure Lyz (blue curve) and CyC (red curve) phosphate buffer solutions with concentration $50 \mathrm{mg} / \mathrm{L}$.

Both Lyz and CyC had absorption at the wavelength around $280 \mathrm{~nm}$, but only CyC has absorption at the wavelength around $410 \mathrm{~nm}$ and this is very significant. As shown in Figure 4.62, the eluate had strong absorption like single Lyz solution around $280 \mathrm{~nm}$, but the absorption peak around $410 \mathrm{~nm}$ for $\mathrm{CyC}$ was very weak. This is corresponding to the content 
determination by reaction between Micrococcus lysodeikticus and Lyz for Lyz analysis and UV-Vis spectra for CyC analysis.

\subsubsection{Comparison of MIP, NIP and scaffold membranes}

Through the above optimization, the imprinted structure showed a high binding of Lyz compared to competitive protein $\mathrm{CyC}$. The imprinting effect was further characterized in comparison of MIP, NIP and Scaffold grafted membrane (Figure 4.63).

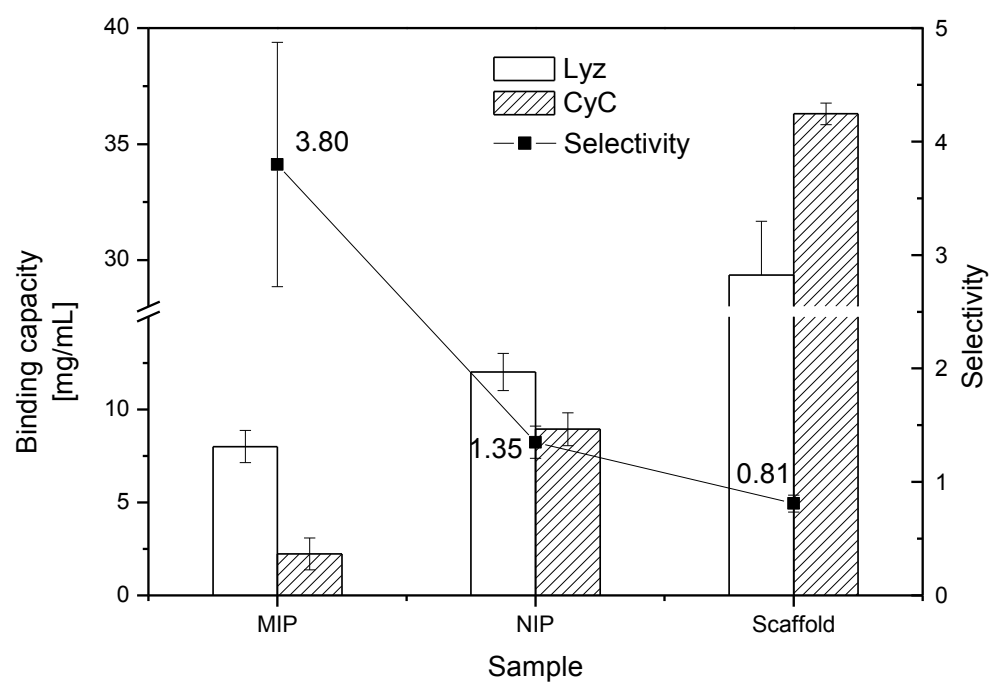

Figure 4.63 Lyz (open columns) and CyC (solid columns) binding capacities and Lyz to CyC selectivities (squares) for MIP, NIP and scaffold membranes (ATRP: 1 to h; UV: $0.5 \mathrm{~mol} / \mathrm{L}$ functional monomer of AAm:MBAA with crosslinking degree $=15 \%$; UV-intensity about 68 $\left.\mathrm{mW} / \mathrm{cm}^{2}, 8 \mathrm{~min}\right)$.

The scaffold membrane had a higher CyC binding capacity of $36.3 \mathrm{mg} / \mathrm{mL}$ than Lyz of 29.4 $\mathrm{mg} / \mathrm{mL}$, and no selectivity of Lyz to CyC was demonstrated with 0.81 . The isoelectric point and the size of protein should be considered. By the non-specific interactions based mainly on electrostatic interaction between PMAA and protein on cellulose membrane surface with less chain density or more free space, less negative charge CyC (cf. Table 4.4) can penetrate deeper into the less dens PMAA layer on cellulose membrane than Lyz. This is different to the result of PET in Figure 4.35, where the content of surface ariginine played dominate role for the protein adsorption, Lyz with $61 \%$ charge arginienes is more than that of CyC with $11 \%,{ }^{[12,165]}$ and scaffold on PET membrane has higher binding capacity of Lyz than CyC. The 
NIP showed a higher affinity to Lyz than CyC with the additional the hydrogen bond between protein and PAAm, the binding capacities of Lyz and CyC were reduced to 12.0 and 8.8 $\mathrm{mg} / \mathrm{mL}$ after grafting/crosslinking, but they were higher than those of MIP. This phenomenon resulted from highly porous and hydrophilic cellulose membrane. The imprinting effect was proved by the higher selectivity 3.8 of Lyz to CyC for MIP than that 1.4 of NIP. Before the crosslinking process, the presence of template protein led to a prepolymerization complex of the functional groups from scaffold PMAA, monomer AAm, and template protein in order to maximize the favorable interactions with the protein and formed stable complexes. After UV-initiated polymerization and template elution, the cavities with complementary size, shape and functional groups arrangement to template protein were left in the crosslinked hydrogel layer. However, the binding protein capacity of imprinted cellulose was much higher than imprinted PET membrane, when unit is (mass of adsorbed protein) / (mass of membrane). With same sample diameter, cellulose has similar weight to PET but the membrane volume is almost 3 times bigger than that of PET membrane or the surface area is more the 2 times bigger than that of PET( cf. Table 4.6). Overall, protein imprinted cellulose membrane has a higher performance than imprinted PET membrane.

\subsubsection{Protein binding isotherms of MIP and NIP membranes}

For better understanding of the specificity and affinity of imprinted structure, Lyz binding isotherms of MIP and NIP cellulose membrane was measured and fitted to the Langmiur module, results are shown in Figure 4.65. 


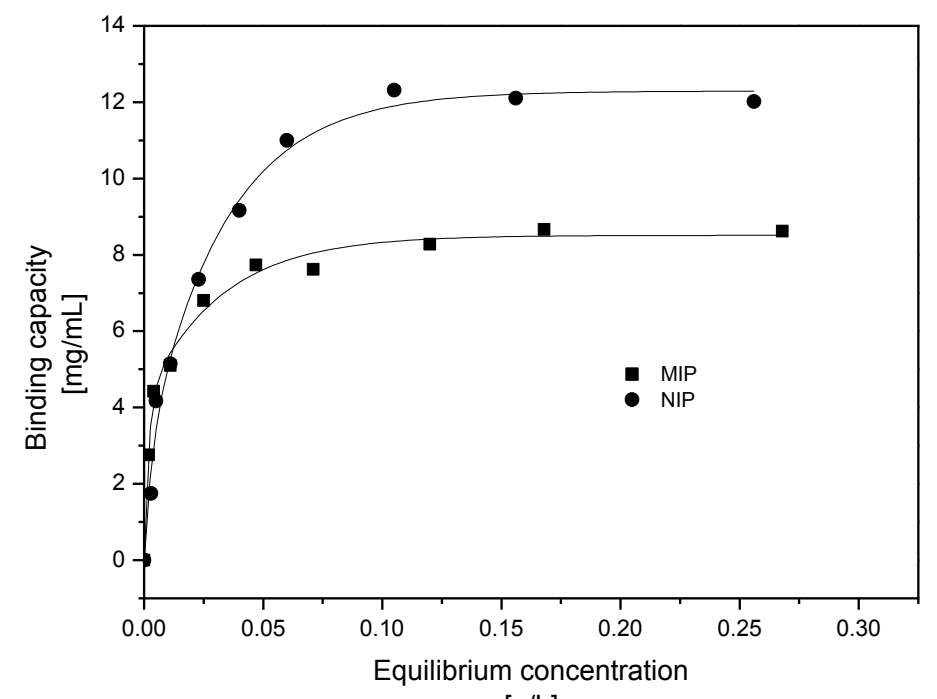

$[\mathrm{g} / \mathrm{L}]$

Figure 4.64 Lyz binding isotherms of MIP (solid squares) and NIP (solid circles) cellulose membranes as well as curves obtained by fitting the data to the Langmuir model.

The results showed that the NIP membrane had higher Lyz binding capacity of maximum $12.3 \mathrm{mg} / \mathrm{mL}$, which of MIP was $8.6 \mathrm{mg} / \mathrm{mL}$. This is corresponding to the result in Figure 4.63. Till equilibrium concentration of Lyz to $0.005 \mathrm{~g} / \mathrm{L}$, MIP membrane exhibited a steeper slope of adsorption capacity curve than that of NIP membrane. MIP membrane reached the saturation at equilibrium concentration $0.05 \mathrm{~g} / \mathrm{L}$, which is also lower than that of NIP membrane at $0.12 \mathrm{~g} / \mathrm{L}$. The protein binding affinity was characterized through calculating the binding constant $\left(\mathrm{K}_{\mathrm{L}}\right)$ in the frame of the Langmuir model. $\mathrm{K}_{\mathrm{L}}$ for Lyz binding to the MIP membrane was $258.6 \mathrm{~mL} / \mathrm{mg}$ (corresponding to $3.6^{*} 10^{7} \mathrm{~L} / \mathrm{mol}$ ). The binding of Lyz on NIP membrane was significantly less favorable with a $\mathrm{K}_{\mathrm{L}}$ of $75.6 \mathrm{~mL} / \mathrm{mg}\left(1.0 * 10^{7} \mathrm{~L} / \mathrm{mol}\right)$. In comparison with the $K_{L}$ of Lyz imprinting on PET membrane with $599 \mathrm{~mL} / \mathrm{mg}$, the affinity of the Lyz imprinting on cellulose membrane was lower than that of imprinting on PET membrane. The highly porous and hydrophilic substrate cellulose membrane, the harsh imprinting condition under high UV-intensity, and produced heat were probably the main reasons leading a lower affinity of the imprinted structure to template protein.

According the above experiments, the Lyz imprinting was transferred successfully from TE PET membrane surface to cellulose surface, although the chemical and physical properties of these two kinds of membranes are much different. Optimization techniques such as variations of UV-irradiation time, crosslinking degree, and scaffold length which has been 
developed for imprinting on TE PET membrane were also valid for imprinting on cellulose membrane. The poor mechanical of property of modified cellulose is an obvious limitation for its application. Combining with a stable substrate without reduction of the positive surface property of cellulose membrane is a promising way to overcome the above problem, such as Sartobind cellulose membrane from Sartorius which has been investigated by Ulbricht and his coworkers for preparation of membrane adsorbers for selective protein adsorption. ${ }^{[166]}$ 


\subsection{TE PET membrane immobilized with two "orthogonal" initiators}

Living radical polymerization demonstrated more precise control on the polymer structure in comparison with the traditional radical polymerization and has in the meantime been widely used for the small organic molecule template imprinting. For instance, it is possible to reduce the heterogeneity of the imprinted cavities in terms of morphology and binding affinity distribution. ${ }^{[167]}$ However, most protein imprinting is based on typical radical polymerization. In previous work described in sections 4.6 to 4.8 , living polymerization ATRP has demonstrated a precise control of scaffold and improved the property of imprinted membranes successfully. As shown in Figure 4.36, Crosslinking via conventional UV-initiated radical polymerization would lead to a high heterogeneity of the imprinted sites, therefore introduce a wide affinity distribution. In order to minimize the heterogeneity, living polymerization RAFT was planned to be applied for the crosslinking with expectation of better control. Zhang et al. have combined RAFT with protein imprinting. RAFT has improved the property of imprinted polystyrene bead with much higher imprinting factor of 12.7 and higher selectivity of more than 12 , while imprinting via typical radial polymerization with imprinting factor of 2.6 and almost no selectivity. ${ }^{[33,168]}$

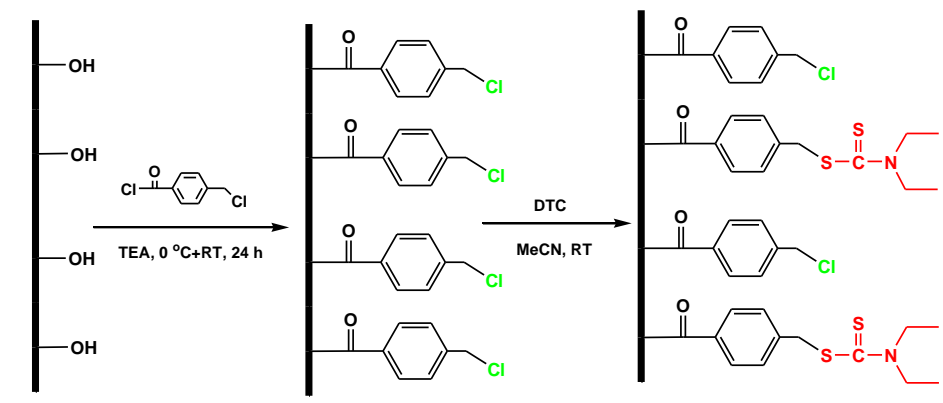

Scheme 4.3 Surface with two initiators: benzyl chloride for ATRP and DTC for UV-initiation (RAFT).

New strategy was utilized for immobilization of two different initiators on membrane surface for scaffold grafting and crosslinking via ATRP and UV-initiation of RAFT polymerization independently (Scheme 4.4). The carbon-bromide as ATRP initiator with UV-reactivity was replaced by carbon-chloride, and DTC was subsequently immobilized via substitution of chloride for UV-grafting/crosslinking, similar to the work of Sellergren and coworkers. ${ }^{[169]}$ 


\subsubsection{Immobilization of benzyl chloride group and subsequent characterization}

via ATRP

Because the 4-chlorobenzyl chloride is much more reactive than $\alpha$-bromisobutyrylbromide (for ATRP initiator immobilization procedure), chloride was added to the reaction solution drop wise at $0{ }^{\circ} \mathrm{C}$ without catalyst DMAP for the immobilization on PET membrane after amination. To improve the conversion of chloride, the reaction time was extended to $24 \mathrm{~h}$ at room temperature. After benzyl chloride immobilization, membrane surface became hydrophobic with contact angle $98.3^{\circ}$. At first, the reactivity of chloride was tested in $1.5 \mathrm{M}$ tBMA actonitrile and water (volume ratio 9:1) with a catalyst system [tBMA]:[CuCl]:[ Bpy]=100:1:2.5, which has successfully grafted PtBMA on PET membrane immobilized with isobutyryl bromide as ATRP initiator. After 6 hours reaction, almost no change of the membrane mass was observed. This resulted from the less ATRP reactivity of benzyl chloride with activation rate constant $k_{a c t}=5 * 10^{-3}$ than that of isobutyryl bromide with $k_{a c t}=2.7$. To improve the reactivity, PMDETA ( $\left.k_{a c t}=2.7\right)$ with was used to instead of Bpy $\left(k_{\mathrm{act}}=0.066\right)$, the result is shown in Figure 4.65.

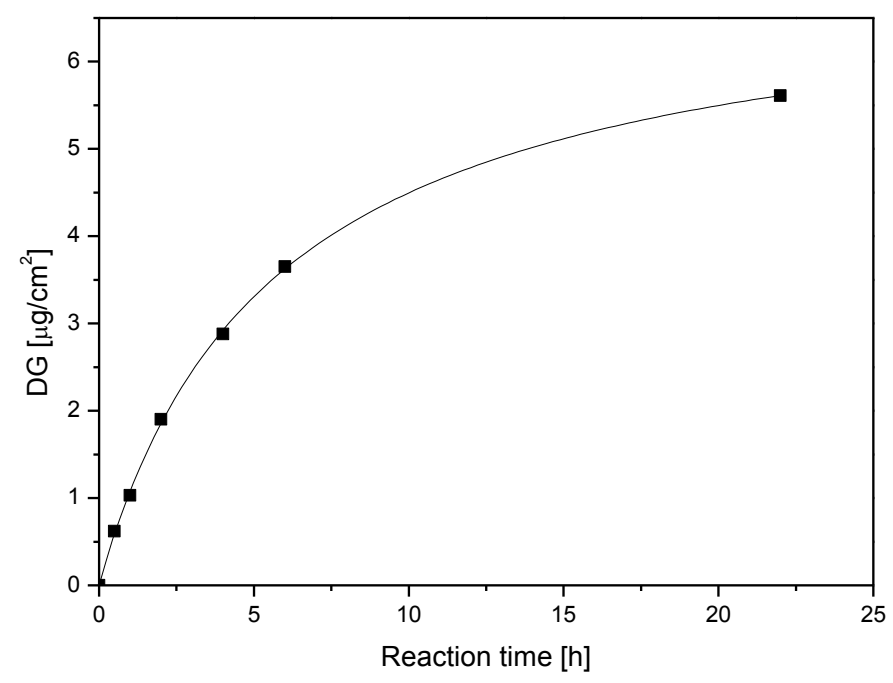

Fgirue 4.65 Degree of grafting for PtBMA on PET membrane surface with immobilized benzyl chloride (squares) versus the reaction time of ATRP in $1.5 \mathrm{~mol} / \mathrm{L}$ tBMA solution in MeCN / water solution mixture $(\mathrm{V}: \mathrm{V}=9: 1)$ with a catalyst system of $[t \mathrm{BMA}]:[\mathrm{CuCl}]:[\mathrm{PMDETA}]=75: 1$ : 1.25 at ambient temperature. 
The DG of PtBMA increased with extension of the reaction time till 24 hours. In comparison with the SI-ATRP on PET membrane immobilized with bromide by catalyst system $\mathrm{CuCl} / \mathrm{Bpy}$ in Figure 4.12, ATRP rate is slower, because the chloride is much less reactive than bromide as discussed above. The membrane surface became more hydrophobic with contact angle $120.6^{\circ}$ (ATRP $6 \mathrm{~h}$ ). ATR/FTIR spectroscopy was used to characterize the grafted PtBMA polymer, the spectrum is shown in Fgirue 4.66.

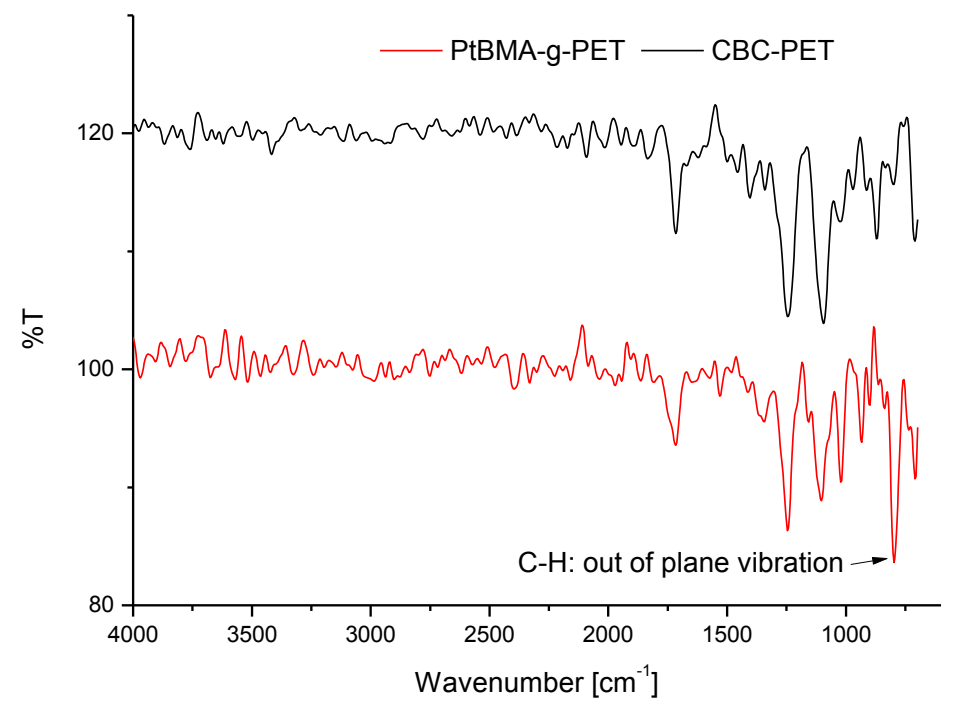

Fgirue 4.66 IR spectra of benzylchloride immobilized PET membrane and the PtBMA-g-PET membrane via $6 \mathrm{~h}$ ATRP.

After PtBMA grafting on PET membrane, only signal of $\mathrm{C}-\mathrm{H}$ (tert.-butyl group) out plane vibration was observed near wave number $775 \mathrm{~cm}^{-1}$, due to the bad back ground of with lots of ester and alkyl group.

\subsubsection{Conversion of the benzyl chloride group to dithiocarbamate group}

Iniferter DTC was immobilized on PET membrane surface through substitution of the benzyl chloride. At first, the substitution reaction was carried out in ethanol, after reaction the newly immobilized DTC was characterized via UV-initiated polymerization of AAm, but the result was not satisfactory with very low DG of PAAm. The substitution was optimized in different solvents and results are shown in Figures 4.67 and 4.68. 


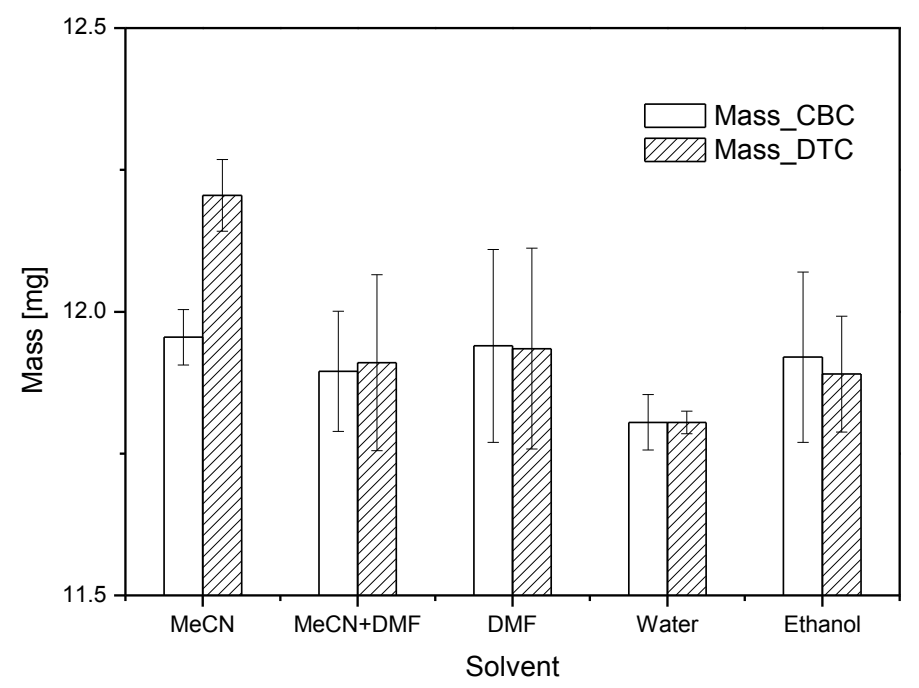

Figure 4.67 Comparison of membrane masses after benzyl chloride immobilization (CBC, open columns) and after subsequent $48 \mathrm{~h}$ DTC immobilization (DTC, solid columns), in different solvents.

Due to the limitation of DTC solubility, $0.15 \mathrm{~mol} / \mathrm{L}$ was chosen. After DTC immobilization, the membrane mass should increase. In water the membrane mass was kept the same before and after DTC immobilization. An insignificant mass reduction was found in ethanol. In DMF and mixture of DMF and MeCN (V:V=1:1), the mass of membrane had almost no change. $A$ clear increase of membrane mass was found after DTC immobilization in MeCN. The DTC group on membrane surface was identified via UV-initiated polymerization (Figure 4.68).

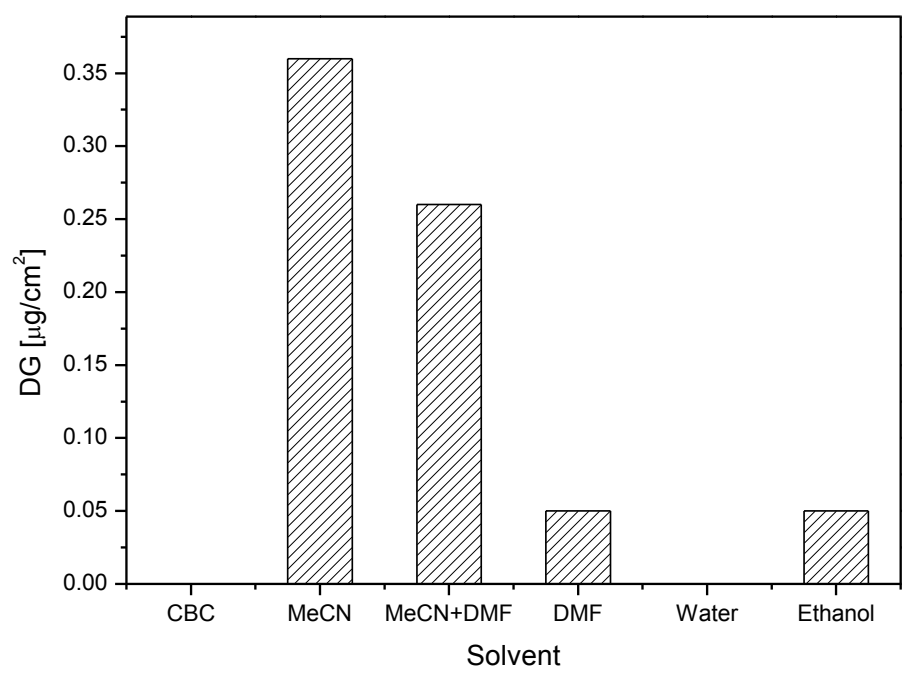


Figure 4.68 Degree of grafting for PAAm on DTC immobilized membrane in $0.3 \mathrm{~mol} / \mathrm{L}$ AAm water solution, irradiation under the UV-intensity $24 \mathrm{~mW} / \mathrm{cm}^{2}$ for $20 \mathrm{~min}$.

Before DTC immobilization, benzyl chloride had no UV-reactivity. DG of PAAm on membrane via DTC immobilization in MeCN was highest, DGs in DMF and ethanol were almost the same. DG of PAAm was increased via DTC immobilization mixture of DMA and MeCN and higher than that in pure DMF. Membrane after DTC immobilization in water had no UV-reactivity, which resulted from the interference of polar protic property of water to substitution reaction. Generally, the results of PAAm DG were in agreement with the results of mass change after DTC immobilization in different solvents (cf. Figure 4.67).

Phase transfer catalyst tert butyl ammonium bromide (TBAB) was used. The mechanism of phase transfer catalyst is shown in Figure 4.69.

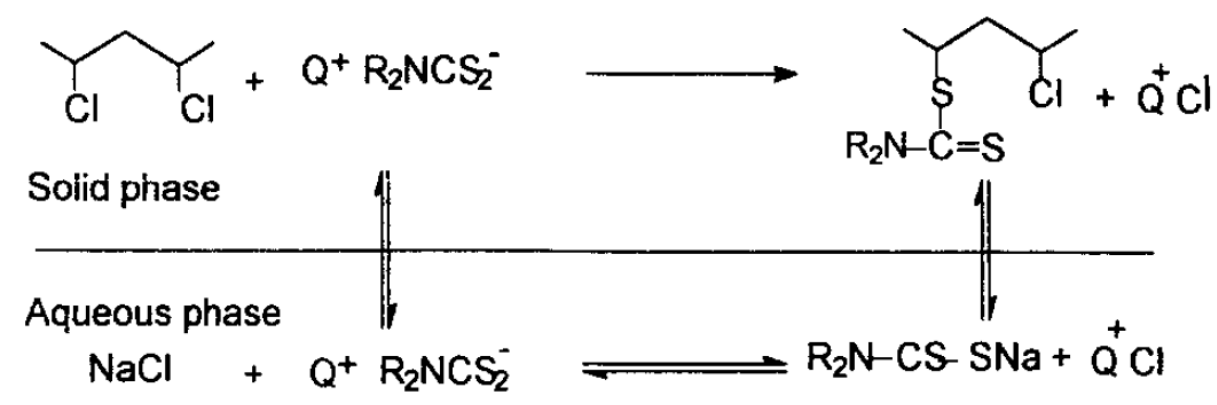

Figure 4.69 Mechanism of phase transfer catalyst to accelerate the substitution reaction. ${ }^{[169]}$

As the soluble organic tert.-butyl ammonium $\left(\mathrm{Q}^{+}\right)$transports the DTC group from the liquid phase to the organic solid phase of PET membrane, the nucleophilic substitution of chloride with DTC should take place predominantly on the surface of membrane. The reaction product chloride ion will be transported back to the organic solvent phase by the $\mathrm{Q}^{+}$and repetition of this process should accelerate the substitution reaction. ${ }^{[170]}$ After DTC immobilization with TBAB catalyst, the change of the membrane mass was measured at first and is shown in Figure 4.70. 


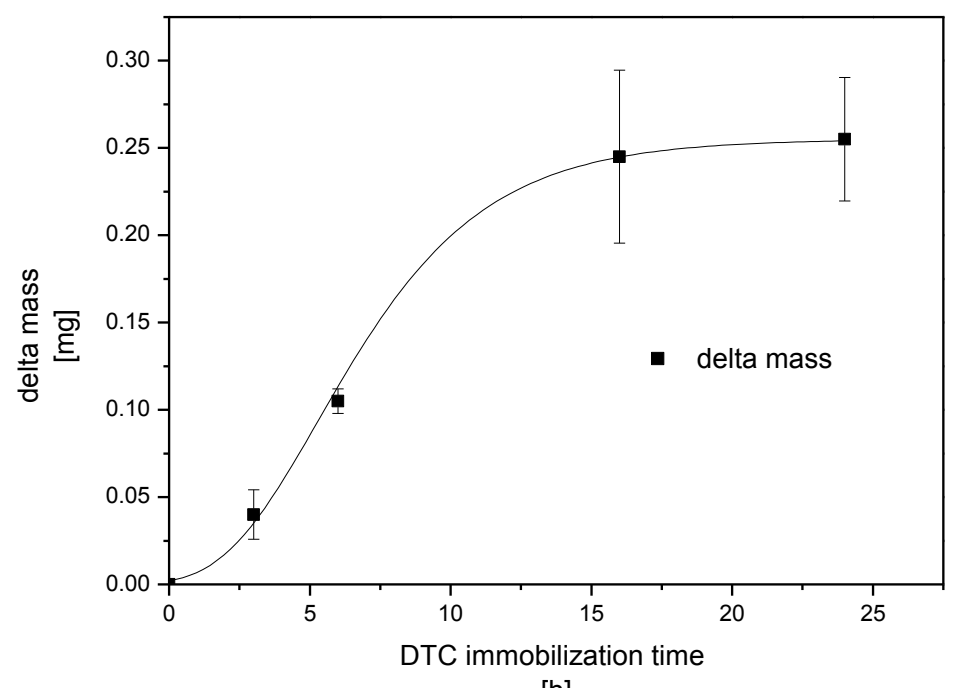

[h]

Figure 4.70 Mass increase versus the DTC immobilization time in $0.15 \mathrm{~mol} / \mathrm{L}$ DTC containing $10 \%$ TBAB (0.015 mol/L) MeCN solution.

The mass increased with the extension of the immobilization time and got saturation till $16 \mathrm{~h}$. The conversion of chloride was further confirmed via ATRP (Figure 4.71).

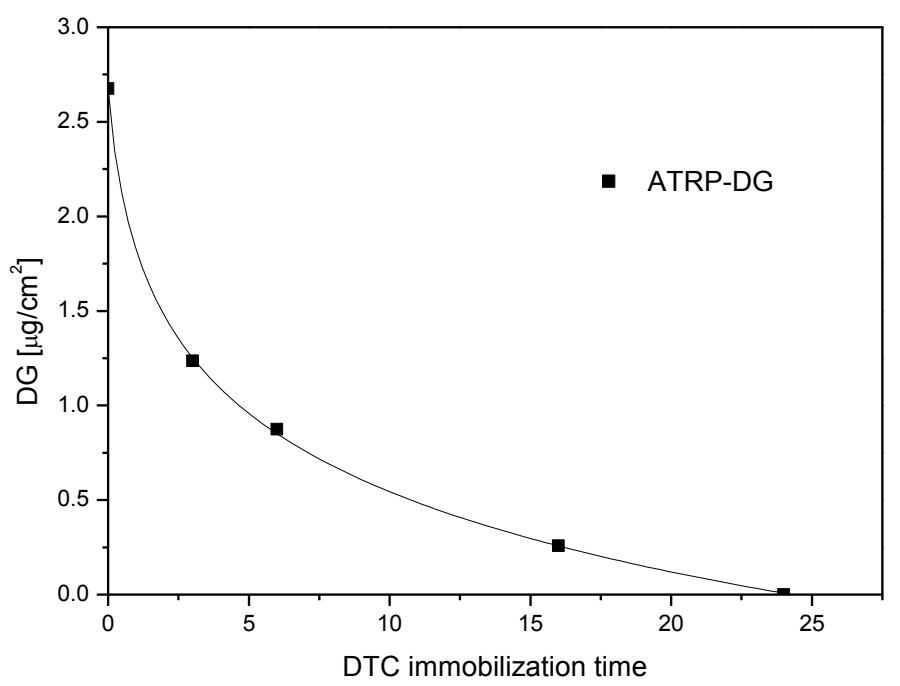

[h]

Figure 4.71 Degree of grafting for PtBMA on PET membrane surface (squares) versus DTC immobilization time, ATRP: $1.5 \mathrm{~mol} / \mathrm{L}$ tBMA solution in $\mathrm{MeCN} /$ water solution mixture $(\mathrm{V}: \mathrm{V}=$ 9:1) with a catalyst system of [tBMA]:[CUCl]:[PMDETA] $=75: 1: 1.25$ at ambient temperature for $4 \mathrm{~h}$. 
With increasing DTC immobilization time, the DG of ATRP showed a clear reducing tendency, which was corresponding to the result of mass measurement of the membrane after DTC immobilization. After $3 \mathrm{~h}$ DTC immobilization, the DG of PtBMA was about half of the CBC membrane (0 h DTC immobilization), which indicated that about half of benzyl chloride groups were converted to DTC group. After $24 \mathrm{~h}$ DTC immobilization, the sample demonstrated no reactivity of ATRP and indicated that the $C B C$ groups on the membrane surface were converted to DTC group completely. Therefore, it is feasible to control the conversion of CBC group to DTC group on the membrane surface through variation of the DTC immobilization time. After $24 \mathrm{~h}$ DTC immobilization, the DTC group was identified via XPS spectra.

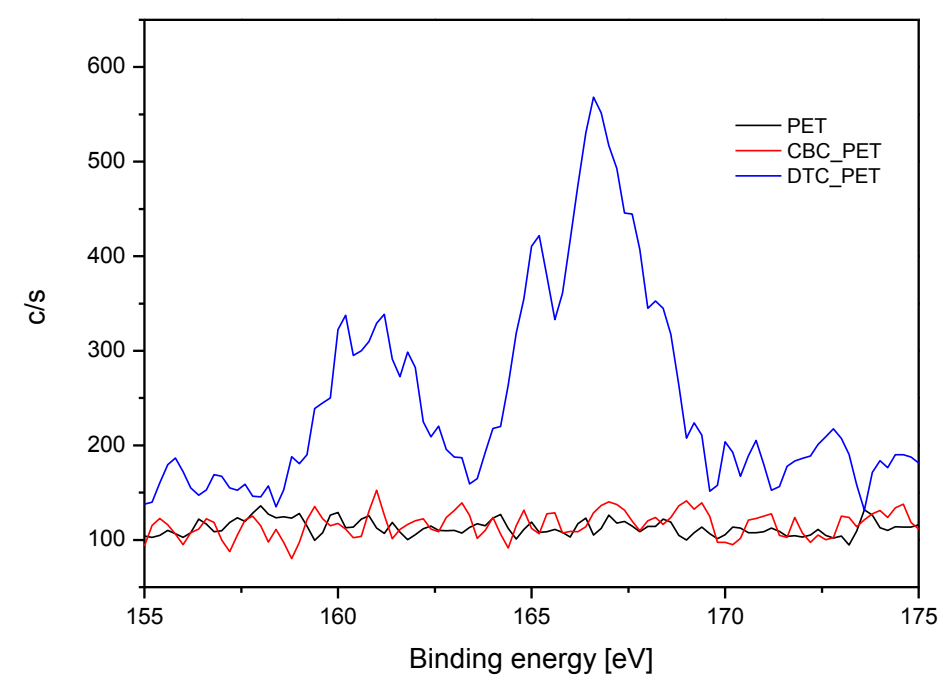

Figure 4.72 XPS spectra of the sulfur (2p) region of different membranes: black (PET): base TE PET membrane; red (CBC_PET): TE PET after benzyl chloride group immobilization; blue (DTC_PET): TE PET after DTC group immobilization for $24 \mathrm{~h}$.

Before DTC immobilization, there is no signal of sulfur from base and CBC immobilizedmembranes, the signal of S2p of DTC immobilized membrane is very clear and those data have been further confirmed by UV-polymerization. In consideration of the lower UVreactivity in $0.3 \mathrm{~mol} / \mathrm{L} \mathrm{AAm}$ aqueous solution (cf. Figure 4.68) and wetting property of the membrane, UV-polymerization of $0.7 \mathrm{~mol} / \mathrm{L} \mathrm{AAm}$ in MeCN was chosen for characterization of DTC group and result is shown in Figure 4.73. 


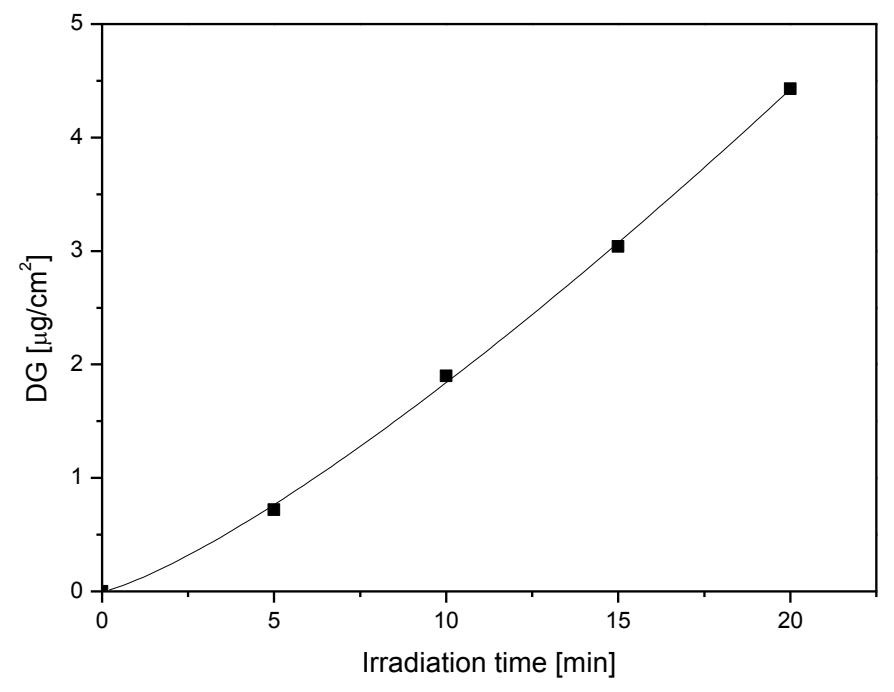

Figure 4.73 Degree of grafting for PAAm on PET membrane surface after $24 \mathrm{~h}$ DTC immobilization (squares) versus the UV-irradiation time; UV-polymerization: $0.7 \mathrm{~mol} / \mathrm{L} \mathrm{AAm}$ MeCN solution, irradiation under the UV-intensity $24 \mathrm{~mW} / \mathrm{cm}^{2}$.

Almost a linear relationship between DG of PAAm and UV-irradiation was achieved. At the beginning, the reaction rate was slow due to the low temperature of UV-box and the little content of oxygen in the UV-reaction mixtures. In comparison with the result in Figure 4.27, the control of UV-polymerization has been improved. The grafted PAAm on PET surface was further indentified via ATR/FTIR, as shown in the spectrum in Figure 4.74.

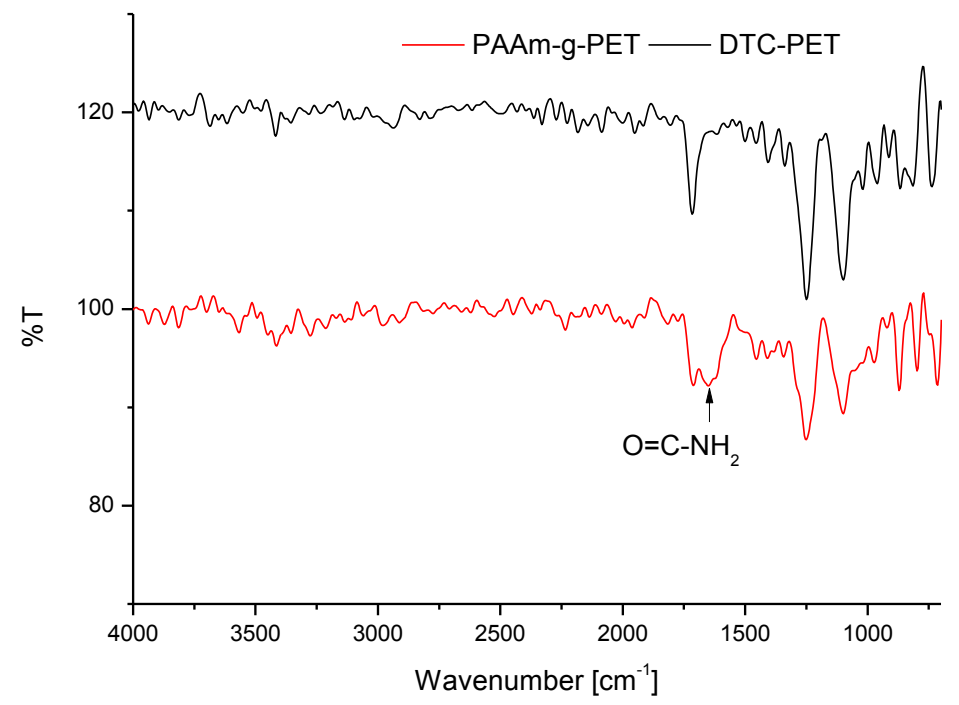


Figure 4.74 IR spectra of DTC immobilized PET membrane and the PAAm-g-PET membrane via $20 \mathrm{~min}$ UV-irradiation in $0.3 \mathrm{~mol} / \mathrm{L} \mathrm{AAm}$ water solution under the UV-intensity 24 $\mathrm{mW} / \mathrm{cm}^{2}$.

After PAAm grafting on PET membrane, the special absorption peak of amide from AAm was observed near wave number $1625 \mathrm{~cm}^{-1}$, which was not contained by the PET, benzyl and DTC structures.

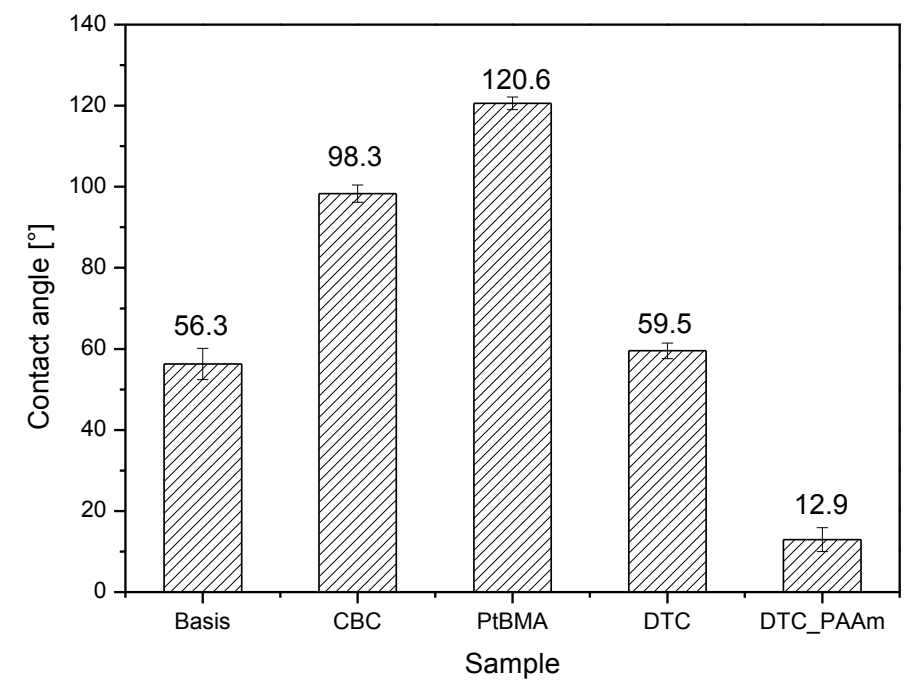

Figure 4.75 Contact angle results of membrane after different modification steps. Initiator benzyl chloride immobilization (CBC); initiator DTC immobilization; ATRP of PtBMA; UVirradiation initiated grafting of PAAm.

The contact change after benzyl chloride immobilization and its initiation have been mentioned above. After DTC immobilization, the membrane surface became more hydrophilic with the reduction of contact angle from $98.3^{\circ}$ to $59.5^{\circ}$, because DTC group with sulfur and nitrogen was able to form hydrogen bond with water. After UV-grafting of PAAm, surface became further more hydrophilic than DTC immobilized membrane with contact angle $12.9^{\circ}$, the water drop penetrated easily into the membrane. 


\subsubsection{Test of DTC stability in aqueous solution}

For the two-step grafting strategy, the scaffold was synthesized via hydrolysis in harsh acid condition (cf. section 4.4). DTC stability was first tested in the condition of hydrolysis and the result is shown in Figure 4.76.

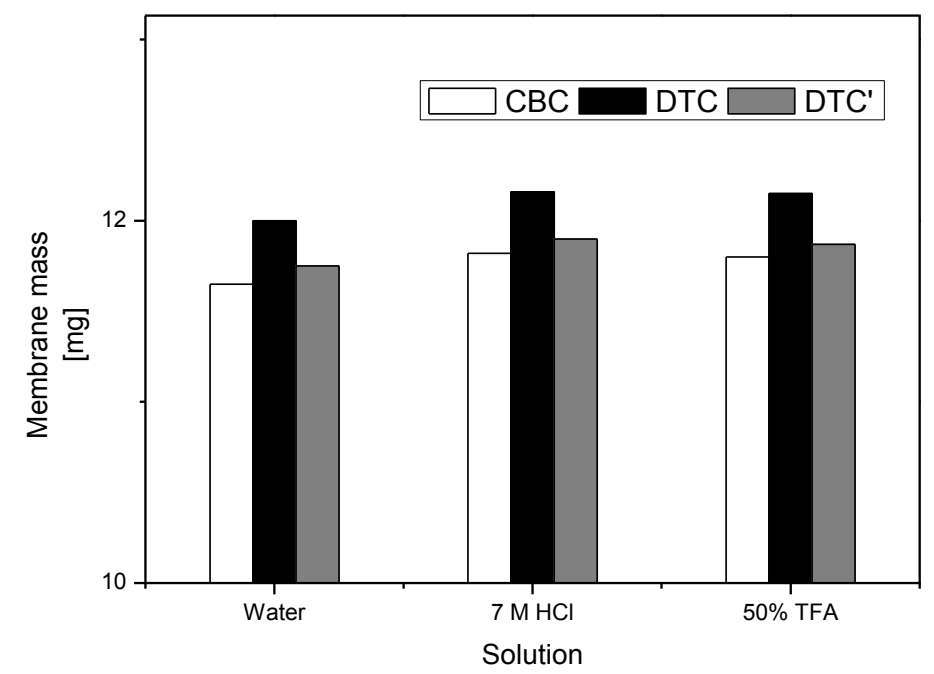

Figure 4.76 Stability of DTC immobilized membrane in hydrolysis conditions at $50{ }^{\circ} \mathrm{C}$ and for $18 \mathrm{~h}$ with shaking $200 \mathrm{rpm}$ in different solutions, white: benzyl chloride immobilized membrane; black: DTC immobilized membrane; gray: DTC immobilized membrane after immersion in hydrolysis solution.

After immersion in hydrolysis condition, the DTC membrane lost mass, because the DTC is not stable via hydrolysis of DTC group. The stability of DTC immobilized membrane was further tested in water at room temperature for $18 \mathrm{~h}$ with shaking speed $200 \mathrm{rpm}$ (Figure 4.77). 


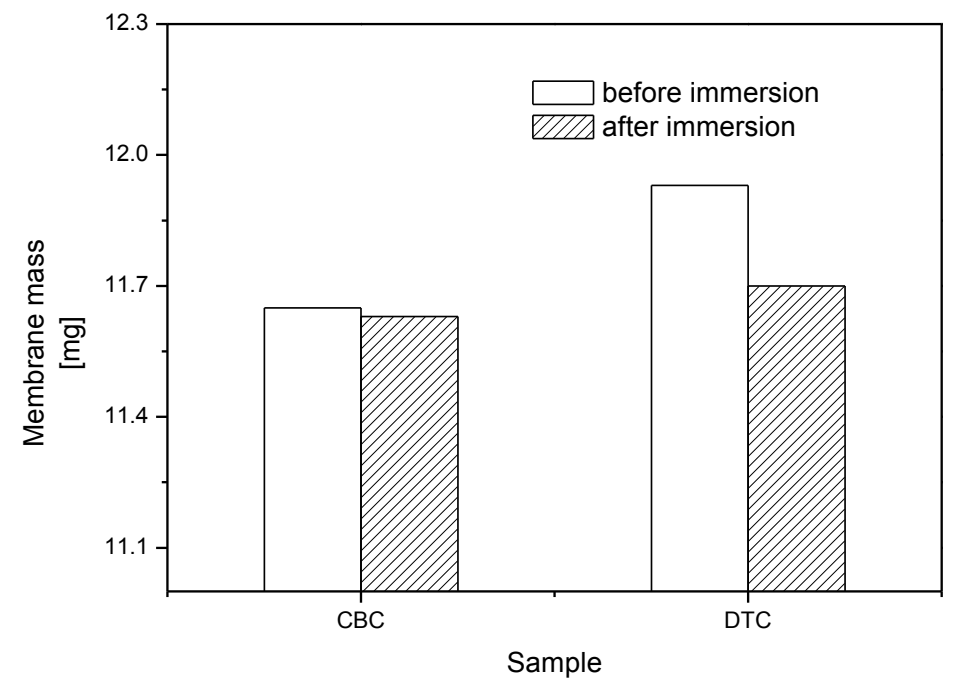

Figure 4.77 Stability of benzyl chloride and DTC immobilized membrane in water for $18 \mathrm{~h}$ at RT with shaking speed $200 \mathrm{rpm}$.

The $\mathrm{CBC}$ membrane is stable in the water, but the result of DTC membrane is similar to the stability by hydrolysis conditions. It can be concluded that the DTC immobilized membrane is not stable in aqueous solution because of hydrolysis of DTC group:
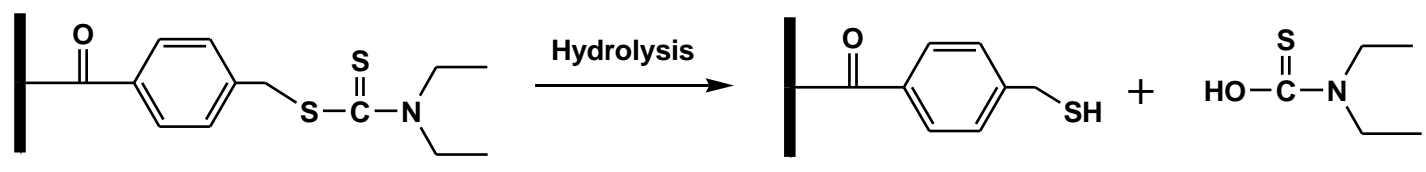

Sheme 4.4 Hydrolysis of dithiocarbamate group in aqueous solution.

Therefore, DTC can not be used for the two-step grafting strategy including the hydrolysis of PtBMA to PMAA step. Nevertheless, this initiation method combining ATRP and RAFT still shows the potential for protein imprinting such as using PDMEMA as scaffold. ${ }^{[171]}$

\subsubsection{Combination of ATRP and RAFT on PET membrane surface}

To make sure the independency of ATRP and RAFT initiations, the benzyl chloride has been proven only having ATRP reactivity and no UV-reactivity. For DTC, the non-reactivity by ATRP was proven (Figure 4.78). 


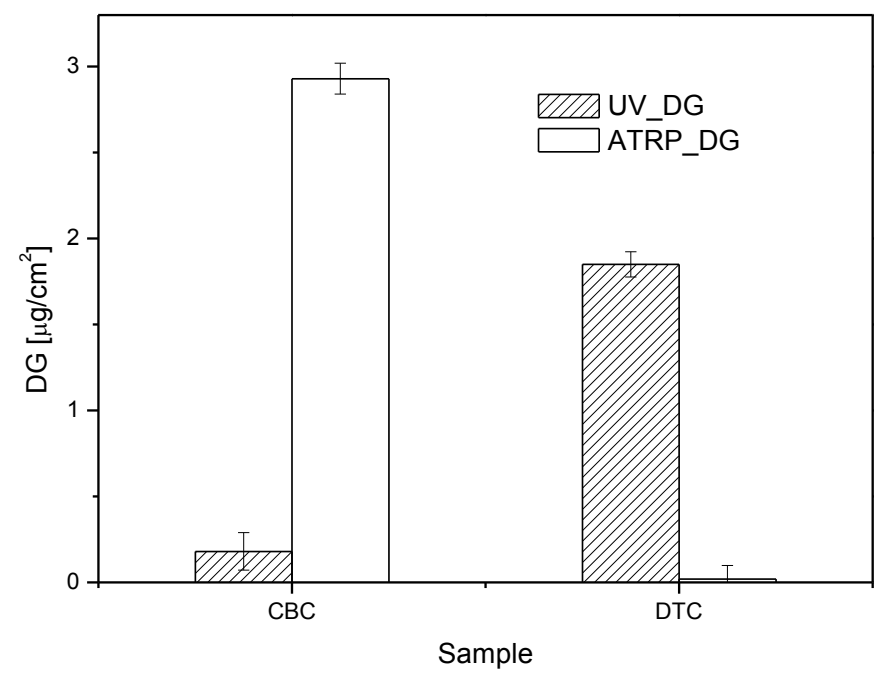

Figure 4.78 ATRP and UV-reactivity of benzyl chloride- and DTC immobilized membrane. ATRP: $1.5 \mathrm{~mol} / \mathrm{L}$ tBMA solution in solution mixture with a catalyst system of [tBMA]:[CuCl]:[PMDETA] = 75:1: 1.25 at ambient temperature for $4 \mathrm{~h}$; UV: $0.7 \mathrm{~mol} / \mathrm{L}$ AAm MeCN solution, irradiation under the UV-intensity $62 \mathrm{~mW} / \mathrm{cm}^{2}$ for $10 \mathrm{~min}$.

In order to minimize the evaporation of $\mathrm{MeCN}$ during the UV-irradiation, a short irradiation time of 10 min was chosen, but under higher UV-intensity. The slight DG of PAAm on benzyl chloride immobilized membrane should be an experimental error resulting from the weighing process or UV-activation of PET membrane. Benzyl chloride immobilized membrane had a satisfactory ATRP reactivity but no UV reactivity, while DTC group on membrane surface showed good UV-reactivity and no ATRP reactivity. The independency of reactivity by ATRP and UV-irradiation was preserved by initiation of the membrane with single initiator benzyl chloride or DTC group. The independency of benzyl chloride and DTC group by ATRP and UV-initiation on the membrane surface was characterized by variation of the DTC immobilization time (Figure 4.79). 


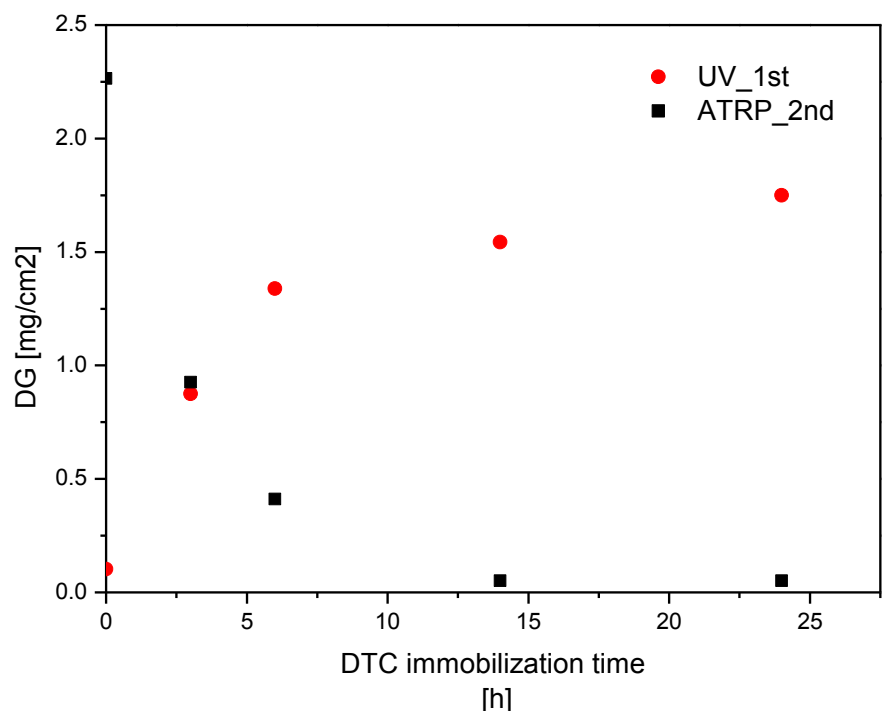

Figure 4.79 DG of PAAm (red points) via UV-initiation and DG of PTBMA (black squares) via ATRP, both versus DTC immobilization time with initiation sequence UV-initiation $\rightarrow$ ATRP; UV: $0.7 \mathrm{~mol} / \mathrm{L} \mathrm{AAm} \mathrm{MeCN}$ solution, irradiation under the UV-intensity $62 \mathrm{~mW} / \mathrm{cm}^{2}$ for $10 \mathrm{~min}$; ATRP: $1.5 \mathrm{M}$ tBMA , catalyst system [tBMA]:[CuCl]:[PMDETA] = 75:1:1.25 in MeCN for $4 \mathrm{~h}$.

The DG of PAAm increased with longer DTC immobilization time, while the DG of PtBMA by subsequent ATRP has a decreasing tendency with increasing the DTC immobilization time. This trade-off phenomenon is corresponding to the conversion of the CBC to DTC. Independency of initiation with sequence of UV-initiation $\rightarrow$ ATRP was observed. The independency was further characterized with sequence of ATRP $\rightarrow$ UV-initiation (Figure 4.80).

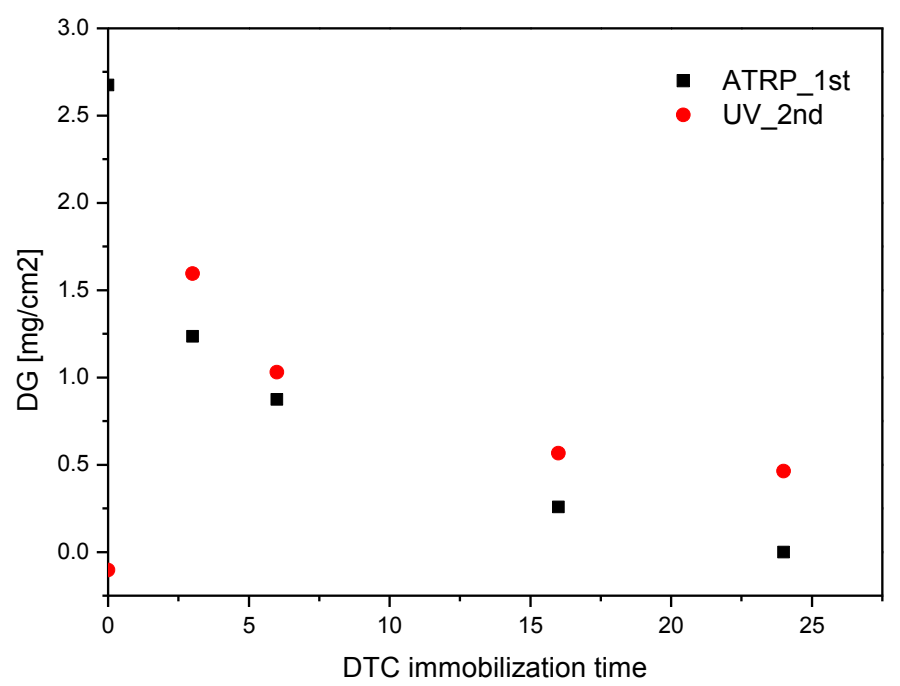

[h] 
Figure 4.80 DG of PAAm (red points) via UV-initiation and DG of PTBMA(black squares) via ATRP versus DTC immobilization time with initiation sequence ATRP $\rightarrow$ UV-initiation; UV: UV: $0.7 \mathrm{~mol} / \mathrm{L} \mathrm{AAm} \mathrm{MeCN}$ solution, irradiation under the UV-intensity $62 \mathrm{~mW} / \mathrm{cm}^{2}$ for $15 \mathrm{~min}$; ATRP: $1.5 \mathrm{M}$ tBMA, catalyst system [tBMA]:[CuCl]:[PMDETA] = 75:1:1.25 in MeCN for $4 \mathrm{~h}$.

DG of PtBMA by ATRP has a decreasing tendency with increase of the DTC immobilization time, which was corresponded to the results when using the reverse sequence. However,the values are slightly higher, as schown in Figure 4.79, which resulted from the steric effect of grafted PAAm prepared via UV-irradiation. In order to overcome the steric effect of PtBMA, the UV-irradiation time was extended to $15 \mathrm{~min}$ to ensure enough PAAm grafting for characterization. The DG of PAAm was much different to the result in Figure 4.79. DG of PAAm increased from 0 to $3 \mathrm{~h}$ DTC immobilization time, then decreased until $24 \mathrm{~h}$ immobilization time. The interesting thing is, the samples via more than $3 \mathrm{~h}$ DTC immobilization time had yellow color on membrane surface after ATRP, that has already been noted in experiments shown in Figure 4.71, but only ATRP had been tested in that experiment. The complex of the $\mathrm{N}$ atom (DTC) and the copper I or II was the reason, ${ }^{[172]}$ the DTC group lost the UV-reactivity after complexation. ${ }^{[173]}$ The ability of DTC to form complex with copper I and II is dependent on the density of DTC, when DTC group has less than $50 \%$ surface coverage (according to the degree of grafting of ATRP and UV-polymerization in Figure 4.80), the distance between two DTC group is too far to form the complex (Figure 4.81). The poor solubility of EDTA in organic solution and the poor stability of DTC in aqueous solution are the main obstacles to remove the copper from the membrane surface.

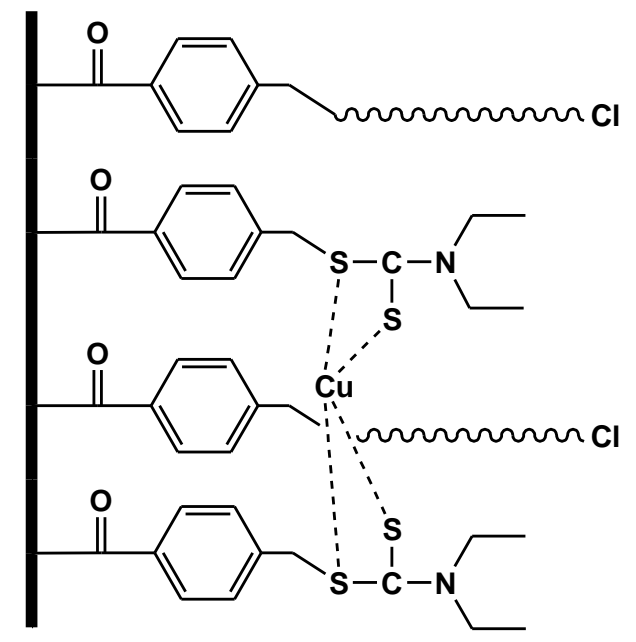

Figure 4.81 Complex of DTC and copper formed on the membrane surface. 
The independency of the two initiations can only preserved on the surface covered by no more than 50\% DTC group or via DTC immobilization time not longer than $3 \mathrm{~h}$. This irradiation model still has strong potential for protein imprinting with both precise scaffold grafting and precise subsequent crosslinking. 


\section{Chapter 5 Conclusions and outlook}

New methods for two-step surface grafting in combination with molecular imprinting for proteins had been investigated and developed.

ATRP initiator isobutyl bromide density on TE PET membrane surface was improved about 2.6 times via extension of the activation time from $3 \mathrm{~h}$ to $6 \mathrm{~h}$ during coupling the linker for the bromide (cf. Scheme 1.1). The conversions of the surface groups were characterized through reversible ionic dye binding of carboxyl group and XPS spectra of nitrogen and bromide region. Surface grafting of PtBMA on TE PET membrane immobilized with bromide was controlled very precisely in $1.5 \mathrm{~mol} / \mathrm{L} t \mathrm{BMA} \mathrm{MeCN} /$ water $(\mathrm{V}: \mathrm{V}=9: 1)$ solution with a catalyst system of $[t \mathrm{BMA}]:[\mathrm{CuCl}]:\left[\mathrm{CuCl}_{2}\right]:[\mathrm{Bpy}]=75: 1: 0.1: 2.75$ at ambient temperature. Ligand variation, solvent variation, solvent mixture with water, addition of $\mathrm{Cu}(\mathrm{II})$, reduction of $t B M A$ concentration, and improvement of Bpy ratio were investigated to optimize the performance of SI-ATRP. Hydrolysis of PtBMA to PMAA was optimized and the condition of $50 \%$ TFA solution in $\mathrm{MeCN} /$ water mixture $(\mathrm{V}: \mathrm{V}=1: 1)$ at $50{ }^{\circ} \mathrm{C}$ for $18 \mathrm{~h}$ was chosen. The surface grafting was also characterized by measuring the change of pore size via permporometry (dry state) and hydrodynamic layer thickness (wet state) and contact angle. Conformation of polyelectrolyte PMAA was investigated via hydrodynamic layer thickness; the response of layer thickness to $\mathrm{pH}$, ionic strength and polarity was successfully characterized.

After PtBMA hydrolysis, the UV-reactivity of carbon-bromide sites on TE PET and cellulose membrane surface was investigated in aqueous solution containing AAm and MBAA via variations of the UV-intensity and monomer concentration, in order to obtain a similar DG range of crosslinked PAAm relative to that of PMAA (via 0 to $4 \mathrm{~h}$ ATRP of tBMA and subsequent hydrolysis). $0.3 \mathrm{~mol} / \mathrm{L} \mathrm{AAm}$ and MBAA (crosslinking degree $=12 \%$ ) in aqueous solution under the UV-intensity of about $24 \mathrm{~mW} / \mathrm{cm}^{2}$ was chosen for Lyz and IgG imprinting on TE PET membrane.

The novel protein imprinting method toward preparation of materials suited for selective separation of Lyz and CyC has been successfully developed via two-step grafting on PET membrane surface by combination of SI-ATRP and UV-initiated grafting/crosslinking copolymerization. "Orthogonal" initiation of the carbon-bromide initiator site for both SI- 
ATRP and UV-grafting/crosslinking had been proven feasible. The respective optimizations by focusing onto the effects by the individual steps $-1^{\text {st }}$ step: scaffold length via variation the ATRP time from $1 \mathrm{~h}$ to $4 \mathrm{~h}$ and subsequent hydrolysis; $2^{\text {nd }}$ step: variations through UV time from 10 to $25 \mathrm{~min}$ and degree of crosslinking from $10 \%$ to $15 \%$ - have significantly improved the property of the MIP membrane adsorber. A tradeoff between protein binding capacity and selectivity (up to 4.5 when measured with single protein solutions) had been observed. Shorter scaffold chains from $1^{\text {st }}$ step and longer UV time in the $2^{\text {nd }}$ step lead to higher selectivity but lower capacity. The degree of crosslinking could be used to optimize the materials with respect to both targets. The Lyz vs. CyC selectivity of the MIP had reached about 18 when assessed by a 1:1 mixture of the two proteins. The imprinting effect was demonstrated by comparison of the protein binding capacity and selectivity of MIP and NIP. Via measuring adsorption isotherm, the MIP has been shown to have much higher affinity to Lyz with $\mathrm{K}_{\mathrm{L}}=599 \mathrm{~mL} / \mathrm{mg}$ than that of NIP with $\mathrm{K}_{\mathrm{L}}=216 \mathrm{~mL} / \mathrm{mg}$. The heterogeneity of imprinted structures was observed by adsorption of Lyz with varied salt concentrations. Addition of functional monomers such as DMAEMA during UV-grafting/crosslinking improved the recognition property of imprinted membrane with a high selectivity of 6.5 (measured with single protein).

Two-step grafting strategy was then evaluated by imprinting of IgG with bigger molar mass than Lyz on TE PET membrane, through variation of scaffold length (DG) from 0.55 to 0.97 $\mu \mathrm{g} / \mathrm{cm}^{2}$ and crosslinking degree from $10 \%$ to $12 \%$. Due to the big size of IgG, the poor mass transport was observed by comparison of the eluted IgG content directly after UVgrafting/crosslinking and the rebinding IgG. The optimized performance of IgG imprinting was obtained with IgG binding capacity of $5.8 \mathrm{mg} / \mathrm{mL}$ and selectivity of IgG vs. HSA of $~ 5$ (measured with single protein).

The results of Lyz and IgG imprinting on TE PET membrane are summarized in Figure 5.1. 


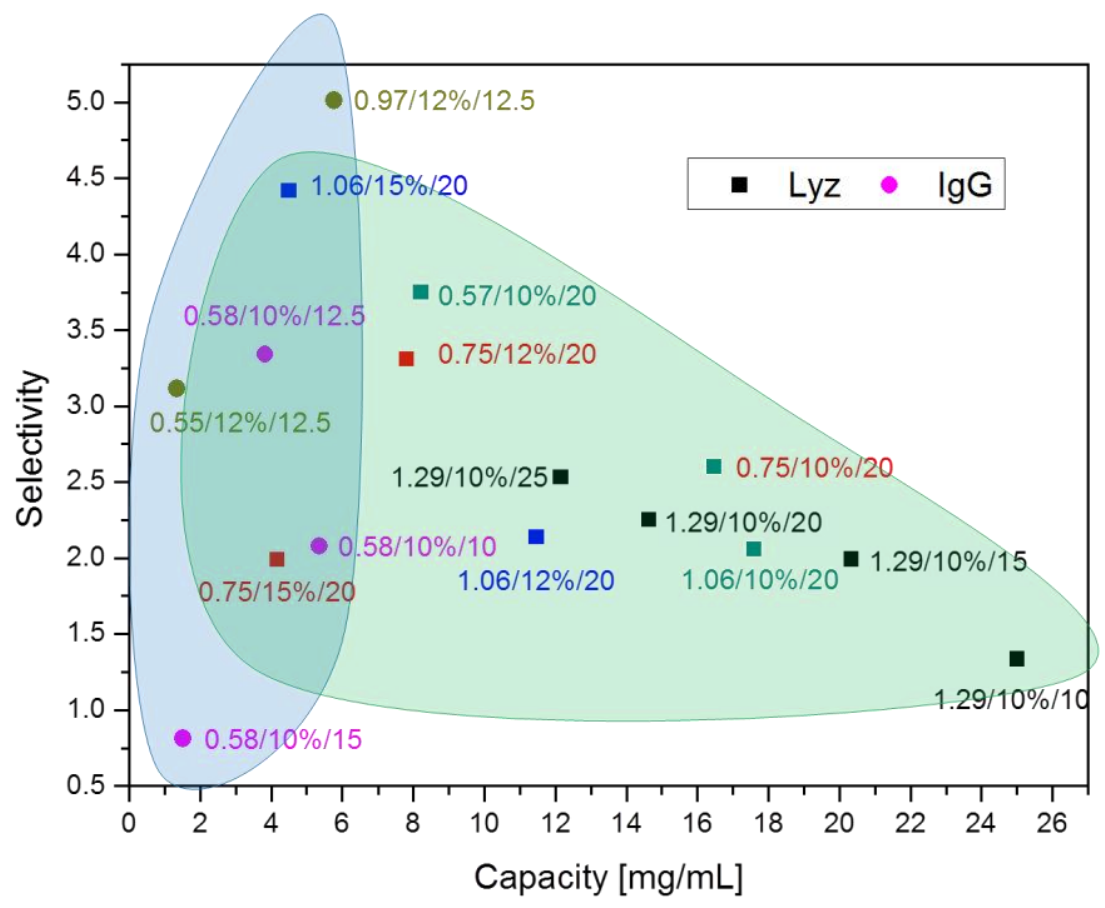

Figure 5.1 Summary of Lyz and IgG imprinting on TE PET membranes (selectivity from single protein adsorption). Squares represent the Lyz imprinting (green area) and points represent the IgG imprinting (blue area), legends represent the imprinting conditions in form of DG [min]/crosslinking degree/UV-irradiation time [min], the same color means the same series.

A trade-off between protein binding capacity and selectivity had been observed for both Lyz imprinting and IgG imprinting. Long scaffold chain led a higher protein binding capacity but lower selectivity of imprinted membrane. Improvements of UV-irradiation time and crosslinking degree led to a higher selectivity but lower capacity. Due to the big size of IgG, most points corresponding to results for IgG imprinting are closer to the coordinate origin than the ones for Lyz, indicating a lower overall performance for IgG than Lyz imprinting under similar condition. However, this was mainly because of lower lgG binding capacities.

Imprinting of Lyz via two-step grafting was also achieved on irregular porous cellulose membrane with much higher surface area than TE PET membrane. Surface grafting in 1.5 $\mathrm{mol} / \mathrm{L}$ tBMA MeCN / water mixture solution ( $\mathrm{V}: \mathrm{V}=9: 1)$ with a catalyst system of $[t \mathrm{BMA}]:[\mathrm{CuCl}]:\left[\mathrm{CuCl}_{2}\right]:[\mathrm{Bpy}]=75: 1: 0.1: 2.75$ at ambient temperature was transferred successfully to the cellulose membrane. Hydrolysis was carried out in a less strong acid solution $35 \%$ TFA solution in MeCN / water mixture $(\mathrm{V}: \mathrm{V}=1: 1)$ at $50{ }^{\circ} \mathrm{C}$ for $14 \mathrm{~h}$, because 
cellulose has less stability in acid solution than PET. Due to the high porosity and tortuous morphology of cellulose membrane, high UV-intensity $\left(\sim 7 \mathrm{~mW} / \mathrm{cm}^{2}\right)$ and monomer concentration of $0.5 \mathrm{~mol} / \mathrm{L}$ AAm and MBAA was applied to obtained satisfactory property of imprinted membrane. The property of imprinted structure was improved via variation of UVirradiation time, UV-intensity, monomer concentration during UV-polymerization, crosslinking degree and scaffold length. Heat produced by the high UV-intensity played more important role than imprinting on PET membrane under lower UV-intensity. Heat led to reduction of the recognition property but higher binding capacity. The Lyz vs. CyC selectivity of the MIP had reached about 14 when assessed by in a 1:1 mixture of the two proteins. The imprinting effect was demonstrated by comparison of the protein binding capacity and selectivity of MIP and NIP, the MIP has much higher affinity to Lyz with $\mathrm{K}_{\mathrm{L}}=259 \mathrm{~mL} / \mathrm{mg}$ than that of NIP with $\mathrm{K}_{\mathrm{L}}=76 \mathrm{~mL} / \mathrm{mg}$ via measuring isotherm adsorption, although the binding capacity of NIP was higher than that of MIP.

The comparison of Lyz imprinting on PET and cellulose membrane is shown in Figure 5.2. In order to better compare the two substrates, the capacity was calculated in terms of bound protein mass relative to specific surface area.

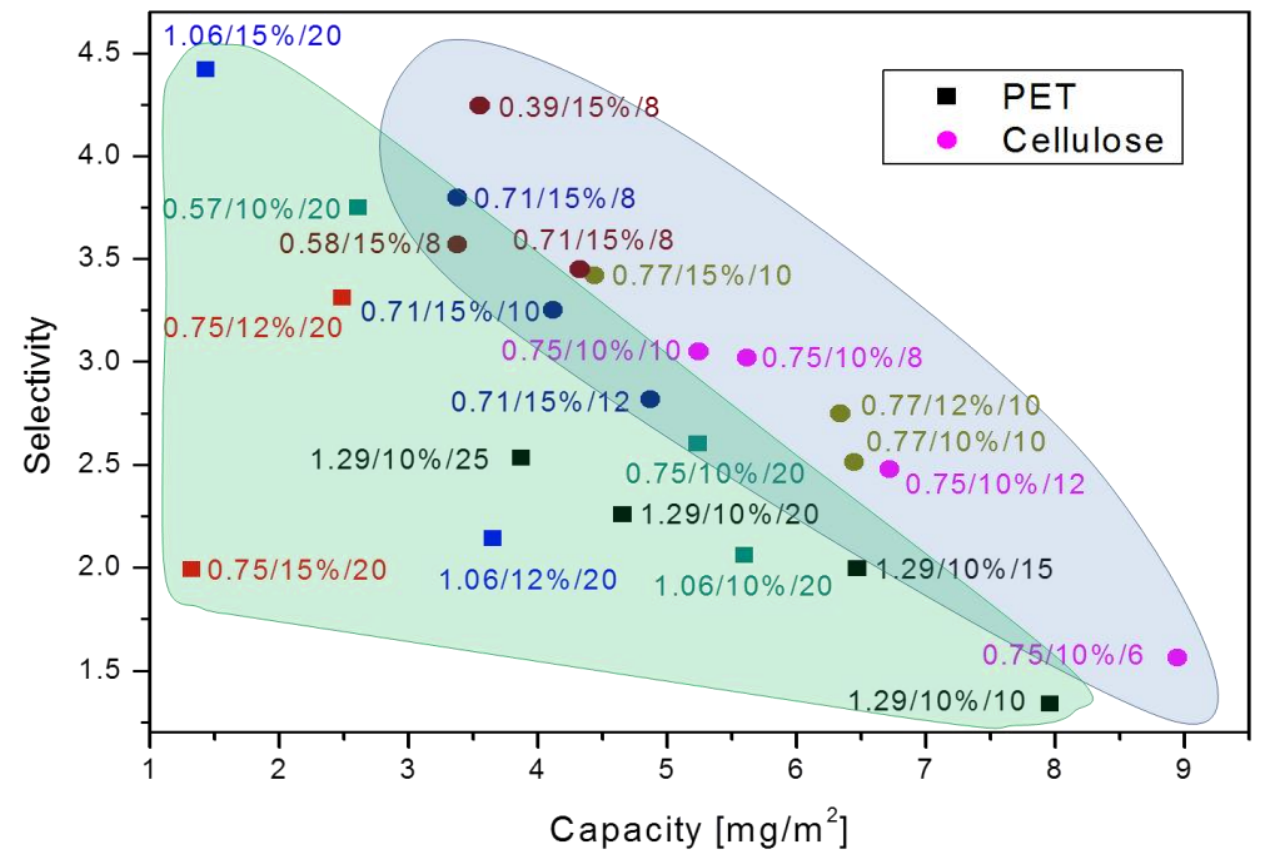

Figure 5.2 Comparison of Lyz imprinting on TE PET membrane (squares, green area) and cellulose membrane (points, blue area) using selectivity from single protein adsorption and 
binding capacity values normalized to specific surface area; legends represent the imprinting condition in form of DG [min]/crosslinking degree/UV-irradiation time [min], the same color means the same series.

The trade-off phenomenon between protein binding capacity and selectivity has been also found by Lyz imprinting on cellulose membrane. The Lyz imprinting on cellulose has better performance than that on PET membrane, because most data points are located farther from the coordinate origin than the squares for imprinting on PET membrane.

In order to improve the control of UV-grafting/crosslinking for protein imprinting, combination of ATRP and RAFT was developed on the TE PET membrane surface via immobilization of benzyl chloride and dithiocarbamate (DTC) group, which can be initiated independently. First, the benzyl chloride was successfully immobilized and characterized via ATRP in $1.5 \mathrm{~mol} / \mathrm{L}$ tBMA MeCN / water $(\mathrm{V}: \mathrm{V}=9: 1)$ solution with a catalyst system of $[$ tBMA]:[CuCl]:[PMDETA] $=75: 1: 1.25$ at ambient temperature. DTC immobilization was achieved via the subsequent substitution of chloride and catalyzed by phase transfer agent TABA. Manipulation of the chloride to DTC group conversion was achieved via variation of the DTC immobilization time. For $3 \mathrm{~h}$, the conversion reached $50 \%$ and got $100 \%$ till $24 \mathrm{~h}$. DTC initiation was well controlled in $0.7 \mathrm{~mol} / \mathrm{LAAm} \mathrm{MeCN}$ solution, irradiation under the UV-intensity $24 \mathrm{~mW} / \mathrm{cm}^{2}$ with linear relationship between $D_{A A m}$ and irradiation time. DTC group has a poor stability in aqueous solution, which limited the application by the strategy of the scaffold PMAA synthesized via hydrolysis of PtBMA. Independency irradiation of benzyl chloride and DTC was investigated by two initiation sequences: UV-initiation $\rightarrow$ ATRP and ATRP $\rightarrow$ UV-initiation with different ratio of DTC and benzyl chloride group. Independency of initiation via ATRP and UV-polymerization was observed when the conversion of benzyl chloride to DTC was not more than $50 \%$. The complexation of DTC and copper depends on the density of DTC on the membrane surface. After complexation, the UV-reactivity of DTC was reduced significantly.

The technique presented in this work is general and highly versatile through combining the SI-ATRP and the robust molecular imprinting techniques using standard commercial monomers and initiator. The approach has a large potential to be applied for preparing materials for downstream processing, in particular protein capturing. It can be expected that imprinting specificity and target affinity can significantly be improved by using more refined 
multifunctional scaffolds which are complementary to protein surface such as adjusted functional group density and specific combinations of different functionalities. Based on established surface imprinting and scaffold imprinting, integration of the epitope imprinting strategy is very promising to minimize the obstacles resulting from the big protein size and its flexible conformation. Furthermore, crosslinking via living radical polymerization such as ATRP, RAFT, and NMP with precise control on the polymer growth provide a huge potential to further improve the property of imprinted structure. 


\section{References}

[1] G. Wulff, Molecular Imprinting in Cross-Linked Materials with the Aid of Molecular Templates - a Way Towards Artificial Antibodies, Angew Chem Int Edit 1995, 34, 1812-1832.

[2] K. Mosbach, O. Ramstrom, The emerging technique of molecular imprinting and its future impact on biotechnology, Bio-Technol 1996, 14, 163-170.

[3] C. Alexander, H. S. Andersson, L. I. Andersson, R. J. Ansell, N. Kirsch, I. A. Nicholls, J. O'Mahony, M. J. Whitcombe, Molecular imprinting science and technology: a survey of the literature for the years up to and including 2003, J Mol Recognit 2006, 19, 106-180.

[4] N. W. Turner, C. W. Jeans, K. R. Brain, C. J. Allender, V. Hlady, D. W. Britt, From 3D to 2D: A review of the molecular imprinting of proteins, Biotechnol Progr 2006, 22, 1474-1489.

[5] A. Bossi, F. Bonini, A. P. F. Turner, S. A. Piletsky, Molecularly imprinted polymers for the recognition of proteins: The state of the art, Bioelectron 2007, 22, 1131-1137.

[6] D. R. Kryscio, N. A. Peppas, Critical review and perspective of macromolecularly imprinted polymers, Acta Biomater 2012, 8, 461-473.

[7] T. Kobayashi, Scaffold Impring, in M. Yang and O. Ramström, Molecular Imprinted Materials, Marcle Dekker, New York, 2005, pp. 285-306.

[8] Y. Long, X. C. Xing, R. F. Han, Y. Sun, Y. Wang, Z. Zhao, H. F. Mi, Two-step purification of lowcontent cellular protein using protein-imprinted polymers, Anal Biochem 2008, 380, 268-275.

[9] Y. Hoshino, H. Koide, T. Urakami, H. Kanazawa, T. Kodama, N. Oku, K. J. Shea, Recognition, Neutralization, and Clearance of Target Peptides in the Bloodstream of Living Mice by Molecularly Imprinted Polymer Nanoparticles: A Plastic Antibody, J Am Chem Soc 2010, 132, 6644-6645.

[10] C. Geismann, A. Yaroshchuk, M. Ulbricht, Permeability and electrokinetic characterization of poly(ethylene terephthalate) capillary pore membranes with grafted temperature-responsive polymers, Langmuir 2007, 23, 76-83.

[11] A. Friebe, M. Ulbricht, Controlled pore functionalization of poly(ethylene terephthalate) track-etched membranes via surface-initiated atom transfer radical polymerization, Langmuir 2007, 23, 10316-10322.

[12] D. M. He, W. Sun, T. Schrader, M. Ulbricht, Protein adsorbers from surface-grafted copolymers with selective binding sites, J Mater Chem 2009, 19, 253-260.

[13] K. Matyjaszewski, N. V. Tsarevsky, Nanostructured functional materials prepared by atom transfer radical polymerization, Nat Chem 2009, 1, 276-288.

[14] F. Tomicki, D. Krix, H. Nienhaus, M. Ulbricht, Stimuli-responsive track-etched membranes via surface-initiated controlled radical polymerization: Influence of grafting density and pore size, J Membrane Sci 2011, 377, 124-133.

[15] M. Ulbricht, A. Oechel, Photo-bromination and photo-induced graft polymerization as a twostep approach for surface modification of polyacrylonitrile ultrafiltration membranes, Eur Polym J 1996, 32, 1045-1054. 
[16] G. Wulff, G. Kirstein, Measuring the Optical-Activity of Chiral Imprints in Insoluble Highly Cross-Linked Polymers, Angew Chem Int Edit 1990, 29, 684-686.

[17] B. N. Chen, S. Piletsky, A. P. F. Turner, Molecular recognition: Design of "keys", Comb Chem High T Scr 2002, 5, 409-427.

[18] K. Haupt, K. Mosbach, Molecularly imprinted polymers and their use in biomimetic sensors, Chem Rev 2000, 100, 2495-2504.

[19] K. Mosbach, R. Mosbach, Entrapment of Enzymes and Microorganisms in Synthetic CrossLinked Polymers and Their Application in Column Techniques, Acta Chem Scand 1966, 20, 2807-2810.

[20] G. Wulff, A. Sarhan, The Use of Polymers with Enzyme-Analogous Structures for Resolution of Racemates, Angew Chem Int Edit 1972, 11, 341-341.

[21] http://www.nascent.qmul.ac.uk/.

[22] D. L. Nelson and M. M. Cox, Lehninger principles of biochemist, Wiley-VCH, New York, 2004.

[23] Http://academic.brooklyn.cuny.edu/biology/bio4fv/page/3d_prot.htm.

[24] T. Ando, Fundamentals of protein structure (ppt), Tokyo University of Science, 2006.

[25] J. L. Liao, Y. Wang, S. Hjerten, Novel support with artificially created recognition for the selective removal of proteins and for affinity chromatography, Chromatographia 1996, 42, 259-262.

[26] S. Hjerten, J. L. Liao, K. Nakazato, Y. Wang, G. Zamaratskaia, H. X. Zhang, Gels mimicking antibodies in their selective recognition of proteins, Chromatographia 1997, 44, 227-234.

[27] D. Tong, C. Hetenyi, Z. Bikadi, J. P. Gao, S. Hjerten, Some studies of the chromatographic properties of gels ('artificial antibodies/receptors') for selective adsorption of proteins, Chromatographia 2001, 54, 7-14.

[28] X. S. Pang, G. X. Cheng, S. L. Lu, E. J. Tang, Synthesis of polyacrylamide gel beads with electrostatic functional groups for the molecular imprinting of bovine serum albumin, Anal Bioanal Chem 2006, 384, 225-230.

[29] J. T. Huang, J. Zhang, J. Q. Zhang, S. H. Zheng, Template imprinting amphoteric polymer for the recognition of proteins, J Appl Polym Sci 2005, 95, 358-361.

[30] S. H. Ou, M. C. Wu, T. C. Chou, C. C. Liu, Polyacrylamide gels with electrostatic functional groups for the molecular imprinting of lysozyme, Anal Chim Acta 2004, 504, 163-166.

[31] H. Q. Shi, W. B. Tsai, M. D. Garrison, S. Ferrari, B. D. Ratner, Template-imprinted nanostructured surfaces for protein recognition, Nature 1999, 398, 593-597.

[32] X. B. Hu, G. T. Li, J. Huang, D. Zhang, Y. Qiu, Construction of self-reporting specific chemical sensors with high sensitivity, Adv Mater 2007, 19, 4327-4334.

[33] L. Qin, X. W. He, W. Zhang, W. Y. Li, Y. K. Zhang, Surface-modified polystyrene beads as photografting imprinted polymer matrix for chromatographic separation of proteins, $J$ Chromatogr A 2009, 1216, 807-814. 
[34] Q. Q. Gai, F. Qu, Z. J. Liu, R. J. Dai, Y. K. Zhang, Superparamagnetic lysozyme surfaceimprinted polymer prepared by atom transfer radical polymerization and its application for protein separation, J Chromatogr A 2010, 1217, 5035-5042.

[35] A. Rachkov, N. Minoura, Recognition of oxytocin and oxytocin-related peptides in aqueous media using a molecularly imprinted polymer synthesized by the epitope approach, $J$ Chromatogr A 2000, 889, 111-118.

[36] H. Nishino, C. S. Huang, K. J. Shea, Selective protein capture by epitope imprinting, Angew Chem Int Edit 2006, 45, 2392-2396.

[37] J. L. Urraca, M. A. C. Moreno-Bondi, A. J. Hall, B. Sellergren, Direct extraction of penicillin g and derivatives from aqueous samples using a stoichiometrically imprinted polymer, Anal Chem 2007, 79, 695-701.

[38] O. Vigneau, C. Pinel, M. Lemaire, lonic imprinted resins based on EDTA and DTPA derivatives for lanthanides(III) separation, Anal Chim Acta 2001, 435, 75-82.

[39] H. Asanuma, T. Akiyama, K. Kajiya, T. Hishiya, M. Komiyama, Molecular imprinting of cyclodextrin in water for the recognition of nanometer-scaled guests, Anal Chim Acta 2001, 435, 25-33.

[40] W. J. Wizeman, P. Kofinas, Molecularly imprinted polymer hydrogels displaying isomerically resolved glucose binding, Biomaterials 2001, 22, 1485-1491.

[41] P. S. Reddy, T. Kobayashi, M. Abe, N. Fujii, Molecular imprinted Nylon-6 as a recognition material of amino acids, Eur Polym J 2002, 38, 521-529.

[42] M. J. Guo, Z. Zhao, Y. G. Fan, C. H. Wang, L. Q. Shi, J. J. Xia, Y. Long, H. F. Mi, Protein-imprinted polymer with immobilized assistant recognition polymer chains, Biomaterials 2006, 27, 43814387.

[43] M. Szwarc, Living Polymers, Nature 1956, 178, 1168-1169.

[44] W. A. Braunecker, K. Matyjaszewski, Controlled/living radical polymerization: Features, developments, and perspectives, Prog Polym Sci 2007, 32, 93-146.

[45] A. H. E. Müller and K. Matyjaszewski, Controlled and Living Polymerizations, Wiley-VCH, Weinheim, 2009.

[46] C. J. Hawker, A. W. Bosman, E. Harth, New polymer synthesis by nitroxide mediated living radical polymerizations, Chem Rev 2001, 101, 3661-3688.

[47] M. K. Georges, R. P. N. Veregin, P. M. Kazmaier, G. K. Hamer, Narrow Molecular-Weight Resins by a Free-Radical Polymerization Process, Macromolecules 1993, 26, 2987-2988.

[48] D. Bertin, F. Chauvin, S. Marque, P. Tordo, Lack of chain length effect on the rate of homolysis of polystyryl-SG1 alkoxyamines, Macromolecules 2002, 35, 3790-3791.

[49] D. Benoit, V. Chaplinski, R. Braslau, C. J. Hawker, Development of a universal alkoxyamine for "living" free radical polymerizations, J Am Chem Soc 1999, 121, 3904-3920. 
[50] A. Studer, K. Harms, C. Knoop, C. Muller, T. Schulte, New sterically hindered nitroxides for the living free radical polymerization: X-ray structure of an alpha-H-bearing nitroxide, Macromolecules 2004, 37, 27-34.

[51] G. Moad, E. Rizzardo, S. H. Thang, Living radical polymerization by the RAFT process, Aust J Chem 2005, 58, 379-410.

[52] Y. K. Chong, J. Krstina, T. P. T. Le, G. Moad, A. Postma, E. Rizzardo, S. H. Thang, Thiocarbonylthio compounds $[\mathrm{S}=\mathrm{C}(\mathrm{Ph}) \mathrm{S}-\mathrm{R}]$ in free radical polymerization with reversible addition-fragmentation chain transfer (RAFT polymerization). Role of the free-radical leaving group (R), Macromolecules 2003, 36, 2256-2272.

[53] J. Chiefari, R. T. A. Mayadunne, C. L. Moad, G. Moad, E. Rizzardo, A. Postma, M. A. Skidmore, S. H. Thang, Thiocarbonylthio compounds $(S=C(Z) S-R)$ in free radical polymerization with reversible addition-fragmentation chain transfer (RAFT polymerization). Effect of the activating group Z, Macromolecules 2003, 36, 2273-2283.

[54] J. S. Wang, K. Matyjaszewski, Controlled Living Radical Polymerization - Atom-Transfer Radical Polymerization in the Presence of Transition-Metal Complexes, J Am Chem Soc 1995, 117, 5614-5615.

[55] C. Y. Lin, M. L. Coote, A. Gennaro, K. Matyjaszewski, Ab initio evaluation of the thermodynamic and electrochemical properties of alkyl halides and radicals and their mechanistic implications for atom transfer radical polymerization, J Am Chem Soc 2008, 130, $12762-12774$.

[56] K. Matyjaszewski, J. H. Xia, Atom transfer radical polymerization, Chem Rev 2001, 101, 29212990.

[57] K. Matyjaszewski, Atom Transfer Radical Polymerization (ATRP): Current Status and Future Perspectives, Macromolecules 2012, 45, 4015-4039.

[58] W. Tang, K. Matyjaszewski, Effects of initiator structure on activation rate constants in ATRP, Macromolecules 2007, 40, 1858-1863.

[59] W. Tang, K. Matyjaszewski, Effect of ligand structure on activation rate constants in ATRP, Macromolecules 2006, 39, 4953-4959.

[60] Y. Inoue, K. Matyjaszewski, New amine-based tripodal copper catalysts for atom transfer radical polymerization, Macromolecules 2004, 37, 4014-4021.

[61] W. A. Braunecker, N. V. Tsarevsky, A. Gennaro, K. Matyjaszewski, Thermodynamic Components of the Atom Transfer Radical Polymerization Equilibrium: Quantifying Solvent Effects, Macromolecules 2009, 42, 6348-6360.

[62] F. J. Xu, K. G. Neoh, E. T. Kang, Bioactive surfaces and biomaterials via atom transfer radical polymerization, Prog Polym Sci 2009, 34, 719-761.

[63] B. Zhao, W. J. Brittain, Polymer brushes: surface-immobilized macromolecules, Prog Polym Sci 2000, 25, 677-710.

[64] C. J. Fristrup, K. Jankova, S. Hvilsted, Surface-initiated atom transfer radical polymerization-a technique to develop biofunctional coatings, Soft Matter 2009, 5, 4623-4634. 
[65] A. Friebe, M. Ulbricht, Cylindrical Pores Responding to Two Different Stimuli via SurfaceInitiated Atom Transfer Radical Polymerization for Synthesis of Grafted Diblock Copolymers, Macromolecules 2009, 42, 1838-1848.

[66] C. A. Naini, S. Franzka, S. Frost, M. Ulbricht, N. Hartmann, Probing the Intrinsic Switching Kinetics of Ultrathin Thermoresponsive Polymer Brushes, Angew Chem Int Edit 2011, 50, 4513-4516.

[67] A. Kusumo, L. Bombalski, Q. Lin, K. Matyjaszewski, J. W. Schneider, R. D. Tilton, High capacity, charge-selective protein uptake by polyelectrolyte brushes, Langmuir 2007, 23, 4448-4454.

[68] B. V. Bhut, S. M. Husson, Dramatic performance improvement of weak anion-exchange membranes for chromatographic bioseparations, J Membrane Sci 2009, 337, 215-223.

[69] H. C. Dong, J. Y. Huang, R. R. Koepsel, P. L. Ye, A. J. Russell, K. Matyjaszewski, Recyclable Antibacterial Magnetic Nanoparticles Grafted with Quaternized Poly(2-(dimethylamino)ethyl methacrylate) Brushes, Biomacromolecules 2011, 12, 1305-1311.

[70] S. J. Yuan, F. J. Xu, S. O. Pehkonen, Y. P. Ting, K. G. Neoh, E. T. Kang, Grafting of Antibacterial Polymers on Stainless Steel Via Surface-Initiated Atom Transfer Radical Polymerization for Inhibiting Biocorrosion by Desulfovibrio desulfuricans, Biotechnol Bioeng 2009, 103, 268-281.

[71] J. H. Dai, Z. Y. Bao, L. Sun, S. U. Hong, G. L. Baker, M. L. Bruening, High-capacity binding of proteins by poly(acrylic acid) brushes and their derivatives, Langmuir 2006, 22, 4274-4281.

[72] N. Ayres, C. D. Cyrus, W. J. Brittain, Stimuli-responsive surfaces using polyampholyte polymer brushes prepared via atom transfer radical polymerization, Langmuir 2007, 23, 3744-3749.

[73] S. Tugulu, R. Barbey, M. Harms, M. Fricke, D. Volkmer, A. Rossi, H. A. Klok, Synthesis of poly(methacrylic acid) brushes via surface-initiated atom transfer radical polymerization of sodium methacrylate and their use as substrates for the mineralization of calcium carbonate, Macromolecules 2007, 40, 168-177.

[74] R. Dong, S. Krishnan, B. A. Baird, M. Lindau, C. K. Ober, Patterned biofunctional poly(acrylic acid) brushes on silicon surfaces, Biomacromolecules 2007, 8, 3082-3092.

[75] P. Jain, G. L. Baker, M. L. Bruening, Applications of Polymer Brushes in Protein Analysis and Purification, Annu Rev Anal Chem 2009, 2, 387-408.

[76] Z. Y. Zhang, J. C. Wang, Q. Tu, N. Nie, J. Sha, W. M. Liu, R. Liu, Y. R. Zhang, J. Y. Wang, Surface modification of PDMS by surface-initiated atom transfer radical polymerization of watersoluble dendronized PEG methacrylate, Colloid Surface B 2011, 88, 85-92.

[77] H. T. He, W. H. Jing, W. H. Xing, Y. Q. Fan, Improving protein resistance of alpha-Al2O3 membranes by modification with POEGMA brushes, Appl Surf Sci 2011, 258, 1038-1044.

[78] Y. Sui, Z. N. Wang, X. L. Gao, C. J. Gao, Antifouling PVDF ultrafiltration membranes incorporating PVDF-g-PHEMA additive via atom transfer radical graft polymerizations, J Membrane Sci 2012, 413, 38-47.

[79] W. Feng, J. L. Brash, S. P. Zhu, Non-biofouling materials prepared by atom transfer radical polymerization grafting of 2-methacryloloxyethyl phosphorylcholine: Separate effects of graft density and chain length on protein repulsion, Biomaterials 2006, 27, 847-855. 
[80] Z. Zhang, T. Chao, S. F. Chen, S. Y. Jiang, Superlow fouling sulfobetaine and carboxybetaine polymers on glass slides, Langmuir 2006, 22, 10072-10077.

[81] Y. Chang, W. J. Chang, Y. J. Shih, T. C. Wei, G. H. Hsiue, Zwitterionic Sulfobetaine-Grafted Poly(vinylidene fluoride) Membrane with Highly Effective Blood Compatibility via Atmospheric Plasma-Induced Surface Copolymerization, ACS Appl Mater Inter 2011, 3, 12281237.

[82] M. Ulbricht, Advanced functional polymer membranes, Polymer 2006, 47, 2217-2262.

[83] R. Ghosh, Protein separation using membrane chromatography: opportunities and challenges, J Chromatogr A 2002, 952, 13-27.

[84] D. K. Roper, E. N. Lightfoot, Separation of Biomolecules Using Adsorptive Membranes, J Chromatogr A 1995, 702, 3-26.

[85] A. Saxena, B. P. Tripathi, M. Kumar, V. K. Shahi, Membrane-based techniques for the separation and purification of proteins: An overview, Adv Colloid Interfac 2009, 145, 1-22.

[86] Q. Yang, N. Adrus, F. Tomicki, M. Ulbricht, Composites of functional polymeric hydrogels and porous membranes, J Mater Chem 2011, 21, 2783-2811.

[87] H. F. Zou, Q. Z. Luo, D. M. Zhou, Affinity membrane chromatography for the analysis and purification of proteins, J Biochem Bioph Meth 2001, 49, 199-240.

[88] X. F. Zeng, E. Ruckenstein, Membrane chromatography: Preparation and applications to protein separation, Biotechnol Progr 1999, 15, 1003-1019.

[89] C. Charcosset, Purification of proteins by membrane chromatography, J Chem Technol Biot 1998, 71, 95-110.

[90] E. Klein, Affinity membranes: a 10-year review, J Membrane Sci 2000, 179, 1-27.

[91] H. V. Adikane, G. J. Iyer, Chemical Modification of Ethyl Cellulose-Based Highly Porous Membrane for the Purification of Immunoglobulin G, Appl Biochem Biotech 2013, 169, 1026 1038.

[92] C. Boi, C. Algeri, G. C. Sarti, Preparation and Characterization of Polysulfone Affinity Membranes Bearing a Synthetic Peptide Ligand for the Separation of Murine Immunoglobulins, Biotechnol Progr 2008, 24, 1304-1313.

[93] L. Urbas, P. Brne, B. Gabor, M. Barut, M. Strlic, T. C. Petric, A. Strancar, Depletion of highabundance proteins from human plasma using a combination of an affinity and pseudoaffinity column, J Chromatogr A 2009, 1216, 2689-2694.

[94] M. Rucevic, J. G. Clifton, F. L. Huang, X. S. Li, H. Callanan, D. C. Hixson, D. Josic, Use of short monolithic columns for isolation of low abundance membrane proteins, J Chromatogr A 2006, 1123, 199-204.

[95] C. Boi, S. Dimartino, S. Hofer, J. Horak, S. Williams, G. C. Sarti, W. Lindner, Influence of different spacer arms on Mimetic Ligand (TM) A2P and B14 membranes for human IgG purification, J Chromatogr B 2011, 879, 1633-1640. 
[96] C. Boi, V. Busini, M. Salvalaglio, C. Cavallotti, G. C. Sarti, Understanding ligand-protein interactions in affinity membrane chromatography for antibody purification, $J$ Chromatogr $A$ 2009, 1216, 8687-8696.

[97] Y. Li, T. S. Chung, S. Y. Chan, High-affinity sulfonated materials with transition metal counterions for enhanced protein separation in dual-layer hollow fiber membrane chromatography, J Chromatogr A 2008, 1187, 285-288.

[98] B. Paull, C. O. Riordain, P. N. Nesterenko, Double gradient ion chromatography on a short carboxybetaine coated monolithic anion exchanger, Chem Commun 2005, 215-217.

[99] G. A. Tishchenko, M. Bleha, J. Skvor, T. Bostik, Effect of salt concentration gradient on separation of different types of specific immunoglobulins by ion-exchange chromatography on DEAE cellulose, J Chromatogr B 1998, 706, 157-166.

[100] J. W. Hu, C. H. Xie, R. J. Tian, Z. K. He, H. F. Zou, Separation of basic and acidic compounds by capillary electrochromatography using monolithic silica capillary columns with zwitterionic stationary phase, J Sep Sci 2007, 30, 891-899.

[101] C. V. Hoffmann, R. Pell, M. Lammerhofer, W. Lindner, Synergistic Effects on Enantioselectivity of Zwitterionic Chiral Stationary Phases for Separations of Chiral Acids, Bases, and Amino Acids by HPLC, Anal Chem 2008, 80, 8780-8789.

[102] Q. Yang, M. Ulbricht, Novel Membrane Adsorbers with Grafted Zwitterionic Polymers Synthesized by Surface-Initiated ATRP and Their Salt-Modulated Permeability and Protein Binding Properties, Chem Mater 2012, 24, 2943-2951.

[103] R. Ghosh, Separation of proteins using hydrophobic interaction membrane chromatography, J Chromatogr A 2001, 923, 59-64.

[104] Y. S. Chen, C. S. Chang, S. Y. Suen, Protein adsorption separation using glass fiber membranes modified with short-chain organosilicon derivatives, J Membrane Sci 2007, 305, 125-135.

[105] R. Ghosh, T. Wong, Effect of module design on the efficiency of membrane chromatographic separation processes, J Membrane Sci 2006, 281, 532-540.

[106] D. Q. Yu, X. J. Shang, R. Ghosh, Fractionation of different PEGylated forms of a protein by chromatography using environment-responsive membranes, J Chromatogr A 2010, 1217, 5595-5601.

[107] R. Ghosh, Fractionation of human plasma proteins by hydrophobic interaction membrane chromatography, J Membrane Sci 2005, 260, 112-118.

[108] R. Ghosh, L. Wang, Purification of humanized monoclonal antibody by hydrophobic interaction membrane chromatography, J Chromatogr A 2006, 1107, 104-109.

[109] R. Huang, K. Z. Mah, M. Malta, L. K. Kostanski, C. D. M. Filipe, R. Ghosh, Chromatographic separation of proteins using hydrophobic membrane shielded with an environmentresponsive hydrogel, J Membrane Sci 2009, 345, 177-182.

[110] M. Kuczewski, N. Fraud, R. Faber, G. Zarbis-Papastoitsis, Development of a Polishing Step Using a Hydrophobic Interaction Membrane Adsorber With a PER.C6 (R)-Derived Recombinant Antibody, Biotechnol Bioeng 2010, 105, 296-305. 
[111] N. Fraud, M. Kuczewski, G. Zarbis-Papastoitsis, M. Hirai, Hydrophobic Membrane Adsorbers for Large-Scale Downstream Processing, Biopharm Int 2009, 24-27.

[112] S. A. Piletsky, T. L. Panasyuk, E. V. Piletskaya, I. A. Nicholls, M. Ulbricht, Receptor and transport properties of imprinted polymer membranes - a review, J Membrane Sci 1999, 157, 263-278.

[113] X. J. Wang, Z. L. Xu, J. L. Feng, N. C. Bing, Z. G. Yang, Molecularly imprinted membranes for the recognition of lovastatin acid in aqueous medium by a template analogue imprinting strategy, J Membrane Sci 2008, 313, 97-105.

[114] J. MathewKrotz, K. J. Shea, Imprinted polymer membranes for the selective transport of targeted neutral molecules, J Am Chem Soc 1996, 118, 8154-8155.

[115] T. A. Sergeyeva, S. A. Piletsky, E. V. Piletska, O. O. Brovko, L. V. Karabanova, L. M. Sergeeva, A. V. El'skaya, A. P. F. Turner, In situ formation of porous molecularly imprinted polymer membranes, Macromolecules 2003, 36, 7352-7357.

[116] S. G. Wu, W. G. Tan, H. H. Xu, Protein molecularly imprinted polyacrylamide membrane: for hemoglobin sensing, Analyst 2010, 135, 2523-2527.

[117] Y. Lin, S. Q. Tang, X. Mao, L. Bao, Protein recognition via molecularly imprinted agarose gel membrane, J Biomed Mater Res A 2008, 85A, 573-581.

[118] H. Y. Wang, T. Kobayashi, N. Fujii, Molecular imprint membranes prepared by the phase inversion precipitation technique, Langmuir 1996, 12, 4850-4856.

[119] H. Y. Wang, T. Kobayashi, T. Fukaya, N. Fujii, Molecular imprint membranes prepared by the phase inversion precipitation technique .2. Influence of coagulation temperature in the phase inversion process on the encoding in polymeric membranes, Langmuir 1997, 13, 5396-5400.

[120] T. Kobayashi, H. Y. Wang, N. Fujii, Molecular imprint membranes of polyacrylonitrile copolymers with different acrylic acid segments, Anal Chim Acta 1998, 365, 81-88.

[121] M. Ramamoorthy, M. Ulbricht, Molecular imprinting of cellulose acetate-sulfonated polysulfone blend membranes for Rhodamine B by phase inversion technique, J Membrane Sci 2003, 217, 207-214.

[122] D. Silvestri, N. Barbani, M. L. Coluccio, C. Pegoraro, P. Giusti, C. Cristallini, G. Ciardelli, Poly(ethylene-co-vinyl alcohol) membranes with specific adsorption properties for potential clinical application, Separ Sci Technol 2007, 42, 2829-2847.

[123] H. Y. Wang, T. Kobayashi, N. Fujii, Surface molecular imprinting on photosensitive dithiocarbamoyl polyacrylonitrile membranes using photograft polymerization, J J Chem Technol Biot 1997, 70, 355-362.

[124] R. R. Chen, L. Qin, M. Jia, X. W. He, W. Y. Li, Novel surface-modified molecularly imprinted membrane prepared with iniferter for permselective separation of lysozyme, J Membrane Sci 2010, 363, 212-220.

[125] S. A. Piletsky, H. Matuschewski, U. Schedler, A. Wilpert, E. V. Piletska, T. A. Thiele, M. Ulbricht, Surface functionalization of porous polypropylene membranes with molecularly imprinted polymers by photograft copolymerization in water, Macromolecules 2000, 33, 3092-3098. 
[126] V. Kochkodan, W. Weigel, M. Ulbricht, Thin layer molecularly imprinted microfiltration membranes by photofunctionalization using a coated alpha-cleavage photoinitiator, Analyst 2001, 126, 803-809.

[127] K. Fukazawa, K. Ishihara, Fabrication of a cell-adhesive protein imprinting surface with an artificial cell membrane structure for cell capturing, Biosens Bioelectron 2009, 25, 609-614.

[128] D. Silvestri, C. Borrelli, P. Giusti, C. Cristallini, G. Ciardelli, Polymeric devices containing imprinted nanospheres: a novel approach to improve recognition in water for clinical uses, Anal Chim Acta 2005, 542, 3-13.

[129] D. Silvestri, N. Barbani, C. Cristallini, P. Giusti, G. Ciardelli, Molecularly imprinted membranes for an improved recognition of biomolecules in aqueous medium, J Membrane Sci 2006, 282, 284-295.

[130] S. Asliyuce, L. Uzun, A. Y. Rad, S. Unal, R. Say, A. Denizli, Molecular imprinting based composite cryogel membranes for purification of anti-hepatitis $B$ surface antibody by fast protein liquid chromatography, J Chromatogr B 2012, 889, 95-102.

[131] M, Ciampoli, N. Nardi, 5-Coordinated High-Spin Complexes of Bivalent Cobalt Nickel and Copper with Tris(2-Dimethylaminoethyl)Amine, Inorg Chem 1966, 5, 41-44.

[132] J. Queffelec, S. G. Gaynor, K. Matyjaszewski, Optimization of atom transfer radical polymerization using $\mathrm{Cu}(\mathrm{I}) / \mathrm{tris}$ (2-(dimethylamino)ethyl)amine as a catalyst, Macromolecules 2000,, 33, 8629-8639.

[133] Z. Y. Bao, M. L. Bruening, G. L. Baker, Control of the density of polymer brushes prepared by surface-initiated atom transfer radical polymerization, Macromolecules 2006,, 39, 5251-5258.

[134] K. Kato, E. Uchida, E. T. Kang, Y. Uyama, Y. Ikada, Polymer surface with graft chains, Prog Polym Sci 2003, 28, 209-259.

[135] U. Chatterjee, S. K. Jewrajka, B. M. Mandal, The beneficial effect of small amount of water in the ambient temperature atom transfer radical homo and block co-polymerization of methacrylates, Polymer 2005, 46, 1575-1582.

[136] A. K. Nanda, K. Matyjaszewski, Effect of $[\mathrm{bpy}] /[\mathrm{Cu}(\mathrm{I})]$ ratio, solvent, counterion, and alkyl bromides on the activation rate constants in atom transfer radical polymerization, Macromolecules 2003, 36, 599-604.

[137] H. G. Borner, K. Beers, K. Matyjaszewski, S. S. Sheiko, M. Moller, Synthesis of molecular brushes with block copolymer side chains using atom transfer radical polymerization, Macromolecules 2001, 34, 4375-4383.

[138] M. Retsch, A. Walther, K. Loos, A. H. E. Muller, Synthesis of dense poly(acrylic acid) brushes and their interaction with amine-functional silsesquioxane nanoparticles, Langmuir 2008, 24, 9421-9429.

[139] N. Bereli, M. Andac, G. Baydemir, R. Say, I. Y. Galaev, A. Denizli, Protein recognition via ioncoordinated molecularly imprinted supermacroporous cryogels, J Chromatogr A 2008, 1190, 18-26.

[140] http://www.rcsb.org/pdb/explore/jmol.do?structureld=2LYZ\&opt=3\&bionumber=1. 
[141] H. H. P. Yiu, C. H. Botting, N. P. Botting, P. A. Wright, Size selective protein adsorption on thiolfunctionalised SBA-15 mesoporous molecular sieve, Phys Chem Chem Phys 2001, 3, 29832985.

[142] S. Mendez, J. G. Curro, J. D. McCoy, G. P. Lopez, Computational modeling of the temperatureinduced structural changes of tethered poly( $\mathrm{N}$-isopropylacrylamide) with self-consistent field theory, Macromolecules 2005, 38, 174-181.

[143] H. Yim, M. S. Kent, S. Mendez, G. P. Lopez, S. Satija, Y. Seo, Effects of grafting density and molecular weight on the temperature-dependent conformational change of poly $(\mathrm{N}$ isopropylacrylamide) grafted chains in water, Macromolecules 2006, 39, 3420-3426.

[144] W. J. Brittain, S. Minko, A structural definition of polymer brushes, J Polym Sci Pol Chem 2007, 45, 3505-3512.

[145] T. Kawai, K. Sugita, K. Saito, T. Sugo, Extension and shrinkage of polymer brush grafted onto porous membrane induced by protein binding, Macromolecules 2000, 33, 1306-1309.

[146] H. Okuno, T. Kitano, H. Yakabe, M. Kishimoto, B. A. Deore, H. Siigi, T. Nagaoka, Characterization of overoxidized polypyrrole colloids imprinted with L-lactate and their application to enantioseparation of amino acids, Anal Chem 2002, 74, 4184-4190.

[147] A. Nematollahzadeh, W. Sun, C. S. A. Aureliano, D. Lutkemeyer, J. Stute, M. J. Abdekhodaie, A. Shojaei, B. Sellergren, High-Capacity Hierarchically Imprinted Polymer Beads for Protein Recognition and Capture, Angew Chem Int Edit 2011, 50, 495-498.

[148] M. E. Byrne, K. Park, N. A. Peppas, Molecular imprinting within hydrogels, Adv Drug Deliver Rev 2002, 54, 149-161.

[149] Z. A. Lin, F. Yang, X. W. He, X. M. Zhao, Y. K. Zhang, Preparation and evaluation of a macroporous molecularly imprinted hybrid silica monolithic column for recognition of proteins by high performance liquid chromatography, J Chromatogr A 2009, 1216, 8612-8622.

[150] M. Zayats, M. Kanwar, M. Ostermeier, P. C. Searson, Molecular Imprinting of Maltose Binding Protein: Tuning Protein Recognition at the Molecular Level, Macromolecules 2011, 44, 3966 3972.

[151] M. Harper, F. Lema, G. Boulot, R. J. Poljak, Antigen-Specificity and Cross-Reactivity of Monoclonal Antilysozyme Antibodies, Mol Immunol 1987, 24, 97-108.

[152] Y. Hoshino, T. Kodama, Y. Okahata, K. J. Shea, Peptide Imprinted Polymer Nanoparticles: A Plastic Antibody, J Am Chem Soc 2008, 130, 15242-15243.

[153] S. H. Lee, Y. Hoshino, A. Randall, Z. Y. Zeng, P. Baldi, R. A. Doong, K. J. Shea, Engineered Synthetic Polymer Nanoparticles as IgG Affinity Ligands, J Am Chem Soc 2012, 134, 1576515772.

[154] Q. Y. Li, Z. F. Cui, D. S. Pepper, Fractionation of HSA and IgG by gas sparged ultrafiltration, J Membrane Sci 1997, 136, 181-190.

[155] D. Song, D. Forciniti, Effects of cosolvents and pH on protein adsorption on polystyrene latex: A dynamic light scattering study, J Colloid Interf Sci 2000, 221, 25-37. 
[156] A. K. Hunter, G. Carta, Protein adsorption on novel acrylamido-based polymeric ionexchangers. IV. Effects of protein size on adsorption capacity and rate, J Chromatogr A 2002, 971, 105-116.

[157] N. Singh, J. Wang, M. Ulbricht, S. R. Wickramasinghe, S. M. Husson, Surface-initiated atom transfer radical polymerization: A new method for preparation of polymeric membrane adsorbers, J Membrane Sci 2008, 309, 64-72.

[158] N. Singh, Z. Chen, N. Tomer, S. R. Wickramasinghe, N. Soice, S. M. Husson, Modification of regenerated cellulose ultrafiltration membranes by surface-initiated atom transfer radical polymerization, J Membrane Sci 2008, 311, 225-234.

[159] D. Matulis, I. Rouzina, V. A. Bloomfield, Thermodynamics of cationic lipid binding to DNA and DNA condensation: Roles of electrostatics and hydrophobicity, J Am Chem Soc 2002, 124, 7331-7342.

[160] J. Kobayashi, A. Kikuchi, K. Sakai, T. Okano, Aqueous chromatography utilizing hydrophobicity-modified anionic temperature-responsive hydrogel for stationary phases, $J$ Chromatogr A 2002, 958, 109-119.

[161] D. E. Owens, Y. C. Jian, J. E. Fang, B. V. Slaughter, Y. H. Chen, N. A. Peppas, Thermally responsive swelling properties of polyacrylamide/poly(acrylic acid) interpenetrating polymer network nanoparticles, Macromolecules 2007, 40, 7306-7310.

[162] H. J. Dai, Q. Chen, H. L. Qin, Y. Guan, D. Y. Shen, Y. Q. Hua, Y. L. Tang, J. Xu, A temperatureresponsive copolymer hydrogel in controlled drug delivery, Macromolecules 2006, 39, 65846589.

[163] Z. D. Hua, Z. Y. Chen, Y. Z. Li, M. P. Zhao, Thermosensitive and salt-sensitive molecularly imprinted hydrogel for bovine serum albumin, Langmuir 2008, 24, 5773-5780.

[164] T. A. Sergeyeva, H. Matuschewski, S. A. Piletsky, J. Bendig, U. Schedler, M. Ulbricht, Molecularly imprinted polymer membranes for substance-selective solid-phase extraction from water by surface photo-grafting polymerization, J Chromatogr A 2001, 907, 89-99.

[165] J. Pontius, J. Richelle, S. J. Wodak, Deviations from standard atomic volumes as a quality measure for protein crystal structures, J Mol Biol 1996, 264, 121-136.

[166] J. Wang, F. Dismer, J. Hubbuch, M. Ulbricht, Detailed analysis of membrane adsorber pore structure and protein binding by advanced microscopy, J Membrane Sci 2008, 320, 456-467.

[167] M. Bompart, K. Haupt, Molecularly Imprinted Polymers and Controlled/Living Radical Polymerization, Aust J Chem 2009, 62, 751-761.

[168] L. Qin, X. W. He, X. Yuan, W. Y. Li, Y. K. Zhang, Molecularly imprinted beads with double thermosensitive gates for selective recognition of proteins, Anal Bioanal Chem 2011, 399, 3375-3385.

[169] B. Ruckert, A. J. Hall, B. Sellergren, Molecularly imprinted composite materials via inifertermodified supports, J Mater Chem 2002, 12, 2275-2280.

[170] S. Lakshmi, A. Jayakrishnan, Photocross-linking of dithiocarbamate-substituted PVC reduces plasticizer migration, Polymer 1998, 39, 151-157. 
[171] D. R. Kryscio, N. A. Peppas, Surface imprinted thin polymer film systems with selective recognition for bovine serum albumin, Anal Chim Acta 2012, 718, 109-115.

[172] G. Alpdogan, S. Sungur, AAS and spectrophotometric methods for the determination metoprolol tartrate in tablets, Spectrochim Acta A 1999, 55, 2705-2709.

[173] B. G. Jeliazkova, M. A. Doicheva, Charge-transfer photochemistry of copper(II) dithiocarbamate mixed-ligand complexes, Polyhedron 1996, 15, 1277-1282. 


\section{List of abbreviations}

AA

AAm

ACBN

Al

AMPS

APBA

APMA. $\mathrm{HCl}$

ARPCs

ATRP

BET

$\mathrm{BHb}$

BIB

BSA

CBMA

DEPN

DMF

DMPAA

DTPA

EDC

EDGMA

EDTA

HEMA

$\mathrm{HGH}$

$\mathrm{HMb}$

HSA

IgG

ISET

MAA

MBAA two dimensional

three dimensional

acrylic acid

acrylamide

1,1'-azobis-(cyclohexanecarbonitrile)

ATRP initiator

2-Acrylamido-2-methylpropansulfonic acid

aminophenylboronic acid

$\mathrm{N}$-(3-aminopropyl)methacrylamide hydrochloride

assistant recognition polymer chains

atom transfer radical polymerization

Brunauer, Emmett and Teller

bovine hemoglobin

$\alpha$-bromoisobutyryl bromide

bovine serum albumin

carboxybetaine methyacrylate

$N$-(1-diethylphosphono-2,2-dimethylpropyl)- $N$-oxyl

dimethylformamide

$\mathrm{N}, \mathrm{N}$-dimethylaminopropyl acrylamide

diethylene triamine pentaacetic acid

$N$-(3-dimethylaminopropyl)- $N$ '-ethylcarbodiimide hydrochloride ethylene glycol dimethacrylate

ethylene diaminetetraacetic acid

2-hydroxlyethyl methacrylate

hunamn growth hormone

horse myoglobin

albumin from human serum

immunglobulin G

inner-sphere electron transfer

methacrylic acid

$\mathrm{N}, \mathrm{N}^{\prime}$-methylenebisacrylamide 


\begin{tabular}{|c|c|}
\hline $\mathrm{Me}_{6}$ TREN & Tris[2-(dimethylamino)ethyl]amine \\
\hline $\mathrm{MeCN}$ & acetonitrile \\
\hline MF & microfiltration \\
\hline MIP & molecularly imprinted polymer \\
\hline MPC & methacryloyloxyethyl phosphorylcholine \\
\hline NF & nanofiltration \\
\hline NIP & non imprinted polyemer \\
\hline NIPAAm & $\mathrm{N}$-isopropylacrylamide \\
\hline NHS & $N$-hydroxysuccinimide \\
\hline NMP & nitroxide-mediated polymerization \\
\hline OSET & outer-sphere electron transfer \\
\hline PET & polyethylene terephthalate \\
\hline PDMS & poly(dimethylsiloxane) \\
\hline $\mathrm{pl}$ & isoelectric point \\
\hline PMAA & poly(methacrylic acid) \\
\hline PMDETA & $N, N, N^{\prime}, N^{\prime \prime}, N^{\prime \prime}$-pentamethyldiethylenetriamine \\
\hline PMMA & poly(methyl methacrylate) \\
\hline POEGMA & poly(oligo(ethelyene glycol) methaycrylate) \\
\hline PPEGMA & poly(poly(ethelyene glycol) methaycrylate) \\
\hline PVDF & poly(vinylidene fluoride) \\
\hline PVBC & poly(4-vinylbenzyl chrloride) \\
\hline RAFT & reversible addition fragmentation chain transfer polymerization \\
\hline RFGD & radio frequency glow-discharge \\
\hline RO & reverse osmosis \\
\hline SBMA & sulfobetaine methacrylate \\
\hline SDS & sodium dodecyl sulfate \\
\hline SI-ATRP & surface initiated ATRP \\
\hline TABA & tert.-butyl ammonium bromide \\
\hline$t \mathrm{BMA}$ & tert.-butyl methacrylate \\
\hline TEA & triethylamine \\
\hline TE PET & tracked etched PET \\
\hline TEMPO & 2,2,6,6-tetramethyl-1-piperidynyl- $\mathrm{N}$-oxy \\
\hline
\end{tabular}


TFA

THF

TIPNO

TMS

UF

XPS trifluoroacetic acid

tetrahydrofuran

2,2,5-trimethyl-4-phenyl-3-azahexane-3-nitroxide trimethylsilanoxyethyl

ultrafiltration

X-ray photoelectron spectroscopy 


\section{List of tables}

Table 3.1 ATRP conditions engaged in this work.

Table 4.1 Comparison of the data from manufacturer and measurement in laboratory.

Table 4.2 Content of elements from elemental analysis of copper(I) chloride.

Table 4.3 Result of ATRP in $1.5 \mathrm{~mol} / \mathrm{L} t \mathrm{BMA}$ with a catalyst system of [tBMA]:[CuCl]:

$\left[\mathrm{CuCl}_{2}\right]:[\mathrm{Bpy}]=75: 1: 0.1: 2.75$ at ambient temperature for $4 \mathrm{~h}$.

Table 4.4 Information on Lyz and CyC.

Table 4.5 Information on IgG and HSA.

Table 4.6 Comparison of cellulose membrane and TE PET 400 membrane. 


\section{List of schemes}

Scheme 1.1 Immobilization of ATRP initiator on TE PET surface.

Scheme 1.2 SI-ATRP of PtBMA and subsequent hydrolysis to PMAA on TE PET surface.

Scheme 2.1 Mechanism of radical polymerization.

Scheme 2.2 Mechanism of radical activation and deactivation process by NMP and ATRP.

Scheme 2.3 Mechanism of radical transfer by RAFT.

Scheme 2.4 Mechanism of NMP by TEMPO initiation.

Scheme 2.5 Four examples of nitroxide employed by NMP.

Scheme 2.6 Mechanism of RAFT with a thiocarbonylthio transfer agent.

Scheme 2.7 Structural features of thiocarbonylthio RAFT agent and the intermediate formed on radical addition.

Scheme 2.8 For $\mathrm{R}$, rates of fragmentation decrease from left to right. For $\mathrm{Z}$, addition rates decrease and fragmentation rate increase from left to right.

Scheme 4.1 Complex state of Bpy with $\mathrm{CuBr}$ in the solvents with different polarities.

Scheme 4.2 Oxidation of copper (I) chloride in air.

Scheme 4.3 Surface with two initiators: benzyl chloride for ATRP and dithiocarbamate for UV-initiation (RAFT).

Scheme 4.4 Hydrolysis of dithiocarbamate group in aqueous solution. 


\section{List of figures}

Figure 1.1 Protein imprinting via two step grafting using SI-ATRP and UV-initiated grafting/crosslinking copolymerization on track-etched PET membrane surface.

Figure 2.1 Schematic representation of molecular imprinting process

Figure $2.2 \quad 20 \mathrm{~L}$ - $\alpha$-amino acids.

Figure 2.3 Four level structure of proteins.

Figure 2.4 Surface imprinting of protein by Shi via RFGD plasma deposition.

Figure 2.5 Protocol for epitope imprinting for protein $\mathrm{CyC}$ recognition by Shea.

Figure 2.6 Protein scaffold imprinting with assistant recognition polymer chains from Mi and coworkers.

Figure 2.7 Mechanism of ATRP and the possibility of the substrates.

Figure 2.8 Comparison of the free energies during an ISET and OSET process for the reaction of bromoacetonitrile with $\mathrm{Cu}$ I/TPMA catalyst in MeCN at $25^{\circ} \mathrm{C}$

Figure 2.9 ATRP activation rate constants for various initiators with $\mathrm{Cu}(\mathrm{I}) \mathrm{X} / \mathrm{PMDETA}(\mathrm{X}=$ $\mathrm{Br}$ or $\mathrm{Cl}$ ) in $\mathrm{MeCN}$ at $35{ }^{\circ} \mathrm{C}$. $3^{\circ}$ : red; $2^{\circ}$ : blue; $1^{\circ}$ : black; isothiocyanate/thiocyanate: left half-filled; chloride: open; bromide: filled; iodide: bottom half-filled; amide: $\mathbf{\nabla}$; benzyl: $\boldsymbol{\Delta}$; ester: $\square$; nitrile: o; phenyl ester: $\diamond$.

Figure 2.10 ATRP activation rate constants for various ligands with EtBriB in the presence of CulBr in $\mathrm{MeCN}$ at $35{ }^{\circ} \mathrm{C}$. N2: red; N3: black; N4: blue; amine/imine: solid; pyridine: open; mixed: left-half solid; linear: $\square$; branched: $\boldsymbol{\Delta}$; cyclic: 0.

Figure 2.11 Examples of controlled macromolecular architecture in polymers prepared by ATRP: a, controlled composition/microstructure; b, controlled topology; $c$, controlled functionality.

Figure 2.12 Immobilization of ATRP initiators on organic/polymeric substrates.

Figure 2.13 Immobilization of ATRP initiators on inorganic and metallic substrates.

Figure 2.14 Schematic illustration of different pressure-drive membrane processed according to size of pores in the selective barrier and size of exemplaric targets for separation.

Figure 2.15 Schematic presentation of different membrane adsorber geometries.

Figure 3.1 Schematic illustration of protein imprinting process. 
Figure 4.1 Pore size distribution measured by liquid dewetting permprometry for TET PET400 basis membrane

Figure 4.2 SEM image of PET400 basis membrane: top view (left) and cross-section (right).

Figure 4.3 Immobilization of ATRP initiator on TE PET membrane surface.

Figure 4.4 Carboxyl group densities on the PET membrane surface after reaction with ethanolamine ("amination") with different activation times.

Figure 4.5 XPS spectra of the nitrogen region of different membranes: black ... base TE PET membrane; green ... TE PET after amination with $0.5 \mathrm{~h}$ activation time; red ... TE PET after amination with $6 \mathrm{~h}$ activation time.

Figure 4.6 XPS spectra of the bromine region of different membranes: blue ... base TE PET membrane; green ... TE PET after $\alpha$-bromoisobutyryl group immobilization with previously $0.5 \mathrm{~h}$ activiation time; red ... TE PET after $\alpha$-bromoisobutyryl group immobilization with previously $6 \mathrm{~h}$ activation time.

Figure 4.7 Degrees of grafting for PtBMA on PET membrane surface versus the reaction time of ATRP in $2 \mathrm{~mol} / \mathrm{L} t \mathrm{BMA}$ solution in DMF with a catalyst system of $[t B M A]:[\mathrm{CuCl}]:\left[\mathrm{Me}_{6}\right.$ TREN $]=100: 1: 1$.

Figure 4.8 Degrees of grafting for PtBMA on PET membrane surface versus the different ligand of ATRP in $2 \mathrm{~mol} / \mathrm{L} t \mathrm{BMA}$ solution in acetone / water solution mixture $(\mathrm{V}: \mathrm{V}=9: 1)$ with a catalyst system of $[t \mathrm{BMA}]:[\mathrm{CuCl}]:[\mathrm{Bpy}]=100: 1: 2$ and [tBMA]:[CuCl]:[Me 6 TREN/PMDETA] $=100: 1: 1$ at ambient temperature for $6 \mathrm{~h}$.

Figure 4.9 Degree of grafting for PtBMA on PET membrane surface versus the different $t B M A$ concentrations in acetone / water solution mixture $(V: V=9: 1)$ with a catalyst system of $[$ tBMA]:[CuCl]:[Bpy] $=100 / 75 / 50: 1: 2$ at ambient temperature for $6 \mathrm{~h}$.

Figure 4.10 Degree of grafting for PtBMA on PET membrane surface versus the Different ratios of $\mathrm{Bpy}$ to $\mathrm{Cu}(\mathrm{l})$ in $1.5 \mathrm{~mol} / \mathrm{L}$ tBMA acetone / water solution mixture $(\mathrm{V}: \mathrm{V}=9: 1)$ with a catalyst system of $[$ tBMA] $:[\mathrm{CuCl}]:[\mathrm{Bpy}]=75: 1: 2 / 2.5 / 3$ at ambient temperature for $6 \mathrm{~h}$.

Figure 4.11 Degree of grafting for PtBMA on PET membrane surface versus the different solvents acetone / water and acetonitirole / water solution mixture ( $\mathrm{V}: \mathrm{V}=9: 1)$ 
in $1.5 \mathrm{~mol} / \mathrm{L} t \mathrm{BMA}$ with a catalyst system of $[\mathrm{tBMA}]:[\mathrm{CuCl}]:[\mathrm{Bpy}]=75: 1: 2$ at ambient temperature for $6 \mathrm{~h}$.

Figure 4.12 Degree of grafting for PtBMA on PET membrane surface versus ATRP reaction time in acetonitirole / water solution mixture $(\mathrm{V}: \mathrm{V}=9: 1)$ in $1.5 \mathrm{~mol} / \mathrm{L} t \mathrm{BMA}$ with a catalyst system of $[t \mathrm{BMA}]:[\mathrm{CuCl}]:[\mathrm{Bpy}]=75: 1: 2.5$ (square) and $[$ tBMA]:[CuCl]:[CuCl 2$]:[\mathrm{Bpy}]=$ 75:1:0.1:2.75 (point) at ambient temperature.

Figure 4.13 Mass lose by hydrolysis of PtBMA to PMAA on PET membrane versus DG of PtBMA. The hydrolysis was taken placed in dichloromethane containing $1 \%$ methansulfonic acid at room temperature for $15 \mathrm{~min}$. Open columns represent experimental results and open ones represent the theoretical values.

Figure 4.14 Lysozyme binding capacity versus the DG of PMAA, the DG $=0$ is the PtBMA-gmembrane without hydrolysis. Protein adsorption condition: $2.0 \mathrm{~g} / \mathrm{L} \mathrm{Lyz} \mathrm{in} \mathrm{PBS}$ $(\mathrm{pH}=7, \mathrm{C}=0.066 \mathrm{~mol} / \mathrm{L})$, room temperature, $24 \mathrm{~h}$.

Figure 4.15 Mass of PET base membranes versus diferent solvents, open columns are the original membrane mass and solid columns are the mass after $24 \mathrm{~h}$ soaking with shaking $120 \mathrm{rmp}$ at room temperature.

Figure 4.16 Mass lose of PET basis membranes versus the volume ratio of TFA in MeCN, open columns are the original membrane mass and solid columns are the mass after $24 \mathrm{~h}$ soak with shaking $120 \mathrm{rpm}$ at $50^{\circ} \mathrm{C}$.

Figure 4.17 Mass lost percent of grafted polymer by hydrolysis from PtBMA to PMAA on PET membranes in $50 \%$ TFA solution versus the conditions in form "solvent, time".

Figure 4.18 Degree of grafting for PtBMA on PET membrane surface (squares) versus the reaction time of ATRP in $1.5 \mathrm{~mol} / \mathrm{L}$ tBMA solution in $\mathrm{MeCN} /$ water solution mixture $(\mathrm{V}: \mathrm{V}=9: 1)$ with a catalyst system of $[t \mathrm{BMA}]:[\mathrm{CuCl}]:\left[\mathrm{CuCl}_{2}\right]:[\mathrm{Bpy}]=$ 75:1:0.1:2.75 at ambient temperature; PET-g-PMAA (rhombuses) was obtained via hydrolysis of the PET-g-PtBMA membranes in 50\% TFA solution in MeCN / water mixture $(\mathrm{V}: \mathrm{V}=1: 1)$ at $50^{\circ} \mathrm{C}$ for $18 \mathrm{~h}$.

Figure 4.19 Contact angle results for membranes after different stages of functionalization. PET-Br corresponds to a membrane after ATRP initiator 
immobilization; PtBMA and PMAA correspond to the membranes grafted with PtBMA (4 h ATRP; $2.26 \mu \mathrm{g} / \mathrm{cm}^{2}$ ) and PMAA $\left(1.17 \mu \mathrm{g} / \mathrm{cm}^{2}\right)$, respectively.

Figure 4.20 Pore distribution versus the pore diameter of PET membranes: $\mathrm{Al}$ is the membrane after ATRP initiator immobilization PtBMA and PMAA correspond to the membranes grafted with PtBMA (2 h ATRP; $0.57 \mu \mathrm{g} / \mathrm{cm}^{2}$ ) and PMAA $\left(0.36 \mu \mathrm{g} / \mathrm{cm}^{2}\right)$, respectively.

Figure 4.21 Hydrodynamic layer thickness of grafted PMAA on PET pore wall with DG 0.74 $\mu \mathrm{g} / \mathrm{cm}^{2}$ in different solutions. "Collapse" result represents the dry layer thickness measure by PMI, pH varied with the values 3, 7, and 10.2. Addition of $\mathrm{NaCl}$ in PBS $(0.1 \mathrm{~mol} / \mathrm{L})$ and mixture of water and ethanol were also engaged to investigated the polyelectolyte's conformation.

Figure 4.22 Schematic representation of the PMAA chain's conformation on PET surface in PBS as a function of $\mathrm{pH}$ and salt concentration; effect on the deprotonation of carboxyl group by mixing of organic ethanol into pure water.

Figure 4.23 Influence of UV-irradiation time on the DG of grafted crosslinked PAAm PAAm on PET-g-PMAA membrane surface $\left(D_{\mathrm{PMAA}}=1.31 \mu \mathrm{g} / \mathrm{cm}^{2}\right): 0.7 \mathrm{~mol} / \mathrm{L}$ UVfunctional monomer $A A m$ and MBAA with molar ratio $n_{A A m}: n_{M B A A}=9=1$, i. e. degree of crosslinking 10\%, UV-intensity about $72 \mathrm{~mW} / \mathrm{cm}^{2}$.

Figure 4.24 Influence of UV-irradiation time on the DG of grafted crosslinked PAAm on PET-g-PMAA membrane surface $\left(D_{\text {PMAA }}=1.31 \mu \mathrm{g} / \mathrm{cm}^{2}\right): 0.7 \mathrm{~mol} / \mathrm{L}$ UVfuctional monomer $A A m$ and $M B A A$ with mol ratio $n_{A A m}: n_{M B A A}=9: 1$, i.e. degree of crosslinking 10\%, UV-intensity about $24 \mathrm{~mW} / \mathrm{cm}^{2}$.

Figure 4.25 Degree of grafting for PAAm on PET-g-PMAA membrane surface $\left(D G_{P M A A}=1.43 \mu \mathrm{g} / \mathrm{cm}^{2}\right)$ versus the concentration of the functional monomer AAm and MBAA with degree of crosslinking 10\%, irradiation under the UVintensity $24 \mathrm{~mW} / \mathrm{cm}^{2}$ for $10 \mathrm{~min}$.

Figure 4.26 Degree of grafting for PAAm PET-g-PMAA membrane surface $\left(D G_{P M A A}=1.43 \mu \mathrm{g} / \mathrm{cm}^{2}\right)$ versus the irradiation time: $0.3 \mathrm{~mol} / \mathrm{L}$ AAm and MBAA with degree of crosslinking 10\%, irradiation under the UV-intensity 24 $\mathrm{mW} / \mathrm{cm}^{2}$. 
Figure 4.27 Degree of grafting for PAAm on Al immobilized membrane surface versus the irradiation time: $0.7 \mathrm{~mol} / \mathrm{L}$ AAm, irradiation under the UV-intensity 24 $\mathrm{mW} / \mathrm{cm}^{2}$.

Figure 4.28 Structure of Lyz colored by amino acid (PDB ID: 2LYZ

Figure 4.29 Hydrodynamic layer thickness of grafted polymer on the PET pore walls of different membranes in water / ethanol $(1: 1, \mathrm{pH}=7.2)$ : Al corresponds to a membrane after ATRP initiator immobilization; TE PET with grafted PtBMA (after $3 \mathrm{~h}$ ATRP) and the subsequently obtained grafted PMAA had DG values of $1.62 \mu \mathrm{g} / \mathrm{cm}^{2}$ and $0.94 \mu \mathrm{g} / \mathrm{cm}^{2}$, respectively; the same UV-initiated grafting/crosslinking conditions, $10 \%$ crosslinking degree (i.e., MBAA fraction in the monomer mixture) and 20 min UV time, had been used for MIP and NIP synthesis using PET-g-PMAA with DG $=0.94 \mu \mathrm{g} / \mathrm{cm}^{2}$.

Figure 4.30 Influence of UV-grafting/crosslinking time on the properties of Lys-imprinted TE PET membranes: the Lyz and CYC binding capacities (open and solid columns) and resulting Lyz vs. CyC selectivity (squares) of the different MIP membranes with the same length of scaffold chains (ATRP: $D_{\text {PMAA }}=1.29$ $\mu \mathrm{g} / \mathrm{cm}^{2}$; UV: $0.3 \mathrm{~mol} / \mathrm{L}$ functional monomer; $A A m: M B A A=9: 1$, i.e. degree of crosslinking 10\%; UV-intensity about $24 \mathrm{~mW} / \mathrm{cm}^{2}$ ).

Figure 4.31 Effect of scaffold chain length (DG) on the Lyz and CyC binding capacities (open and solid columns) and resulting Lyz vs. CyC selectivity (squares) of the different MIP membranes. The UV-initiated grafting/crosslinking was carried out in $0.3 \mathrm{~mol} / \mathrm{L}$ functional monomers (AAM:MBAA = 9:1, i.e. degree of cross linking $=10 \%$ ) for 20 min under the UV-intensity about $20 \mathrm{~mW} / \mathrm{cm}^{2}$.

Figure 4.32 Effect of crosslinking degree on protein binding capacity and Lyz to CyC selectivity of MIP membranes with different scaffold DG. Open and solid columns represent the binding capacities of Lyz and CyC, respectively, squares the Lyz to CyC selectivity. Black represents the series of MIP membranes with scaffold DG $=0.75 \mu \mathrm{g} / \mathrm{cm}^{2}$, blue represents the series of MIP membranes with scaffold DG $=1.06 \mathrm{~g} / \mathrm{cm}^{2}$.

Figure 4.33 Lyz (open columns) and CyC (solid columns) binding capacities and Lyz to CyC selectivities (squares) for MIP, NIP and scaffold membranes (scaffold DG = $0.75 \mu \mathrm{g} / \mathrm{cm}^{2} ; 12 \%$ crosslinking degree). 
Figure 4.34 Lyz (open columns) and CyC (solid columns) binding capacities and Lyz to CyC selectivity (squares) of MIP membranes (scaffold DG $=0.75 \mu \mathrm{g} / \mathrm{cm}^{2} ; 12 \%$ crosslinking degree) obtained in single protein experiments $(0.2 \mathrm{~g} / \mathrm{L})$ and from in situ separation of a $1: 1$ protein mixture $(0.1+0.1 \mathrm{~g} / \mathrm{L})$ at $\mathrm{pH}=7.0$.

Figure 4.35 Lyz binding isotherms of MIP (solid squares) and NIP (solid circles) membranes as well as curves obtained by fitting the data to the Langmuir model.

Figure 4.36 Effect of ionic strength on Lyz binding to MIP (squares) and NIP (circles) membranes (protein concentration $0.2 \mathrm{~g} / \mathrm{L}$, phosphate buffer $\mathrm{pH}=7$ ).

Figure 4.37 Chemical structures of additive functional monomers during the UVpolymerization.

Figure 4.38 Lyz (open columns), CyC (solid columns) binding capacities and selectivity of Lyz to CyC (squares) of MIP membranes (scaffold DG PMAA $_{20} \approx 0.80 \mu \mathrm{g} / \mathrm{cm}^{2}$ ), UVgrafting/crosslinking: $\quad 0.3 \mathrm{~mol} / \mathrm{L}$ monomer concentration with AAm:MBAA:additive $=7.8: 1.2: 1$, irradiation under $20 \mathrm{~mW} / \mathrm{cm}^{2}$ for $20 \mathrm{~min}$.

Figure 4.39 Structure of IgG with heavy chains in blue and green and light chains in orange and red.

Figure 4.40 Eluted IgG after crosslinking (dash open columns), rebinding IgG (open columns), and HSA (solid columns) binding capacities and IgG to HSA selectivity (squares) of MIP membranes (scaffold DG PMAA $_{2} \approx 0.58 \mu \mathrm{g} / \mathrm{cm}^{2}$ ), UVgrafting/crosslinking: $0.3 \mathrm{~mol} / \mathrm{L}$ monomer concentration with $\mathrm{AAm}: \mathrm{MBAA}=$ $9: 1$, i.e. crosslinking degree $=10 \%$, irradiation under $20 \mathrm{~mW} / \mathrm{cm}^{2}$ for 10 to 15 $\min$.

Figure 4.41 IgG (open columns), and HSA (solid columns) binding capacities and IgG to HSA selectivity (squares) of MIP membranes (scaffold DG PMAA $_{20} 0.55 \mu \mathrm{g} / \mathrm{cm}^{2}$ ), UV-grafting/crosslinking: $0.3 \mathrm{~mol} / \mathrm{L}$ monomer concentration of AAm and MBAA with crosslinking degree $=10 \%$ and $12 \%$, irradiation under $20 \mathrm{~mW} / \mathrm{cm}^{2}$ for $12.5 \mathrm{~min}$.

Figure 4.42 IgG (open columns), and HSA (solid columns) binding capacities and IgG to HSA selectivity (squares) of MIP membranes (scaffold DG PMAA $_{2} \approx 0.55$ and 0.97 $\mu \mathrm{g} / \mathrm{cm}^{2}$ ), UV-grafting/crosslinking: $0.3 \mathrm{~mol} / \mathrm{L}$ monomer concentration of AAm and MBAA with crosslinking degree $=12 \%$, irradiation under $20 \mathrm{~mW} / \mathrm{cm}^{2}$ for $12.5 \mathrm{~min}$. 
Figure 4.43 Pore size distribution measured by liquid dewetting permprometry for cellulose base membrane.

Figure 4.44 SEM image of base membrane

Figure 4.45 DG for PtBMA on cellulose membrane versus the Al immobilization time in THF, ATRP: $1.5 \mathrm{~mol} / \mathrm{L}$ tBMA solution in $\mathrm{MeCN} /$ water solution mixture $(\mathrm{V}: \mathrm{V}=$ 9:1) with a catalyst system of $[t \mathrm{BMA}]:[\mathrm{CuCl}]:\left[\mathrm{CuCl}_{2}\right]:[\mathrm{Bpy}]=75: 1: 0.1: 2.75$ at ambient temperature for $5 \mathrm{~h}$.

Figure 4.46 DG for PtBMA on cellulose membrane versus the ratio of $\alpha$-bromoisobutyryl bromide (BIB) in mixture of BIP and propionyl bromide in MeCN (squares) and THF (points), ATRP: $1.5 \mathrm{~mol} / \mathrm{L}$ tBMA solution in MeCN / water solution mixture $(\mathrm{V}: \mathrm{V}=9: 1)$ with a catalyst system of $[t \mathrm{BMA}]:[\mathrm{CuCl}]:\left[\mathrm{CuCl}_{2}\right]:[\mathrm{Bpy}]=$ 75:1:0.1:2.75 at ambient temperature for $5 \mathrm{~h}$.

Figure 4.47 DG for PtBMA on cellulose membrane versus reaction time, ATRP: $1.5 \mathrm{~mol} / \mathrm{L}$ tBMA solution in MeCN / water solution mixture $(\mathrm{V}: \mathrm{V}=9: 1)$ with a catalyst system of $[t \mathrm{BMA}]:[\mathrm{CuCl}]:\left[\mathrm{CuCl}_{2}\right]:[\mathrm{Bpy}]=$ 75:1:0.1:2.75 at ambient temperature.

Figure 4.48 Mass of Al (open columns), PtBMA (black columns) and PMAA (gray columns) gafted cellulose membrane versus ATRP reaction time, ATRP in $1.5 \mathrm{~mol} / \mathrm{L}$ tBMA solution in MeCN / water solution mixture $(\mathrm{V}: \mathrm{V}=9: 1)$ with a catalyst system of $[t \mathrm{BMA}]:[\mathrm{CuCl}]:\left[\mathrm{CuCl}_{2}\right]:[\mathrm{Bpy}]=$ 75:1:0.1:2.75 at ambient temperature; hydrolysis of the PtBMA-g-cellulose membranes in 50\% trifluoroacetic acid solution in MeCN / water mixture $(\mathrm{V}: \mathrm{V}=1: 1)$ at $50^{\circ} \mathrm{C}$ for $14 \mathrm{~h}$.

Figure 4.49 Lost mass of base cellulose membrane $(\varnothing 18 \mathrm{~mm})$ versus TFA concentration in MeCN / water solution mixture $(\mathrm{V}: \mathrm{V}=1: 1)$ at $50^{\circ} \mathrm{C}$ for $14 \mathrm{~h}$.

Figure 4.50 СуC binding capacity of PMAA-g- cellulose membrane versus TFA concentration in $\mathrm{MeCN} /$ water solution mixture $(\mathrm{V}: \mathrm{V})=1: 1$ at $50^{\circ} \mathrm{C}$ for $14 \mathrm{~h}$. ATRP in $1.5 \mathrm{~mol} / \mathrm{L}$ tBMA solution in $\mathrm{MeCN} /$ water solution mixture $(\mathrm{V}: \mathrm{V}=9: 1)$ with a catalyst system of $[t \mathrm{BMA}]:[\mathrm{CuCl}]:\left[\mathrm{CuCl}_{2}\right]:[\mathrm{Bpy}]=75: 1: 0.1: 2.75$ at ambient temperature for $5 \mathrm{~h}$; hydrolysis of the PtBMA-g-cellulose membranes in trifluoroacetic acid solution in $\mathrm{MeCN} /$ water mixture $(\mathrm{V}: \mathrm{V}=1: 1)$ at $50{ }^{\circ} \mathrm{C}$ for $14 \mathrm{~h}$; CyC adsorption in $0.2 \mathrm{~g} / \mathrm{L}$ protein phosphate solution ( $\mathrm{pH}=7.0,5 \mathrm{mmol} / \mathrm{L}$ ) for $2 \mathrm{~h}$. 
Figure 4.51 Contact angle results for membranes after different stages of functionalization. Al corresponds to a membrane after ATRP initiator immobilization; PtBMA and PMAA correspond to the membranes grafted with PtBMA (5 h ATRP) and PMAA (35\% TFA, $14 \mathrm{~h}, 50^{\circ} \mathrm{C}$ ) respectively.

Figure 4.52 Degree of grafting for PAAm PMAA-g-cellulose membrane surface after ATRP $(5 \mathrm{~h})$ and hydrolysis versus the irradiation time: $0.3 \mathrm{~mol} / \mathrm{L}$ AAm and MBAA with degree of crosslinking 10\%, irradiation under the UV-intensity 24 $\mathrm{mW} / \mathrm{cm}^{2}$.

Figure 4.53 Influence of UV-irradiation time on the properties of Lyz-imprinted cellulose membranes: the Lyz and CyC binding capacities (open and solid columns) and resulting Lyz vs. CyC selectivity (squares) of the different MIP membranes with the same length of scaffold chains (ATRP $3 \mathrm{~h}$; UV: $0.3 \mathrm{~mol} / \mathrm{L}$ functional monomer; AAm:MBAA = 9:1, i.e. degree of crosslinking 10\%; UV-intensity about $24 \mathrm{~mW} / \mathrm{cm}^{2}$ ).

Figure 4.54 Influence of UV-monomer concentration on the properties of Lyz-imprinted cellulose membranes: the Lyz and CyC binding capacities (open and solid columns) and resulting Lyz vs. CyC selectivity (squares) of the different MIP membranes with the same length of scaffold chains (ATRP 3 h; UV: 0.3 and 0.5 mol/L functional monomer; AAm:MBAA = 9:1, i.e. degree of crosslinking 10\%; UV-intensity about $64 \mathrm{~mW} / \mathrm{cm}^{2} ; 12 \mathrm{~min}$ ).

Figure 4.55 Influence of UV-intensity concentration on the properties of Lyz-imprinted cellulose membranes: the Lyz and CyC binding capacities (open and solid columns) and resulting Lyz vs. CyC selectivity (squares) of the different MIP membranes with the same length of scaffold chains (ATRP 3 h; UV: 0.3 and 0.5 mol/L functional monomer; AAm:MBAA = 9:1, i.e. degree of crosslinking 10\%).

Figure 4.56 Influence of UV-irradiation time on the properties of Lys-imprinted cellulose membranes: the Lyz and CyC binding capacities (open and solid columns) and resulting Lyz vs. CyC selectivity (squares) of the different MIP membranes with the same length of scaffold chains (ATRP: $3 \mathrm{~h}$; UV: $0.5 \mathrm{~mol} / \mathrm{L}$ functional monomer; AAm:MBAA = 9:1, i.e. degree of crosslinking 10\%; UV-intensity about $71.3 \mathrm{~mW} / \mathrm{cm}^{2}$ ). 
Figure 4.57 Temperature versus irradiation time under the UV-intensity about 71.3 $\mathrm{mW} / \mathrm{cm}^{2}$ in UVA Print 100-200.

Figure 4.58 Influence of crosslinking degree on the properties of Lys-imprinted cellulose membranes: the Lyz and CyC binding capacities (open and solid columns) and resulting Lyz vs. CyC selectivity (squares) of the different MIP membranes with the same length of scaffold chains (ATRP: $3 \mathrm{~h}$; UV: $0.5 \mathrm{~mol} / \mathrm{L}$ functional monomer of AAm:MBAA; UV-intensity about $71.3 \mathrm{~mW} / \mathrm{cm}^{2}, 10 \mathrm{~min}$ ).

Figure 4.59 Influence of UV-irradiation time on the properties of Lys-imprinted cellulose membranes: the Lyz and CyC binding capacities (open and solid columns) and resulting Lyz vs. CyC selectivity (squares) of the different MIP membranes with the same length of scaffold chains (ATRP: $3 \mathrm{~h}$; UV: $0.5 \mathrm{~mol} / \mathrm{L}$ functional monomer of AAm:MBAA with crosslinking degree =15\%; UV-intensity about $71.3 \mathrm{~mW} / \mathrm{cm}^{2}$ ).

Figure 4.60 Influence of scaffold length on the properties of Lys-imprinted cellulose membranes: the Lyz and CyC binding capacities (open and solid columns) and resulting Lyz vs. CyC selectivity (squares) of the different MIP membranes with the same length of scaffold chains (ATRP: 1 to $3 \mathrm{~h}$; UV: $0.5 \mathrm{~mol} / \mathrm{L}$ functional monomer of AAm:MBAA with crosslinking degree = 15\%; UV-intensity about $\left.71.3 \mathrm{~mW} / \mathrm{cm}^{2}, 8 \mathrm{~min}\right)$.

Figure 4.61 Comparison of single protein adsoption and in situ separation of protein mixture.

Figure 4.62 UV-Vis spectrums of eluate from in situ experiment (black curve), and the pure Lyz (blue curve) and CyC (red curve) phosphate solutions with concentration $50 \mathrm{mg} / \mathrm{L}$.

Figure 4.63 Lyz (open columns) and CyC (solid columns) binding capacities and Lyz to CyC selectivities (squares) for MIP, NIP and scaffold membranes (ATRP: 1 to h; UV: $0.5 \mathrm{~mol} / \mathrm{L}$ functional monomer of $\mathrm{AAm}: \mathrm{MBAA}$ with crosslinking degree $=15 \%$; UV-intensity about $68 \mathrm{~mW} / \mathrm{cm}^{2}, 8 \mathrm{~min}$ ).

Figure 4.64 Lyz binding isotherms of MIP (solid squares) and NIP (solid circles) cellulose membranes as well as curves obtained by fitting the data to the Langmuir model. 
Fgirue 4.65 Degree of grafting for PtBMA on PET membrane surface with immobilized benzyl chloride (squares) versus the reaction time of ATRP in $1.5 \mathrm{~mol} / \mathrm{L} t \mathrm{BMA}$ solution in MeCN / water solution mixture ( $\mathrm{V}: \mathrm{V}=9: 1)$ with a catalyst system of $[t B M A]:[C u C l]:[$ PMDETA $]=75: 1: 1.25$ at ambient temperature .

Fgirue 4.66 IR spectra of benzychloride immobilized PET membrane and the PtBMA-g-PET membrane via $6 \mathrm{~h}$ ATRP.

Figure 4.67 Comparison of membrane mass after benzyl chloride immobilization (CBC, open columns) and after subsequent $48 \mathrm{~h}$ DTC immobilization (DTC, solid columns ), in different solvents.

Figure 4.68 Degree of grafting for PAAm on DTC immobilized membrane in $0.3 \mathrm{~mol} / \mathrm{L}$ AAm water solution, irradiation under the UV-intensity $24 \mathrm{~mW} / \mathrm{cm}^{2}$ for $20 \mathrm{~min}$.

Figure 4.69 Mechanism of phase transfer catalyst to accelerate the substitution reaction.

Figure 4.70 Mass increase versus the DTC immobilization time in 0.15 DTC mol/L containing $10 \% \operatorname{TBAB}(0.015 \mathrm{~mol} / \mathrm{L}) \mathrm{MeCN}$ solution.

Figure 4.71 Degree of grafting for PtBMA on PET membrane surface (squares) versus DTC immobilization time, ATRP: $1.5 \mathrm{~mol} / \mathrm{L}$ tBMA solution in MeCN /water solution mixture $(\mathrm{V}: \mathrm{V}=9: 1)$ with a catalyst system of $[$ tBMA $]:[\mathrm{CuCl}]:[\mathrm{PMDETA}]=75: 1$ : 1.25 at ambient temperature for $4 \mathrm{~h}$.

Figure 4.72 XPS spectra of the sulfur (2p) region of different membranes: black (PET): base TE PET membrane; red (CBC_PET): TE PET after benzyl chloride group immobilization; blue (DTC_PET): TE PET after DTC group immobilization for 24 h.

Figure 4.73 Degree of grafting for PAAm on PET membrane surface (squares) versus the UV-irradiation time; UV-polymerization: $0.7 \mathrm{~mol} / \mathrm{L} \mathrm{AAm} \mathrm{MeCN}$ solution, irradiation under the UV-intensity $24 \mathrm{~mW} / \mathrm{cm}^{2}$.

Figure 4.74 IR spectra of DTC immobilized PET membrane and the PAAm-g-PET membrane via $20 \mathrm{~min}$ UV-irradiation in $0.3 \mathrm{~mol} / \mathrm{L} \mathrm{AAm}$ water solution under the UV-intensity $24 \mathrm{~mW} / \mathrm{cm}^{2}$.

Figure 4.75 Contact angle results of membrane after different modification steps. Initiator benzyl chloride immobilization (CBC); initiator DTC immobilization; aATRP of PtBMA ;UV-irradiation of PAAm. 
Figure 4.76 Stability of DTC immobilized membrane in hydrolysis conditions at $50{ }^{\circ} \mathrm{C}$ and for $18 \mathrm{~h}$ with shaking $200 \mathrm{rpm}$ in different solutions, white: benzyl chloride immobilized membrane; black: DTC immobilized membrane; gray: DTC immobilized membrane after immersion in hydrolysis solution.

Figure 4.77 Stability of benzyl chloride and DTC immobilized membrane in water for $18 \mathrm{~h}$ at RT with shaking speed $200 \mathrm{rpm}$.

Figure 4.78 ATRP and UV-reactivity of benzyl chloride- and DTC immobilized membrane. ATRP: $1.5 \mathrm{~mol} / \mathrm{L}$ tBMA solution in solution mixture with a catalyst system of [tBMA]:[CUCl]:[PMDETA] = 75:1: 1.25 at ambient temperature for $4 \mathrm{~h}$; UV: 0.7 $\mathrm{mol} / \mathrm{L}$ AAm MeCN solution, irradiation under the UV-intensity $62 \mathrm{~mW} / \mathrm{cm}^{2}$ for $10 \mathrm{~min}$.

Figure 4.79 DG of PAAm (red points) via UV-initiation and DG of PtBMA (black squares) via ATRP, both versus DTC immobilization time with initiation sequence UVinitiation $\rightarrow$ ATRP; UV: UV: $0.7 \mathrm{~mol} / \mathrm{L}$ AAm MeCN solution, irradiation under the UV-intensity $62 \mathrm{~mW} / \mathrm{cm}^{2}$ for $10 \mathrm{~min}$; ATRP: $1.5 \mathrm{M}$ tBMA, catalyst system $[$ tBMA]:[CuCl]:[PMDETA] = 75:1:1.25 in MeCN for $4 \mathrm{~h}$.

Figure 4.80 DG of PAAm (red points) via UV-initiation and DG of PtBMA (black squares) via ATRP versus DTC immobilization time with initiation sequence ATRP $\rightarrow$ UVinitiation; UV: UV: $0.7 \mathrm{~mol} / \mathrm{L}$ AAm MeCN solution, irradiation under the UVintensity $62 \mathrm{~mW} / \mathrm{cm}^{2}$ for $15 \mathrm{~min}$; ATRP: $1.5 \mathrm{M}$ tBMA, catalyst system $[$ tBMA]:[CuCl]:[PMDETA] = 75:1:1.25 in MeCN for $4 \mathrm{~h}$.

Figure 4.81 Complex of DTC and copper formed on the membrane surface.

Figure 5.1 Summary of Lyz- and IgG-imprinting. Squares represent the Lyz imprinting and points represent the IgG imprinting, legends represent the imprinting condition in form of DG [min]/crosslinking degree/UV-irradiation time [min], the same color means the same series.

Figure 5.2 Comparison of Lyz imprinting on TE PET membrane (squares) and cellulose membrane (points) using selectivity from single protein adsorption; legends represent the imprinting condition in form of DG [min]/crosslinking degree/UV-irradiation time [min], the same color means the same series. 


\section{Appendix}

\section{Curriculum Vita}

\section{PERSONAL INFORMATIOM}

Name: Dongxu Yin

Place of Birth: Anhui, P. R. China

Birth date: 12/1983

\section{EDUCATION}

09.2001-07.2005

B. Sc. in Chemical Engineering: Major in Polymer Materials and Engineering

Beijing Institute of Petrochemical Technology, Beijing, China

12.2005-09.2006 German Language Course and Graduation with DSH-2

University Duisburg-Essen, Essen, Germany

10.2006-12.2009 M. Sc. in Chemistry: Major in Polymer Synthesis

University Duisburg-Essen, Essen, Germany

Since $01.2010 \quad$ Ph.D. Student in Chemistry, Major in Protein Imprinting

Ph.D. thesis: Protein-selective Adsorbers by Molecular Imprinting via Novel Two-step Surface Grafting Method

Supervisor: Prof. Mathias Ulbricht

University Duisburg-Essen, Essen, Germany 


\section{Conferences}

1. "Stand der Membrantechnik im industriellen Einsatz", March 23, 2010, Frankfurt, Germany.

2. “Downstream Days" Summer School 2010, Sep. 29.-Oct. 01, 2010, Wetter, Germany

3. D. Yin, M. Ulbricht, "Protein-selective adsorber by molecular imprinting via two-step surface grafting", Frontiers in Polymer Science, May 29-31, 2011, Lyon, France (poster)

4. D. Yin, M. Ulbricht, "Protein-selective adsorber by molecular imprinting via two-step surface grafting", 4th Graduate Student Symposium on Molecular Imprinting, September 28-30, 2011, London, United Kingdom (oral presentation)

5. D. Yin, M. Ulbricht, "Protein-selective adsorber by molecular imprinting via two-step surface grafting", 7th International Conference on Molecular Imprinting-MIP2012, August 27-30, 2012, Paris, France (poster)

\section{Publications}

1. D. Yin, M. Ulbricht, "Protein-selective adsorbers by molecular imprinting via two-step surface grafting", Journal of Materials Chemistry B, 2013, 1, 3209-3219

2. D. Yin, M. Ulbricht, "Lysozyme imprinted cellulose membrane", in preparation

3. D. Yin, M. Ulbricht, "Antibody-imprinted membranes via via two-step surface grafting”, in preparation

4. D. Yin, M. Ulbricht, "Novel orthogonal initiation of graft copolymerizations by combining surface initiated ATRP and RAFT", in preparation 